\title{
THE VALUATION OF PRODUCT INTRODUCTION TIME STRATEGIES USING A REAL OPTIONS APPROACH
}

\author{
by \\ Mehdi Hossein Yazdi \\ M.Sc., Iran University of Science and Technology, Tehran, Iran, 2005
}

\author{
A dissertation \\ presented to Ryerson University \\ in partial fulfillment of the \\ requirements for the degree of \\ Doctor of Philosophy \\ in the program of \\ Mechanical and Industrial Engineering
}

Toronto, Ontario, Canada, 2019

(C)Mehdi Hossein Yazdi 2019 


\section{AUTHOR'S DECLARATION FOR ELECTRONIC SUBMISSION OF A DISSERTATION}

I hereby declare that I am the sole author of this dissertation. This is a true copy of the dissertation, including any required final revisions, as accepted by my examiners.

I authorize Ryerson University to lend this dissertation to other institutions or individuals for the purpose of scholarly research.

I further authorize Ryerson University to reproduce this dissertation by photocopying or by other means, in total or in part, at the request of other institutions or individuals for the purpose of scholarly research.

I understand that my dissertation may be made electronically available to the public. 
The Valuation of Product Introduction Time

Strategies Using a Real Options Approach

Doctor of Philosophy 2019

Mehdi Hossein Yazdi

Mechanical and Industrial Engineering

Ryerson University

\begin{abstract}
In today's economy, new technologies rapidly emerge in the durable goods market. Therefore, it is paramount for manufacturing companies to optimize product introduction time for their second-generation product (SGP), which is an upcoming product in the production line, when profitability is at stake. The main factors affecting this timing are technology advancements and changes in customer tastes, which make determining an optimal introduction time for a product a challenging task. The main goal of this research is to find the optimal product introduction time for the SGP that maximizes the net present value (NPV) over a given period while product life cycle (PLC), pricing, and advertising are explicitly being taken into consideration.

Demand for each product contains two regimes life cycle, and each regime is defined by a geometric Brownian motion (GBM). Each GBM has a drift rate and volatility. Moreover, there is a correlation between different regimes for different products. Correlated GBMs are discretized using a lattice approach. The Bass model is used to determine demand parameters including drift, volatility, and correlation, while dynamic programming is used to optimize NPV. Flexibilities, such as expansion, contraction, and switching, are identified between two products. Examples are provided to show the applicability of the developed models. Accordingly, the results show as the drift rate, volatility, and initial demand for a first-generation product (FGP) increase, the SGP introduction time has to be delayed. Furthermore, results demonstrate that in decreasing pricing policy, the SGP has to be introduced as early as the FGP. In increasing-decreasing pricing policy, as the increasing pricing rate is increasing, the SGP introduction time is delayed. When the advertising budget percentage increases, the
\end{abstract}


NPV increases up to a certain level and then it will be saturated. Major contributions of this thesis are as follows: first, investigating the product introduction time by integrating marketing and manufacturing aspects; second, developing a model to incorporate the flexibility and production cost of the system for determining the optimal product introduction time. Third, the value of the product introduction time is expressed in terms of dollar value and this would help managers to make decisions easily. The models developed in this research can be used as practical tools for manufacturers to find the optimal product introduction time (PIT) and also the research can be used as a guideline to introduce the second-generation PIT. 


\section{Acknowledgements}

I am grateful to my supervisor, Dr. M. Wahab M. Ismail, Department of Mechanical and Industrial Engineering, Ryerson University, Toronto, Canada. He has unique excellent qualities. His critical thinking in doing research and supervision and his guidance in achieving a goal are sources of valuable experience and fine memories for me. I have learned a lot from him.

I am thankful to the committee members, Dr. Ali Ulku, Dr. Saeed Zolfaghari, Dr. Liping Fang, Dr. Bo Tan, and Dr. Ahmad Ghasempoor for their valuable comments and suggestions that improved the exposition of this dissertation. Thanks to my friend Dr. Payman Ahi for his friendly help and encouragement. I also thank all other faculty and staff members of the Department of Mechanical and Industrial Engineering, Ryerson University, Toronto, Canada for their cordial help and support.

My debt to my parents is forever unpayable. No words of acknowledgement will suffice to acknowledge their selfless sacrifices for me. I express my gratitude to my wife and son for enduring the 'entropy' of $\mathrm{PhD}$ research from time to time. 


\section{Table of Contents}

$\begin{array}{ll}\text { Abstract } & \text { iii }\end{array}$

List of Tables $\quad$ ix

List of Figures $-x$

Nomenclature $\quad$ xiv

1 Introduction $\quad 1$

1.1 Research motivation and new product introduction time . . . . . . . . . 1

1.2 Research objectives . . . . . . . . . . . . . . . . . 5

1.3 Research contributions ..................... 5

1.4 Organization of the thesis . . . . . . . . . . . . . 6

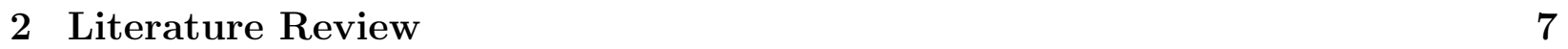

2.1 Timing ............................. 7

2.2 Pricing . . . . . . . . . . . . . . . . . . . . . . 10

2.3 Advertising . . . . . . . . . . . . . . . . . . 13

2.4 Real options . . . . . . . . . . . . . . . . . . . . . . . 15

2.5 Gaps in the literature . . . . . . . . . . . . . . . . 16

3 Methodology $\quad 18$

3.1 Problem statement . . . . . . . . . . . . . . . . . 18

3.2 Overall methodology . . . . . . . . . . . . . . . . . . . . 21

3.2.1 Estimating $p, q$, and $m \ldots \ldots \ldots \ldots$

3.3 Value options methods . . . . . . . . . . . . . . . . . . . . 29 
4.1 Introduction . . . . . . . . . . . . . . . . . 34

4.2 Model development . . . . . . . . . . . . . . . . . . . . . 35

4.3 Estimating the stochastic demand parameters . . . . . . . . . . . 36

4.4 Lattice approach . . . . . . . . . . . . . . . . . . . . 39

4.5 Cost function . . . . . . . . . . . . . . . . . . . 44

4.6 Flexible systems and valuating the sequential product introduction strategy . 46

4.7 Research Analysis Method . . . . . . . . . . . . . . . . . . . 48

4.7 .1 Initial demand and PLC . . . . . . . . . . . . . . . 49

4.7.2 Effect of the profit margin on the second-generation PIT . . . . . . 55

4.7.3 Effect of the demand uncertainty on the second-generation PIT . . . 58

4.8 Summary . . . . . . . . . . . . . . . . . . . 62

5 The Valuation of Product Introduction Strategies Considering PLC and $\begin{array}{ll}\text { Pricing } & 63\end{array}$

5.1 Introduction . . . . . . . . . . . . . . . . . 63

5.2 Model development . . . . . . . . . . . . . . . . . . . 65

5.3 Estimating the stochastic demand parameters . . . . . . . . . . 65

5.4 Lattice approach . . . . . . . . . . . . . . . . . . . 69

5.4.1 When price changes the drift rate . . . . . . . . . . . 70

5.4 .2 When price changes both volatility and drift rate . . . . . . . 73

5.5 Analysis and results . . . . . . . . . . . . . . . . . 76

5.5.1 Total replacement . . . . . . . . . . . . . . 77

5.5.2 Phase out transition .................... 80

5.5.3 Phase out transition in increasing-decreasing pricing policy when decreasing pricing rate is changed . . . . . . . . . . . . . . 83

5.5.4 Phase out transition in increasing-decreasing pricing policy when increasing pricing rate is changed . . . . . . . . . . . 86

5.6 Summary . . . . . . . . . . . . . . . . . . . . 89

6 The Valuation of Product Introduction Strategies Considering PLC, Pric$\begin{array}{ll}\text { ing and Advertising } & 90\end{array}$ 
6.1 Introduction . . . . . . . . . . . . . . . . . . . . . 9 90

6.2 Model development . . . . . . . . . . . . . . . . . . . . . . . . 92

6.3 Estimating the stochastic demand parameters . . . . . . . . . . . . . 92

6.4 Analysis and results . . . . . . . . . . . . . . . . . . . . . . 97

6.4 .1 Total replacement . . . . . . . . . . . . . . . . . . . . . . . . . . . . 98

6.4 .2 Phase out transition . . . . . . . . . . . . . . . . . . . . . . . . 101

6.4.3 Phase out transition in increasing-decreasing pricing policy when decreasing pricing rate is changed . . . . . . . . . . . . . 104

6.4.4 Phase out transition in increasing-decreasing pricing policy when increasing pricing rate is changed $\ldots \ldots \ldots \ldots$. . . . . . 107

6.5 Cross functional advertising . . . . . . . . . . . . . . . . . . . 110

6.6 Comparing the results of Chapter 5 and Chapter $6 \ldots \ldots 114$

6.7 Summary . . . . . . . . . . . . . . . . . . . . . 116

$\begin{array}{lll}7 & \text { Conclusion and Future work } & 117\end{array}$

7.1 Research contributions . . . . . . . . . . . . . . . . . . . . . 120

7.2 Challenges, limitations and future work . . . . . . . . . . . . . 121

$\begin{array}{lr}\text { Appendices } & 123\end{array}$

$\begin{array}{lll}\text { Appendix A When price changes the drift rate } & 123\end{array}$

$\begin{array}{lll}\text { Appendix B When price changes both volatility and drift rate } & 127\end{array}$

Appendix C Step size and Branch Probabilities by Matching the First and Second Moments

$\begin{array}{ll}\text { Appendix D Uncorrelating the correlated processes } & 133\end{array}$

$\begin{array}{lr}\text { References } & 135\end{array}$ 


\section{List of Tables}

1.1 Apple iPhone generations introduction time . . . . . . . . . . . . 2

3.1 Sales data (in thousands) from IBM for generations 3 and 4 of main frame

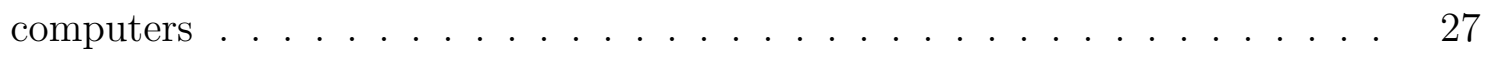

3.2 Estimated Bass parameters for products $A$ and $B \ldots \ldots . . . . . . . .27$

4.1 Estimated Bass parameters for products $A$ and $B \ldots . . . . . . . .36$

4.2 Estimated demand parameters for product $A \ldots \ldots$. . . . . . . . 39

4.3 Estimated demand parameters for product $B$. . . . . . . . . . . . 39

4.4 Estimated correlated demand for combined regimes . . . . . . . . . . . . 39

4.5 Instantaneous mean and volatility of uncorrelated processes . . . . . . . . . . 42

5.1 Estimated Bass parameters for products $A$ and $B \ldots \ldots 6$

5.2 Estimated demand parameters for product $A \ldots \ldots$. . . . . . . 69

5.3 Estimated demand parameters for product $B$. . . . . . . . . . . . . 69

5.4 Estimated correlated demand for combined regimes . . . . . . . . . . . . 69

5.5 Instantaneous mean and volatility of uncorrelated processes . . . . . . . . . 73

5.6 Instantaneous mean and volatility of uncorrelated processes . . . . . . . . 76

6.1 Estimated Bass parameters for products $A$ and $B \ldots \ldots 3$

6.2 Estimated demand parameters for product $A \ldots \ldots$. . . . . . . 96

6.3 Estimated demand parameters for product $B$. . . . . . . . . . . . . . 97

6.4 Estimated correlated demand for combined regimes . . . . . . . . . . . . 97 


\section{List of Figures}

1.1 Product life cycle $\ldots \ldots \ldots \ldots$

3.1 Demand profile for products $A$ and $B \ldots \ldots \ldots \ldots$

3.2 Overall framework of methodology f . . . . . . . . . . . . . . . . 22

3.3 Total replacement-sequential strategy . . . . . . . . . . . . . . . . . . 23

3.4 Phase out transition-sequential strategy $\ldots \ldots \ldots \ldots$

3.5 Phase out transition-simultaneous strategy . . . . . . . . . . . . . . . . . 24

3.6 A pentanomial lattice demand value . . . . . . . . . . . . . . . . 31

3.7 Corresponding profit in a pentanomial lattice . . . . . . . . . . . . . . . 32

3.8 Expected profit corresponding to Figure 3.7 in a pentanomial lattice . . . . . 33

4.1 Overall framework of methodology $\ldots \ldots \ldots$

4.2 Total and marginal production cost . . . . . . . . . . . . . . 46

4.3 Second-generation product introduction time for different drift rates of product $A$ in growth regime-Scenario $1 \ldots \ldots \ldots$

4.4 Second-generation product introduction time for different drift rates of product $A$ in growth regime-Scenario $1 \ldots \ldots \ldots$

4.5 Second-generation PIT for different initial demands of product $A$-Scenario 151

4.6 Second-generation PIT for different initial demands of product $A$-Scenario $1 \quad 52$

4.7 Second-generation PIT for different drift rates of product $A$ in growth regime 53

4.8 Second-generation PIT for different drift rates of product $A$ in growth regime 53

4.9 Second-generation PIT for different initial demand of product $A \ldots \ldots 54$

4.10 Second-generation PIT for different initial demand of product $A \ldots \ldots 55$

4.11 Second-generation PIT for different unit costs of product $A \ldots \ldots$. . . . 56

4.12 Second-generation PIT for different unit costs of product $A \ldots \ldots 56$ 
4.13 Second-generation PIT for different unit costs of product $A \ldots$. . . . . 57

4.14 Second-generation PIT for different unit costs of product $A$. . . . . . 58

4.15 Second-generation PIT for different volatilities of product $A$ in growth regime 59

4.16 Second-generation PIT for different volatilities of product $A$ in growth regime 59

4.17 Second-generation PIT for different volatilities of product $A$ in growth regime 60

4.18 Second-generation PIT for different volatilities of product $A$ in growth regime 61

5.1 Overall framework of methodology . . . . . . . . . . . . . . . . 64

5.2 Second-generation PIT (Decreasing pricing policy-total replacement) . . . 77

5.3 Second-generation PIT (Decreasing pricing policy-total replacement) . . . . 78

5.4 Second-generation PIT (Increasing-Decreasing pricing policy-total replacement) 79

5.5 Second-generation PIT (Increasing-Decreasing pricing policy-total replacement) 79

5.6 Second-generation PIT with Approach (I) (Decreasing pricing policy-phase out transition) . . . . . . . . . . . . . . . . 80

5.7 Second-generation PIT with Approach (I) (Decreasing pricing policy-phase out transition) . . . . . . . . . . . . . . . . . . 81

5.8 Second-generation PIT with Approach (II) (Decreasing pricing policy-phase

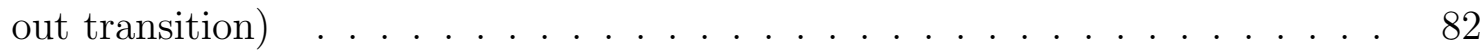

5.9 Second-generation PIT with Approach (II) (Decreasing pricing policy-phase out transition) . . . . . . . . . . . . . . . . . 82

5.10 Second-generation PIT with Approach (I) (Increasing-Decreasing pricing policyphase out transition) . . . . . . . . . . . . . . . . . 83

5.11 Second-generation PIT with Approach (I) (Increasing-Decreasing pricing policyphase out transition) . . . . . . . . . . . . . . . . . . 84

5.12 Second-generation PIT with Approach (II) (Increasing-Decreasing pricing policyphase out transition) . . . . . . . . . . . . . . . . . . 85

5.13 Second-generation PIT with Approach (II) (Increasing-Decreasing pricing policyphase out transition) . . . . . . . . . . . . . . . 85

5.14 Second-generation PIT with Approach (I) (Increasing-Decreasing pricing policyphase out transition) . . . . . . . . . . . . . . . . . . . 86 
5.15 Second-generation PIT with Approach (I) (Increasing-Decreasing pricing policyphase out transition) . . . . . . . . . . . . . . . . 87

5.16 Second-generation PIT with Approach (II) (Increasing-Decreasing pricing policyphase out transition) . . . . . . . . . . . . . . . . . . . . . 88

5.17 Second-generation PIT with Approach (II) (Increasing-Decreasing pricing policyphase out transition) . . . . . . . . . . . . . . . . . . 88

6.1 Overall framework of methodology . . . . . . . . . . . . . . . . . . . . 91

6.2 Second-generation product introduction time (Decreasing pricing policy-total replacement) . . . . . . . . . . . . . . . . . . . . . . . 98

6.3 Second-generation product introduction time (Decreasing pricing policy-total replacement) . . . . . . . . . . . . . . . . . . . 99

6.4 Second-generation product introduction time (Increasing-Decreasing pricing policy-total replacement) . . . . . . . . . . . . . . . . . . . 100

6.5 Second-generation product introduction time (Increasing-Decreasing pricing policy-total replacement) . . . . . . . . . . . . . . . . . . 100

6.6 Second-generation product introduction time with Approach (I) (Decreasing pricing policy-phase out transition) . . . . . . . . . . . . . . . . . 101

6.7 Second-generation product introduction time with Approach (I) (Decreasing pricing policy-phase out transition) . . . . . . . . . . . . . . . . 102

6.8 Second-generation product introduction time with Approach (II) (Decreasing pricing policy-phase out transition) . . . . . . . . . . . . . . 103

6.9 Second-generation product introduction time with Approach (II) (Decreasing pricing policy-phase out transition) . . . . . . . . . . . . . . 103

6.10 Second-generation product introduction time with Approach (I) (IncreasingDecreasing pricing policy-phase out transition) . . . . . . . . . . . . . . . 104

6.11 Second-generation product introduction time with Approach (I) (IncreasingDecreasing pricing policy-phase out transition) . . . . . . . . . . . 105

6.12 Second-generation product introduction time with Approach (II) (IncreasingDecreasing pricing policy-phase out transition) . . . . . . . . . . . 106 
6.13 Second-generation product introduction time with Approach (II) (IncreasingDecreasing pricing policy-phase out transition) . . . . . . . . . . . . 106

6.14 Second-generation product introduction time with Approach (I) (IncreasingDecreasing pricing policy-phase out transition) . . . . . . . . . . . . 107

6.15 Second-generation product introduction time with Approach (I) (IncreasingDecreasing pricing policy-phase out transition) . . . . . . . . . . . . 108

6.16 Second-generation product introduction time with Approach (II) (IncreasingDecreasing pricing policy-phase out transition) . . . . . . . . . . . . . 109

6.17 Second-generation product introduction time with Approach (II) (IncreasingDecreasing pricing policy-phase out transition) . . . . . . . . . . . . . 109

6.18 Second-generation product introduction time when advertising budget percentage changes (Decreasing pricing policy-phase out transition) . . . . . . . 111

6.19 Second-generation product introduction time when advertising budget percentage changes (Decreasing pricing policy-phase out transition) . . . . . . . 111

6.20 Second-generation product introduction time when advertising budget percentage changes (Increasing-Decreasing pricing policy-phase out transition)

6.21 Second-generation product introduction time when advertising budget percentage changes (Increasing-Decreasing pricing policy-phase out transition) . 113

6.22 Advertising budget percentage changes (Decreasing pricing policy-phase out transition)

6.23 Advertising budget percentage changes (Increasing-Decreasing pricing policyphase out transition) . . . . . . . . . . . . . . . . . . 114 


\section{Nomenclature}

\begin{tabular}{|c|c|}
\hline$p$ & Innovation coefficient, external influence \\
\hline$\tau$ & Delay time \\
\hline$m$ & Market size \\
\hline$t_{A}$ & Switching time for product $\mathrm{A}$ \\
\hline$t_{B}$ & Switching time for product B \\
\hline$\mu$ & Drift rate of the product \\
\hline$\rho$ & Correlation between two generation of the product \\
\hline$\sigma$ & Volatility of the product \\
\hline$\theta$ & Demand of the product \\
\hline$\phi$ & Demand step size \\
\hline$W$ & Wiener process \\
\hline$C N D$ & Cumulative normal distribution \\
\hline$G B M$ & Geometric Brownian motion \\
\hline$N P V$ & Net present value \\
\hline$P D F$ & Probability distribution function \\
\hline$P L C$ & Product life cycle \\
\hline$q$ & Imitation coefficient, word of mouth effect \\
\hline
\end{tabular}




$\begin{array}{ll}\text { RSP } & \text { Regime switching probability } \\ \text { FGP } & \text { Product introduction time } \\ \text { SGP } & \text { Second-generation product } \\ \omega & \text { Group of uncorrelated processes } \\ R_{j} & \text { Rank of the uncorrelated process } j \\ d & \text { Decay regime } \\ g & \text { Growth regime } \\ j & \text { Uncorrelated process }\end{array}$




\section{Chapter 1}

\section{Introduction}

\subsection{Research motivation and new product introduction time}

Many studies have attempted to find the optimal time for launching new products into the market. A delay in the introduction of a new product can decrease the market value of the firm offering it by an average of 5.25\% (Hendricks and Singhal, 1997). In addition, a delay can lead to long-term loss of market share. For example, Hewlett-Packard lost $\$ 200$ million due to a six-month delay in shipping the HP300 series 930 computers (Waldman, 1986). IBM lost an estimated $\$ 100$ million in revenues out of total projected revenues of $\$ 700$ million (Markoff, 1991). Similarly, a one-week delay in the introduction of the Cray-3 supercomputer by Cray Computer Corporation led to $\$ 30$ million worth of order cancellations (Fisher, 1991), while a six-month delay in Microsoft's Window's NT development costed it $\$ 16$ million (Zachary, 1993). Hence, determining the optimum product introduction time is important for companies to maximize NPV.

Higher competition in the global market, complicated customer needs, and a shorter product life cycle (PLC) have led to new requirements for designing new products. In light of this, researchers have attempted to find the optimal time for launching new products into the market. Developing new products is a strategic process conducted in every business. New products lead to more profit, which is vital for a firm to stay in the market. Even monopolist firms rely on introducing a totally new product or redesigning an existing product 
or service because other firms are continually introducing or improving their products or services according to the feedback received from the market (Jiang and Jain, 2012a). Apple, for example, a company that produces a specific product, is constantly improving and introducing new products. Apple is one of the leading companies in innovation, unique design, and creativity. It took Apple five years to create the original iPhone, according to Apple's chief design officer Jony Ive. The Apple New Product Process (ANNP) is a document that describes in detail each step for developing the Apple products (O'Rourke, 2012). Table 1.1 shows the introduction time of each generation of the Apple iPhone.

Table 1.1: Apple iPhone generations introduction time

\begin{tabular}{|l|l||l|l|}
\hline Generation & $\begin{array}{l}\text { Introduction } \\
\text { Time }\end{array}$ & Generation & $\begin{array}{l}\text { Introduction } \\
\text { Time }\end{array}$ \\
\hline Original iPhone & Jan 9, 2007 & iPhone 6 Plus & Sep 19, 2014 \\
iPhone 3G & June 9, 2008 & iPhone 6S & Sep 9, 2015 \\
iPhone 3GS & June 8, 2009 & iPhone 6S Plus & Sep 9, 2015 \\
iPhone 4 & June 7, 2010 & iPhone SE & March 21, 2016 \\
iPhone 4S & Oct 4, 2011 & iPhone 7 & Sep 7, 2016 \\
iPhone 5 & Sep 12, 2012 & iPhone 7 Plus & Sep 7, 2016 \\
iPhone 5C & Sep 20,2013 & iPhone 8 & Sep 12, 2017 \\
iPhone 5S & Sep 20,2013 & iPhone 8 Plus & Sep 22, 2017 \\
iPhone 6 & Sep 19,2014 & iPhone X & Nov 3, 2017 \\
\hline
\end{tabular}

When launching a new product, the manager in charge is concerned about the optimal product introduction time. Different factors must be taken into account such as PLC, pricing, and advertising. PLC is a vitally important concept in marketing (Golder and Tellis, 2004; Qin and Nembhard, 2012). It represents the unit sales curve for some products, from the time it is introduced to the market until it is removed from the market (Rink and Swan, 1979). For every durable product, the stages in the PLC include introduction, growth, maturity, and decay. A general PLC is shown in Figure 1.1. Drift rate (increasing or decreasing rate of demand), volatility (uncertainty around the drift), and correlation (demand correla- 
tion between previous and current generations of the product) are the parameters that have to be considered for determining the best launching time. All firms and beneficiaries need to introduce a new version of a product or a service, and the introduction time is considered a strategic stage in the PLC (Golder and Tellis, 2004).

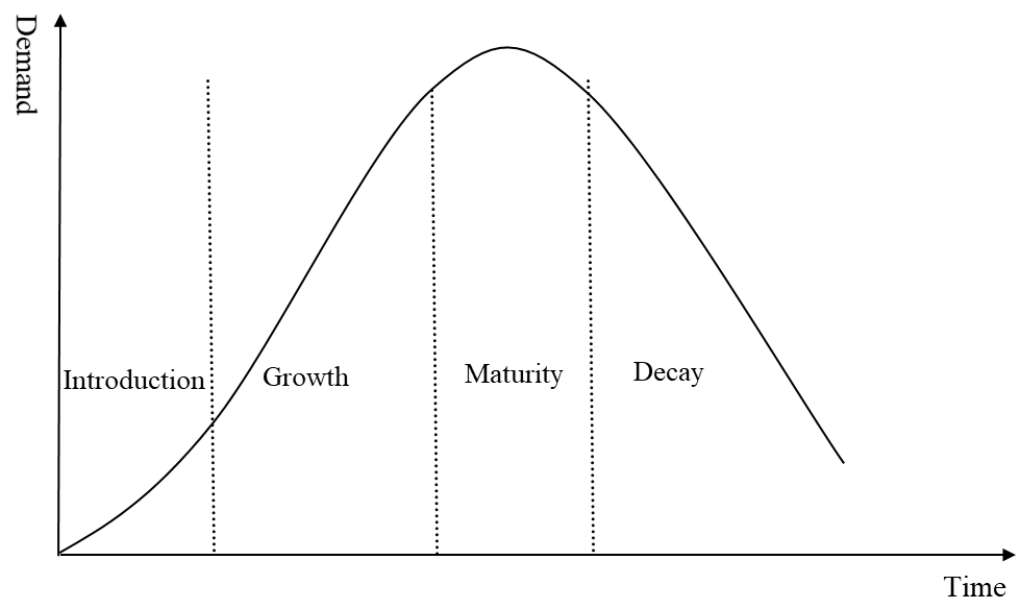

Figure 1.1: Product life cycle

Prices decline substantially over the PLC, especially during the early stages (Golder and Tellis, 2004). Pricing effect on the product demand parameters is a challenge for many companies. Pricing a new product affects the sales of both the previous generation and the current generation. Market skimming and market penetration are two pricing strategies that are discussed in the literature. Market skimming uses a high price initially and is good for developing markets. On the other hand, market penetration uses a low price initially to capture a large market share (Mahajan and Muller, 1991). Decreasing and increasing-decreasing pricing policy are two pricing policies that have been considered in this study. Bass et al. (2005) developed a model that could capture the effect of changes in price on demand. The generalized Norton Bass model (Jiang and Jain, 2012a) is used to capture the effect of the pricing and advertising on demand. Therefore, finding an optimal pricing strategy, especially for an upcoming product, is vital to maximize the profit over the PLC.

The advertising effect is another issue that has to be considered in product introduction time strategies. Advertising changes the price-value of the product by providing a new and compelling reason for the consumer to buy it. Advertising has a positive influence on the 
potential adopters which in turn has a positive impact on PLC. Percentage of sales is one of the most used approaches for determining advertising expenditures (Niu, 2006; Miller and Pazgal, 2007). As demand changes over time, the advertising policies vary too. Moreover, different advertising policies will affect sales. Accordingly, incorporating these parameters is a crucial task to help decision makers in the company find the optimum product introduction time. It is necessary for companies to consider all the involved parameters at the launch time of the new product.

Simultaneous and sequential strategies are two different strategies that have been considered in this study. When cannibalization is a problem, it is better to use the sequential strategy (Moorthy and Png, 1992). Cannibalization refers to a decrease in sales or market share of a product due to introducing a new generation product. Real options are used to evaluate the product introduction time. Real options are defined as a right, not an obligation, to expand, contract, or switch from one option to another. The real options approach is the most popular method for valuing an option and making the best decision in the future based on the different scenarios. As the demand is uncertain over time, real options enable valuating flexibilities. In other words, real options address the uncertainty. Employing financial and real options are considered the most common approaches in decision-making (Babich, 2006; Kamrad and Ord, 2006; Xu and Birge, 2006; Ding et al., 2007). For measuring the market price of risk, the capital asset pricing model is used, which enables managers to estimate the rate of return. In light of this, there is a dearth of practical tools for determining the optimal introduction time (Soleimani et al., 2012).

In many studies, lattice is used for a real options approach to assess the value of a real asset (Xiong and Zhang, 2016). Lattice is a numerical method and it is used for a discrete time model. The lattice approach is described in more detail in Section 3.3. This research follows the lattice method for evaluating the options introduced by Wahab (2006).

Dynamic programming is a recursive optimization method to solve complex problems by dividing them into a sequence of manageable smaller problems. The method is recursive, where a small part is defined and then an optimum solution is determined (Bertsekas, 2015). 


\subsection{Research objectives}

The main focus of this research is to develop a methodology that supports a decision-making tool for introducing sequential products, to help managers in charge, and to find the optimum product introduction time (PIT). The main goal of this research is to investigate the optimum PIT for second-generation products incorporating PLC, pricing, and advertising effects. The research is divided into three phases as follows:

- Phase I aims to find the optimum PIT for the second-generation product by maximizing the Net Present Value (NPV) over a given period of time with consideration of PLC.

- Phase II aims to find the optimum PIT for the second-generation product for different pricing policies by maximizing NPV over a given period of time with consideration of PLC and pricing effects.

- Phase III aims to find the optimum PIT for the second-generation product for a generic advertising policy within a certain level of advertising budget by maximizing NPV over a given period of time with consideration of PLC, pricing, and advertising effects.

\subsection{Research contributions}

This research makes several contributions to the literature and practice, namely:

- Maximizing the NPV by integrating marketing and manufacturing systems.

- Developing a methodology that determines the optimal sequential introduction time while incorporating the pricing and advertising effect.

Developing a model to incorporate the flexibility of the system for determining the optimal PIT.

- Introducing a coherent decision-making framework for sequential products in current research, which will maximize NPV over a given period of time by finding the optimal launching time for the second-generation product, considering the price policy for the FGP and the SGP. The pricing and advertising policy were considered for both first and SGPs. 
- Incorporating the production cost as a major parameter to find the optimum PIT in a manufacturing system.

- Developing a framework in the research to provide a strong basis for systematic integration of the involved marketing mix variables: timing, pricing, and advertising. This allows the investors to make decisions based on the estimated parameters and thereby find the optimum launch time for the SGP.

\subsection{Organization of the thesis}

The rest of the research is organized as follows: Chapter 2 provides the existing literature review and identifies the current research gaps. Chapter 3 presents research methodology. Developed models that address the thesis objectives are fully described in Chapters 4, 5, and

6. Conclusions and future work will be provided in Chapter 7, which includes summaries of contributions, limitations, and future work recommendations. 


\section{Chapter 2}

\section{Literature Review}

The focus of this chapter is to review the related literature in product entry timing, pricing, and advertising and to highlight where the major gaps exist. More specifically, the review is focused on the effect parameters of the SGP on the first-generation product parameters and on finding the optimal entry time for the SGP.

\section{$2.1 \quad$ Timing}

Determining the optimal product entry time into the market can be the most challenging issue for many companies. As new technologies emerge, new generations of products replace the older ones. These new generations are either a complete redesign or merely an improved version of their predecessors (Jiang and Jain, 2012b). In competitive markets, it is inevitable for companies to come up with new products in order to increase their market share. Thus, the introduction time for new product or service to the market is a critical decision.

Several studies have investigated optimal entry time into the market. These studies have analyzed either one-time sales or continuous service. One stream of research was proposed by Bass (1969), which described a diffusion model for timing the initial purchase of the new product. Bass's single product model reached the conclusion that there is an exponential growth from initial purchase to a peak, and then an exponential decay. Bass investigated the purchase of products by two groups: innovators and imitators. Innovators are people 
whose decision to adopt a product is independent of the decision of other individuals, and imitators are influenced by innovators over time. In Bass, the number of consumers that purchase a product is related to the number of previous buyers. Bass (1969) is the most tested and extended model, but this model did not differentiate between those who have already adopted an earlier generation of the product, and those who have not. In other words, Bass (1969) did not consider leapfrogging. Leapfrogging is defined as a situation in which potential adopters adopt a new generation of a product directly by skipping the previous version(s). Mahajan and Muller (1991) introduced the adoption model in the monopoly market for one unit purchase and developed the Bass diffusion model to investigate the replacement of the durable product for each successive generation. Mahajan and Muller (1991) predicted sales, determined the optimal entry time of new generations, and introduced a "now or at maturity" policy.

Numerous product introduction strategies have been proposed, involving factors such as the substitution and diffusion parameters when introducing a SGP. Norton and Bass (1987) developed a model for both substitution and diffusion. In the diffusion model, the first- and SGPs are allowed to run concurrently in the market, whereas in the substitution model, the first-generation product is replaced by second generation product. Switching from the first generation to the second generation is assumed in the paper written by Norton and Bass (1987). Norton and Bass (1987) studied the dynamic sale behavior of two product generations in a high technology industry. Norton (1989) developed a model to maximize the total profit for both the first and SGPs and found that the SGP has to be introduced using the "now or never" policy. Jiang and Jain (2012b) developed a model to determine the market entry time. Two models in the marketplace were considered for this purpose: (a) a one-time sale revenue model (e.g., a TV or a car); and (b) a continuous service revenue model (e.g., a phone service subscription). Moreover, there are two types of strategies studied in Jiang and Jain (2012b): (a) phase-out, where both old and new generation products are allowed to run as long as there is a demand in the market, and; (b) substitution, where the firm discontinues the first-generation product as soon as the SGP is introduced. The Jiang and Jain (2012b) 
model includes both diffusion and substitution for one-time sales and continuous services. The model is applied to the following four scenarios: Scenario 1, phased out and one-time sale; Scenario 2, phased out and continuous service; Scenario 3, total replacement and onetime sale, and; Scenario 4, total replacement and continuous service. When Jiang and Jain (2012b) investigated the above scenarios, the main goal of the study was to maximize the total profit from all product generations by determining the optimal entry time. Jiang and Jain (2012b) found that the company introduced the second product "as early as possible" if the profit margin for the second generation was as high as that of the first generation in the continuous service model. Jiang and Jain (2012a) developed a generalized Norton-Bass model for multi-generation diffusion, which considered leapfrogging and switching. Switching defines existing adopters who adopt the new generation of the product immediately after it is introduced. Hence, an N-generation scenario was developed.

The quality of the first and SGPs make up one of the assumptions used for market entry time estimates. Kalish and Lilien (1986), and Prasad et al. (2004) focused on one generation and two generations of the product to determine the market entry time. Both studies assumed that the first-generation product was of higher quality than the SGP. However, Jiang and Jain (2012a) considered the SGP to be of improved quality compared to the firstgeneration product.

Introduction time for the new or improved product is a key element for launching a product. New product launches and their role in the success of new product development have been the most interesting field of study to determine the optimal entry time into the market. New product development comprises two stages: the actual product and the product launch. These two stages are considered categorically different, and each plays a major role in success in the market. The actual product involves components such as market demand, while the product launch deals with different stages, from concept generation to volume generation (Cooper and Kleinschmidt, 1986; Datar et al., 1997).

Lilien and Yoon (1990) and Bayus et al. (1997) studied the tradeoff between performance level and entry time. The research addressed the effects of the introduction time, the level 
of performance, and decisions of timing on the market performance of a product. For this purpose, both studies considered two groups of firms: a rushed firm and a late market. The model by Lilien and Yoon (1990) and Bayus et al. (1997) was based on the new product introduction time as well as the product performance level in a duopoly. A leader-follower structure model was used to determine the optimal entry time into the market.

\section{$2.2 \quad$ Pricing}

Introduction of a new product in the future affects the pricing policy for the new product. Different pricing policies will affect the product introduction time. Skimming and penetration are two types of pricing policies in the market. A skimming policy sets a high price initially and is used for markets which are still developing. A penetration policy sets a low price initially and is used for capturing a large market share. Robinson and Lakhani 1975, Dolan and Jeuland 1981, and Kalish 1983 assumed that the price follows the demand pattern, meaning that price increases in the introduction stage, reaches a peak, and after that, it decreases. In this policy, a low price at the introduction stage will result in early adopters having an effect on the late adopters who adopt a new product.

A market-skimming pricing policy assumes that the price of a product decreases over time (Kalish, 1983). The first reason for this is the learning curve effect (Robinson and Lakhani 1975, Dolan and Jeuland 1981, Kalish 1983). The second reason is the way consumer demand is modelled in Kalish (1983) and Horsky (1990) papers. The third reason is competitive effects (e.g., Thompson and Teng 1984, and Eliashberg and Jeuland 1986).

Based on the existing literature, optimal pricing can be obtained by the rise and fall pattern of diffusion rate, as defined by Robinson and Lakhani (1975), Kalish (1983), and Horsky (1990). In general, optimal pricing is changing based on the speed of PLC. Robinson and Lakhani (1975) studied a model to find an optimal pricing policy for a new product based on Bass (1969). Robinson and Lakhani (1975) found that the optimal pricing policy 
follows the product life cycle. However, finding such a product in the real world is very rare.

Some factors that affect the pricing of a product are learning curve, the interest rate, and word-of-mouth. Bass et al. (2000) studied different ways to capture the effect of pricing and advertising variables on the speed of a PLC. The study by Bass et al. (2005) is used in other papers with some modifications in decision variables. Krishnan et al. (1999) studied the optimal pricing policy using the generalized Bass model by incorporating the effect of price on the rate of the new product diffusion and suggested a declining pricing, or an increase-decrease pricing strategy. Furthermore, if the price sensitivity or discount rate is high, the best policy for pricing is to decrease the price. The best path for pricing is found by discounting the price coefficient. Krishnan et al. (1999) studied pricing policy when there is only one firm (monopolist). Bayus (1992) studied the effect of successive product generations on pricing policy. Monopoly markets and the constant cost for two products are assumptions by Bayus (1992). Padmanabhan and Bass (1993) investigated the maximum profit for SGPs through the planning horizon $[0, T]$ for Intel, a monopolist company. Padmanabhan and Bass (1993) assumed that the firm market has only two product generations over the planning horizon, such that the entry time for the second product generation is specified exogenously based on the experts' opinion in this field. Another assumption is that there are no competitors in the market. Kornish (2001) proposed a model for a durable-goods monopolist selling sequential products, in which the producer sells the first-generation product in the first period and SGP in the second period. Krishnamoorthy et al. (2010) provided optimal pricing and advertising policies in a durable goods category in the presence of competition. Tilson and Zheng (2014) studied effects of demand volatility on pricing decisions and proposed a model to study decisions of a durable-goods monopolist.

Some studies are focusing on the optimal pricing policy for a single product (e.g., Mahajan and Muller 1979, Norton and Bass 1987, Kalish 1988, Mahajan and Muller 1991 and Bayus 1992). Effects of price and advertising on the PLC for a single product was studied by Bass et al. (1994) and Jain and Rao (1990) and for multi-products by Jun and Park (1999) and Danaher et al. (2001). However, the Bass (1969) diffusion model did not consider the 
impact of the marketing mix variables. Mahajan and Muller (1991) addressed some issues in pricing a product such as the effect of the market expansion and technological replacement on optimal pricing policy, the effect on pricing strategy with considering integrated monopolist, and independent producers. Mahajan and Muller (1991) analyzed the pricing problem for primary and dependent products and showed that when a company produces the primary and contingent product, the price of the product is less than it is when two different companies produce those products.

Pricing and advertising are two decision variables that have to be taken into account in PIT. Bass et al. (1994) included pricing and advertising in the model and, with some modifications, this is the paper most widely used by other studies. When price and advertising remain the same from one period to another, the model reduces to the Bass (1969) model. Bass et al. (1994) studied why the Bass model fits without decision variables such as price and advertising and developed a model that could capture the effects of marketing mix variables such as pricing and advertising on demand. Jiang and Jain (2012a) introduced a closed form solution and their model reduces to the Bass model when price and advertising are changing by a constant percentage.

Finding the optimal pricing for a new generation of a product is a crucial and challenging decision because it involves dynamic demand and diffusion rate of the product. There are some studies for finding the optimal pricing that follow Bass (1969) paper such as Robinson and Lakhani (1975), and Kalish (1983). Price, advertising, promotion, personal selling, and distribution product growth are the variables studied by Robinson and Lakhani (1975) to determine how these variables would affect the Bass model. The effect of these variables on the Bass parameters, the coefficient of innovation, the coefficient of imitation, and market size have been studied by Kalish and Lilien (1986). Kalish (1983) studied market potential as a function of price and Jones and Ritz (1991) developed a model for distribution growth. Kalish (1985) concludes that price affects the market potential of a product. However, Kamakura and Balasubramanian (1988) and Jain and Rao (1990), showed that price affects the rate of diffusion rather than the market potential. 


\subsection{Advertising}

Advertising, which is a marketing mix variable, affects the demand and profit of a product. There are two advertising policies: generic advertising, static case, affects sales; brand advertising, dynamic case, affects market share. Generic advertising targets consumer beliefs about product categories, while brand advertising provides consumers with information about a brand's value. Generic advertising increases sales, and brand advertising affects the market share. According to Zhao (2000), too much advertising is not a good strategy when launching a new product. Bass et al. (2005) studied how much budget should be allocated to brand advertising and generic advertising. Some studies, such as Schmalensee (1978), and Dockner and Jorgensen (1988) have addressed optimum static advertising models.

Advertising budget is studied in papers including Fairhurst et al. (1996), and Miller and Pazgal (2007). Percentages of sales and objective sales are two common methods for determining advertising expenditures. In the percentage of sales method, a specific percentage of dollar sales is spent for advertising. In the objective method, firms spend advertising dollars to follow a specific sales level (Miller and Pazgal, 2007). Due to companies' financial limitations, percentage of sales is the method most used for advertising. The percentage of advertising budget varies for each industry. For many industries, $10 \%$ of a product's sales are used for advertising expenses.

Advertising and pricing variables, which are known as marketing mix variables, are two main variables that affect the PIT. Bass et al. (1994) model includes pricing and advertising and could capture the effects of marketing mix variables, including pricing and advertising on demand. The generalized Bass model reduces to the Bass (1969) model, when the price and advertising remain the same from one period to another period. Other authors used Bass's (1994) paper with some modification in their studies. Zhao (2000) investigated optimal time advertising and pricing strategies for introducing a new product. Zhao (2000) compared low quality and high quality products in terms of the amount of money spent on advertising.

In a competitive market, a dynamic advertising model is needed. Qi et al. (2008) proposed 
a dynamic advertising competition model in a duopoly and investigated dynamic competition in brand and generic advertising. They proposed a dynamic advertising competition model by using the optimal profit adjusting strategies. Sethi (1977) and Weng and Cheng (2008) defined four attributes for the dynamic optimum advertising model: 1) Advertising capital model, 2) Sales advertisement reaction model, 3) Individual model, and 4) The empirical study of control theory. Shadow price is an estimated price of a good or service that can be used when there is no market price. Based on the dynamic cumulative model, it can be concluded that when the shadow price of the cumulative sales volume is positive and increases with the cumulative sales volume, the effect of the price will increase, but the sales volume and advertising effect will be reduced. Furthermore, when the advertising expenses rise and can strengthen the marginal profit of the price, the optimum price and the advertising strategy of the manufacturer will increase advertising expenses and reduce the price level. When the advertising elasticity of demand increases or the monopoly power strengthens, the optimum advertising expenses will rise accordingly. However the rise of the cumulative sales volume can enhance the advertising effect, so the manufacturers should adopt a strategy to reduce the price and increase the advertising expenditure in response to price competition. Sethi et al. (2008) proposed a stochastic advertising model that incorporates the price and the advertising effects. Sethi et al. (2008) developed two models: linear demand model and isoelastic demand model and found that the value of the market and the optimal price decrease in the discount rate and increase in the advertising response remained constant. In the linear demand model, market value, the optimal price, and the optimal advertising decrease with the price sensitivity, and in isoelastic demand the same result occurs if the price of elasticity demand (i.e., a measure that shows responsiveness of the quantity demanded of a good or service to a change in its price) is low, otherwise, the opposite will occur. Weng (2009) studied non-price competitive strategies such as advertising and after-sales service and focused on the price and the advertising model. Krishnamoorthy et al. (2010) proposed a model to capture dynamic price and advertising interactions in a competitive setting. 


\subsection{Real options}

Real options is a crucial decision-making tool for an investment and has become more significant in the financial area after many years. The term "real options" was used for the first time by an MIT professor in 1977 (Myers, 1977) who then in 1984 investigated a company's strategy in the capital budgeting process. Ross (1978) used the real options valuation theory. As a project is ongoing, the risk of the project decreases; thus, using one discount rate for valuation throughout the project is not suitable (Hodder and Riggs, 1985). Traditional investment decision-making tools such as NPV may result in a faulty decision (Ross, 1995). As NPV does not consider the valuation of time flexibility in investing in a project, it undervalues the projects (Garvin and Cheah, 2004; Brandao and Dyer, 2005). Real options are applicable to many areas such as real estate, production, inventory, and interest rates (Lander and Pinches, 1998). Kellogg and Charnes (2000) used the lattice method to value the shares of high-tech companies and found that the real options valuation method is precise for this purpose. Valuing internet companies using the real option theory and capital budgeting method is described by Schwartz and Moon (2000). Gao and Jiang (2010) studied a high-risk project using the real options method. Option to defer, staged investment option, option to alter operating scale, option to abandon, option to switch, growth option, and interacting option are categories in real options, which were defined by Trigeorgis (1993).

Valuing a real asset is one of the applications of financial options in real options analysis (Trigeorgis, 2005; Rigopoulos, 2014). In the real options approach, risk-neutral valuation is used to determine the risk-adjusted discount rate that measures the risk of the project. Real options can help a manager to evaluate all the flexibilities. Expanding, contracting and switching are three different flexibilities that are used in this research.

Different papers have studied the real options approach to value an investment (Ashuri et al., 2011; Kim et al., 2012). Uncertainty is one of the challenges real options can successfully deal with compared with the traditional valuation for an investment (Bulan, 2005). 


\subsection{Gaps in the literature}

In the literature detailed in the previous sections, a number of shortcomings were found. First, the production cost is an important parameter for determining the optimum PIT for a SGP; it has not been considered in any studies focusing on sequential strategy. Second, each product has a PLC, and as the PLC of a product is changing over time, market demand will change as well. As a result, it is necessary to have a flexible production capacity for maximizing the NPV, and this has not been studied. Third, calculating how much profit a company can make in a given period when the demand is uncertain has not been considered in the existing studies.

As production cost and product demand have a close relationship, it is crucial that production cost is considered in the model to meet the market demand (Soleimani et al., 2016). Accordingly, the production cost is a major parameter for determining the optimal PIT in a manufacturing system and many of the studies did not consider the production cost in their model. Few studies have used price, demand, and cost as uncertain parameters to calculate the profit (Govindan et al., 2015). These uncertainties impose a higher degree of complexity in the model (Babazadeh et al., 2015).

The flexibility is another gap that has not been studied. To overcome market uncertainty, flexible capacity production has to be considered as a competitive strategy. The flexible capacity between two products can either be expansion, contraction, or switching. In expansion, the system is allowed to increase its capacity. In contraction, the system is allowed to decrease its capacity. In switching, the system is allowed to switch its capacity between two products. In order to have an effective production planning system, the manager must determine optimal capacity production in the early period so that decisions match output production with demand. Having a flexible system model enables manufacturing systems to produce goods at optimal capacity according to the market need (Albey et al., 2017). The ability of manufacturing systems to produce flexible output is a very complex concept, especially in machine setups and uncertain parameters (Elmaghraby, 2011). All the studies 
from a marketing point of view consider a fixed capacity system. In order to maximize the NPV, the manager must determine the optimal capacity (Bollen, 1999). A fixed capacity system can cause either excess inventory or supply shortage. Flexible capacity production can cover changes in demand over time (Ettl et al., 2012). If company resources are limited, they may share the capacity between two products. As a result, having a flexible system is needed to cover these production capacity changes over time. For each flexible system, there are some associated costs such as capacity cost and production cost. The decision to switch between production capacities needs to be made in the early period and is costly. Due to demand uncertainty, investing in a flexible system is one strategy to hedge the risk of investment (Bish and Wang, 2004). Using a flexible system, investors can reduce inventory cost and increase productivity by controlling the output (Tannous, 1996).

Cross-functional integration between marketing and manufacturing has been considered a key success factor for new PIT (Ernst et al., 2010). By integrating marketing and manufacturing aspects, the companies can enhance understanding of market information flow, which is crucial to the success of a new product introduction (Wren et al., 2000). 


\section{Chapter 3}

\section{Methodology}

This Section will provide the problem statement and describe the overall methodology used in this study. Total replacement-sequential strategy, phase out transition-sequential strategy, and phase out transition-simultaneous strategy are described. The methodology for estimating the Bass parameters is discussed.

\subsection{Problem statement}

There are two products considered: product $A$, a first-generation product (current generation product), and product $B$, a SGP (next generation product). This research deals with the first-generation product and the SGP by a monopolist who maximizes NPV over a given period of time. As this research is applicable to durable goods and the PLC of durable goods is 5 years, this research used a given period of five years (60 months). There are three significant assumptions considered in this research: (a) a monopolist market is assumed; (b) the first and second generations are from the same product family, and (c) the first and SGPs are ready to enter the market. Simultaneous and sequential are the two types of introduction time strategies considered in this research. In simultaneous strategy, both products are introduced at the same time, time zero, while in the sequential strategy, the second product will be introduced with a delay $\tau$ as shown in Figure 3.1. 


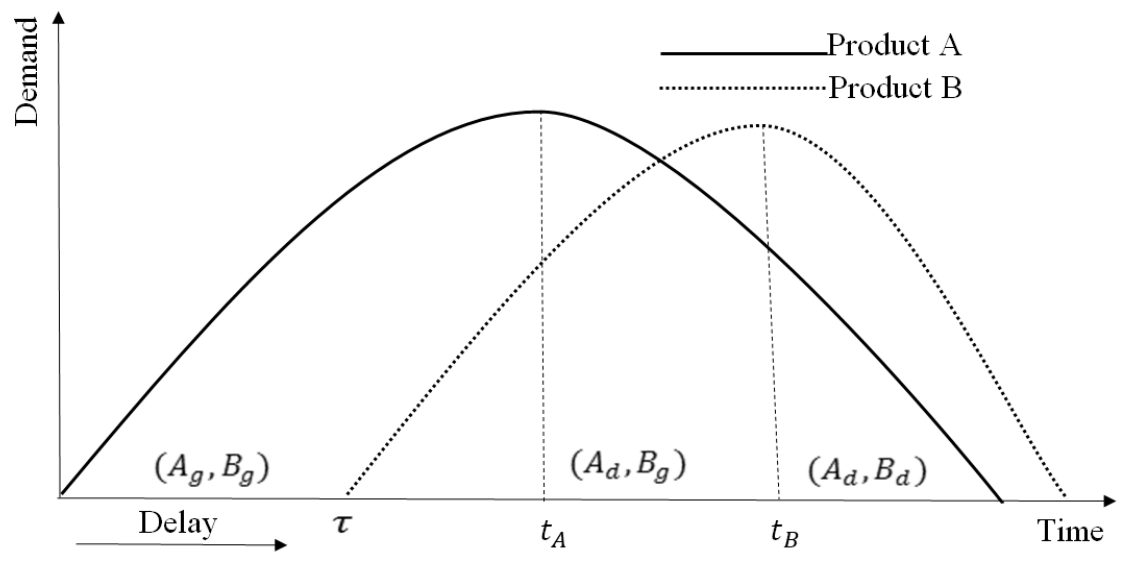

Figure 3.1: Demand profile for products $A$ and $B$

As there is uncertainty in the real world, it will affect decision making. The real options approach applies to financial options for decision-making problems. Real options are defined as a right, not an obligation, to expand, contract, or switch from one option to another. An option such as expand, contract, or switching can be used in decision-making problems when more information comes available. Having a comprehensive framework that incorporates uncertainties is vital in today's world. Using a real options approach gives flexibility to decision-makers to valuate all the possible future outcomes and make the best decision. Based on the existing literature, uncertainty is represented by a GBM. Bollen 1998 proposed a lattice approach method with regime switching probability and product life cycle is incorporated in his model with two regimes (growth and decay). In this research, a backward recursive dynamic programming method has used to valuing real options. More explanation about different value option methods with a numerical example is described in Section 3.4. This research is applicable in different decision-making levels and a manager has the flexibility to change the decisions as further information becomes available. If future situations are desired, a project may be expanded to take advantage of these situations. If further situations are undesired, a project may be contracted or abandoned. Using this research, management allows to determine if company's resources should be disbursed on a specific 
project at that future time. Figure 3.1 considers two types of products, $A$, and $B$, with stochastic two-regime PLCs. Each product contains two regimes and each regime will be defined by a geometric Brownian motion (Pindyck, 1988; Tannous, 1996). Before $t_{A}$, which is switching time for product $A$ from growth to decay regime, both products are in the growth regime, where product $A$ is introduced at time zero, and product $B$ is introduced with a delay $\tau$. As shown in Figure 3.1, product $A$ is in its decaying regime between $t_{A}$ and $t_{B}$, while product $B$ has growth regime in the same period; $t_{B}$ is the point when product $B$ switches from a growth to a decay regime. After $t_{B}$, both products will be expected to be in the decay regime. Growth and decay regimes for product $A$ will be defined as $A_{g}$ and $A_{d}$, respectively. Similarly, for product $B$, growth and decay regimes are defined as $B_{g}$ and $B_{d}$, respectively. Let $\rho_{g g}, \rho_{d g}, \rho_{g d}$ and $\rho_{d d}$ be the correlations of combined regimes $\left(A_{g}, B_{g}\right)$, $\left(A_{d}, B_{g}\right),\left(A_{g}, B_{d}\right)$, and $\left(A_{d}, B_{d}\right)$, respectively. Demand for product $A$ in growth and decay regime is denoted by $\theta_{g}^{A}$ and $\theta_{d}^{A}$, respectively. At the same time, demands for product $B$, in growth and decay regime are defined by $\theta_{g}^{B}$ and $\theta_{d}^{B}$. The drift rates for product $A$ in the growth and decay regimes are denoted by $\mu_{g}^{A}$ and $\mu_{d}^{A}$, and the drift rates for product $B$ in the growth and decay regimes are denoted by $\mu_{g}^{B}$ and $\mu_{d}^{B}$. The volatilities for product $A$ in the growth and decay regimes are denoted by $\sigma_{g}^{A}$ and $\sigma_{d}^{A}$, and the volatilities for product $B$ in the growth and decay regimes are denoted by $\sigma_{g}^{B}$ and $\sigma_{d}^{B}$. The relationship between the first and SGPs can be defined by demand parameters (e.g., drift, volatility, and correlation), which are involved in determining the best launching time for new products. As correlation is a function of drift and volatility, when the means and volatilities of demands of the first and SGPs change over time, the correlation between the demands changes accordingly.

In this research, flexible capacity between two products can either be expansion, contraction, or switching. In expansion, the system is allowed to increase its capacity. In contraction, the system is allowed to decrease its capacity. In switching, the system is allowed to switch its capacity between both products. There is a dearth of practical tools for determining the optimal introduction time (Soleimani et al., 2012). Such decision-making tools can help managers to valuate all the options to find the optimal introduction time. 
Diffusion risk (financial risk) and regime-switching risk (economic risk) are two types of risks in a regime-switching model. Diffusion risk is caused by fluctuations of market prices and regime- switching risk is due to changes in economic conditions (Siu and Yang, 2009). In order to measure the risk of a project, determining the risk-adjusted discount rate is important when used in real options valuation to calculate the NPV. For valuating a model, we need to consider two sources of risk. The first source of risk is regime-switching risk and the second source is risk from demand for the products. Investing in other projects is a way for diversifying regime-switching risk from growth to decay regime. For incorporating the risk from demand for the products if similar traded securities exist, the risk-neutral valuation can be applied. Risk-neutral valuation is a method which is used in option pricing by using the risk-adjusted growth rate (Rigopoulos, 2015). In risk-neutral valuation, the actual growth rate is replaced with the risk-adjusted rate and then cash flows are discounted at the risk-free interest rate. Estimating the risk-adjusted rate is a challenging task for a project (Garvin and Cheah, 2004). The difference between actual growth rate and risk premium is defined the risk-adjusted rate. Risk premium depends on the market price of demand risk, which measures the trade-off between risk and return (Hull, 2009). The market price of risk is related to some measure of uncertainty, such as uncertainty in demand or price volatility (Mirantes et al., 2015). The Capital Asset Pricing Model (CAPM) is a method for measuring the market price of risk. CAPM equilibrium enables us to predict the return of an asset and, by using that, managers can estimate the rate of return.

\subsection{Overall methodology}

The steps of the decision-making framework are as follows: First, a sequential timing strategy to introduce the SGP is developed; next, estimates of the stochastic demand parameters are calculated; finally, a responsive lattice model is constructed. An overall view of the research phases is shown in Figure 3.2. Input will vary depending on different considerations. When 
PLC is considered for determining the best product introduction time, the input will be demand information of PLC, which will be investigated in Chapter 4. When different pricing policies are incorporated for determining the best product introduction time, the input will be sales price and PLC data, which will be investigated in Chapter 5. When generic advertising budgeting percentage is taken into account, the input will be sales price, PLC data and advertising percentage budget, which will be investigated in Chapter 6 .

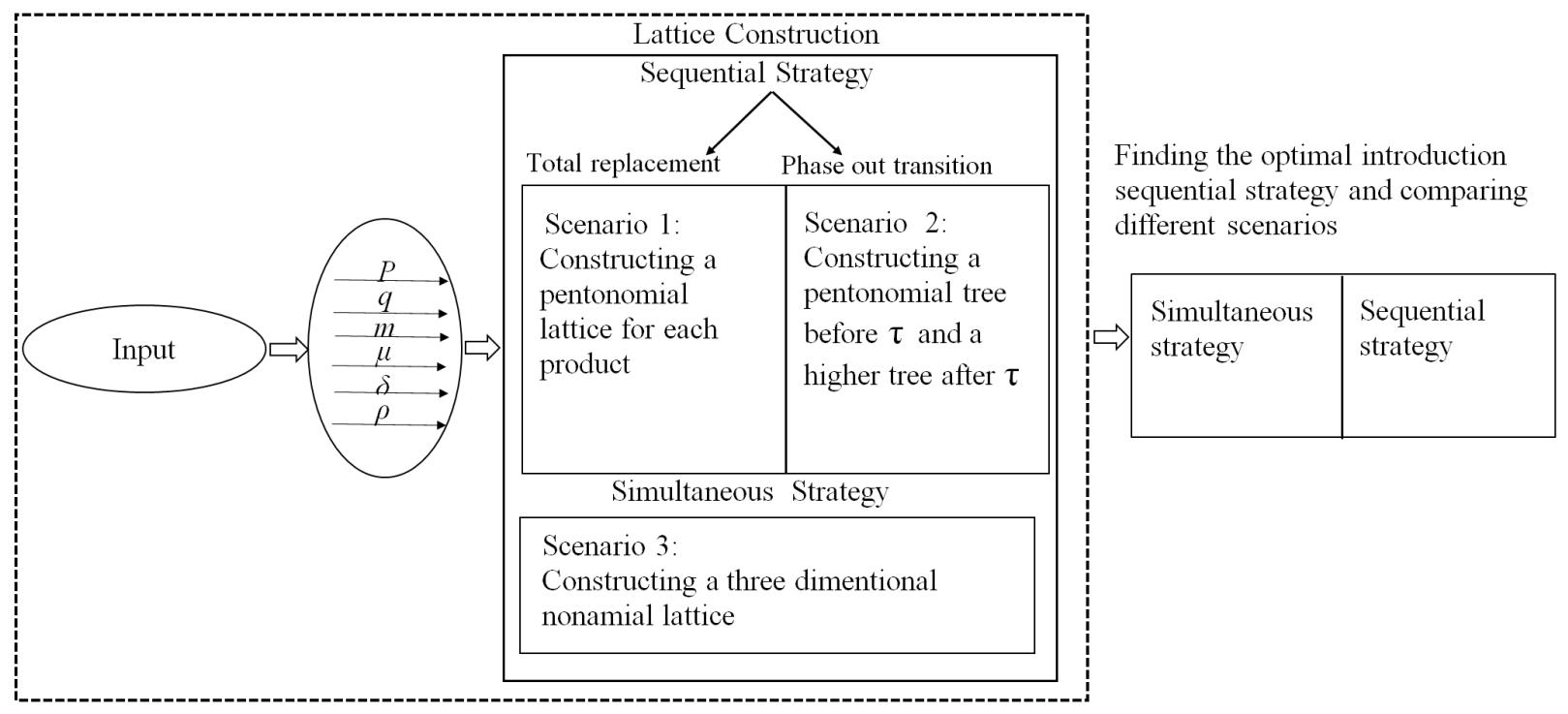

Figure 3.2: Overall framework of methodology

This research studies sequential strategy and simultaneous strategy. Sequential strategy, in which product $A$ is introduced at time zero, while product $B$ is introduced with delay $\tau$, and simultaneous strategy which products $A$, and $B$ are introduced at time zero. These two strategies are investigated in three different scenarios as follows:

Total replacement-sequential strategy (Scenario 1): In this scenario, the first-generation product will be discontinued as soon as the SGP is introduced into the market. For example, Microsoft stops selling the old version of its Windows operating system as soon as the new version is introduced (Jiang and Jain, 2012b). Product $A$ is introduced at time zero, while product $B$ is introduced with a delay $\tau$ sequentially. In this scenario, product $A$ does not exist in the market after introducing product $B$ in the market as shown in Figure 3.3. 


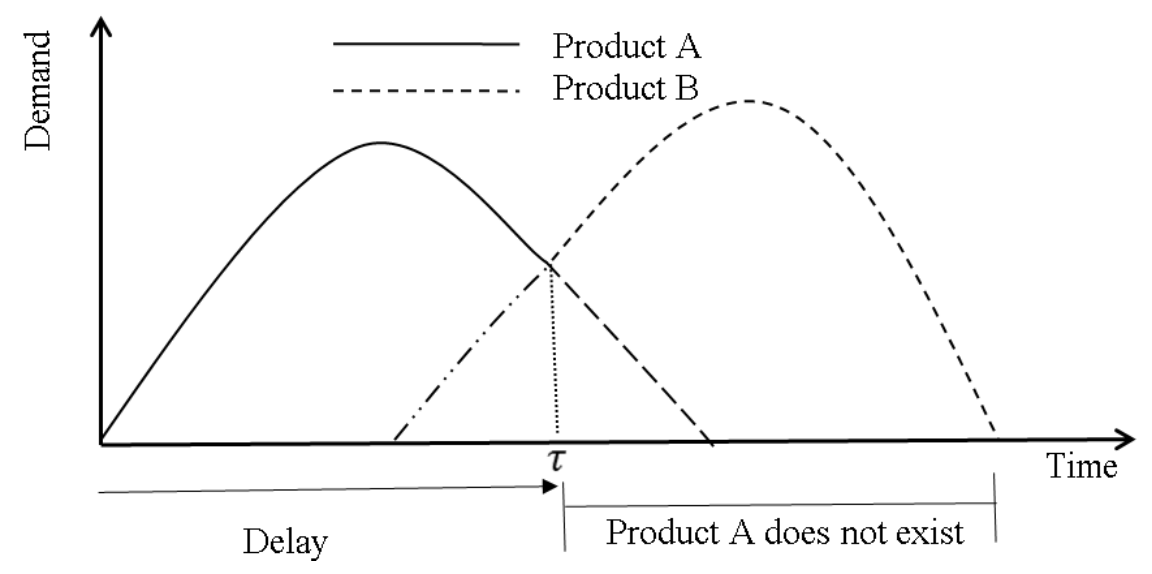

Figure 3.3: Total replacement-sequential strategy

Phase out transition-sequential strategy (Scenario 2): In this scenario, the firstgeneration product is allowed to exist in the market after the SGP is introduced; for instance, standard definition TV sets and high definition TV sets (Jiang and Jain, 2012b). Product $A$ is introduced at time zero, while product $B$ is introduced with a delay $\tau$ sequentially. In this scenario, product $A$ exists in the market after introducing product $B$ to the market as shown in Figure 3.4.

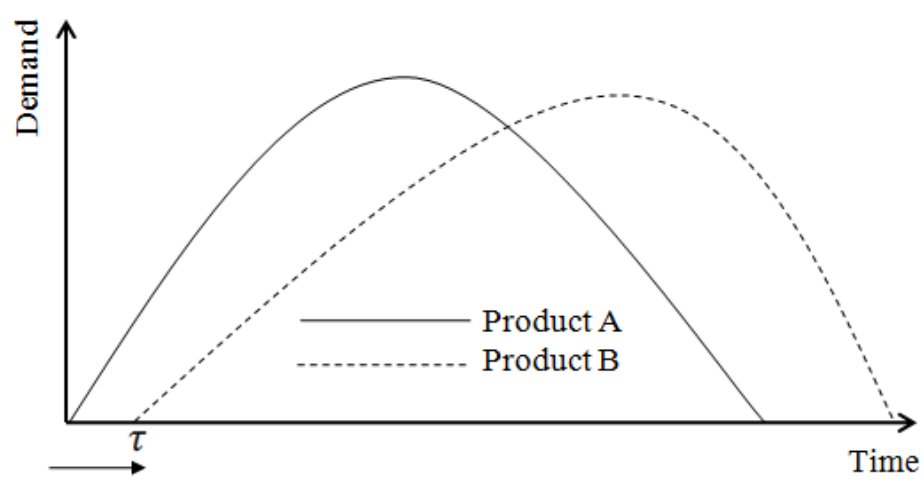

Figure 3.4: Phase out transition-sequential strategy

Phase out transition-simultaneous strategy (Scenario 3): In this scenario, the first-generation product and SGP are introduced at the same time in the market. For instance, Toyota introduced two types of SUV with different options into the market at the 
same time. In this scenario, products $A$ and $B$ are introduced simultaneously at time zero as shown in Figure 3.5. This Scenario is a special case of Scenario 2 with a delay $\tau=0$.

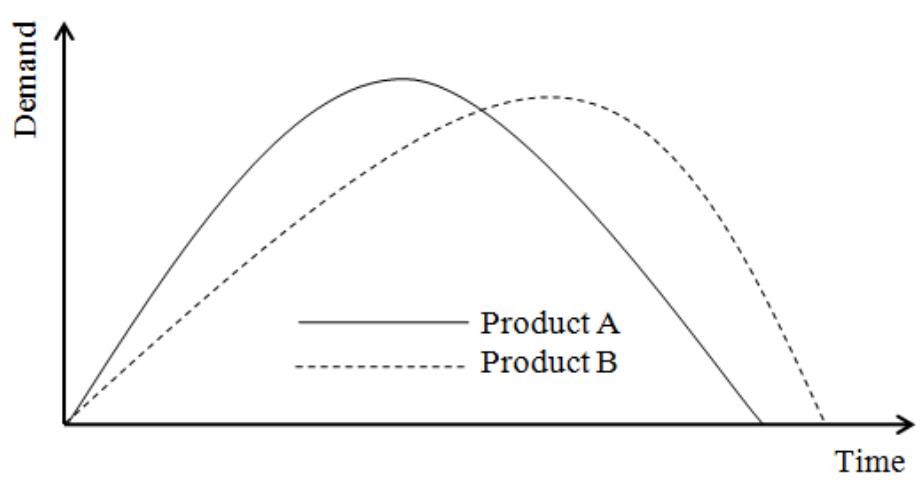

Figure 3.5: Phase out transition-simultaneous strategy

\subsubsection{Estimating $p, q$, and $m$}

Each product contains two regimes and each regime is defined by a GBM. In order to define the demand process as a GBM, we need to calculate the drift rate, volatility, and correlation of each product. For doing this the Bass model is utilized. By estimating the Bass parameters, the demand process can be defined as a GBM. The innovation coefficient or coefficient of external influence, $p$, imitation coefficient or coefficient of internal influence $q$, and; market size, $m$ are the Bass parameters. Innovators are those whose decision to adopt a product is independent of the decision of the others, and imitators are influenced by innovators over time. These Bass parameters will be estimated based on the historical data for each product by using the method explained in Bass (1969). If there is no prior data available, then we can estimate the Bass parameters by using the historical data for similar products. Mahajan and Sharma (1986) suggested a procedure for estimating the Bass parameters. Market size, the peak time of the noncumulative curve, and optimum level at peak time are the three items that need to be provided by the manager. A nonlinear least square method is used to estimate the Bass parameters.

Using the sales data from IBM during 1965-1974 period for generations 3 and 4 of their computers and nonlinear least square (NLS) method by software package R, the Bass pa- 
rameters are estimated. In the NLS method, based on the some initial values for parameters $p, q, m$, and data $\left\{x_{i}\right\}=\left\{x_{1}, x_{2}, x_{3}, \ldots x_{n}\right\}$ where $i=\{1,2,3, \ldots n\}$ and times $\left\{t_{i}\right\}$ in following equation to the data $\left\{x_{i}\right\}$ the Bass parameters $p, q$, and $m$ are estimated. For estimating the Bass parameters Equation (3.1) is used.

$$
S\left(t_{i}\right)=m f\left(t_{i}\right)=m \frac{(p+q)^{2}}{p} \frac{e^{-(p+q) t_{i}}}{\left[(q / p) e^{-(p+q) t_{i}}+1\right]^{2}} .
$$

Let

$$
S\left(t_{i}\right)=S\left(t_{i}, p, q, m\right)+e_{i}
$$

where $e_{i}$ is random error. Use initial values of $p_{j}=0.0105, q_{j}=0.0475$, and $m_{j}=440360$. The nonlinear model uses a Taylor series expansion around the parameter values i.e., $p, q$, and $m$ (Chapra and Canale, 1998).

$$
S\left(t_{i}+1\right)_{j+1}=S\left(t_{i}\right)_{j}+\frac{d S\left(t_{i}\right)_{j}}{d p} \delta p+\frac{d S\left(t_{i}\right)_{j}}{d q} \delta q+\frac{d S\left(t_{i}\right)_{j}}{d m} \delta m+e_{i}
$$

where the above given expansion has been curtailed after the first derivative. Therefore,

$$
S\left(t_{i}\right)-S\left(t_{i}, p_{j}, q_{j}, m_{j}\right)=\frac{d S\left(t_{i}\right)_{j}}{d p} \delta p+\frac{d S\left(t_{i}\right)_{j}}{d q} \delta q+\frac{d S\left(t_{i}\right)_{j}}{d m} \delta m+e_{i}
$$

Equation (3.4) can be written in the matrix form as follow:

$$
\{D\}=\left[Z_{j}\right]\{\delta A\}+\{E\}
$$


where

$$
\begin{aligned}
& \{D\}=\left\{\begin{array}{l}
S_{1}-S\left(t_{1}, p_{j}, q_{j}, m_{j}\right) \\
S_{2}-S\left(t_{2}, p_{j}, q_{j}, m_{j}\right) \\
\cdot \\
\cdot \\
\cdot \\
S_{n}-S\left(t_{n}, p_{j}, q_{j}, m_{j}\right)
\end{array}\right\} \\
& {\left[Z_{j}\right]=\left[\begin{array}{lll}
\frac{d S\left(t_{1}\right)_{j}}{d p} & \frac{d S\left(t_{1}\right)_{j}}{d q} & \frac{d S\left(t_{1}\right)_{j}}{d m} \\
\frac{d S\left(t_{2}\right)_{j}}{d p} & \frac{d S\left(t_{2}\right)_{j}}{d q} & \frac{d S\left(t_{2}\right)_{j}}{d m} \\
\cdot & \\
\cdot & \frac{d S\left(t_{n}\right)_{j}}{d q} & \frac{d S\left(t_{n}\right)_{j}}{d m}
\end{array}\right],} \\
& \{\delta A\}=\left\{\begin{array}{c}
\delta p \\
\delta q \\
\delta m
\end{array}\right\}, \\
& \{E\}=\left\{\begin{array}{c}
e_{1} \\
e_{2} \\
\cdot \\
\cdot \\
\cdot \\
e_{n}
\end{array}\right\}
\end{aligned}
$$

If we apply the least square theory to minimize the error on Equation (3.5), we will reach the following matrix equation.

$$
\left[Z_{j}\right]^{T}\left[Z_{j}\right]\{\delta A\}=\left[Z_{j}\right]^{T}\{D\}
$$


The above obtained equation is solved for $\{\delta A\}$ iteratively until the solution converges.

Table 3.1: Sales data (in thousands) from IBM for generations 3 and 4 of main frame computers

\begin{tabular}{|l||l|l|}
\hline Year & 360 family & 370 family \\
\hline 1965 & 625 & \\
1966 & 3881 & \\
1967 & 8125 & \\
1968 & 13110 & \\
1969 & 17687 & \\
1970 & 19412 & 806 \\
1971 & 17529 & 2922 \\
1972 & 14909 & 5887 \\
1973 & 10475 & 8440 \\
1974 & 8060 & \\
\hline
\end{tabular}

The Bass parameters for products $A$ and $B$ are estimated as shown in Table 3.2.

Table 3.2: Estimated Bass parameters for products $A$ and $B$

\begin{tabular}{|l||l|l|}
\hline Bass Parameters & Product $A$ & Product $B$ \\
\hline$p$ & 0.00846 & 0.0128 \\
$q$ & 0.886 & 0.9 \\
$m$ & 78561 & 40960 \\
\hline
\end{tabular}

Let $P(t)$ be the probability of purchase at time $t$ which is given by Bass (1969)

$$
P(t)=p+(q / m) Y(t),
$$

where $Y(t)$ is the total number of purchasing.

$$
[f(t)] /[1-F(t)]=P(t)=p+q F(t),
$$


where $f(\mathrm{t})$ is the likelihood of purchase at time $t$ and

$$
\begin{gathered}
F(t)=\int_{0}^{t} f(y) d y . \\
Y(t)=\int_{0}^{t} S(y) d y=m \int_{0}^{t} f(y) d y=m F(t) .
\end{gathered}
$$

$Y(t)$ is the total number of purchasing in $(0, \mathrm{t})$ period, thus sales at $t$ is

$$
S(t)=m f(t)=P(t)[m-Y(t)]=\left[p+q \int_{0}^{t} S(y) d y / m\right]\left[m-\int_{0}^{t} S(y) d y\right] .
$$

After expanding the product we have

$$
S(t)=p m+(q-p) Y(t)-q / m[Y(t)]^{2} .
$$

Also we have

$$
f(t)=[p+q F(t)][1-F(t)]=p+(q-p) F(t)-q[F(t)]^{2} .
$$

Finally, the solution is

$$
F(t)=\frac{1-e^{-(p+q) t}}{(q / p) e^{-(p+q) t}+1}
$$

then

$$
f(t)=\frac{(p+q)^{2}}{p} \frac{e^{-(p+q) t}}{\left[(q / p) e^{-(p+q) t}+1\right]^{2}},
$$

and sales at $t$

$$
S(t)=m f(t)=m \frac{(p+q)^{2}}{p} \frac{e^{-(p+q) t}}{\left[(q / p) e^{-(p+q) t}+1\right]^{2}} .
$$

The Equation (3.20) will be used in calculating the demand parameters in Chapter 4. Furthermore these equations will be modified accordingly with different considerations in Chapters 5 and 6. Details of demand probability distribution of each product for the first phase of research are described in Section 4.3; for the second phase of research details are 
described in Section 5.3, and for the third phase of research in Section 6.3.

\subsection{Value options methods}

Three main numerical methods exist in the literature. Monte Carlo simulation, finite difference methods, and lattice approach. Monte Carlo simulation is used for derivatives where the payoff depends on the history of the underlying variable, or when there are several underlying variables. Monte Carlo simulation method is not useful for decision making prior to maturity and finding the optimal policies. In Monte carlo simulation, as the number of simulation paths used to generate the stock price distribution increases, accuracy of the simulation increases.

Finite difference methods are useful when the holder has early exercise decision prior to maturity and it is useful when partial differential equation can be derived. For valuing the both American and European options, finite difference method is used. Finite difference methods are not as efficient as the Monte Carlo simulation.

Lattice is a numerical method to the valuation of derivatives when exact formula is not available, and it is used for discrete time models. Lattice is used for representing the dynamics of the underlying stochastic process. Therefore, lattice is used as a discrete representation of a continuous process. Lattice easily deals with the possibility of early exercise and it is useful for determining optimal policies and intermediate decisions. Lattice was originally introduced as binomial lattice (a.k.a two branches) developed by Cox et al. (1979). The fundamental assumption in binomial lattice is that the underlying asset price can either increase or decrease over time with certain probabilities. Branch probability, $\zeta$, and step size, $\phi$, are two parameters in each node in a binomial lattice. Branch probabilities and step sizes can be derived by matching the first and second moments (more details in Appendix C). Valuation starts at the final node and recursively back to the root node. Bollen (1998) developed pentanomial lattice (a.k.a five branches). Bollen (1998) also developed an 
approach for valuating an option when an underlying variable follows a regime-switching process with two regimes. Accordingly, one regime was defined by a binomial lattice and the other by a trinomial lattice. Since finding the optimal PIT strategies is the main goal of this research, and conducting early exercise in a given period of time becomes a key approach in finding such strategies, lattice method was used in this research. Figure 3.6 shows how a pentanomial lattice is build for three periods. Step sizes are determined using Equations (4.27-4.28). Corresponding branch probabilities are calculated using Equations (4.29-4.31). Initial demand $\theta=2.079441, \mu_{g}=0.01, \sigma_{g}=0.05, \mu_{d}=-0.01$, and $\sigma_{d}=0.015$ are given. Then for valuating, a recursive dynamic programming approach is used. Step sizes are calculated using Equations (4.27-4.28) as $\phi_{1}=0.014458$ and $\phi_{2}=0.00441$. In the next step, the exponential of initial demand is taken to retrieve the value of the demand. Figure 3.6 shows the values of initial demand at each node in a pentanomial lattice. The lattice expands with an increase in the number of nodes in accordance with $4 t-3$, where $t$ is the number of steps in the lattice. The branch probabilities are calculated using Equations (4.29-4.31) as $\zeta_{2} u=0.525216, \zeta_{2} d=0$, $\zeta_{2} m=0.4747838, \zeta_{1}, u=0.199618, \zeta_{1} m=0.483667$, and $\zeta_{1} d=0.316713$. At node 'A' the initial demand is assumed to be 2.07944 . 


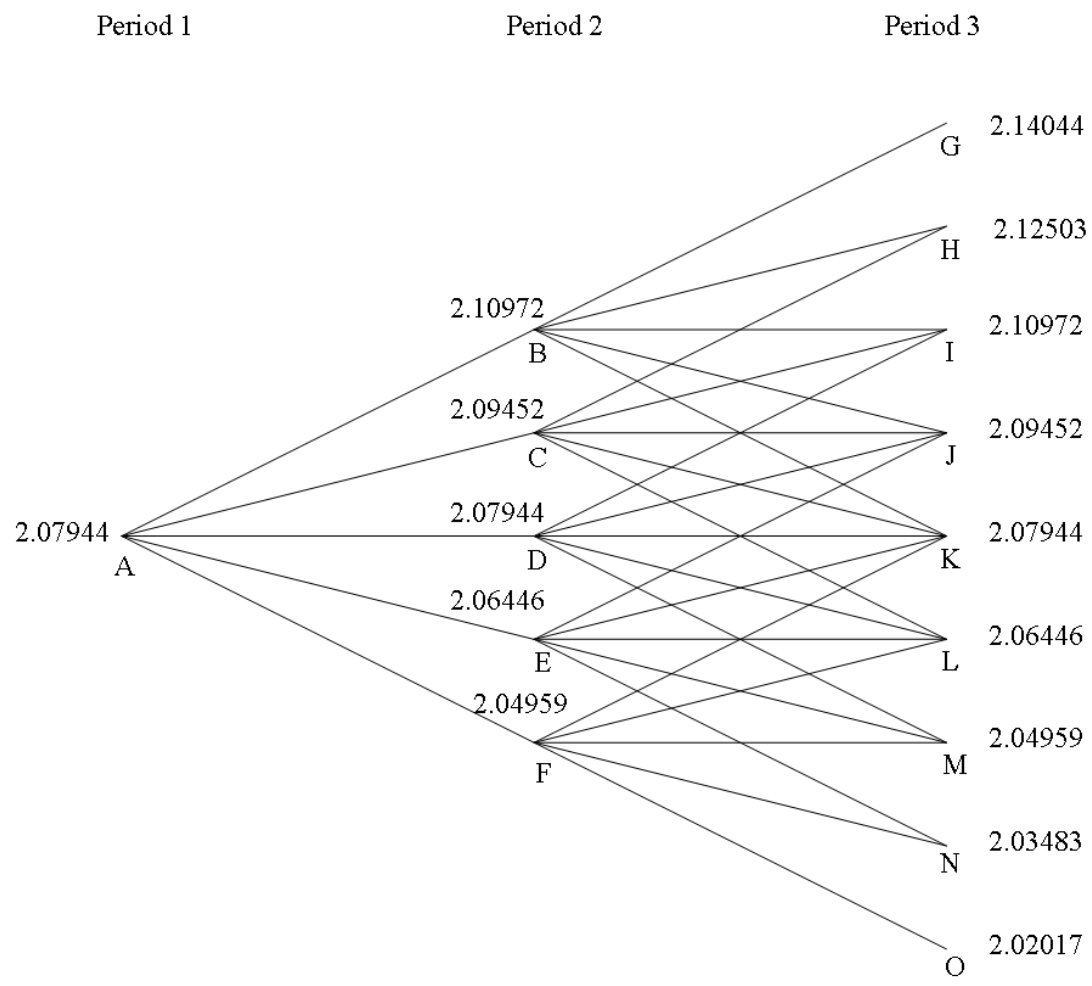

Figure 3.6: A pentanomial lattice demand value

Figure 3.7 shows the values of the profit in each node calculated by using Equations (4.38) and (4.39). 


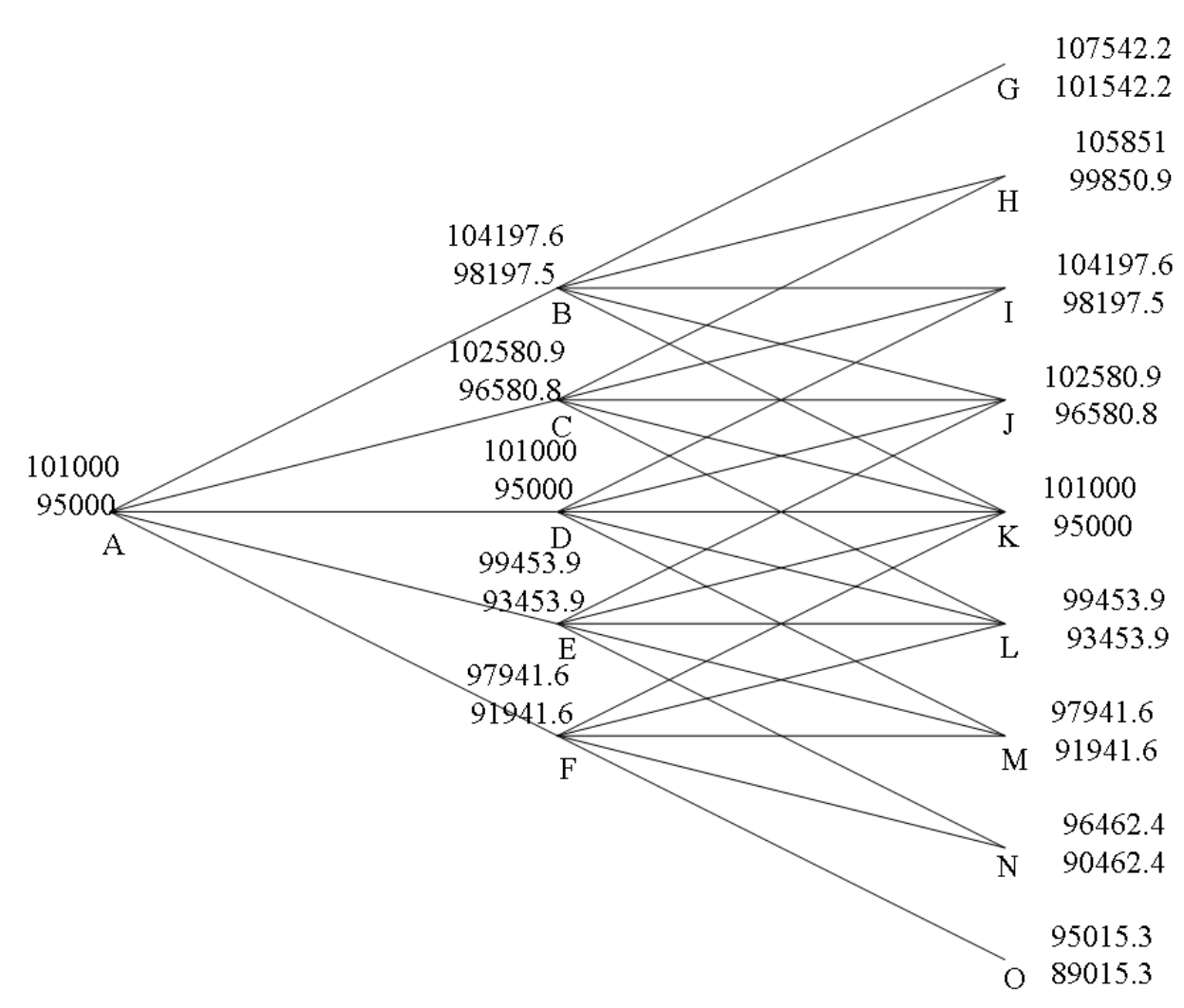

Figure 3.7: Corresponding profit in a pentanomial lattice

Dynamic programming is an optimization method used to solve complex problems by dividing them into a sequence of manageable smaller problems and it is used to value the manufacturing flexibilities. This method is recursive, where a small part is defined and then an optimum solution is determined (Bertsekas, 2015). One of the most popular techniques of dynamic programming is the use of a backward method to find the optimal path, to solve a given optimization problem. In the literature, dynamic programming is used to optimize a manufacturer's planning strategies by minimizing the cost associated with the allocation of resources and maximizing the profitability of the company.

To valuate the real options generated by the lattice, dynamic programming is used for the involved optimization purposes. The first value at each node corresponds to the profit at the capacity level of 5 , the second value refers to the profit at the capacity level of 15 . Following shows the calculating of the expected profit at each node. For example, in order to calculate 
the expected profit at node 'B' at the capacity level of 5 and without switching capacity, we get: $208126.5=104197.6+e^{(-0.05 \times 1 / 12)} \times 1 \times[0.199618 \times 105851+0.483667 \times 104197.6+$ $0.316713 \times 102580.9]+0 \times[0.525216 \times 107452.2+0 \times 104197.6+0.474783 \times 101000]$, by taking into account for the capacity level of 15 , similarly the corresponding expected value at node 'B' we get: $91305.59=104197.6+e^{(-0.05 \times 1 / 12)} \times 1 \times[0.199618 \times 99850.9+0.483667 \times 98197.5+$ $0.316713 \times 96580]+0 \times[0.525216 \times 101542.2+0 \times 98197.5+0.474783 \times 95000]+S(5,15)$. Therefore the expected profit at node 'B' for a given capacity level of 15 is $\max (208156.5$, 91305.59). Following the similar steps, we can obtain expected profit values for all the other nodes. These steps are repeated at each node and the expected profit values at each node are given in Figure 3.8.

Period 1

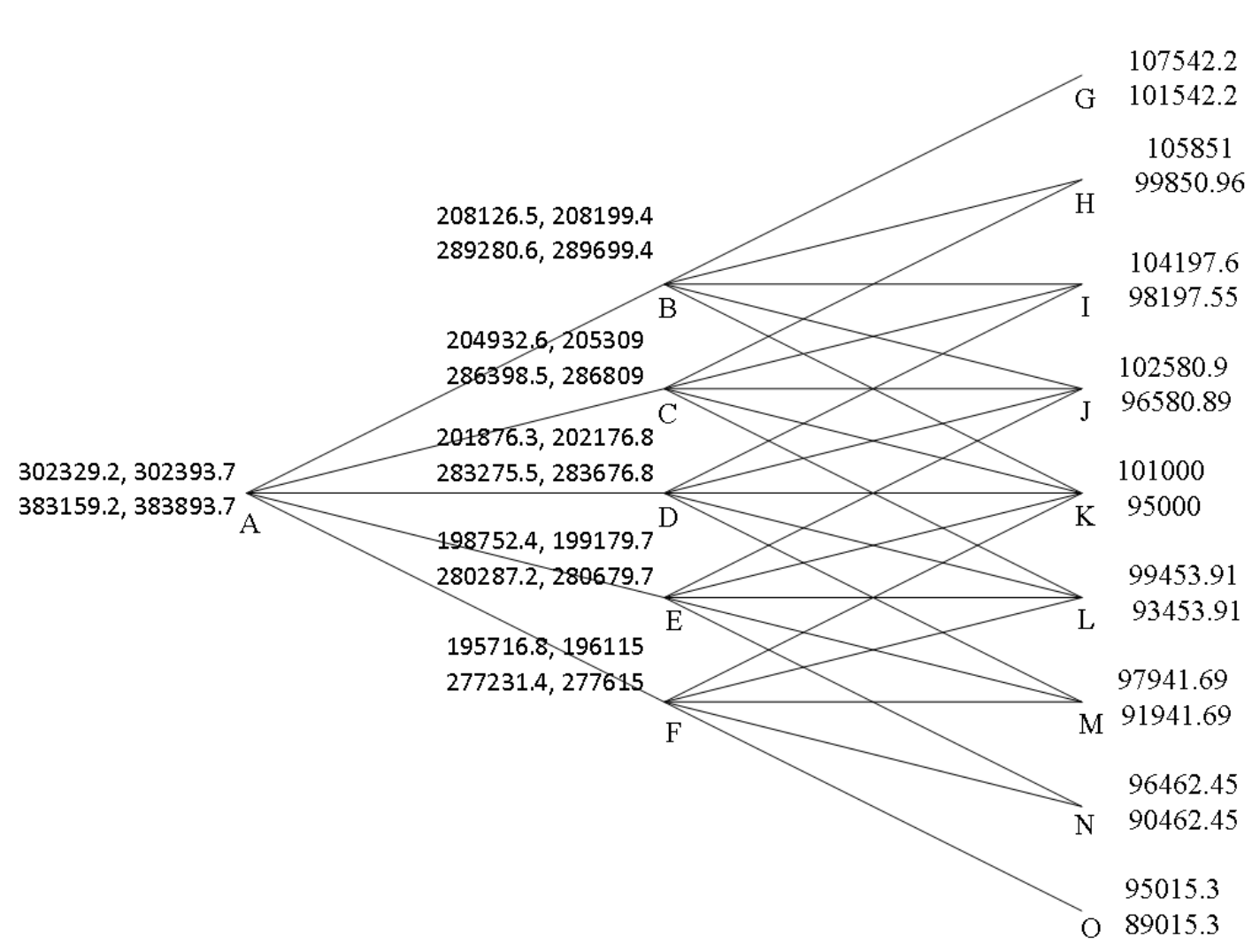

Figure 3.8: Expected profit corresponding to Figure 3.7 in a pentanomial lattice 


\section{Chapter 4}

\section{The Valuation of Product}

\section{Introduction Strategies Considering}

\section{PLC}

\subsection{Introduction}

Higher competition in the global markets, complicated customer needs, and a shorter PLC have imposed the need for continually introducing new products. Accordingly, technologies are growing quickly; new products enter the market and first-generation products are replaced by second-generation products. To stay in the market, companies are forced to improve or introduce new products. Thus, product introduction time is a big challenge for any company. For example, a six-month delay in Microsoft's Windows NT development cost them $\$ 16$ million (Zachary, 1993). Moreover, a six-month delay in shipping the HP300 series 930 computer cost Hewlett-Packard $\$ 200$ million. Even a delay in the product introduction time can decrease the market value of a firm (Hendricks and Singhal, 1997). These statistics show why product introduction time is paramount. Different product introduction time strategies may affect the company's profit. The key point in determining the best product introduction time is calculating the demand parameters. 
Product introduction time is a crucial strategic decision for many firms. This is more pronounced where the upcoming product is the development of the first-generation product, because the second-generation product introduction time strategy affects the first-generation product demand, sales, and total profit of products in a given period of time. Decision makers need to find an optimal product introduction time to maximize the profit of both products. The Norton and Bass multi-generation model is applicable in high-tech and durable products (Speece and Maclachlan, 1995). Use of a model or model results provides some information about the product such as demand, sales, and profit. An overview of the first phase of research is shown in Figure 4.1.

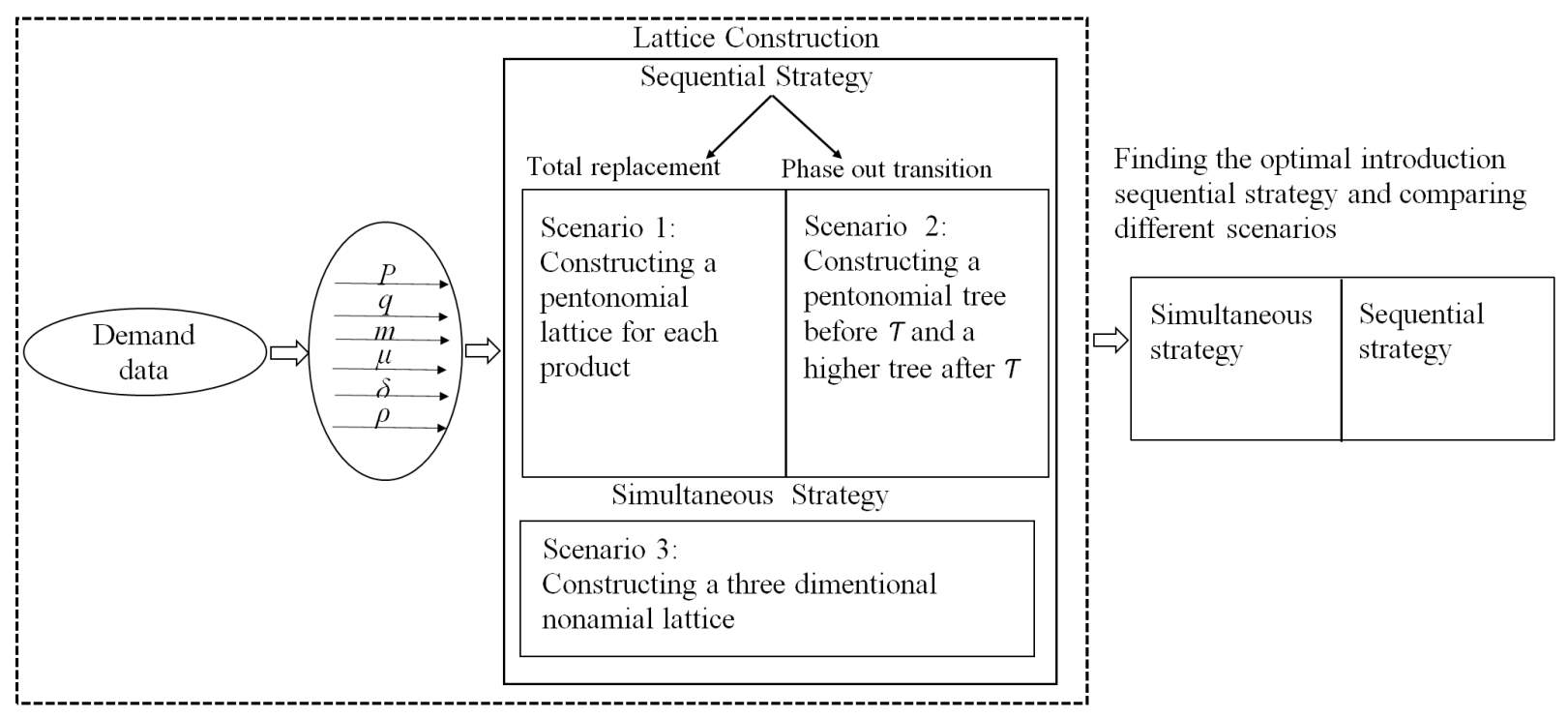

Figure 4.1: Overall framework of methodology

\subsection{Model development}

The main goal of this chapter is to find the best second-generation product introduction time which maximizes the NPV over given period in a sequential strategy. In this section, the steps to propose a first model are described. In the first step, Bass parameters innovation coefficient, $p$, imitation coefficient, $q$, and market size, $m$, are estimated using the method explained in Section 3.2.1. Then, the stochastic demand parameters (drift rate, volatility, 
and correlation) are calculated using the diffusion adoption function of each product (Jiang and Jain, 2012a). More details of estimating the stochastic demand parameters are described in Section 4.3. Finally, the lattice approach is used to model product demands; details of all the steps are described in Section 4.4. Furthermore, a dynamic programming approach is used to find the maximum NPV over a given period of time.

\subsection{Estimating the stochastic demand parameters}

Using the estimated $p, q$, and $m$ from Section 3.2.1 and following the diffusing adoption function (Jiang and Jain, 2012a), the demand probability distribution function for each product is obtained.

$$
F_{i}(t)= \begin{cases}0 & \text { if } t<0 \\ \frac{1-e^{-\left(p_{i}+q_{i}\right) t}}{\left(q_{i} / p_{i}\right) e^{-\left(p_{i}+q_{i}\right) t}+1} & \text { if } t \geq 0 .\end{cases}
$$

Where $p_{i}$ and $q_{i}$ are the coefficient of innovation and imitation, respectively for product $\mathrm{i}$ $(i \in\{A, B\})$. Using the sales data obtained from IBM from 1965 to 1974 for generation 3 and generation 4 of their computers and Equation (3.10), the Bass parameters for products $A$ and $B$ are estimated as shown in Table 4.1 .

Table 4.1: Estimated Bass parameters for products $A$ and $B$

\begin{tabular}{|l||l|l|}
\hline Bass Parameters & Product $A$ & Product $B$ \\
\hline$p$ & 0.00846 & 0.0128 \\
$q$ & 0.886 & 0.9 \\
$m$ & 78561 & 40960 \\
\hline
\end{tabular}

Calculating the stochastic demand parameters is described below. This step estimates characteristics of the PLC. Two products exist in the system, and each product could be in the growth or the decaying regime. The product demand is represented by a GBM. 
Product $A$ is modeled as follows:

$$
\theta^{A}(t)=\text { Demand of product } A= \begin{cases}\theta_{g}^{A}(t) & \text { if } t \leq t_{A} \\ \theta_{d}^{A}(t) & \text { if } t>t_{A}\end{cases}
$$

similarly, demand for product $B$

$$
\theta^{B}(t)=\text { Demand of product } B= \begin{cases}\theta_{g}^{B}(t) & \text { if } t \leq t_{B} \\ \theta_{d}^{B}(t) & \text { if } t>t_{B}\end{cases}
$$

where $t_{A}$ and $t_{B}$ are the switching time for products $A$ and $B$ from growth to decay regime, respectively.

Using historical data, we can identify characteristics of the PLC. First, the regimes are identified from historical data. Then, demand parameters can be estimated as follows. As each product is represented by a GBM, the growth regime of the product $A$ is defined as

$$
U_{\mathrm{g}}^{A}(t)=\operatorname{Ln}\left[\frac{\theta_{\mathrm{g}}^{A}(t)}{\theta_{\mathrm{g}}^{A}(t-1)}\right]
$$

and average of $U_{\mathrm{g}}^{A}(t)$ is

$$
\bar{U}_{\mathrm{g}}^{A}(t)=(1 / n) \sum_{t=1}^{n} U_{\mathrm{g}}^{A}(t)
$$

Then, drift rate, $\mu_{g}^{A}$, can be estimated as

$$
\bar{U}_{g}^{A}(t) / h .
$$

Similarly, we can define $\mu_{d}^{A}, \mu_{g}^{B}$, and $\mu_{d}^{B}$. $h$ as the time in the year between two consecutive observations. Next, $\sigma_{\mathrm{g}}^{A}$, the volatility of product A in the growth regime, can be found in Ross (1999) and Hull (2009) as

$$
\sigma_{\mathrm{g}}^{A} / \sqrt{h}
$$


where

$$
\sigma_{\mathrm{g}}^{A}=\sqrt{(1 / n-1) \sum_{\mathrm{t}=1}^{n}\left(U_{\mathrm{g}}^{A}(t)-\bar{U}_{\mathrm{g}}^{A}(t)\right)^{2}} .
$$

Estimation of other regime parameters can be calculated as above. Similarly, we can define $\sigma_{d}^{A}, \sigma_{g}^{B}$, and $\sigma_{d}^{B}$. The correlation between demands in a combined regime $\left(A_{g}, B_{g}\right)$ can be calculated as

$$
\rho_{g g}=\left[\sum_{\mathrm{t}=1}^{n}\left(U_{\mathrm{g}}^{A}(t)-\bar{U}_{\mathrm{g}}^{A}(t)\right) \cdot\left(U_{\mathrm{g}}^{B}(t)-\bar{U}_{\mathrm{g}}^{B}(t)\right)\right] /(n-1) \sigma_{\mathrm{g}}^{A} \sigma_{\mathrm{g}}^{B}
$$

similarly, for other combined regimes $\left(A_{d}, B_{g}\right),\left(A_{g}, B_{d}\right)$, and $\left(A_{d}, B_{d}\right)$ we have $\rho_{d g}, \rho_{g d}$, and $\rho_{d d}$, respectively.

$$
\begin{aligned}
& \rho_{d g}=\left[\sum_{\mathrm{t}=1}^{n}\left(U_{\mathrm{d}}^{A}(t)-\bar{U}_{\mathrm{d}}^{A}(t)\right) \cdot\left(U_{\mathrm{g}}^{B}(t)-\bar{U}_{\mathrm{g}}^{B}(t)\right)\right] /(n-1) \sigma_{\mathrm{d}}^{A} \sigma_{\mathrm{g}}^{B}, \\
& \rho_{g d}=\left[\sum_{\mathrm{t}=1}^{n}\left(U_{\mathrm{g}}^{A}(t)-\bar{U}_{\mathrm{g}}^{A}(t)\right) \cdot\left(U_{\mathrm{d}}^{B}(t)-\bar{U}_{\mathrm{d}}^{B}(t)\right)\right] /(n-1) \sigma_{\mathrm{g}}^{A} \sigma_{\mathrm{d}}^{B}, \\
& \rho_{d d}=\left[\sum_{\mathrm{t}=1}^{n}\left(U_{\mathrm{d}}^{A}(t)-\bar{U}_{\mathrm{d}}^{A}(t)\right) \cdot\left(U_{\mathrm{d}}^{B}(t)-\bar{U}_{\mathrm{d}}^{B}(t)\right)\right] /(n-1) \sigma_{\mathrm{d}}^{A} \sigma_{\mathrm{d}}^{B} .
\end{aligned}
$$

Demand parameters for products $A$ and $B$ are estimated by using estimated Bass parameters from Equation (3.10) and the method is explained above. Sales for the first and secondgeneration products are calculated by using Equations (3.18) and (3.19) and the following equations.

$$
S_{A}(t)= \begin{cases}m_{A} f_{A}(t) & \text { if } t<\tau \\ m_{A} f_{A}(t)\left[1-F_{B}(t-\tau)\right], & \text { if } t \geq \tau,\end{cases}
$$

where $\tau$ is the second-generation product introduction time. For the second-generation product we have

$$
S_{B}(t)=\left[m_{B}+m_{A} F_{A}(t)\right] f_{B}(t-\tau)+m_{A} f_{A}(t) F_{B}(t-\tau) \text {, if } t \geq \tau .
$$

Table 4.2 shows the estimated drift rate and volatility for product $A$. 
Table 4.2: Estimated demand parameters for product $A$

\begin{tabular}{|l||l|l|}
\hline Parameters & Growth regime & Decay regime \\
\hline Drift rate & 0.079705 & -0.100392 \\
Volatility & 0.036938 & 0.042652 \\
\hline
\end{tabular}

Table 4.3 shows the estimated drift rate and volatility for product $B$.

Table 4.3: Estimated demand parameters for product $B$

\begin{tabular}{|l||l|l|}
\hline Parameters & Growth regime & Decay regime \\
\hline Drift rate & 0.0526057 & -0.133522 \\
Volatility & 0.0373082 & 0.0533158 \\
\hline
\end{tabular}

Table 4.4 shows the estimated correlation of combined regimes $\left(A_{g}, B_{g}\right),\left(A_{d}, B_{g}\right),\left(A_{g}, B_{d}\right)$, and $\left(A_{d}, B_{d}\right)$.

Table 4.4: Estimated correlated demand for combined regimes

\begin{tabular}{|l|l|l|l|}
\hline$\rho_{g g}$ & $\rho_{g d}$ & $\rho_{d g}$ & $\rho_{d d}$ \\
\hline 0.44901 & -0.68195 & -0.75695 & 0.17978 \\
\hline
\end{tabular}

\subsection{Lattice approach}

Lattice is a numerical method, discrete-time model, for the valuation of derivatives when exact formulas are not available. In many studies, lattice is used for a real options approach to assess the value of a real asset (Xiong and Zhang, 2016). Lattice is basically used where exercise can take place at any time up to maturity, while a continuous time model such as Black and Scholes (1973) allows valuation of European options, where exercise might take place only on the maturity date. The basic lattice uses a construction of a binomial tree to present the different paths that the underlying asset might take over the PLC (Cox et al., 
1979). A binomial tree has two possibilities: either up, or down with probabilities for both (Ashuri et al., 2011). It is a basic lattice that reproduces uncertainty and approximates price changes as branches are increasing over a time period. By increasing the number of steps in each period, accuracy will increase (Boyle, 1988). For one product when PLC is not considered, demand of a product can be presented by using a binomial or trinomial lattice (Cox et al., 1979; Boyle, 1988) and for two products, demand can be presented by using a three-dimensional quadrinomial, pentonomial, or nonanomial lattice (Wahab, 2006). Valuing an option in a lattice starts at the end node and recurs back to the root node. In fact, the lattice is useful specifically when the holder has the option of early exercise before the maturity date. Financial options such as stocks, bonds, and exchange rates are some applications of lattice. The Wahab (2006) lattice method is used to model product demands for each product, which consists of the following steps:

Step 1: Uncorrelate the correlated GBM

The two correlated demands in a combined regime $\left(A_{g}, B_{g}\right)$ are defined as follows:

$$
\begin{aligned}
& \frac{\mathrm{d} \theta_{g}^{A}(t)}{\theta_{g}^{A}(t)}=\mu_{g}^{A} \mathrm{~d} t+\sigma_{g}^{A} \mathrm{~d} W_{t}^{A, g} \\
& \frac{\mathrm{d} \theta_{g}^{B}(t)}{\theta_{g}^{B}(t)}=\mu_{g}^{B} \mathrm{~d} t+\sigma_{g}^{B} \mathrm{~d} W_{t}^{, g}
\end{aligned}
$$

Using the Ito's Lemma, the following uncorrelated processes are based on the Hull and White (1990).

$$
\begin{aligned}
& d \ln \theta_{g}^{A}(t)=\left[\mu_{g}^{A}-\frac{\left(\sigma_{g}^{A}\right)^{2}}{2}\right] d t+\sigma_{g}^{A} d W_{t}^{A, g}, \\
& d \ln \theta_{g}^{B}(t)=\left[\mu_{g}^{B}-\frac{\left(\sigma_{g}^{B}\right)^{2}}{2}\right] d t+\sigma_{g}^{B} d W_{t}^{B, g} .
\end{aligned}
$$

Let $\chi_{1}$ and $\chi_{2}$ be such that

$$
\begin{aligned}
& \chi_{1}=\sigma_{g}^{B} \ln \theta_{g}^{A}+\sigma_{g}^{A} \ln \theta_{g}^{B}, \\
& \chi_{2}=\sigma_{g}^{B} \ln \theta_{g}^{A}-\sigma_{g}^{A} \ln \theta_{g}^{B} .
\end{aligned}
$$


According to the above two equations and using method in Appendix D

$$
\begin{aligned}
& d \Psi_{1}=\left(\left[\mu_{g}^{A}-\frac{1}{2} \sigma_{g}^{2 A}\right] \sigma_{g}^{B}+\left[\mu_{g}^{B}-\frac{1}{2} \sigma_{g}^{2^{B}}\right] \sigma_{g}^{A}\right) d t+\sigma_{g}^{A} \sigma_{g}^{B} \sqrt{2\left(1+\rho_{g g}\right)} d z_{\Psi_{1}}, \\
& d \Psi_{2}=\left(\left[\mu_{g}^{A}-\frac{1}{2} \sigma_{g}^{2 A}\right] \sigma_{g}^{B}-\left[\mu_{g}^{B}-\frac{1}{2} \sigma_{g}^{2^{B}}\right] \sigma_{g}^{A}\right) d t+\sigma_{g}^{A} \sigma_{g}^{B} \sqrt{2\left(1-\rho_{g g}\right)} d z_{\Psi_{2}} .
\end{aligned}
$$

Where $\left(\left[\mu_{g}^{A}-\frac{1}{2} \sigma_{g}^{2 A}\right] \sigma_{g}^{B}+\left[\mu_{g}^{B}-\frac{1}{2} \sigma_{g}^{2 B}\right] \sigma_{g}^{A}\right)$ and

$\left(\left[\mu_{g}^{A}-\frac{1}{2} \sigma_{g}^{2 A}\right] \sigma_{g}^{B}-\left[\mu_{g}^{B}-\frac{1}{2} \sigma_{g}^{2 B}\right] \sigma_{g}^{A}\right)$ are the instantaneous mean for combined regime $\left(A_{g}, B_{g}\right)$ in each group,

$\sigma_{g}^{A} d t+\sigma_{g}^{A} \sigma_{g}^{B} \sqrt{2\left(1+\rho_{g g}\right)}$ and $\sigma_{g}^{A} d t+\sigma_{g}^{A} \sigma_{g}^{B} \sqrt{2\left(1-\rho_{g g}\right)}$ are the instantaneous volatility for combined regime $\left(A_{g}, B_{g}\right)$ in each group, and $d z_{\Psi_{1}}$ and $d z_{\Psi_{2}}$ are the uncorrelated Wiener processes. Details of the components of the Equations (4.19-4.26) are listed in the Table 4.5. Similarly, for combined regime $\left(A_{d}, B_{g}\right)$

$$
\begin{aligned}
& d \Psi_{3}=\left(\left[\mu_{d}^{A}-\frac{1}{2}{\sigma^{2}}_{d}^{2 A}\right] \sigma_{g}^{B}+\left[\mu_{g}^{B}-\frac{1}{2} \sigma_{g}^{2 B}\right] \sigma_{d}^{A}\right) d t+\sigma_{d}^{A} \sigma_{g}^{B} \sqrt{2\left(1+\rho_{d g}\right)} d z_{\Psi_{3}}, \\
& d \Psi_{4}=\left(\left[\mu_{d}^{A}-\frac{1}{2} \sigma_{d}^{2 A}\right] \sigma_{g}^{B}-\left[\mu_{g}^{B}-\frac{1}{2} \sigma_{g}^{2 B}\right] \sigma_{d}^{A}\right) d t+\sigma_{d}^{A} \sigma_{g}^{B} \sqrt{2\left(1-\rho_{d g}\right)} d z_{\Psi_{4}} .
\end{aligned}
$$

Combined regime $\left(A_{g}, B_{d}\right)$

$$
\begin{aligned}
& d \Psi_{5}=\left(\left[\mu_{g}^{A}-\frac{1}{2} \sigma_{g}^{2 A}\right] \sigma_{d}^{B}+\left[\mu_{d}^{B}-\frac{1}{2} \sigma_{d}^{2^{B}}\right] \sigma_{g}^{A}\right) d t+\sigma_{g}^{A} \sigma_{d}^{B} \sqrt{2\left(1+\rho_{g d}\right)} d z_{\Psi_{5}}, \\
& d \Psi_{6}=\left(\left[\mu_{g}^{A}-\frac{1}{2} \sigma_{g}^{2 A}\right] \sigma_{d}^{B}-\left[\mu_{d}^{B}-\frac{1}{2} \sigma_{d}^{2 B}\right] \sigma_{g}^{A}\right) d t+\sigma_{g}^{A} \sigma_{d}^{B} \sqrt{2\left(1-\rho_{g d}\right)} d z_{\Psi_{6}} .
\end{aligned}
$$

Also for combined regime $\left(A_{g}, B_{d}\right)$ we have:

$$
\begin{aligned}
& d \Psi_{7}=\left(\left[\mu_{d}^{A}-\frac{1}{2}{\sigma^{2}}_{d}{ }_{d}\right] \sigma_{d}^{B}+\left[\mu_{d}^{B}-\frac{1}{2}{\sigma^{2}}_{d}{ }_{d}\right] \sigma_{d}^{A}\right) d t+\sigma_{d}^{A} \sigma_{d}^{B} \sqrt{2\left(1+\rho_{d d}\right)} d z_{\Psi_{7}}, \\
& d \Psi_{8}=\left(\left[\mu_{d}^{A}-\frac{1}{2} \sigma_{d}^{2 A}\right] \sigma_{d}^{B}-\left[\mu_{d}^{B}-\frac{1}{2}{\sigma^{2}}_{d}{ }_{d}\right] \sigma_{d}^{A}\right) d t+\sigma_{d}^{A} \sigma_{d}^{B} \sqrt{2\left(1-\rho_{d d}\right)} d z_{\Psi_{8} .}
\end{aligned}
$$


Table 4.5: Instantaneous mean and volatility of uncorrelated processes

\begin{tabular}{|l||l|l|}
\hline Process & Instantaneous Mean & $\begin{array}{l}\text { Instantaneous } \\
\text { Volatility }\end{array}$ \\
\hline$\left(A_{g}, B_{g}\right)_{1}$ & {$\left[\mu_{g}^{A}-\frac{1}{2} \sigma_{g}^{2 A}\right] \sigma_{g}^{B}+\left[\mu_{g}^{B}-\frac{1}{2} \sigma_{g}^{2}\right] \sigma_{g}^{A}$} & $\sigma_{g}^{A} \sigma_{g}^{B} \sqrt{2\left(1+\rho_{g g}\right)}$ \\
$\left(A_{g}, B_{g}\right)_{2}$ & {$\left[\mu_{g}^{A}-\frac{1}{2} \sigma_{g}^{2 A}\right] \sigma_{g}^{B}-\left[\mu_{g}^{B}-\frac{1}{2} \sigma_{g}^{2}\right] \sigma_{g}^{A}$} & $\sigma_{g}^{A} \sigma_{g}^{B} \sqrt{2\left(1-\rho_{g g}\right)}$ \\
\hline \hline$\left(A_{d}, B_{g}\right)_{1}$ & {$\left[\mu_{d}^{A}-\frac{1}{2} \sigma_{d}^{2 A}\right] \sigma_{g}^{B}+\left[\mu_{g}^{B}-\frac{1}{2} \sigma_{g}^{2}{ }_{g}^{B}\right] \sigma_{d}^{A}$} & $\sigma_{d}^{A} \sigma_{g}^{B} \sqrt{2\left(1+\rho_{d g}\right)}$ \\
$\left(A_{d}, B_{g}\right)_{2}$ & {$\left[\mu_{d}^{A}-\frac{1}{2} \sigma_{d}^{2}\right] \sigma_{g}^{B}-\left[\mu_{g}^{B}-\frac{1}{2} \sigma_{g}^{2}{ }_{g}^{B}\right] \sigma_{d}^{A}$} & $\sigma_{d}^{A} \sigma_{g}^{B} \sqrt{2\left(1-\rho_{d g}\right)}$ \\
\hline \hline$\left(A_{g}, B_{d}\right)_{1}$ & {$\left[\mu_{g}^{A}-\frac{1}{2} \sigma_{g}^{2}{ }_{g}^{A}\right] \sigma_{d}^{B}+\left[\mu_{d}^{B}-\frac{1}{2} \sigma_{d}^{2}{ }_{d}^{B}\right] \sigma_{g}^{A}$} & $\sigma_{g}^{A} \sigma_{d}^{B} \sqrt{2\left(1+\rho_{g d}\right)}$ \\
$\left(A_{g}, B_{d}\right)_{2}$ & {$\left[\mu_{g}^{A}-\frac{1}{2} \sigma_{g}^{2}{ }_{g}^{A}\right] \sigma_{d}^{B}-\left[\mu_{d}^{B}-\frac{1}{2} \sigma_{d}^{2}{ }_{d}^{B}\right] \sigma_{g}^{A}$} & $\sigma_{g}^{A} \sigma_{d}^{B} \sqrt{2\left(1-\rho_{g d}\right)}$ \\
\hline \hline$\left(A_{d}, B_{d}\right)_{1}$ & {$\left[\mu_{d}^{A}-\frac{1}{2} \sigma_{d}^{2}{ }_{d}^{A}\right] \sigma_{d}^{B}+\left[\mu_{d}^{B}-\frac{1}{2} \sigma_{d}^{2}{ }_{d}^{B}\right] \sigma_{d}^{A}$} & $\sigma_{d}^{A} \sigma_{d}^{B} \sqrt{2\left(1+\rho_{d d}\right)}$ \\
$\left(A_{d}, B_{d}\right)_{2}$ & {$\left[\mu_{d}^{A}-\frac{1}{2} \sigma_{d}^{2}{ }_{d}^{A}\right] \sigma_{d}^{B}-\left[\mu_{d}^{B}-\frac{1}{2} \sigma_{d}^{2}{ }_{d}^{B}\right] \sigma_{d}^{A}$} & $\sigma_{d}^{A} \sigma_{d}^{B} \sqrt{2\left(1-\rho_{d d}\right)}$ \\
\hline
\end{tabular}

Step 2: Establish two groups of uncorrelated processes and construct a two-dimensional nonanomial lattice for each group

After processing the above step, eight uncorrelated processes are obtained. Then, the eight uncorrelated processes are grouped into two by selecting one uncorrelated process from each pair. Processes $\left(A_{g}, B_{g}\right)_{1},\left(A_{d}, B_{g}\right)_{1},\left(A_{g}, B_{d}\right)_{1}$, and $\left(A_{d}, B_{d}\right)_{1}$ are considered as group 1 and $\left(A_{g}, B_{g}\right)_{2},\left(A_{d}, B_{g}\right)_{2},\left(A_{g}, B_{d}\right)_{2}$, and $\left(A_{d}, B_{d}\right)_{2}$ are considered as group 2. To determine the step size (see Appendix C for details) for each group, the following equation is used as found in Bollen (1998).

$$
\phi_{\omega j}=\sqrt{\sigma_{\omega j}^{2} h+\mu_{\omega j}^{2} h^{2}}, j=1,2,3,4
$$

In order to construct a two-dimensional nonanomial lattice, the following steps are taken: (a) Rank the step sizes in each group in ascending order; (b) Divide each step size by its 
rank, and select the maximum as $\phi_{\omega} ;(\mathrm{c})$ Adjust the step sizes as follows:

$$
\phi_{\omega_{j}}= \begin{cases}\phi_{\omega_{k}} & \text { if } j=k \\ R_{j} \phi_{\omega} & \text { if } j \neq k\end{cases}
$$

Step 3: Determine the conditional branch probabilities for each two-dimensional nonanomial lattice

Once the two-dimensional nonanomial lattice is constructed, the probability of each node is calculated by matching the first and the second moments. The branch probabilities of the regimes are calculated using Wahab (2006) thesis. The conditional branch probabilities of the trinomial lattice for process $j=1,2,3,4(j \neq k)$ are as follows:

$$
\begin{gathered}
\zeta_{\omega_{j} u}=\frac{1}{2}\left\{\frac{\zeta_{\omega j}^{2}}{\left(R_{j} \zeta_{\omega}\right)^{2}}+\frac{\mu_{\omega j} d t}{R_{j \zeta \omega}}\right\}, \\
\zeta_{\omega_{j} d}=\frac{1}{2}\left\{\frac{\zeta_{\omega j}^{2}}{\left(R_{j} \zeta_{\omega}\right)^{2}}-\frac{\mu_{\omega j} d t}{R_{j \zeta \omega}}\right\}, \\
\zeta_{\omega_{j} m}=1-\zeta_{\omega_{j} u}-\zeta_{\omega_{j} d} .
\end{gathered}
$$

$\zeta_{\omega_{j} u}, \zeta_{\omega_{j} d}$, and $\zeta_{\omega_{j} m}$ are the conditional probabilities for upward, downward, and middleward branches, respectively. If $j=k$ the branch probabilities of the binomial lattice are as follows:

$$
\begin{gathered}
\zeta_{\omega_{k} u}=\frac{1}{2}\left\{1+\frac{\mu_{\omega_{k}} d t}{\zeta_{\omega_{k}}}\right\}, \\
\zeta_{\omega_{k} d}=1-\zeta_{\omega_{k} u} .
\end{gathered}
$$

Step 4: Generate a three-dimensional lattice

Now, by using the two-dimensional nonanomial lattice developed in Step 3, a threedimensional lattice for two products, and the Cartesian products of both nonanomial lattices are generated.

Step 5: Determine the conditional branch probabilities of the three-dimensional lattice.

By multiplying the calculated probabilities of each nonanomial lattice in Step 3, the 
probabilities of the three dimensional lattice can be calculated (Hull and White, 1990) . Step 6: Restore the value of the correlated process in the three-dimensional lattice.

By using the inverse transformation of Equations (4.13) and (4.14) the value of correlated demands for combined regimes $\left(A_{g}, B_{g}\right),\left(A_{d}, B_{g}\right),\left(A_{g}, B_{d}\right)$, and $\left(A_{d}, B_{d}\right)$, respectively are obtained respectively as follows:

$$
\begin{aligned}
& \theta_{g_{t}}^{A}=\exp \left(\frac{\chi_{1}+\chi_{2}}{2 \sigma_{g}^{B}}\right), \theta_{g_{t}}^{B}=\exp \left(\frac{\chi_{1}-\chi_{2}}{2 \sigma_{g}^{A}}\right), \\
& \theta_{d_{t}}^{A}=\exp \left(\frac{\chi_{3}+\chi_{4}}{2 \sigma_{g}^{B}}\right), \theta_{g_{t}}^{B}=\exp \left(\frac{\chi_{3}-\chi_{4}}{2 \sigma_{d}^{A}}\right), \\
& \theta_{g_{t}}^{A}=\exp \left(\frac{\chi_{5}+\chi_{6}}{2 \sigma_{d}^{B}}\right), \theta_{d_{t}}^{B}=\exp \left(\frac{\chi_{5}-\chi_{6}}{2 \sigma_{g}^{A}}\right), \\
& \theta_{d_{t}}^{A}=\exp \left(\frac{\chi_{7}+\chi_{8}}{2 \sigma_{d}^{B}}\right), \theta_{d_{t}}^{B}=\exp \left(\frac{\chi_{7}-\chi_{8}}{2 \sigma_{d}^{A}}\right) .
\end{aligned}
$$

\subsection{Cost function}

For finding the best product introduction time, considering a cost function is crucial. As production cost and production demand have a close relationship, it is important to consider a cost function in the model. Price, demand, and cost are three major parameters used in some studies as uncertain parameters to calculate the profit (Govindan et al., 2015). In manufacturing systems, two types of products $A$, and $B$ were analyzed, where each product has a cost function. As shown in Figure 4.2, marginal production cost is represented by $o_{1}^{i}(i \in\{A, B\})$. This is shown by the solid line, which is constant in period $t$ before regular capacity $n_{t}^{i}$, following it increases linearly with slope $o_{2}^{i}$, up to the maximum capacity $N_{t}^{i}$. Total production cost is shown by a dotted line, which increases linearly before $n_{t}^{i}$, and after which it quadratically increases. Furthermore, $o_{3}^{i}$ shows the unit overhead cost for the 
capacity of each product $A$ and $B$. The cost function is expressed as follows (Wahab, 2006):

$$
C^{i}\left(s_{t}^{i}, N_{t}^{i}\right)=\left\{\begin{array}{lr}
o_{1}^{i} s_{t}^{i}+o_{3}^{i} N_{t}^{i} & \text { if } s_{t}^{i} \leq n_{t}^{i} \\
o_{1}^{i} s_{t}^{i}+o_{2}^{i}\left(s_{t}^{i}-n_{t}^{i}\right) s_{t}^{i}+o_{3}^{i} N_{t}^{i} & \text { if } s_{t}^{i}>n_{t}^{i} .
\end{array}\right.
$$

Where $s_{t}^{i}$ is the production quantity of product $i$ in period $t$. The profit function is explained as follows:

$$
P^{i}\left(s_{t}^{i}, N_{t}^{i}\right)=x_{t}^{i} s_{t}^{i}-C^{i}\left(s_{t}^{i}, N_{t}^{i}\right) .
$$

Where $x_{t}^{i}$ is the price of product $i$. If production quantity is within the regular capacity, the profit function will be linear, and if production quantity is more than regular capacity, the profit function will be strictly concave. This can be shown using the profit function's negative second derivative, which would have a unique maximum. Uniqueness can be shown by substituting Equation (4.38) in Equation (4.39) and taking the first and second derivatives.

$$
\hat{s}_{t}^{i}=\left(x^{i}-o_{1}^{i}+o_{2}^{i} n_{t}^{i}\right) / 2 o_{2}^{i},
$$

the optimal production quantity of product $i$ in period $t$ is:

$$
s_{t}^{i^{*}}=\min \left(\theta_{t}^{i}, \hat{s}_{t}^{i}, N_{t}^{i}\right) .
$$




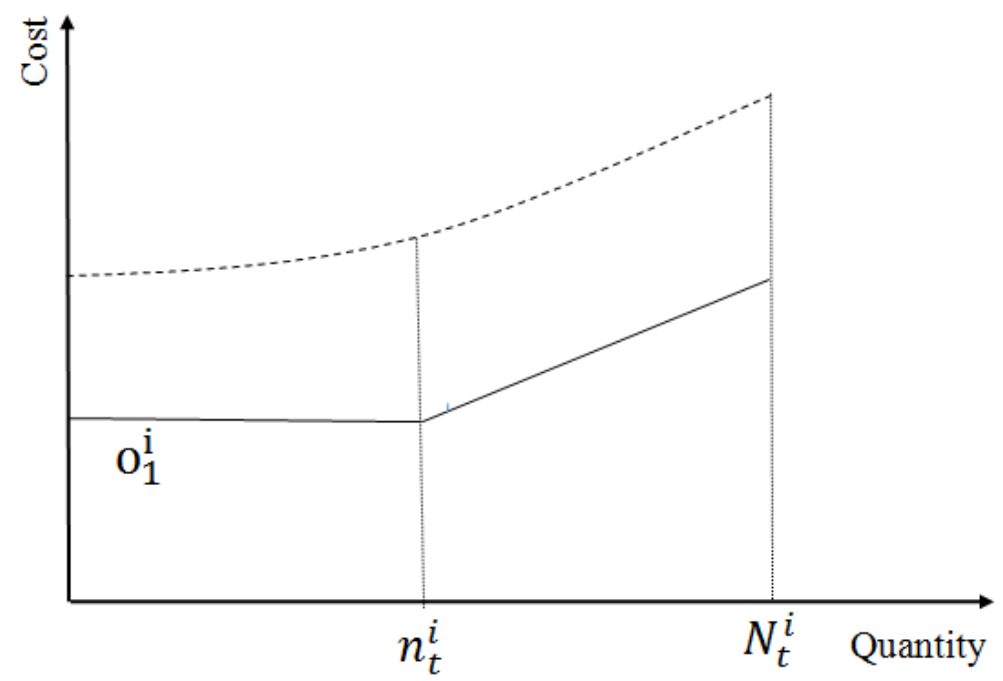

Figure 4.2: Total and marginal production cost

\subsection{Flexible systems and valuating the sequential prod- uct introduction strategy}

In the flexible capacity system, the capacity may change over the PLC to maximize total profit. Flexible capacity production is considered as a competitive strategy in uncertain markets. In each period, the capacity can expand, contract, or switch from one product to another. In expansion, the system is allowed to increase its capacity. In contraction, the system is allowed to decrease its capacity. In switching, the system is allowed to switch its capacity between two products. This flexible capacity occurs between equally spaced discrete capacity levels because the production capacity is determined by the number of operators or working machines. In order to produce optimal capacity production according to the market need, a flexible system is needed in a manufacturing system. Having a flexible manufacturing system to produce flexible output is a very complex concept, especially in machine setups and uncertain parameters (Elmaghraby, 2011). For each flexible system, there are some associated costs such as capacity cost and production cost.

By rolling back recursively from the last nodes to the root node of the lattice, valuating the 
flexible capacity system starts. Valuation for all last nodes is the final cash flow conditional on demand levels at the last nodes, and on capacity levels at prior nodes. Valuation for intermediate nodes is the sum of the current project value and the discounted expected project value in the next time period. In flexible systems, the capacity of products might change over the PLC in order to maximize the profit during the whole life cycle period (Triantis and Hodder, 1990). The NPV of the flexible systems from the period 1 to $T$ can be calculated as follows:

$N P V\left(N_{0}^{A}, N_{0}^{B}\right)=-\left(o_{4}^{A} N_{0}^{A}+o_{4}^{B} N_{0}^{B}\right)+\sum_{t=1}^{T} e^{-r t}\left\{E\left[P_{t}^{A *}\left(\theta_{t}^{A}, N_{t-1}^{A}\right)\right]+E\left[P_{t}^{B *}\left(\theta_{t}^{B}, N_{t-1}^{B}\right)\right]+Z\left(\Delta_{t}^{A}, \Delta_{t}^{B}\right)\right\}$.

Where $P_{t}^{A *}\left(\theta_{t}^{A}, N_{t-1}^{A}\right)$ and $P_{t}^{B *}\left(\theta_{t}^{B}, N_{t-1}^{B}\right)$ are the optimal profit of products $A$ and $B$, respectively. $o_{4}^{A}$ and $o_{4}^{B}$ are the cost of installing one unit of the capacity for products $A$ and $B$ at time zero, $r$ is the risk-free rate, and $T$ is the given PLC, which can also be determined based on various factors (Woodward, 1997).

In a flexible capacity system, an associated cash flow would be defined to expand, contract or switch. Let $Z\left(\Delta_{t}^{A}, \Delta_{t}^{B}\right)$ be the cash flow in the period $t$ associated with capacity adjustment decision made in the period $t-1$, where $\Delta_{t}^{A}$ and $\Delta_{t}^{B}$ are the changes in the maximum capacity level for the product $A$ and $B$, between periods $t-1$ and $t$. The fixed cost and variable costs would be associated with each capacity adjustment. The fixed costs associated with expansion and contraction for product $A$ are $f_{5}^{A}$ and $f_{6}^{A}$, respectively. The variable costs associated with expansion and contraction are $f_{1}^{i}$, which would be a fraction of the unit capacity installation cost of $o_{4}^{A}$. Similarly, product $B$ is subjected to all these costs. Expansion with one product and contraction with the other is considered capacity switching. $f_{7}^{A}$ is the fixed cost and $f_{3}^{A}$ is the variable cost of the expansion of one unit capacity expansion in capacity switching. Variable cost is a fraction of the unit capacity installation cost of $o_{4}^{A}$ for product A. Similarly, $o_{4}^{B}$ is defined for product $B$. In contrast, $f_{8}^{A}$ is the fixed cost and $f_{4}^{A}$ is the variable cost of contraction of one unit capacity contraction in capacity switching, where variable cost is a fraction of the unit capacity installation regarding product $B$, by 
$f_{8}^{B}$ and $f_{4}^{B}$. Associated cash flow in case of $\Delta_{t}^{A}, \Delta_{t}^{B} \geq 0$ pure contraction, or expansion in capacity adjustment is defined as follows:

$$
Z\left(\Delta_{t}^{A}, \Delta_{t}^{B}\right)=\sum_{i=A, B}\left\{\left(f_{5}^{i}+f_{1}^{i} o_{4}^{i} \Delta_{t}^{i}\right) I_{\Delta_{t}^{i}>0}+\left(f_{6}^{i}-f_{2}^{i} o_{4}^{i} \Delta_{t}^{i}\right) I_{\Delta_{t}^{i}<0}\right\}
$$

where the indicator function $I_{\epsilon}$, takes the value of 1 if the statement $\epsilon$ is true, else 0 . In capacity switching, $f_{7}^{A}$ and $f_{7}^{B}$ denoted fixed cost capacity and $f_{4}^{A}$ and $f_{4}^{B}$ denoted variable cost capacity for product $A$ and $B$ in expansion and contraction, respectively. $f_{8}^{A}$ and $f_{8}^{B}$

denote fixed cost capacity and $f_{3}^{A}$ and $f_{3}^{B}$ denoted variable cost capacity for product $A$ and $B$ in contraction and expansion, respectively. Associated cash flows in case of $\Delta_{t}^{A}, \Delta_{t}^{B}<0$ switching capacity adjustment are defined as follows:

$$
\begin{aligned}
Z\left(\Delta_{t}^{A}, \Delta_{t}^{B}\right) & =\left\{\left(f_{7}^{A}+f_{8}^{B}+\left(f_{3}^{A} o_{4}^{A}+f_{4}^{B} o_{4}^{B}\right)\left(-\Delta_{t}^{B}\right)+f_{1}^{A} o_{4}^{A}\left(\Delta_{t}^{A}+\Delta_{t}^{B}\right)\right\}\right. \\
& +\left\{\left(f_{7}^{A}+f_{8}^{B}+\left(f_{3}^{A} o_{4}^{A}+f_{4}^{B} o_{4}^{B}\right)\left(\Delta_{t}^{A}\right)+f_{2}^{B} o_{4}^{B}\left(-\Delta_{t}^{A}-\Delta_{t}^{B}\right)\right\}\right. \\
& +\left\{\left(f_{7}^{B}+f_{8}^{A}+\left(f_{3}^{A} o_{4}^{B}+f_{4}^{A} o_{4}^{A}\right)\left(-\Delta_{t}^{A}\right)+f_{1}^{B} o_{4}^{B}\left(\Delta_{t}^{A}+\Delta_{t}^{B}\right)\right\}\right. \\
& +\left\{\left(f_{7}^{B}+f_{8}^{A}+\left(f_{3}^{B} o_{4}^{B}+f_{4}^{A} o_{4}^{A}\right)\left(\Delta_{t}^{B}\right)+f_{2}^{A} o_{4}^{A}\left(-\Delta_{t}^{A}-\Delta_{t}^{B}\right)\right\}\right.
\end{aligned}
$$

Searching for all possible combinations of capacity levels the NPV is maximized. As the NPV of the manufacturing system is affected by regime-switching probability, Bollen's (1999) approach is used in this research to calculate the probability of switching from growth to decay regime. Accordingly, the cumulative normal distribution is used to calculate the regime-switching probability.

\subsection{Research Analysis Method}

This section provides the results of the model application into the three scenarios which were described earlier in the methodology section. In order to do this, sales data obtained from IBM from 1965 to 1974 for generation 3 and generation 4 of their computers were utilized (Mahajan and Muller, 1996). For sensitivity analysis in this research, I used a given 
period of five years (60 months or time steps), then ran the code for all time steps and next I found the maximum net present value which is the best (optimal) product introduction time. Using the IBM sales data, Bass parameters are estimated; in the next step stochastic demand parameters are calculated. Then, in a different time step, the demand parameters for each product are obtained.

\subsubsection{Initial demand and PLC}

Based on the Kalish (1985), and Ohashi (2005) research, the initial demand and drift rate of the PLC are parameters that affect the PIT. Initial demand depends on the firm's intensity of marketing activities prior to the PIT. Some activities include word-of-mouth and competition with other products in the market. Similarly, when the drift rate grows, it directly affects the demand curve by increasing it smoothly.

In Scenario 1, total replacement, Figure 4.3 shows that as the drift rate of product $A, \mu_{g}^{A}$, increases, the second-generation PIT will be delayed. As the drift rate increases, the average demand of product $A$ in growth regime increases. It is therefore expected that product $A$ lasts longer in the market, which means the second PIT will be delayed. As Figure 4.3 shows, when the second-generation PIT is 1 month, the NPV is less than $\$ 1.8$ million. When the drift rate is 0.5 , as the second PIT is delayed to 25 months, the NPV reaches a peak value of $\$ 2.4$ million. In Scenario 1, Figure 4.3 shows that when the drift rate is 0.2 , the introduction time of product $B$ is estimated at month 16. For example, the maximum NPV is obtained at month 16. Figure 4.4 shows that as the drift rate increases from 0.2 to 0.5 , product $A$ is expected to last longer in the market due to more demand for product $A$ in the market. When the drift rate is 0.5 , the introduction time of product $B$ is estimated at month 25 . This highlights that product $A$ remains in the market for 9 more months. 


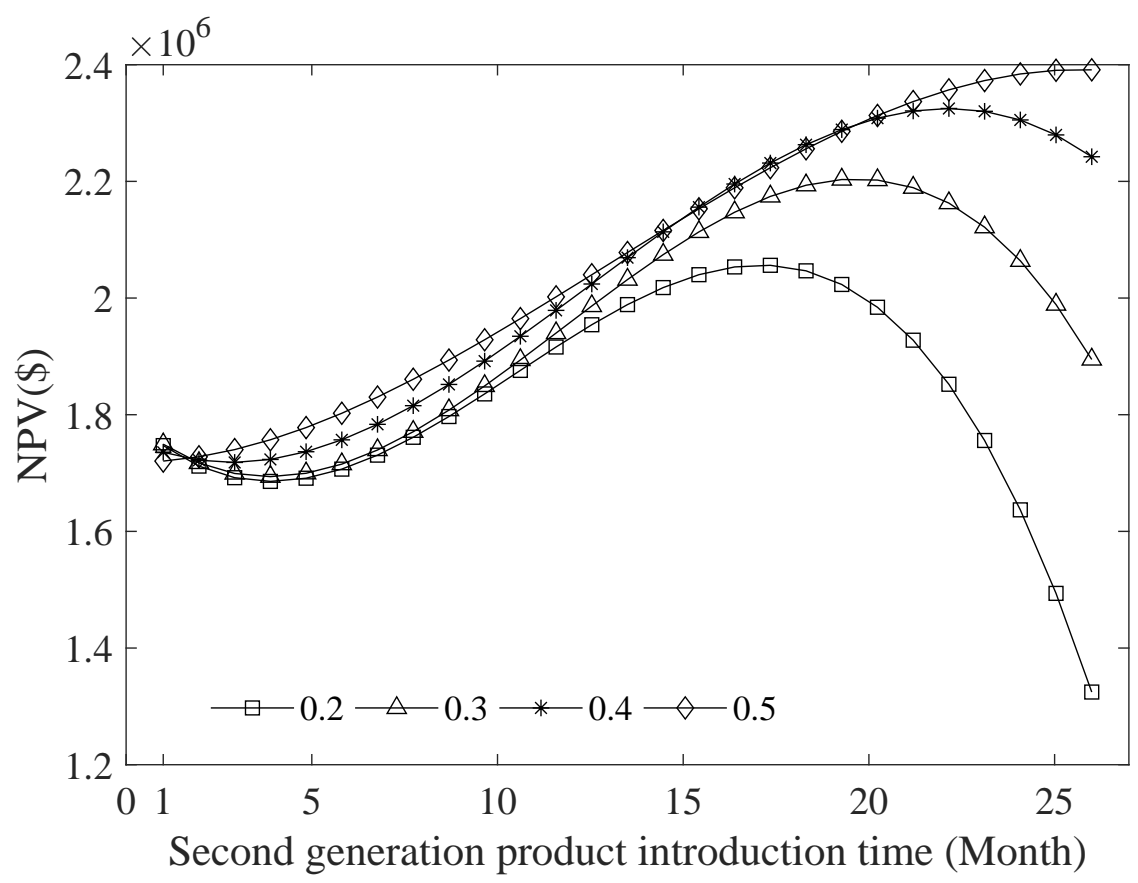

Figure 4.3: Second-generation product introduction time for different drift rates of product $A$ in growth regime-Scenario 1

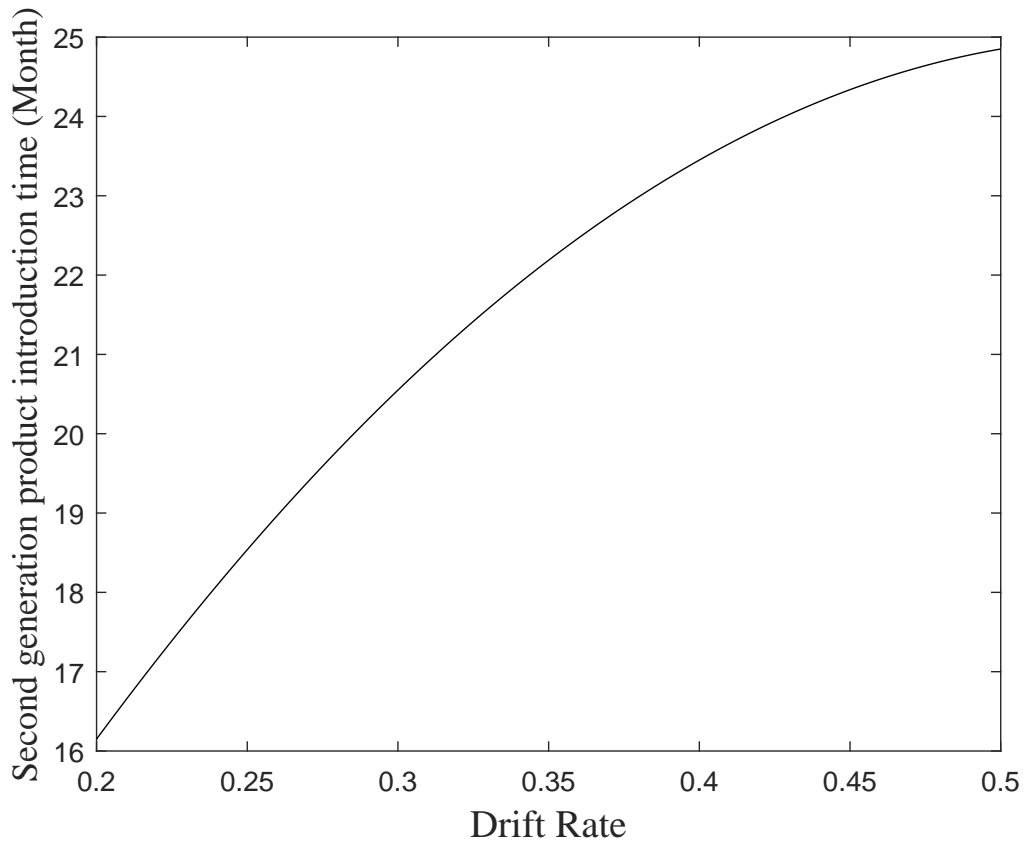

Figure 4.4: Second-generation product introduction time for different drift rates of product $A$ in growth regime-Scenario 1

It is seen from Figure 4.5 that as initial demand for product $A$ increases, the second PIT 
will be delayed. This is because of the firm's intensity of marketing activities prior to the product introduction time, which leads to increase of demand of product $A$ in the market. As a result, product $A$ lasts longer in the market, compared to when the second generation PIT is delayed. Figure 4.5 shows that when the second PIT is 1 month, the NPV is less than $\$ 1.8$ million. NPV reaches a peak value of $\$ 2.4$ million when the initial demand of product $A$ is 50 units, and the second generation PIT is delayed to 25 months. In Scenario 1, Figure 4.5 shows that when the initial demand of product $A$ is 20 units, the second PIT is estimated at month 18. For example, the maximum NPV is obtained at month 18. Figure 4.6 shows that as the initial demand is increasing from 20 to 50 units, the secondgeneration PIT increases from month 18 to month 25. Accordingly, product $A$ lasts longer in the market. This is because of the firm's intensity of marketing activities prior to the PIT.

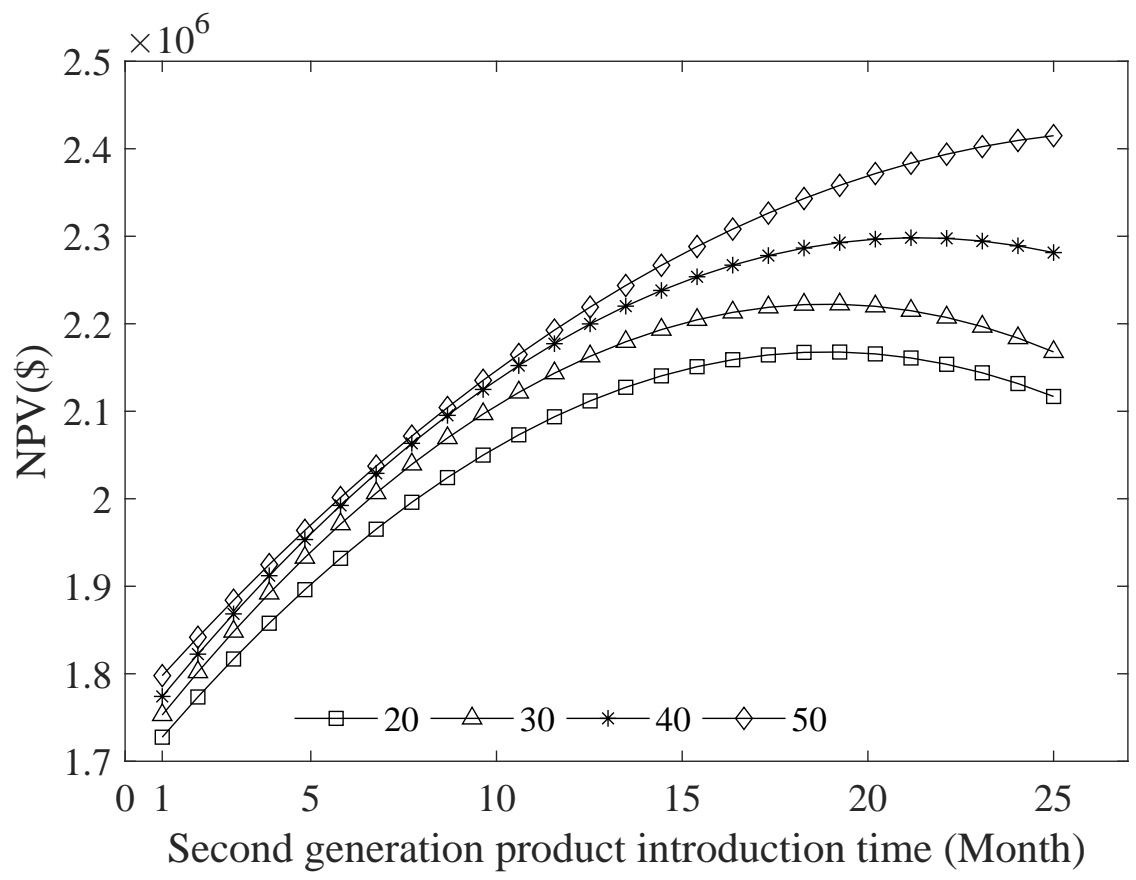

Figure 4.5: Second-generation PIT for different initial demands of product $A$-Scenario 1 


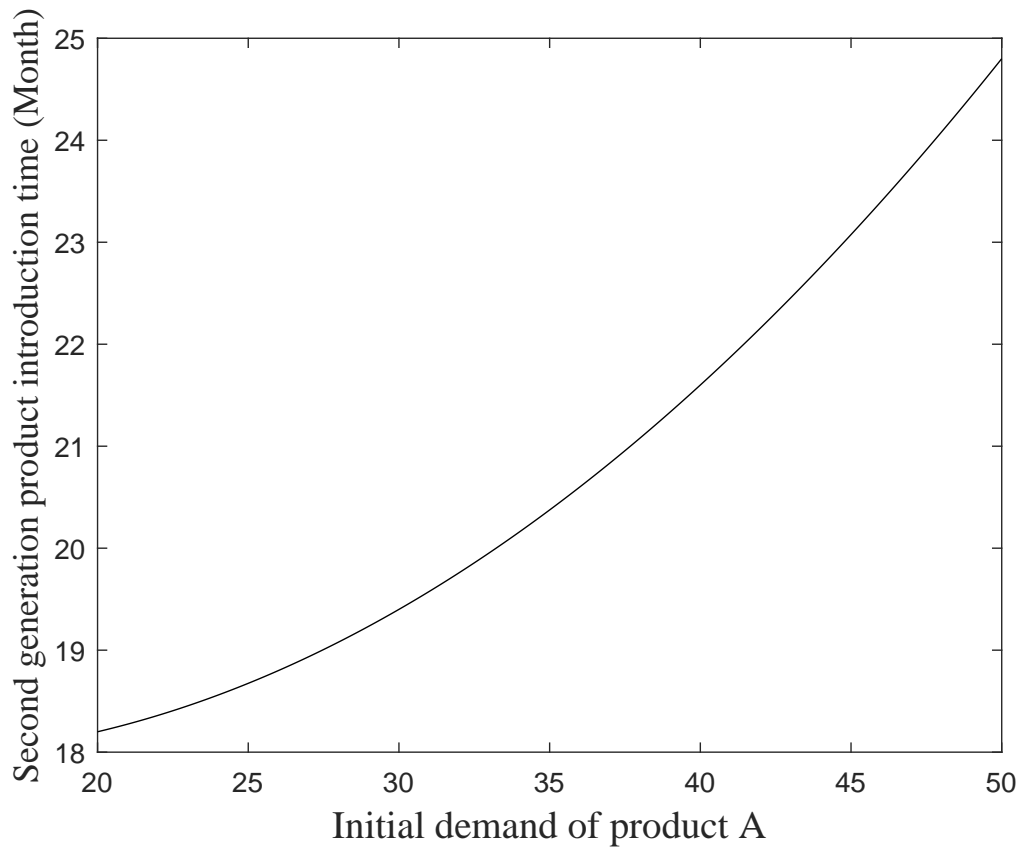

Figure 4.6: Second-generation PIT for different initial demands of product $A$-Scenario 1

In Figure 4.7, $t=0$ shows the result of Scenario 3 (Phase out transition-Simultaneous strategy), and $t=1$ to $t=17$ shows the results of Scenario 2 (Phase out transition-Sequential strategy). Figure 4.7 shows that the NPV is less than $\$ 10$ million when the second PIT is zero. NPV reaches a peak value of $\$ 62$ million when the drift rate is 0.5 , and the second generation PIT is delayed to 13 months. In Scenario 2, Figure 4.7 shows that when the drift rate is 0.1 , the second generation PIT is estimated at month 11. For example, the maximum NPV is obtained at month 11. Figure 4.8 shows that as the drift rate increases from 0.1 to 0.5 , demand for product $A$ in the market increases; therefore, second-generation PIT increases from month 11 to month 14. Accordingly, product $A$ will last longer in the market. This highlights that product $A$ remains in the market for 3 more months. 


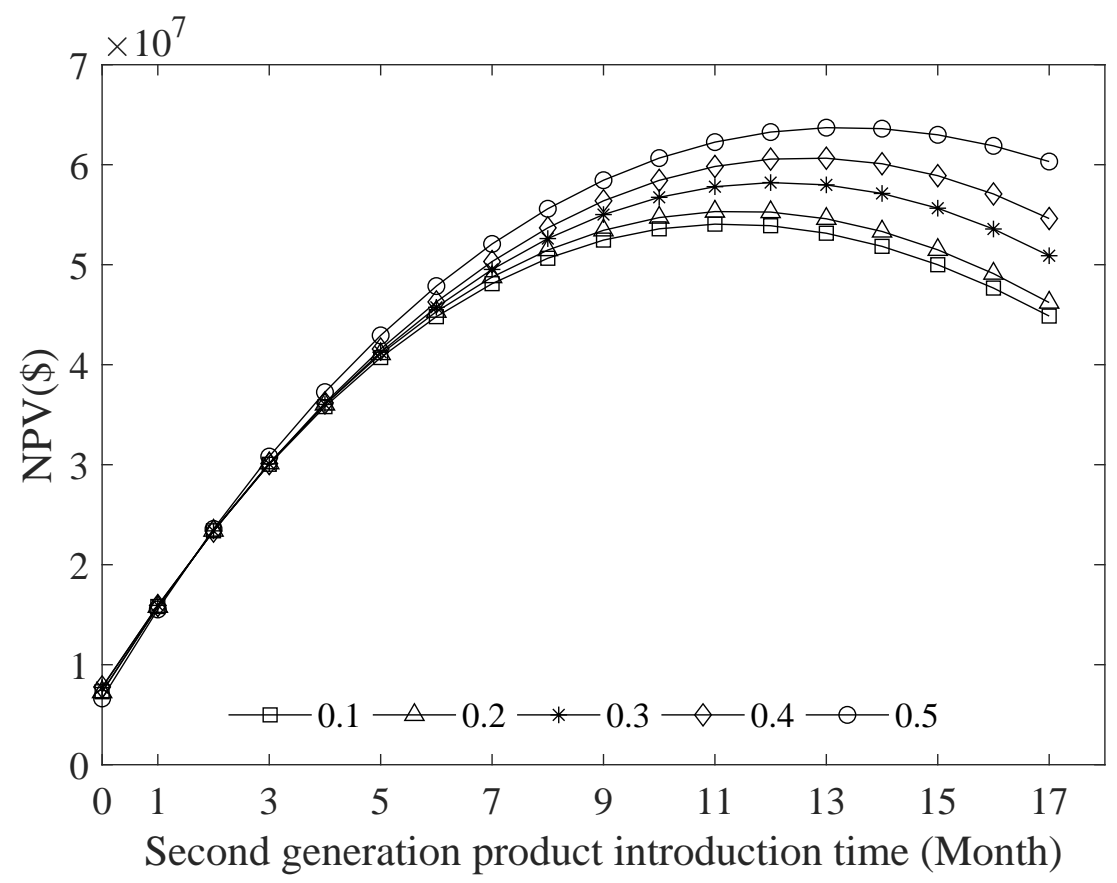

Figure 4.7: Second-generation PIT for different drift rates of product $A$ in growth regime

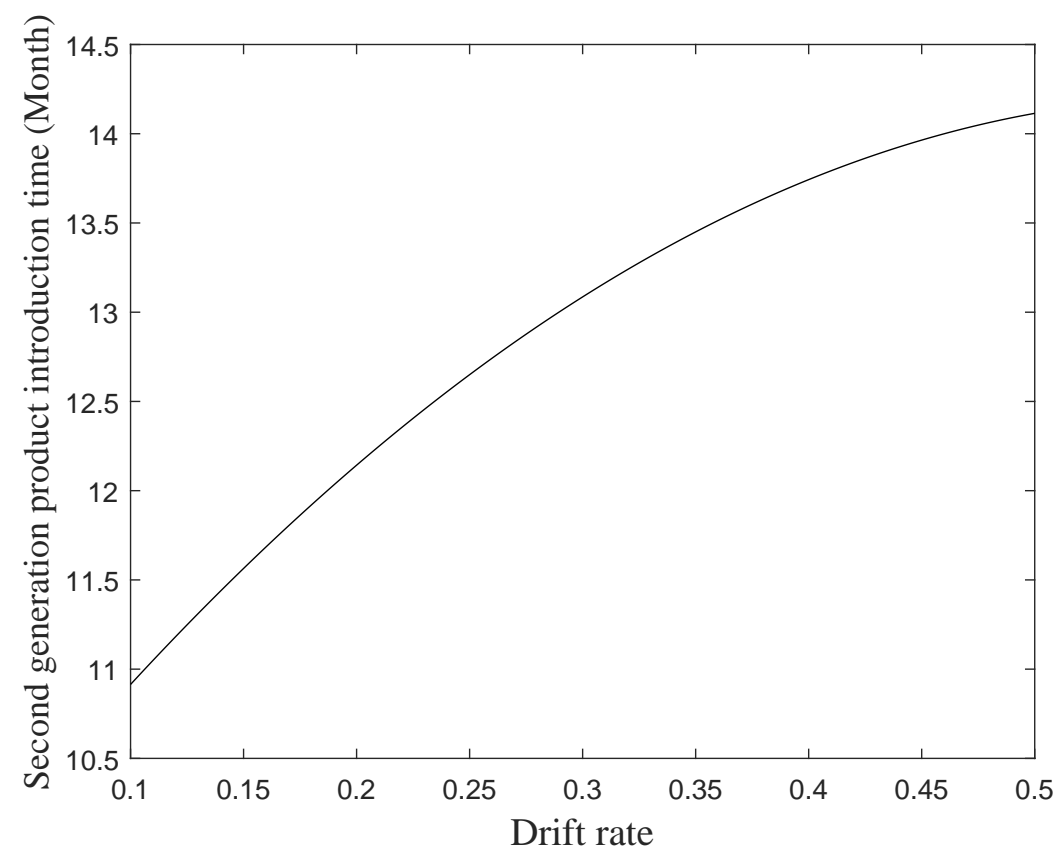

Figure 4.8: Second-generation PIT for different drift rates of product $A$ in growth regime

In Figure 4.9, $t=0$ shows the result of Scenario 3, and $t=1$ to $t=17$ shows the results of Scenario 2. NPV is less than $\$ 100$ millions when the second generation PIT is zero. NPV 
reaches a peak value of $\$ 240$ million when the initial demand of product $A$ is 50 units, and the second generation PIT is delayed to 9 months. In Scenario 2, Figure 4.9 shows that when the initial demand of product $A$ is 10 units, the second generation PIT is estimated at month 8. For example, maximum NPV is obtained at month 8. Figure 4.10 shows as the initial demand increases from 10 to 50 units, second-generation PIT increases from month 8 to month 9. Accordingly, product $A$ is expected to last longer in the market. This highlights that product $A$ remains in the market for one more month.

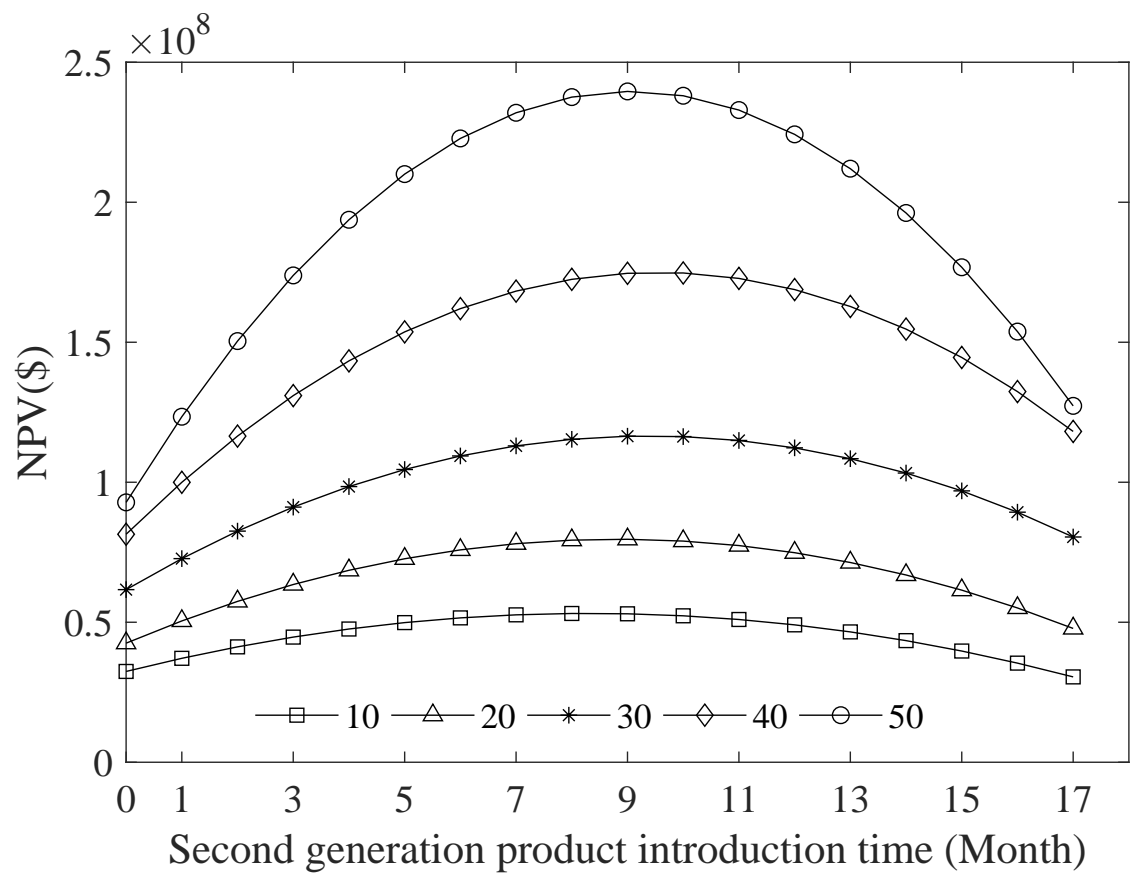

Figure 4.9: Second-generation PIT for different initial demand of product $A$ 


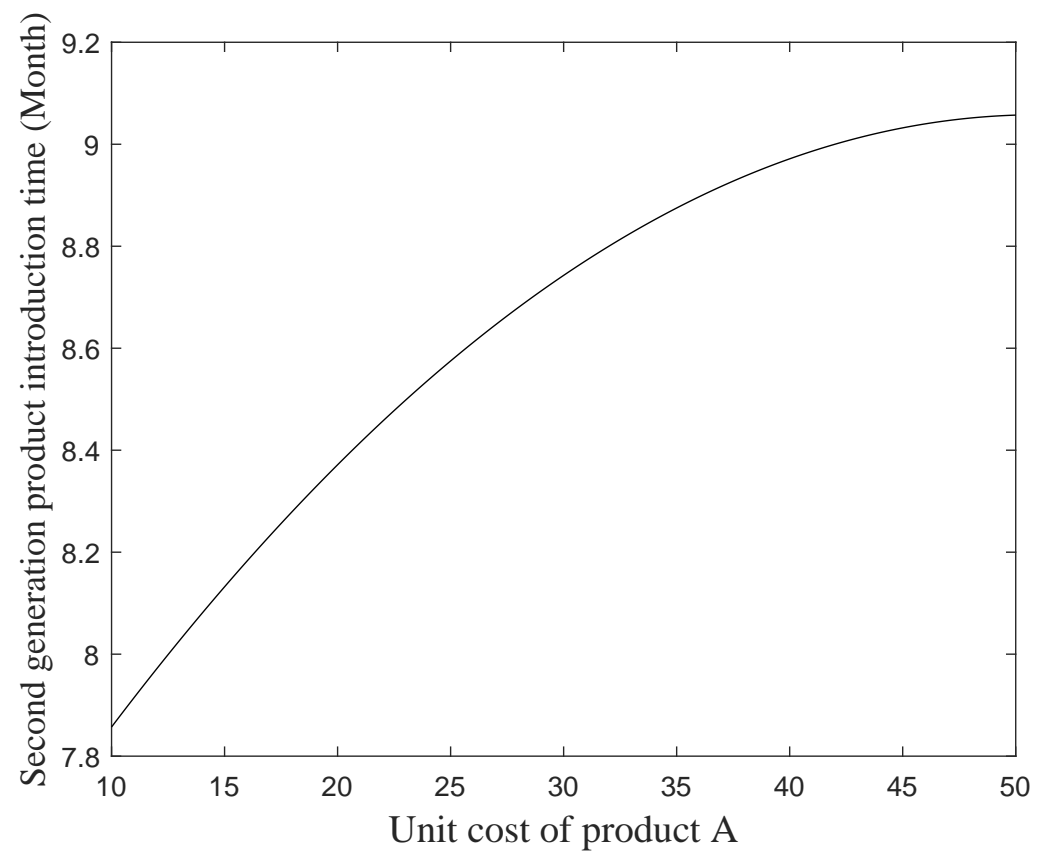

Figure 4.10: Second-generation PIT for different initial demand of product $A$

\subsubsection{Effect of the profit margin on the second-generation PIT}

Based on the research by Prasad et al. (2004), firms that introduce different generations of the same products into the market should consider the cannibalization effect as well as the tradeoff between the revenue generated from the first-generation product versus the projected revenue from the second-generation product (Hendricks and Singhal, 1997). Hendricks and Singhal (1997) assumed that both products were ready to be introduced into the market. Due to the small cannibalization effect of the high-margin product generation, the firm chooses to first launch the product with the higher profit margin. As a result, decreasing the first generation product cost produced a higher profit.

In Scenario 1, Figure 4.11 shows that the second-generation PIT will be delayed as the unit cost of product $A$ decreases. This is because as the cost decreases, the NPV increases. As a result, the second-generation PIT is delayed. Figure 4.12 shows that as the unit cost of product $A$ increases from 30000 to 60000 , demand for product $A$ decreases, then secondgeneration PIT decreases from month 18 to month 15. 


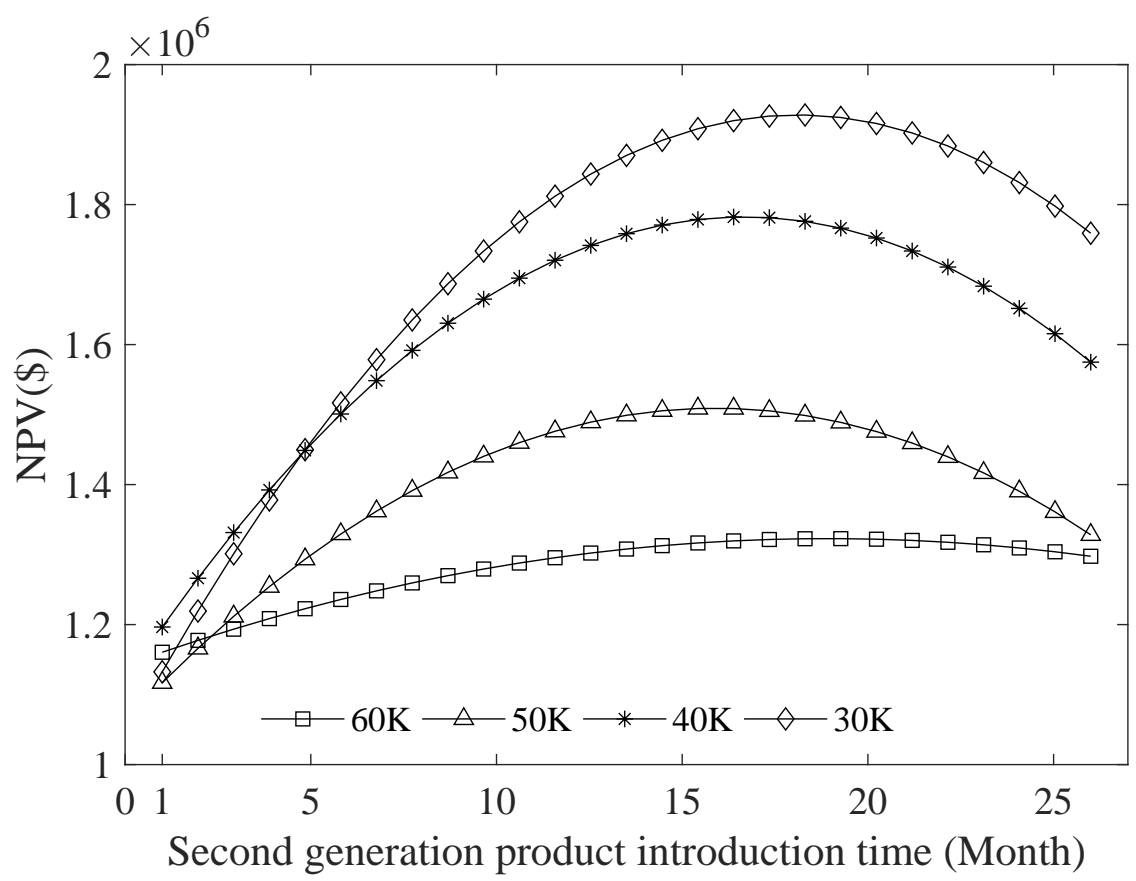

Figure 4.11: Second-generation PIT for different unit costs of product $A$

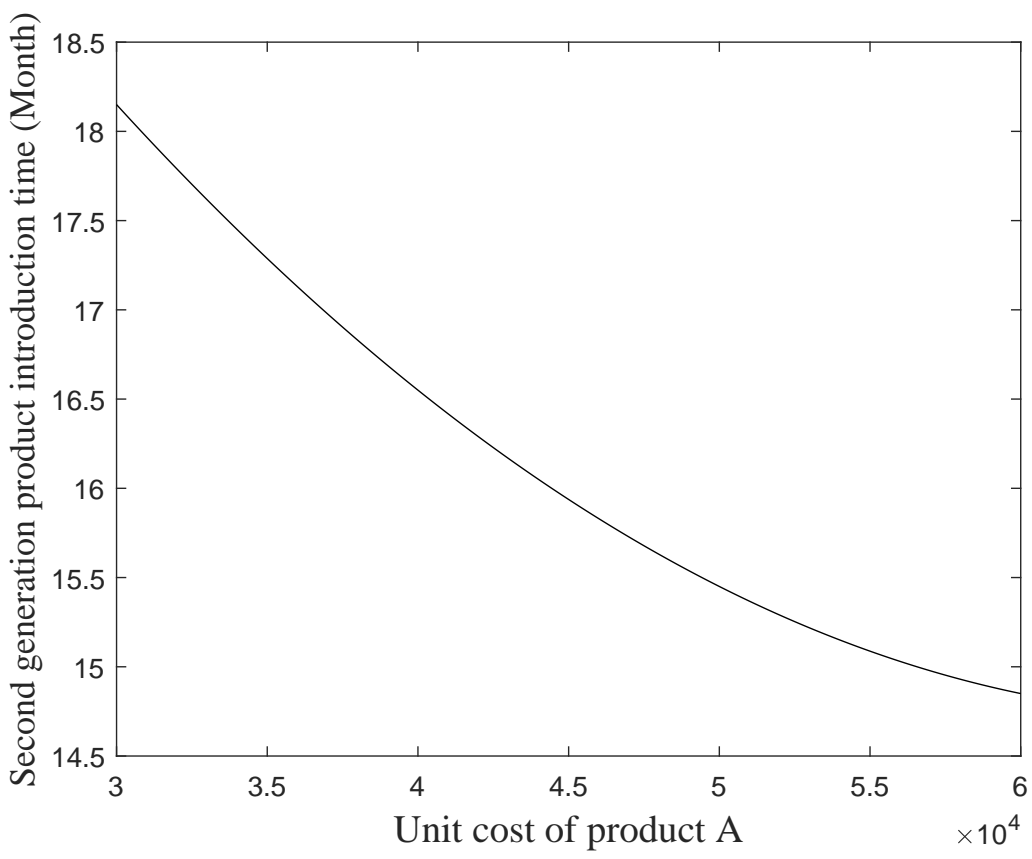

Figure 4.12: Second-generation PIT for different unit costs of product $A$

In Figure $4.13, t=0$ shows the result of Scenario 3 , and $t=1$ to $t=17$ shows the results 
of Scenario 2. Figure 4.13 shows that the NPV is less than $\$ 10$ million when the second PIT is zero. NPV reaches a peak value of $\$ 48$ million when the unit cost of product $A$ is $\$ 20000$ and the second PIT is delayed to 12 months. In Scenario 2, Figure 4.13 shows that when the unit cost of product $A$ is $\$ 60000$, the second PIT is estimated at month 10. For example, the maximum NPV is obtained at month 10. Figure 4.14 shows as the unit cost of product $A$ decreases from $\$ 60000$ to $\$ 20000$, demand for product $A$ increases. Therefore, second-generation product introduction increases from month 9 to month 11. Accordingly, product $A$ is expected to last longer in the market. This highlights that product $A$ remains in the market for 2 more months.

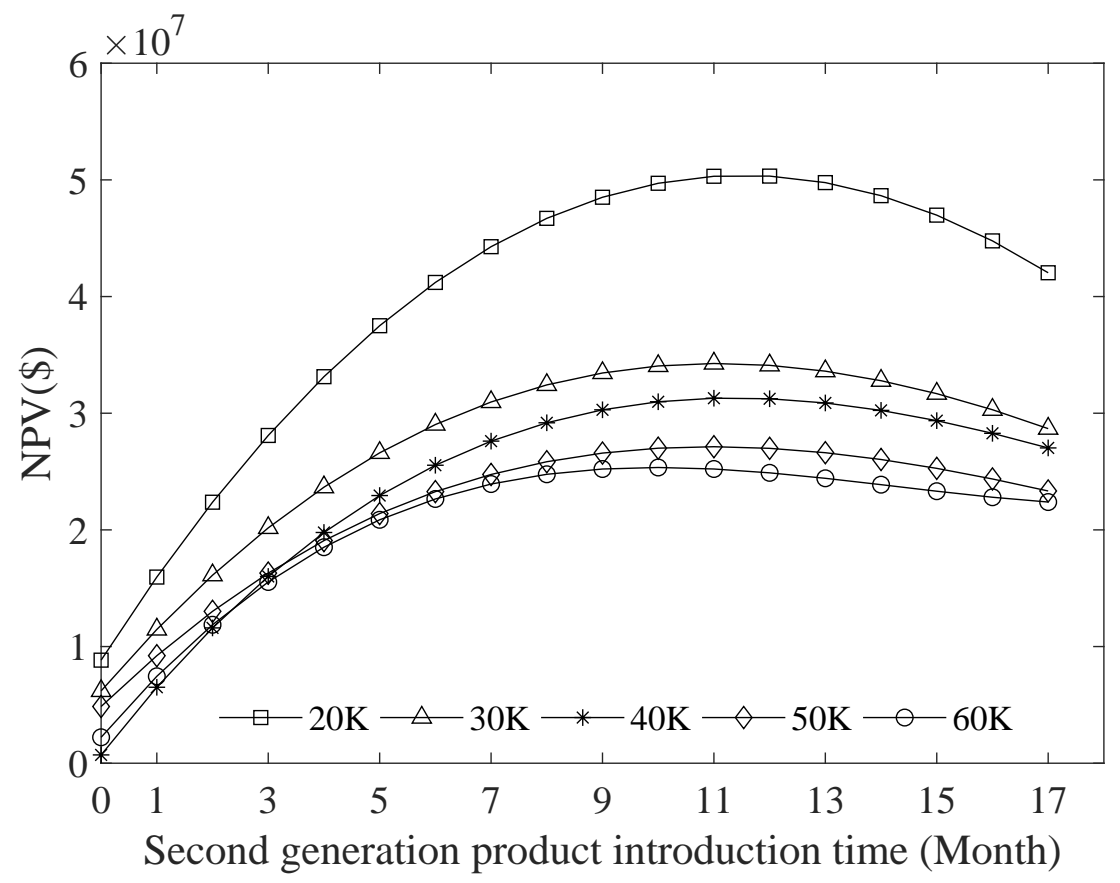

Figure 4.13: Second-generation PIT for different unit costs of product $A$ 


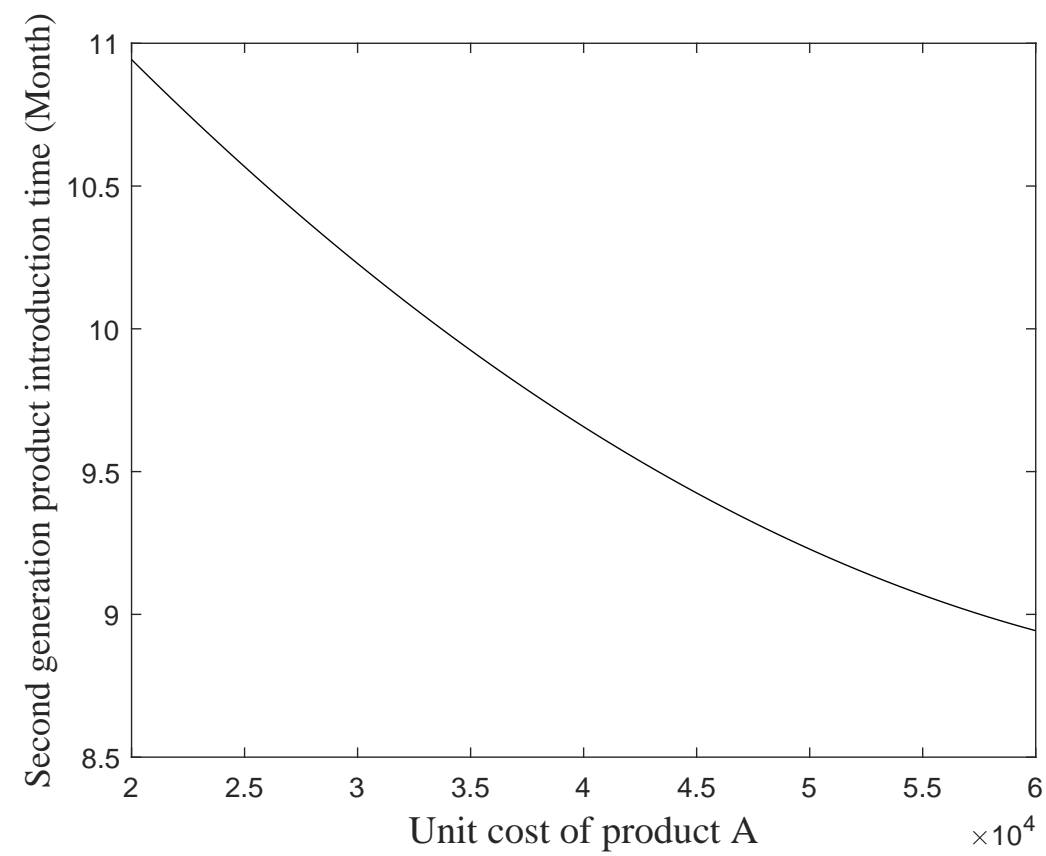

Figure 4.14: Second-generation PIT for different unit costs of product $A$

\subsubsection{Effect of the demand uncertainty on the second-generation PIT}

Because of diffusion and substitution, the interrelationship of sales of two products will affect optimal market entry timing for first- and second-generation products (Norton, 1989). Based on the discussions provided in the methodology section, the effects of diffusion (Jiang and Jain, 2012a) and substitution on the PIT were thoroughly analyzed in the three described scenarios.

In Scenario 1, Figure 4.15 shows that the second PIT will be delayed as the volatility of product $A, \sigma_{g}^{A}$, increases. This is because as the volatility of product $A$ increases, it is expected that the demand variation of product $A$ increases. Therefore, product $A$ lasts longer in the market and the second PIT is delayed. Figure 4.16 shows that as the volatility of product $A$ increases from 0.2 to 0.5 , second-generation PIT increases from month 17 to month 19. This highlights that product $A$ remains in the market for two more months. 


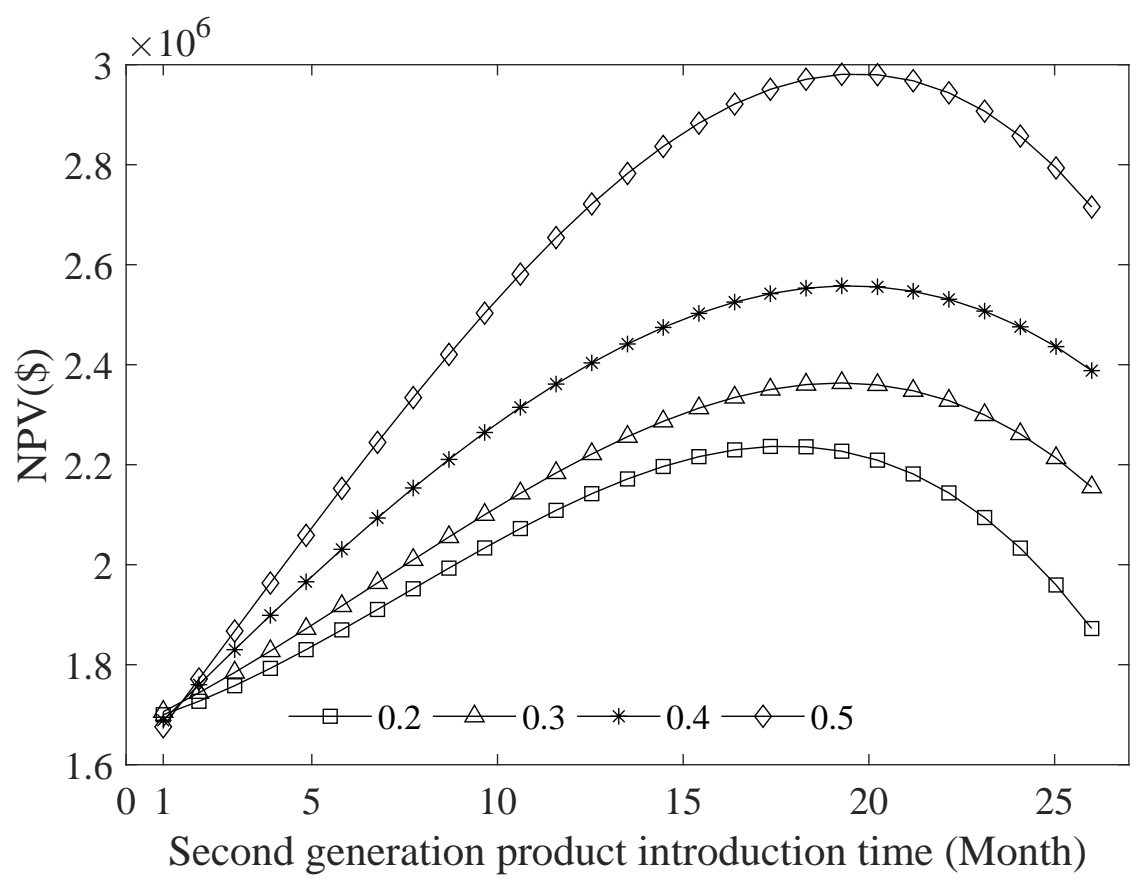

Figure 4.15: Second-generation PIT for different volatilities of product $A$ in growth regime

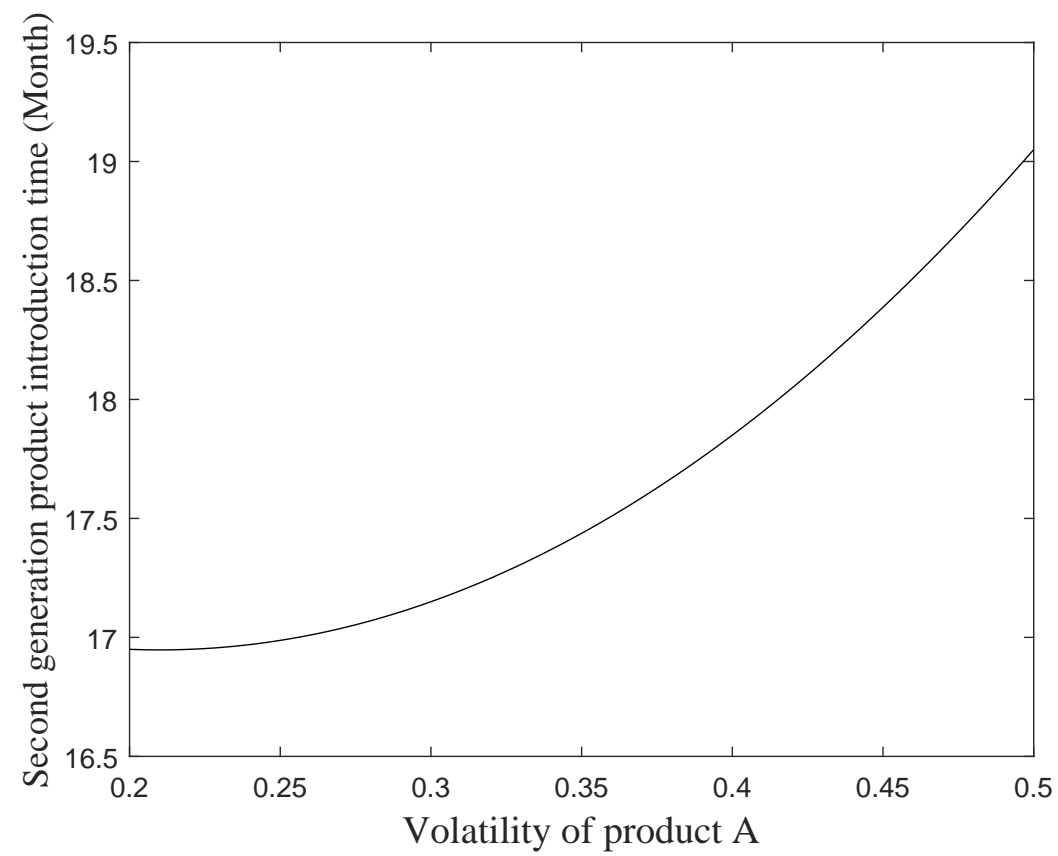

Figure 4.16: Second-generation PIT for different volatilities of product $A$ in growth regime

In Figure $4.17, t=0$ shows the result of Scenario 3 , and $t=1$ to $t=17$ shows the results of Scenario 2. Figure 4.17 shows that NPV is less than $\$ 10$ million when the second PIT is 
zero. NPV reaches a peak value of $\$ 59$ million when the volatility of product $A$ is 0.5 and the second PIT is delayed to 13 months. In Scenario 2, Figure 4.17 shows that when the volatility is 0.1 , the second PIT is estimated at month 11 . For example, the maximum NPV is obtained at month 11. Demand variation of product $A$ increases as the volatility increases from 0.1 to 0.5 . This is so that product $A$ lasts longer in the market. Figure 4.18 shows that as the volatility of product $A$ increases from 0.1 to 0.5 , second-generation PIT increases from month 11 to month 13 . This highlights that product $A$ remains in the market for 2 more months.

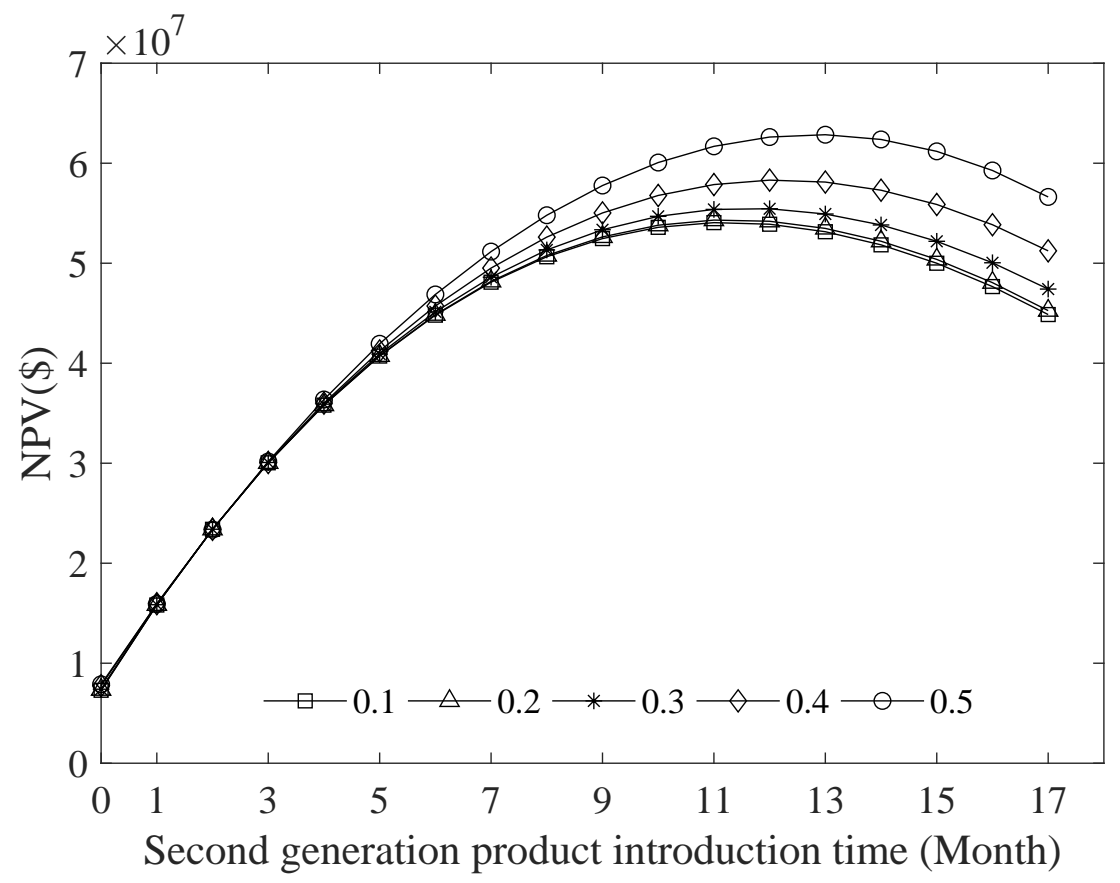

Figure 4.17: Second-generation PIT for different volatilities of product $A$ in growth regime 


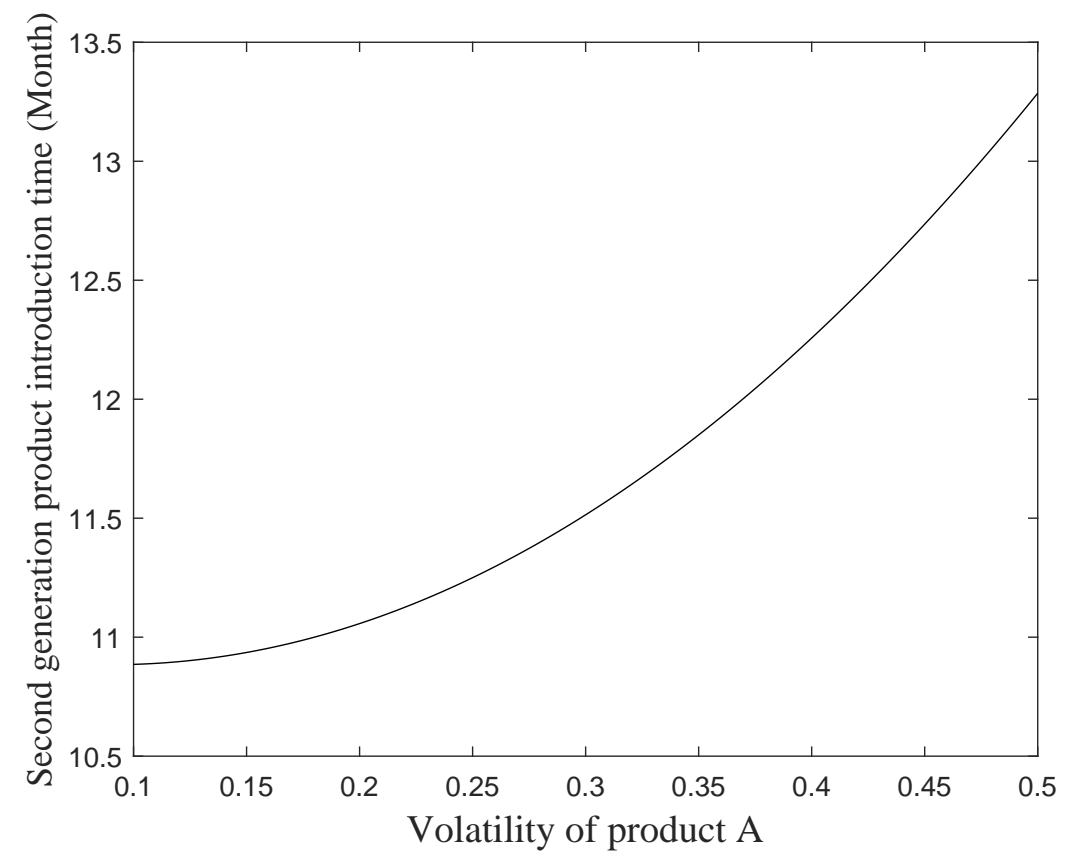

Figure 4.18: Second-generation PIT for different volatilities of product $A$ in growth regime 


\subsection{Summary}

Using the lattice method, this chapter investigated the best second-generation PIT. Dynamic programming method maximizes the NPV over a given period of time. Changing the drift rate, volatility, initial demand, and unit cost will affect how the NPV is analyzed. The sensitive analyses are investigated when product $A$ is in the growth regime. Sensitive analyses are investigated in three different scenarios as described in Section 3.2. Demand of product

$A$ increases as the drift rate, volatility, and initial demand of product $\mathrm{A}$ increases. As a result, the second-generation product introduction needs to be delayed. While the unit cost of product $A$ increases, the NPV lowers, and the second-generation PIT shifts to the left. This means the second-generation product needs to be introduced earlier. 


\section{Chapter 5}

\section{The Valuation of Product}

\section{Introduction Strategies Considering}

\section{PLC and Pricing}

\section{$5.1 \quad$ Introduction}

Price is an important element in a competitive market. Pricing is a challenging task for any company and pricing strategies need to change as the product life cycle of a product progresses. Market-skimming and market-penetration are two pricing strategies for companies. Market-skimming uses a high initial price and is effective for developing markets. On the

other hand, market-penetration uses a low price initially to capture a large market share (Mahajan and Muller, 1991). Demand and cost are two factors that affect a company's pricing strategy. Pricing strategy is more crucial when there are two products from the same family in one company. Pricing the first-generation product will affect second-generation product pricing and conversely, pricing the second-generation product will affect the first-generation product pricing. The relationship between the demand for the two products makes the pricing strategy complicated. There is a research that has studied the impact of price on the diffusion curve. It is crucial that the manager knows how a change in the price will affect 
the demand. Bass et al. (1994) proposed a model that captures the effect of the price on the diffusion process. Bass (1969), Robinson and Lakhani (1975), and Kalish (1983) have studied optimal pricing policy. A decision-making framework is proposed in this research in order to find the optimal product introduction time while incorporating pricing. As the product demand changes through its PLC, having a flexible production capacity is essential for maximizing NPV over the cycle. Expansion, contraction, and switching are three types of flexible production capacity that are considered in this research. An overview of the second phase of research is shown in Figure 5.1.

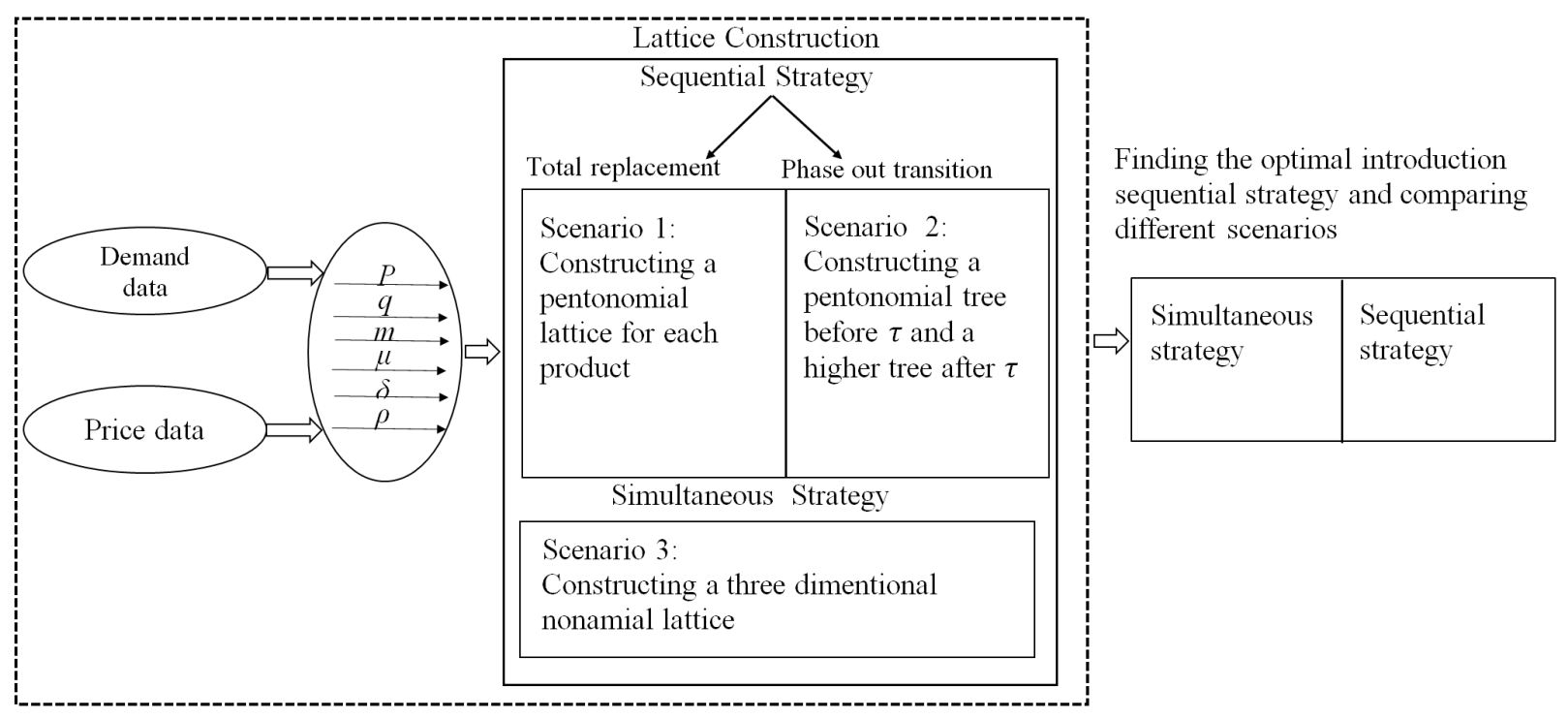

Figure 5.1: Overall framework of methodology

In the second model, estimating PLC parameters with the consideration of price effect on the diffusion curve is proposed. Each product regime is defined by a GBM. GBM is a continuous stochastic process in which the logarithm of the randomly varying quantity follows a Brownian motion with drift. Lattice is an approach to value a real asset and deals with early exercise; it is also used for discrete-time modelling. 


\subsection{Model development}

In this section, the steps to propose the second model are described. In the first step, Bass parameters (innovation coefficient, $p$, imitation coefficient, $q$, and market size, $m$ ) are estimated using the method explained in Section 3.2.1. The stochastic demand parameters (i.e., drift rate, volatility, and correlation) are then calculated using the diffusion adoption function of each product (Jiang and Jain, 2012a). More details of estimating the stochastic demand parameters are described in Section 5.3. Finally, the lattice approach is used to model product demands; detailed steps are described in section 4.4. A dynamic programming approach is used to find the maximum NPV over a given period of time.

\subsection{Estimating the stochastic demand parameters}

The demand probability distribution function for each product is calculated using the following diffusing adoption function (Jiang and Jain, 2012a), and Bass parameters from the following equations:

$$
F_{i}(t)=\left\{\begin{array}{ll}
0 & \text { if } t<0 \\
\frac{1-e^{-\left(p_{i}+q_{i}\right) X_{i}(t)}}{\left(q_{i} / p_{i}\right) e^{-\left(p_{i}+q_{i}\right) X_{i}(t)}+1} & \text { if } t \geq 0
\end{array},\right.
$$

then

$$
f_{i}(t)=\frac{\left(p_{i}+q_{i}\right)^{2}}{p_{i}} x_{i}(t) \frac{e^{-\left(p_{i}+q_{i}\right) X_{i}(t)}}{\left[\left(q_{i} / p_{i}\right) e^{-\left(p_{i}+q_{i}\right) X_{i}(t)}+1\right]^{2}},
$$

and sales at $t$

$$
S_{i}(t)=m_{i} \frac{\left(p_{i}+q_{i}\right)^{2}}{p_{i}} x_{i}(t) \frac{e^{-\left(p_{i}+q_{i}\right) X_{i}(t)}}{\left[\left(q_{i} / p_{i}\right) e^{-\left(p_{i}+q_{i}\right) X_{i}(t)}+1\right]^{2}} .
$$

Where $p_{i}$ and $q_{i}$ are the coefficient of innovation and imitation, respectively for product $\mathrm{i}$ $(i \in\{A, B\})$ and $X_{i}(t)$ is the price function.

$$
X_{i}(t)=t+\beta_{1_{i}} \ln \left[\frac{P r_{i}(t)}{P r_{i}(0)}\right]
$$




$$
x_{i}(t)=1+\beta_{1_{i}}\left[\frac{P r_{i}^{\prime}(t)}{P r_{i}(t)}\right] .
$$

where $x_{i}(t)$ is the current marketing effect and $\operatorname{Pr}_{i}^{\prime}(t)$ is the rate of change in the price. Furthermore, $\operatorname{Pr}_{i}(t)$ and $\operatorname{Pr}_{i}(0)$ are the prices of each product at time $t$ and zero, respectively. $\beta_{1_{i}}$ is the diffusion price parameter and represents the effectiveness of price over time.

The sales price and demand data for Dram $4 \mathrm{~K}$ and $16 \mathrm{~K}$ is used for analysis (Norton and Bass, 1987). Equation (5.3) is used to estimate the Bass parameters for products $A$ and $B$. Table 5.1 shows the estimated Bass parameters for products $A$ and $B$.

Table 5.1: Estimated Bass parameters for products $A$ and $B$

\begin{tabular}{|l||l|l|}
\hline Bass Parameters & Product $A$ & Product $B$ \\
\hline$p$ & 0.00897 & 0.00512 \\
$q$ & 0.921 & 0.962 \\
$m$ & 317 & 1303 \\
$\beta_{1}$ & -0.187 & 0.385 \\
\hline
\end{tabular}

The following steps describe how the stochastic demand parameters are calculated. There are two products $A$ and $B$ and each product could be in the growth or the decaying regime. Each product regime is defined by a GBM as below:

$$
\theta^{A}(t)=\text { Demand of product } A=\left\{\begin{array}{cc}
\theta_{g}^{A} & \text { if } t \leq t_{A} \\
\theta_{d}^{A} & \text { if } t>t_{A}
\end{array}\right.
$$

similarly, for product $B$

$$
\theta^{B}(t)=\text { Demand of product } B=\left\{\begin{array}{ccc}
\theta_{g}^{B} & \text { if } t \leq t_{B} \\
\theta_{d}^{B} & \text { if } t>t_{B}
\end{array}\right.
$$

The switching times for products $A$ and $B$ from growth to decay regime are denoted by $t_{A}$ and $t_{B}$, respectively. When the price only affects drift rate, we have:

$$
\frac{\mathrm{d} \theta_{g}^{A}(t)}{\theta_{g}^{A}(t)}=\left(\mu_{g}^{A}+X^{\prime}(t)\right) \mathrm{d} t+\sigma_{g}^{A} \mathrm{~d} W_{t}^{A, g}, \mu_{g}^{A}>0
$$




$$
\begin{aligned}
& \frac{\mathrm{d} \theta_{d}^{A}(t)}{\theta_{d}^{A}(t)}=\left(\mu_{d}^{A}+X^{\prime}(t)\right) \mathrm{d} t+\sigma_{d}^{A} \mathrm{~d} W_{t}^{A, d}, \mu_{d}^{A}<0, \\
& \frac{\mathrm{d} \theta_{g}^{B}(t)}{\theta_{g}^{B}(t)}=\left(\mu_{g}^{B}+X^{\prime}(t)\right) \mathrm{d} t+\sigma_{g}^{B} \mathrm{~d} W_{t}^{B, g}, \mu_{g}^{B}>0, \\
& \frac{\mathrm{d} \theta_{d}^{B}(t)}{\theta_{d}^{B}(t)}=\left(\mu_{d}^{B}+X^{\prime}(t)\right) \mathrm{d} t+\sigma_{d}^{A} \mathrm{~d} W_{t}^{B, d}, \mu_{d}^{B}<0 .
\end{aligned}
$$

When the price affects both drift rate and volatility, we have (see Appendix C for details)

$$
\begin{aligned}
& \frac{\mathrm{d} \theta_{g}^{A}(t)}{\theta_{g}^{A}(t)}=\left[\mu_{g}^{A} X(t)+X^{\prime}(t)+\frac{X^{2}(t)}{2} \sigma_{g}^{2 A}\right] d t+X(t) \sigma_{g}^{A} \mathrm{~d} W_{t}^{A, g}, \mu_{g}^{A}>0, \\
& \frac{\mathrm{d} \theta_{d}^{A}(t)}{\theta_{d}^{A}(t)}=\left[\mu_{d}^{A} X(t)+X^{\prime}(t)+\frac{X^{2}(t)}{2} \sigma_{d}^{2 A}\right] d t+X(t) \sigma_{d}^{A} \mathrm{~d} W_{t}^{A, d}, \mu_{d}^{A}<0, \\
& \frac{\mathrm{d} \theta_{g}^{B}(t)}{\theta_{g}^{B}(t)}=\left[\mu_{g}^{B} X(t)+X^{\prime}(t)+\frac{X^{2}(t)}{2} \sigma_{g}^{2 B}\right] d t+X(t) \sigma_{g}^{B} \mathrm{~d} W_{t}^{B, g}, \mu_{g}^{B}>0, \\
& \frac{\mathrm{d} \theta_{d}^{B}(t)}{\theta_{d}^{B}(t)}=\left[\mu_{d}^{B} X(t)+X^{\prime}(t)+\frac{X^{2}(t)}{2} \sigma_{d}^{2 B}\right] d t+X(t) \sigma_{d}^{B} \mathrm{~d} W_{t}^{B, d}, \mu_{d}^{B}<0 .
\end{aligned}
$$

For identifying the characteristics of the product life cycle, historical data is used. By using the historical data for each product, the product regimes are identified. The following steps describe how the demand parameters are estimated. As each product is represented by a GBM, the growth regime of the product $\mathrm{A}$ is defined as

$$
U_{\mathrm{g}}^{A}(t)=\operatorname{Ln}\left[\frac{\theta_{\mathrm{g}}^{A}(t)}{\theta_{\mathrm{g}}^{A}(t-1)}\right],
$$

and average of $U_{\mathrm{g}}^{A}(t)$ is

$$
\bar{U}_{\mathrm{g}}^{A}(t)=(1 / n) \sum_{t=1}^{n} U_{\mathrm{g}}^{A}(t) .
$$

Then, drift rate, $\mu_{g}^{A}$, can be estimated as

$$
\bar{U}_{g}^{A}(t) / h
$$

Similarly, we can define $\mu_{d}^{A}, \mu_{g}^{B}$, and $\mu_{d}^{B} . h$ as the time in the year between two consecutive 
observations. Next, $\sigma_{\mathrm{g}}^{A}$, the volatility of product $A$ in the growth regime can be found in Ross (1999) and Hull (2009) as

$$
\sigma_{\mathrm{g}}^{A} / \sqrt{h}
$$

where

$$
\sigma_{\mathrm{g}}^{A}=\sqrt{(1 / n-1) \sum_{\mathrm{t}=1}^{n}\left(U_{\mathrm{g}}^{A}(t)-\bar{U}_{\mathrm{g}}^{A}(t)\right)^{2}} .
$$

Estimation of other regime parameters can be calculated as above. Similarly, we can define $\sigma_{d}^{A}, \sigma_{g}^{B}$, and $\sigma_{d}^{B}$. The correlation between demands in a combined regime $\left(A_{g}, B_{g}\right)$ can be calculated as

$$
\rho_{g g}=\left[\sum_{\mathrm{t}=1}^{n}\left(U_{\mathrm{g}}^{A}(t)-\bar{U}_{\mathrm{g}}^{A}(t)\right) \cdot\left(U_{\mathrm{g}}^{B}(t)-\bar{U}_{\mathrm{g}}^{B}(t)\right)\right] /(n-1) \sigma_{\mathrm{g}}^{A} \sigma_{\mathrm{g}}^{B}
$$

similarly, for other combined regimes $\left(A_{d}, B_{g}\right),\left(A_{g}, B_{d}\right)$, and $\left(A_{d}, B_{d}\right)$, we have $\rho_{d g}, \rho_{g d}$, and $\rho_{d d}$, respectively.

$$
\begin{aligned}
& \rho_{d g}=\left[\sum_{\mathrm{t}=1}^{n}\left(U_{\mathrm{d}}^{A}(t)-\bar{U}_{\mathrm{d}}^{A}(t)\right) \cdot\left(U_{\mathrm{g}}^{B}(t)-\bar{U}_{\mathrm{g}}^{B}(t)\right)\right] /(n-1) \sigma_{\mathrm{d}}^{A} \sigma_{\mathrm{g}}^{B}, \\
& \rho_{g d}=\left[\sum_{\mathrm{t}=1}^{n}\left(U_{\mathrm{g}}^{A}(t)-\bar{U}_{\mathrm{g}}^{A}(t)\right) \cdot\left(U_{\mathrm{d}}^{B}(t)-\bar{U}_{\mathrm{d}}^{B}(t)\right)\right] /(n-1) \sigma_{\mathrm{g}}^{A} \sigma_{\mathrm{d}}^{B}, \\
& \rho_{d d}=\left[\sum_{\mathrm{t}=1}^{n}\left(U_{\mathrm{d}}^{A}(t)-\bar{U}_{\mathrm{d}}^{A}(t)\right) \cdot\left(U_{\mathrm{d}}^{B}(t)-\bar{U}_{\mathrm{d}}^{B}(t)\right)\right] /(n-1) \sigma_{\mathrm{d}}^{A} \sigma_{\mathrm{d}}^{B} .
\end{aligned}
$$

Demand parameters for products $A$ and $B$ are estimated using estimated Bass parameters from Equation (5.3) and the method is explained above. Sales for the first- and secondgenerations products are calculated using Equations (5.1 and 5.2) and following equations.

$$
S_{A}(t)= \begin{cases}m_{A} f_{A}(t) & \text { if } t<\tau \\ m_{A} f_{A}(t)\left[1-F_{B}(t-\tau)\right], & \text { if } t \geq \tau,\end{cases}
$$

where $\tau$ is the second-generation product introduction time. For the second-generation 
product we have

$$
S_{B}(t)=\left[m_{B}+m_{A} F_{A}(t)\right] f_{B}(t-\tau)+m_{A} f_{A}(t) F_{B}(t-\tau), \text { if } t \geq \tau .
$$

Table 5.2 shows the estimated drift rate and volatility for product $A$.

Table 5.2: Estimated demand parameters for product $A$

\begin{tabular}{|l||l|l|}
\hline Parameters & Growth regime & Decay regime \\
\hline Drift rate & 0.0651599 & -0.0374979 \\
Volatility & 0.0226732 & 0.0214119 \\
\hline
\end{tabular}

Table 5.3 shows the estimated drift rate and volatility for product $B$.

Table 5.3: Estimated demand parameters for product $B$

\begin{tabular}{|l||l|l|}
\hline Parameters & Growth regime & Decay regime \\
\hline Drift rate & 0.0133187 & -0.0299128 \\
Volatility & 0.0059599 & 0.0466865 \\
\hline
\end{tabular}

Table 5.4 shows the estimated correlation of combined regimes $\left(A_{g}, B_{g}\right),\left(A_{d}, B_{g}\right),\left(A_{g}, B_{d}\right)$, and $\left(A_{d}, B_{d}\right)$.

Table 5.4: Estimated correlated demand for combined regimes

\begin{tabular}{|l|l|l|l|}
\hline$\rho_{g g}$ & $\rho_{g d}$ & $\rho_{d g}$ & $\rho_{d d}$ \\
\hline 0.850465 & -0.184054 & -0.400598 & 0.505163 \\
\hline
\end{tabular}

\subsection{Lattice approach}

This section explains the lattice approach that is used in the second phase. Step 1 of constructing the lattice is described below. However, Steps 2 to 6 are the same as the steps 
explained in Section 4.4 .

\subsubsection{When price changes the drift rate}

In the discrete time model, changes in price will affect both drift rate and volatility that are important parameters in the PLC. This chapter addressees such effects while two regimes are considered for each product where each regime is defined by a GBM. By looking at the drift rate, the average increase or decrease on price over time can be captured. The following section investigates effects of price change only on drift rate. Let $F=\theta e^{\mu h+X(t)}$, where $X(t)$, price function, is a function of time.

Using Ito's Lemma (see Appendix A for details):

$$
d F=\left[\mu+X^{\prime}(t)\right] F d t+\sigma F d z
$$

Correlated demand for products $A$ and $B$ in combined regime $\left(A_{g}, B_{g}\right)$

$$
\begin{aligned}
& d \theta_{g}^{A}=\left[\mu_{g}^{A}+X^{\prime}(t)\right] \theta_{g}^{A} d t+\sigma_{g}^{A} \theta_{g}^{A} d Z_{g}^{A}, \\
& d \theta_{g}^{B}=\left[\mu_{g}^{B}+X^{\prime}(t)\right] \theta_{g}^{B} d t+\sigma_{g}^{B} \theta_{g}^{B} d Z_{g}^{B} .
\end{aligned}
$$

Using Ito's Lemma and Appendix D, Equations (5.26-5.27) are transformed to the following equations.

$$
\begin{aligned}
& d \ln \theta_{g}^{A}=\left[\mu_{g}^{A}-\frac{1}{2} \sigma_{g}^{2 A}+X^{\prime}(t)\right] d t+\sigma_{g}^{A} d z_{g}^{A}, \\
& d \ln \theta_{g}^{B}=\left[\mu_{g}^{B}-\frac{1}{2} \sigma_{g}^{2}{ }_{g}^{B}+X^{\prime}(t)\right] d t+\sigma_{g}^{B} d z_{g}^{B} .
\end{aligned}
$$

According to the above two equations and using method in Appendix D, eight uncorrelated 
processes are obtained.

$$
\begin{aligned}
d \Psi_{1} & =\left(\left[\mu_{g}^{A}-\frac{1}{2} \sigma_{g}^{2^{A}}+X^{\prime}(t)\right] \sigma_{g}^{B}+\left[\mu_{g}^{B}-\frac{1}{2}{\sigma^{2}}_{g}^{B}+X^{\prime}(t)\right] \sigma_{g}^{A}\right) d t \\
& +\sigma_{g}^{A} \sigma_{g}^{B} \sqrt{2\left(1+\rho_{g g}\right)} d z \Psi_{1}, \\
d \Psi_{2} & =\left(\left[\mu_{g}^{A}-\frac{1}{2} \sigma_{g}^{2}+X^{\prime}(t)\right] \sigma_{g}^{B}-\left[\mu_{g}^{B}-\frac{1}{2} \sigma_{g}^{2^{B}}+X^{\prime}(t)\right] \sigma_{g}^{A}\right) d t \\
& +\sigma_{g}^{A} \sigma_{g}^{B} \sqrt{2\left(1-\rho_{g g}\right)} d z \Psi_{2} .
\end{aligned}
$$

Where $\left(\left[\mu_{g}^{A}-\frac{1}{2} \sigma_{g}^{2 A}+X^{\prime}(t)\right] \sigma_{g}^{B}+\left[\mu_{g}^{B}-\frac{1}{2} \sigma_{g}^{2 B}+X^{\prime}(t)\right] \sigma_{g}^{A}\right)$ and

$\left(\left[\mu_{g}^{A}-\frac{1}{2} \sigma_{g}^{2 A}+X^{\prime}(t)\right] \sigma_{g}^{B}-\left[\mu_{g}^{B}-\frac{1}{2} \sigma_{g}^{2 B}+X^{\prime}(t)\right] \sigma_{g}^{A}\right)$ are the instantaneous mean for combined regime $\left(A_{g}, B_{g}\right)$ in each group,

$\sigma_{g}^{A} \sigma_{g}^{B} \sqrt{2\left(1+\rho_{g g}\right)}$ and $\sigma_{g}^{A} \sigma_{g}^{B} \sqrt{2\left(1-\rho_{g g}\right)}$ are the instantaneous volatility for combined regime $\left(A_{g}, B_{g}\right)$ in each group, and $d z_{\Psi_{1}}$ and $d z_{\Psi_{2}}$ are the uncorrelated Wiener processes. Details of the components of the Equations (5.30-5.37) are listed in the Table 5.5.

The detail of steps 2 to 6 are explained in section 4.4 .

Similarly, for combined regime $\left(A_{d}, B_{g}\right)$

$$
\begin{aligned}
d \Psi_{3} & =\left(\left[\mu_{d}^{A}-\frac{1}{2} \sigma_{d}^{2 A}+X^{\prime}(t)\right] \sigma_{g}^{B}+\left[\mu_{g}^{B}-\frac{1}{2}{\sigma^{2}}_{g}^{B}+X^{\prime}(t)\right] \sigma_{d}^{A}\right) d t \\
& +\sigma_{d}^{A} \sigma_{g}^{B} \sqrt{2\left(1+\rho_{d g}\right)} d z \Psi_{3}, \\
d \Psi_{4} & =\left(\left[\mu_{d}^{A}-\frac{1}{2} \sigma_{d}^{2 A}+X^{\prime}(t)\right] \sigma_{g}^{B}-\left[\mu_{g}^{B}-\frac{1}{2} \sigma_{g}^{2^{B}}+X^{\prime}(t)\right] \sigma_{d}^{A}\right) d t \\
& +\sigma_{d}^{A} \sigma_{g}^{B} \sqrt{2\left(1-\rho_{d g}\right)} d z \Psi_{4} .
\end{aligned}
$$


Also for combined regime $\left(A_{g}, B_{d}\right)$

$$
\begin{aligned}
d \Psi_{5} & =\left(\left[\mu_{g}^{A}-\frac{1}{2} \sigma_{g}^{2 A}+X^{\prime}(t)\right] \sigma_{d}^{B}+\left[\mu_{d}^{B}-\frac{1}{2}{\sigma^{2}}_{d}^{B}+X^{\prime}(t)\right] \sigma_{g}^{A}\right) d t \\
& +\sigma_{g}^{A} \sigma_{d}^{B} \sqrt{2\left(1+\rho_{g d}\right)} d z \Psi_{5}, \\
d \Psi_{6} & =\left(\left[\mu_{g}^{A}-\frac{1}{2} \sigma_{g}^{2 A}+X^{\prime}(t)\right] \sigma_{d}^{B}-\left[\mu_{d}^{B}-\frac{1}{2} \sigma_{d}^{2 B}+X^{\prime}(t)\right] \sigma_{g}^{A}\right) d t \\
& +\sigma_{d}^{A} \sigma_{g}^{B} \sqrt{2\left(1-\rho_{g d}\right)} d z \Psi_{6} .
\end{aligned}
$$

For combined regime $\left(A_{d}, B_{d}\right)$ we have:

$$
\begin{aligned}
d \Psi_{7} & =\left(\left[\mu_{d}^{A}-\frac{1}{2}{\sigma^{2}}_{d}^{A}+X^{\prime}(t)\right] \sigma_{d}^{B}+\left[\mu_{d}^{B}-\frac{1}{2}{\sigma^{2}}_{d}^{B}+X^{\prime}(t)\right] \sigma_{d}^{A}\right) d t \\
& +\sigma_{d}^{A} \sigma_{d}^{B} \sqrt{2\left(1+\rho_{d d}\right)} d z \Psi_{7}, \\
d \Psi_{8} & =\left(\left[\mu_{d}^{A}-\frac{1}{2} \sigma_{d}^{2 A}+X^{\prime}(t)\right] \sigma_{d}^{B}-\left[\mu_{d}^{B}-\frac{1}{2} \sigma_{d}^{2 B}+X^{\prime}(t)\right] \sigma_{d}^{A}\right) d t \\
& +\sigma_{d}^{A} \sigma_{d}^{B} \sqrt{2\left(1-\rho_{d d}\right)} d z \Psi_{8} .
\end{aligned}
$$


Table 5.5: Instantaneous mean and volatility of uncorrelated processes

\begin{tabular}{|c|c|c|}
\hline Process & Instantaneous Mean & $\begin{array}{l}\text { Instantaneous } \\
\text { Volatility }\end{array}$ \\
\hline $\begin{array}{l}\left(A_{g}, B_{g}\right)_{1} \\
\left(A_{g}, B_{g}\right)_{2}\end{array}$ & $\begin{array}{l}{\left[\mu_{g}^{A}-\frac{1}{2} \sigma_{g}^{2 A}+X^{\prime}(t)\right] \sigma_{g}^{B}+\left[\mu_{g}^{B}-\frac{1}{2} \sigma_{g}^{2 B}+X^{\prime}(t)\right] \sigma_{g}^{A}} \\
{\left[\mu_{g}^{A}-\frac{1}{2} \sigma_{g}^{2 A}+X^{\prime}(t)\right] \sigma_{g}^{B}-\left[\mu_{g}^{B}-\frac{1}{2} \sigma_{g}^{2 B}+X^{\prime}(t)\right] \sigma_{g}^{A}} \\
\end{array}$ & $\begin{array}{l}\sigma_{g}^{A} \sigma_{g}^{B} \sqrt{2\left(1+\rho_{g g}\right)} \\
\sigma_{g}^{A} \sigma_{g}^{B} \sqrt{2\left(1-\rho_{g g}\right)} \\
\end{array}$ \\
\hline $\begin{array}{l}\left(A_{d}, B_{g}\right)_{1} \\
\left(A_{d}, B_{g}\right)_{2}\end{array}$ & $\begin{array}{l}{\left[\mu_{d}^{A}-\frac{1}{2} \sigma_{d}^{2 A}+X^{\prime}(t)\right] \sigma_{g}^{B}+\left[\mu_{g}^{B}-\frac{1}{2} \sigma_{g}^{2 B}+X^{\prime}(t)\right] \sigma_{d}^{A}} \\
{\left[\mu_{d}^{A}-\frac{1}{2} \sigma_{d}^{2 A}+X^{\prime}(t)\right] \sigma_{g}^{B}-\left[\mu_{g}^{B}-\frac{1}{2} \sigma_{g}^{2 B}+X^{\prime}(t)\right] \sigma_{d}^{A}}\end{array}$ & $\begin{array}{l}\sigma_{d}^{A} \sigma_{g}^{B} \sqrt{2\left(1+\rho_{d g}\right)} \\
\sigma_{d}^{A} \sigma_{g}^{B} \sqrt{2\left(1-\rho_{d g}\right)} \\
\end{array}$ \\
\hline $\begin{array}{l}\left(A_{g}, B_{d}\right)_{1} \\
\left(A_{g}, B_{d}\right)_{2}\end{array}$ & $\begin{array}{l}{\left[\mu_{g}^{A}-\frac{1}{2} \sigma_{g}^{{ }^{A}}+X^{\prime}(t)\right] \sigma_{d}^{B}+\left[\mu_{d}^{B}-\frac{1}{2} \sigma_{d}^{2 B}+X^{\prime}(t)\right] \sigma_{g}^{A}} \\
{\left[\mu_{g}^{A}-\frac{1}{2} \sigma_{g}^{2^{A}}+X^{\prime}(t)\right] \sigma_{d}^{B}-\left[\mu_{d}^{B}-\frac{1}{2} \sigma_{d}^{2 B}+X^{\prime}(t)\right] \sigma_{g}^{A}} \\
\end{array}$ & $\begin{array}{c}\sigma_{g}^{A} \sigma_{d}^{B} \sqrt{2\left(1+\rho_{g d}\right)} \\
\sigma_{g}^{A} \sigma_{d}^{B} \sqrt{2\left(1-\rho_{g d}\right)} \\
\end{array}$ \\
\hline $\begin{array}{l}\left(A_{d}, B_{d}\right)_{1} \\
\left(A_{d}, B_{d}\right)_{2}\end{array}$ & $\begin{array}{l}{\left[\mu_{d}^{A}-\frac{1}{2} \sigma_{d}^{2 A}+X^{\prime}(t)\right] \sigma_{d}^{B}+\left[\mu_{d}^{B}-\frac{1}{2} \sigma_{d}^{2 B}+X^{\prime}(t)\right] \sigma_{d}^{A}} \\
{\left[\mu_{d}^{A}-\frac{1}{2} \sigma_{d}^{2 A}+X^{\prime}(t)\right] \sigma_{d}^{B}-\left[\mu_{d}^{B}-\frac{1}{2} \sigma_{d}^{2 B}+X^{\prime}(t)\right] \sigma_{d}^{A}}\end{array}$ & $\begin{array}{l}\sigma_{d}^{A} \sigma_{d}^{B} \sqrt{2\left(1+\rho_{d d}\right)} \\
\sigma_{d}^{A} \sigma_{d}^{B} \sqrt{2\left(1-\rho_{d d}\right)}\end{array}$ \\
\hline
\end{tabular}

\subsubsection{When price changes both volatility and drift rate}

In the real world economy, there is always a risk. Fluctuation of market price is an important parameter that has to be considered to capture the market dynamics over time. Following is a model developed to incorporate such effect. In this section, effect of changes in price on both drift rate and volatility will be investigated. Accordingly, when price changes, the formula becomes as follow: $F=e^{\mu h+X(t) \theta}$, where $X(t)$, price function, is a function of time. Using Ito's Lemma (see Appendix B for details):

$$
d F=\left[\mu X(t)+X^{\prime}(t)+\frac{X^{2}(t)}{2} \sigma^{2}\right] F d t+X(t) \sigma F d z
$$

Correlated demand for products $A$ and $B$ in combined regime $\left(A_{g}, B_{g}\right)$

$$
d \theta_{g}^{A}=\left[\mu_{g}^{A} X(t)+X^{\prime}(t)+\frac{X^{2}(t)}{2} \sigma_{g}^{2 A}\right] \theta_{g}^{A} d t+X(t) \sigma_{g}^{A} \theta_{g}^{A} d Z_{g}^{A},
$$




$$
d \theta_{g}^{B}=\left[\mu_{g}^{B} X(t)+X^{\prime}(t)+\frac{X^{2}(t)}{2} \sigma_{g}^{2 B}\right] \theta_{g}^{B} d t+X(t) \sigma_{g}^{B} \theta_{g}^{B} d Z_{g}^{B} .
$$

Using Ito's Lemma and Appendix D, Equations (5.39-5.40) can be rewritten as following equations.

$$
\begin{aligned}
& d \ln \theta_{g}^{A}=\left[\mu_{g}^{A} X(t)+X^{\prime}(t)\right] d t+\sigma_{g}^{A} X(t) d z_{g}^{A}, \\
& d \ln \theta_{g}^{B}=\left[\mu_{g}^{B} X(t)+X^{\prime}(t)\right] d t+\sigma_{g}^{B} X(t) d z_{g}^{B} .
\end{aligned}
$$

According to Equations (5.41 and 5.42) and using the method in Appendix D, eight uncorrelated processes are obtained. For combine regime $\left(A_{g}, B_{g}\right)$ we have:

$$
\begin{aligned}
d \Psi_{1} & =\left[\left(X(t)\left[\mu_{g}^{A} X(t)+X^{\prime}(t)\right] \sigma_{g}^{B}\right)\right]+\left[\left(X(t)\left[\mu_{g}^{B} X(t)+X^{\prime}(t)\right] \sigma_{g}^{A}\right)\right] d t \\
& +X^{2}(t) \sigma_{g}^{A} \sigma_{g}^{B} \sqrt{2\left(1+\rho_{g g}\right)} d z \Psi_{1}, \\
d \Psi_{2} & =\left[\left(X(t)\left[\mu_{g}^{A} X(t)+X^{\prime}(t)\right] \sigma_{g}^{B}\right)\right]-\left[\left(X(t)\left[\mu_{g}^{B} X(t)+X^{\prime}(t)\right] \sigma_{g}^{A}\right)\right] d t \\
& +X^{2}(t) \sigma_{g}^{A} \sigma_{g}^{B} \sqrt{2\left(1-\rho_{g g}\right)} d z \Psi_{2} .
\end{aligned}
$$

Where $\left[\left(X(t)\left[\mu_{g}^{A} X(t)+X^{\prime}(t)\right] \sigma_{g}^{B}\right)\right]+\left[\left(X(t)\left[\mu_{g}^{B} X(t)+X^{\prime}(t)\right] \sigma_{g}^{A}\right)\right]$ and $\left[\left(X(t)\left[\mu_{g}^{A} X(t)+X^{\prime}(t)\right] \sigma_{g}^{B}\right)\right]-\left[\left(X(t)\left[\mu_{g}^{B} X(t)+X^{\prime}(t)\right] \sigma_{g}^{A}\right)\right]$ are the instantaneous mean for combined regime $\left(A_{g}, B_{g}\right)$ in each group, $X^{2}(t) \sigma_{g}^{A} \sigma_{g}^{B} \sqrt{2\left(1+\rho_{g g}\right)}$ and $X^{2}(t) \sigma_{g}^{A} \sigma_{g}^{B} \sqrt{2\left(1-\rho_{g g}\right)}$ are the instantaneous volatility for combined regime $\left(A_{g}, B_{g}\right)$ in each group, and $d z_{\Psi_{1}}$ and $d z_{\Psi_{2}}$ are the uncorrelated Wiener processes. Details of the components of the Equations (5.43-5.50) are listed in the Table 5.6. Similarly, for combined regime $\left(A_{d}, B_{g}\right)$ 


$$
\begin{aligned}
d \Psi_{3} & =\left[\left(X(t)\left[\mu_{d}^{A} X(t)+X^{\prime}(t)\right] \sigma_{g}^{B}\right)\right]+\left[\left(X(t)\left[\mu_{g}^{B} X(t)+X^{\prime}(t)\right] \sigma_{d}^{A}\right)\right] d t \\
& +X^{2}(t) \sigma_{d}^{A} \sigma_{g}^{B} \sqrt{2\left(1+\rho_{d g}\right)} d z \Psi_{3}, \\
d \Psi_{4} & =\left[\left(X(t)\left[\mu_{d}^{A} X(t)+X^{\prime}(t)\right] \sigma_{g}^{B}\right)\right]-\left[\left(X(t)\left[\mu_{g}^{B} X(t)+X^{\prime}(t)\right] \sigma_{d}^{A}\right)\right] d t \\
& +X^{2}(t) \sigma_{d}^{A} \sigma_{g}^{B} \sqrt{2\left(1-\rho_{d g}\right)} d z \Psi_{4} .
\end{aligned}
$$

Also for combined regime $\left(A_{g}, B_{d}\right)$

$$
\begin{aligned}
d \Psi_{5} & =\left[\left(X(t)\left[\mu_{g}^{A} X(t)+X^{\prime}(t)\right] \sigma_{d}^{B}\right)\right]+\left[\left(X(t)\left[\mu_{d}^{B} X(t)+X^{\prime}(t)\right] \sigma_{g}^{A}\right)\right] d t \\
& +X^{2}(t) \sigma_{g}^{A} \sigma_{d}^{B} \sqrt{2\left(1+\rho_{g d}\right)} d z \Psi_{5}, \\
d \Psi_{6} & =\left[\left(X(t)\left[\mu_{g}^{A} X(t)+X^{\prime}(t)\right] \sigma_{d}^{B}\right)\right]-\left[\left(X(t)\left[\mu_{d}^{B} X(t)+X^{\prime}(t)\right] \sigma_{g}^{A}\right)\right] d t \\
& +X^{2}(t) \sigma_{g}^{A} \sigma_{d}^{B} \sqrt{2\left(1-\rho_{g d}\right)} d z \Psi_{6} .
\end{aligned}
$$

Also, for combined regime $\left(A_{d}, B_{d}\right)$

$$
\begin{aligned}
d \Psi_{7} & =\left[\left(X(t)\left[\mu_{d}^{A} X(t)+X^{\prime}(t)\right] \sigma_{d}^{B}\right)\right]+\left[\left(X(t)\left[\mu_{d}^{B} X(t)+X^{\prime}(t)\right] \sigma_{d}^{A}\right)\right] d t \\
& +X^{2}(t) \sigma_{d}^{A} \sigma_{d}^{B} \sqrt{2\left(1+\rho_{g g}\right)} d z \Psi_{7}, \\
d \Psi_{8} & =\left[\left(X(t)\left[\mu_{d}^{A} X(t)+X^{\prime}(t)\right] \sigma_{d}^{B}\right)\right]-\left[\left(X(t)\left[\mu_{d}^{B} X(t)+X^{\prime}(t)\right] \sigma_{d}^{A}\right)\right] d t \\
& +X^{2}(t) \sigma_{d}^{A} \sigma_{d}^{B} \sqrt{2\left(1-\rho_{g g}\right)} d z \Psi_{8} .
\end{aligned}
$$


Table 5.6: Instantaneous mean and volatility of uncorrelated processes

\begin{tabular}{|l||l|l|}
\hline Process & Instantaneous Mean & Instantaneous Volatility \\
\hline$\left(A_{g}, B_{g}\right)_{1}$ & $\left.X(t)\left[\mu_{g}^{A} X(t)+X^{\prime}(t)\right] \sigma_{g}^{B}\right]+X(t)\left[\mu_{g}^{B} X(t)+X^{\prime}(t)\right] \sigma_{g}^{A}$ & $X^{2}(t) \sigma_{g}^{A} \sigma_{g}^{B} \sqrt{2\left(1+\rho_{g g}\right)}$ \\
$\left(A_{g}, B_{g}\right)_{2}$ & $\left.X(t)\left[\mu_{g}^{A} X(t)+X^{\prime}(t)\right] \sigma_{g}^{B}\right]-X(t)\left[\mu_{g}^{B} X(t)+X^{\prime}(t)\right] \sigma_{g}^{A}$ & $X^{2}(t) \sigma_{g}^{A} \sigma_{g}^{B} \sqrt{2\left(1-\rho_{g g}\right)}$ \\
\hline \hline$\left(A_{d}, B_{g}\right)_{1}$ & $\left.X(t)\left[\mu_{d}^{A} X(t)+X^{\prime}(t)\right] \sigma_{g}^{B}\right]+X(t)\left[\mu_{g}^{B} X(t)+X^{\prime}(t)\right] \sigma_{d}^{A}$ & $X^{2}(t) \sigma_{d}^{A} \sigma_{g}^{B} \sqrt{2\left(1+\rho_{d g}\right)}$ \\
$\left(A_{d}, B_{g}\right)_{2}$ & $\left.X(t)\left[\mu_{d}^{A} X(t)+X^{\prime}(t)\right] \sigma_{g}^{B}\right]-X(t)\left[\mu_{g}^{B} X(t)+X^{\prime}(t)\right] \sigma_{d}^{A}$ & $X^{2}(t) \sigma_{d}^{A} \sigma_{g}^{B} \sqrt{2\left(1-\rho_{d g}\right)}$ \\
\hline \hline$\left(A_{g}, B_{d}\right)_{1}$ & $\left.X(t)\left[\mu_{g}^{A} X(t)+X^{\prime}(t)\right] \sigma_{d}^{B}\right]+X(t)\left[\mu_{d}^{B} X(t)+X^{\prime}(t)\right] \sigma_{g}^{A}$ & $X^{2}(t) \sigma_{g}^{A} \sigma_{d}^{B} \sqrt{2\left(1+\rho_{g d}\right)}$ \\
$\left(A_{g}, B_{d}\right)_{2}$ & $\left.X(t)\left[\mu_{g}^{A} X(t)+X^{\prime}(t)\right] \sigma_{d}^{B}\right]-X(t)\left[\mu_{d}^{B} X(t)+X^{\prime}(t)\right] \sigma_{g}^{A}$ & $X^{2}(t) \sigma_{g}^{A} \sigma_{d}^{B} \sqrt{2\left(1-\rho_{g d}\right)}$ \\
\hline \hline$\left(A_{d}, B_{d}\right)_{1}$ & $\left.X(t)\left[\mu_{d}^{A} X(t)+X^{\prime}(t)\right] \sigma_{d}^{B}\right]+X(t)\left[\mu_{d}^{B} X(t)+X^{\prime}(t)\right] \sigma_{d}^{A}$ & $X^{2}(t) \sigma_{d}^{A} \sigma_{d}^{B} \sqrt{2\left(1+\rho_{d d}\right)}$ \\
$\left(A_{d}, B_{d}\right)_{2}$ & $\left.X(t)\left[\mu_{d}^{A} X(t)+X^{\prime}(t)\right] \sigma_{d}^{B}\right]-X(t)\left[\mu_{d}^{B} X(t)+X^{\prime}(t)\right] \sigma_{d}^{A}$ & $X^{2}(t) \sigma_{d}^{A} \sigma_{d}^{B} \sqrt{2\left(1-\rho_{d d}\right)}$ \\
\hline
\end{tabular}

\subsection{Analysis and results}

We examined the three scenarios described in the methodology section with two different pricing policies considered in this research: decreasing and increasing-decreasing pricing. For the first-generation product, always decreasing pricing policy is considered, while for the second-generation product either decreasing or increasing-decreasing pricing policy is considered. In increasing-decreasing pricing policy, the change rate of the increasing part remains the same in the increasing and decreasing parts. A decreasing pricing policy attracts more price-sensitive consumers (Bolton, 1989). In contrast, an increasing-decreasing pricing policy draws consumers who are not as price sensitive as those of the decreasing pricing policy (Shankar and Krishnamurthi, 1996).

These three scenarios and two different pricing policies are examined in two different approaches. In the first approach, Approach (I), the price changes only the drift rate, and in the second approach, (II), the price changes both drift rate and volatility. The sales and demand data for Dram 4K and 16K are used for analysis (Norton and Bass, 1987).

Usually, large, established companies can produce a product at a lower cost compared 
with new companies due to the economics of scale. Thus, new companies may have to reduce their product price sale and determine how much profit they can make in a specific period of time. On the other hand, when a company has a significant competitive advantage, they price a product at a high level (Skimming strategy) to gain the maximum profit before other companies offer similar products.

\subsubsection{Total replacement}

In Scenario 1, total replacement and decreasing pricing policy, Figure 5.2 shows that as the pricing decreasing rate decreases from -0.3 to -0.7 , the second-generation PIT will be delayed. As the pricing decreasing rate decreases, the second-generation product lasts longer in the market. Figure 5.3 shows that as the second-generation product price decreases from -0.3 to -0.7 , demand for product $B$ increases, so the second-generation PIT increases from month 7 to month 9. Accordingly, product $B$ is expected to last longer in the market. This highlights that product $B$ remains in the market for 2 more months.

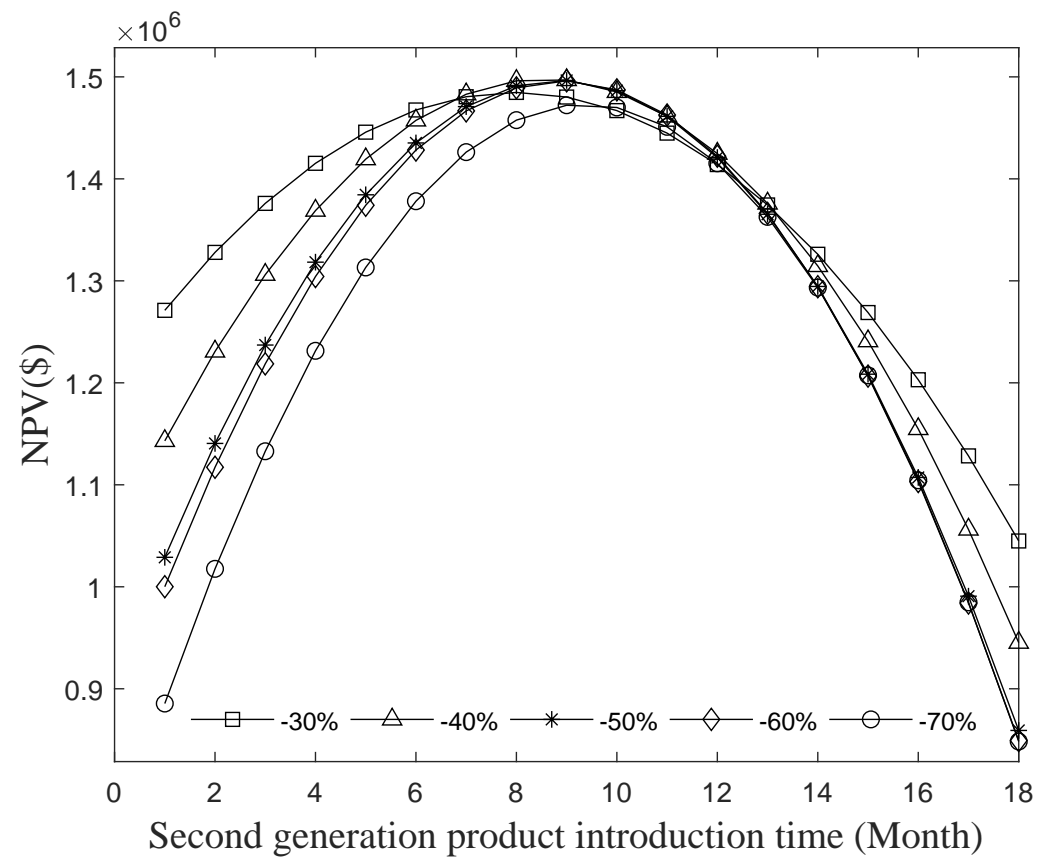

Figure 5.2: Second-generation PIT (Decreasing pricing policy-total replacement) 


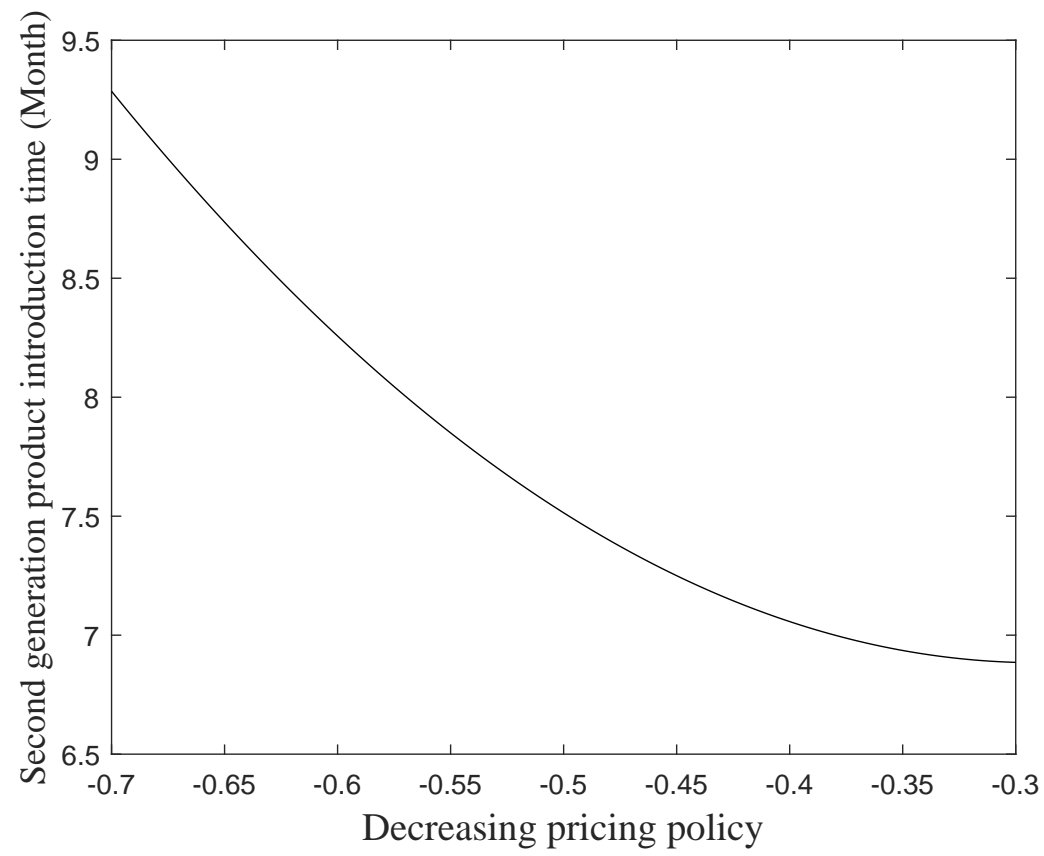

Figure 5.3: Second-generation PIT (Decreasing pricing policy-total replacement)

In Scenario 1, total replacement and increasing-decreasing pricing policy, Figure 5.4 shows the results when the increasing rate is 0.05 and the decreasing rate changes from -0.3 to -0.7 . When the decreasing pricing rate decreases from -0.3 to -0.7 , the second-generation PIT is delayed. As the pricing increasing-decreasing rate decreases, the second-generation product lasts longer in the market. Figure 5.5 shows the results when increasing-decreasing pricing policy decreases from -0.3 to -0.7 . As the second-generation product price decreases from -0.3 to -0.7 , the second-generation PIT increases from month 7 to month 10 because demand for product $B$ increases. This highlights that the product $B$ remains in the market for 3 more months due to the fact that product $B$ is expected to last longer in the market. 


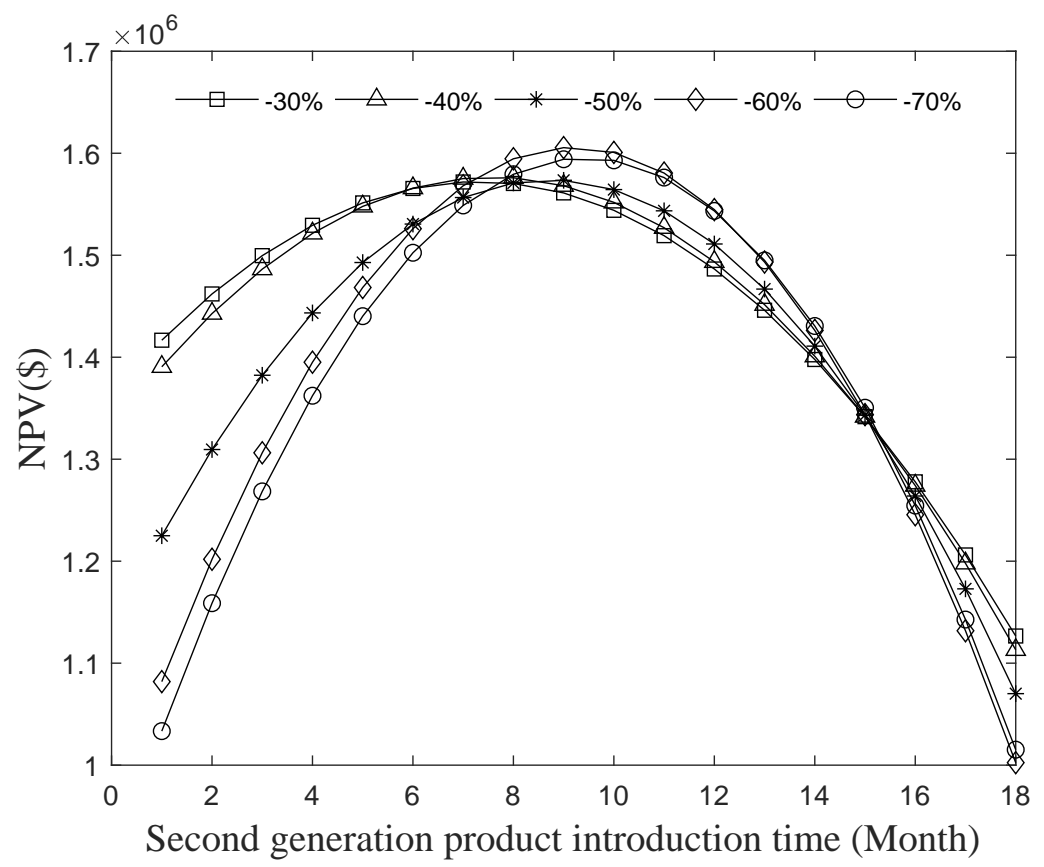

Figure 5.4: Second-generation PIT (Increasing-Decreasing pricing policy-total replacement)

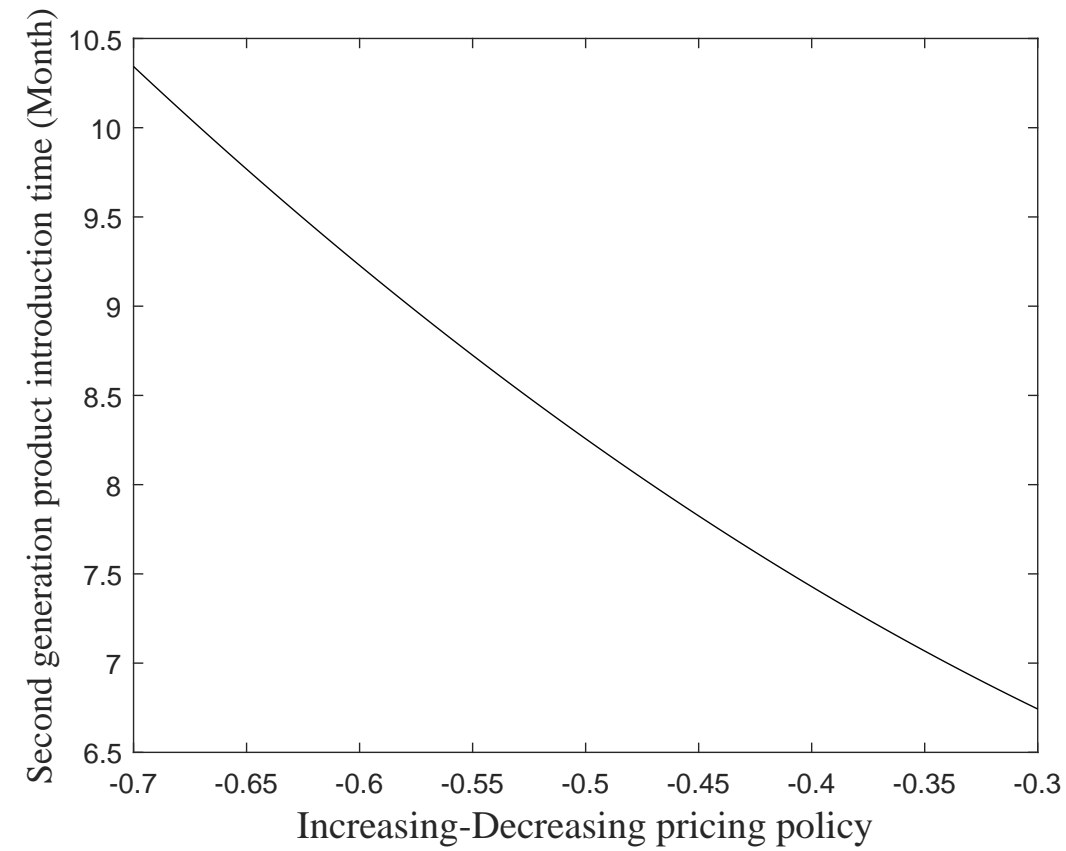

Figure 5.5: Second-generation PIT (Increasing-Decreasing pricing policy-total replacement) 


\subsubsection{Phase out transition}

In Figure 5.6, $t=0$ shows the result of Scenario 3, phase out transition-simultaneous strategy, and $t=1$ to $t=5$ show the results of Scenario 2, phase out transition-sequential strategy. Figure 5.6 shows the decreasing pricing policy when the price changes only the drift rate. When the pricing decreasing rate decreases from -0.3 to -0.7 , the second-generation PIT remains the same at month 2 , and the NPV reaches a peak value of $\$ 4.6$ million. In this scenario, the second-generation product has to be introduced as early as the first-generation PIT. Figure 5.7 shows the second-generation PIT remain at month 2. As the second-generation product uses decreasing pricing policy, demand for product $B$ increases. Therefore, the second-generation product has to be introduced as early as the first-generation PIT.

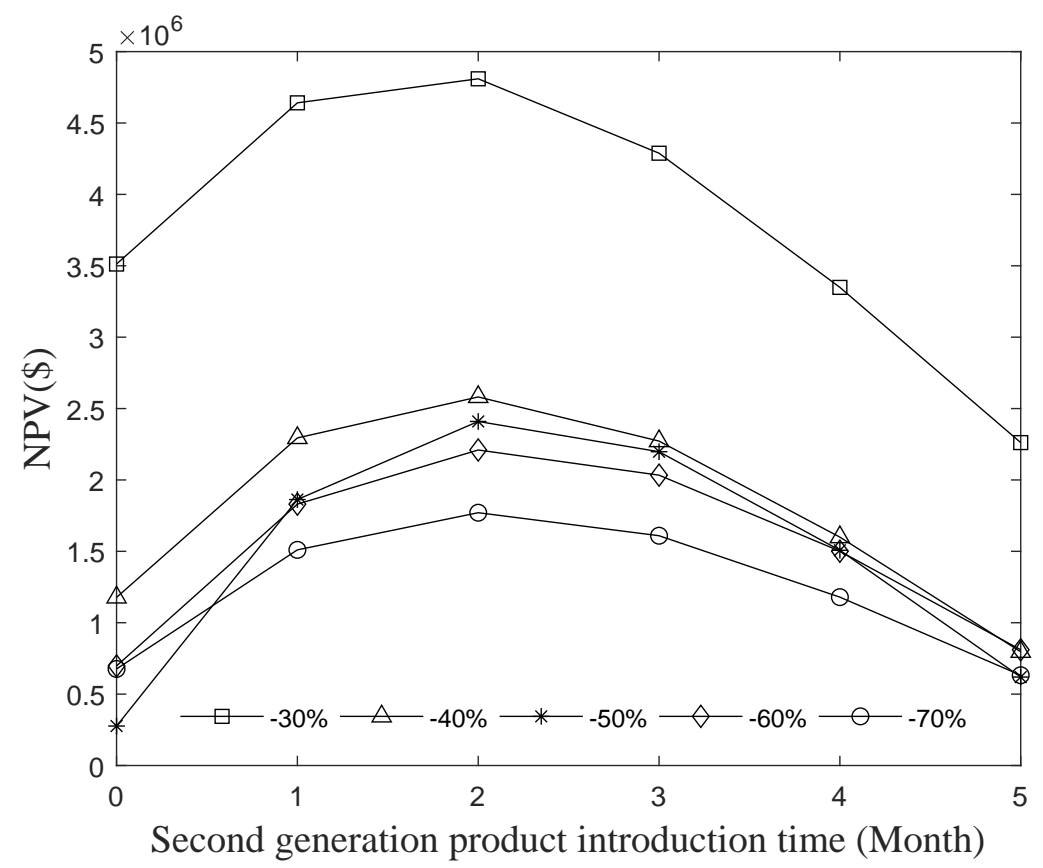

Figure 5.6: Second-generation PIT with Approach (I) (Decreasing pricing policy-phase out transition) 


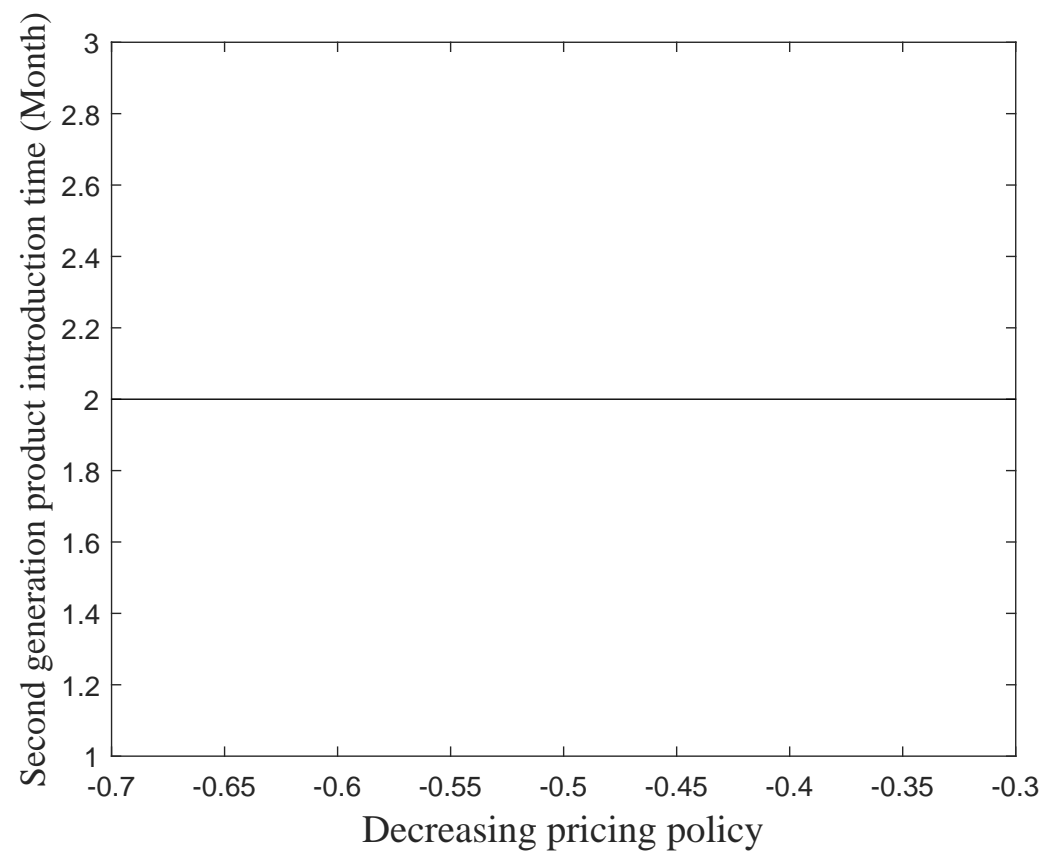

Figure 5.7: Second-generation PIT with Approach (I) (Decreasing pricing policy-phase out transition)

In Figure 5.8, $t=0$ shows the result of Scenario 3, phase out transition-simultaneous strategy, and $t=1$ to $t=9$ show the results of Scenario 2, phase out transition-sequential strategy. Figure 5.8 shows the decreasing pricing policy when the price changes both drift rate and volatility. When the pricing decreasing rate decreases from -0.3 to -0.7 , the secondgeneration PIT remains the same at month 3, and the NPV reaches a peak value of $\$ 35$ million. Figure 5.9 shows the second-generation PIT remain at month 3. It means in this scenario, the second-generation product has to be introduced as early as the first-generation PIT. In this scenario as decreasing pricing policy is used for the SGP, demand for the SGP increases. As a result, "as early as possible" policy is optimal for introducing the SGP. 


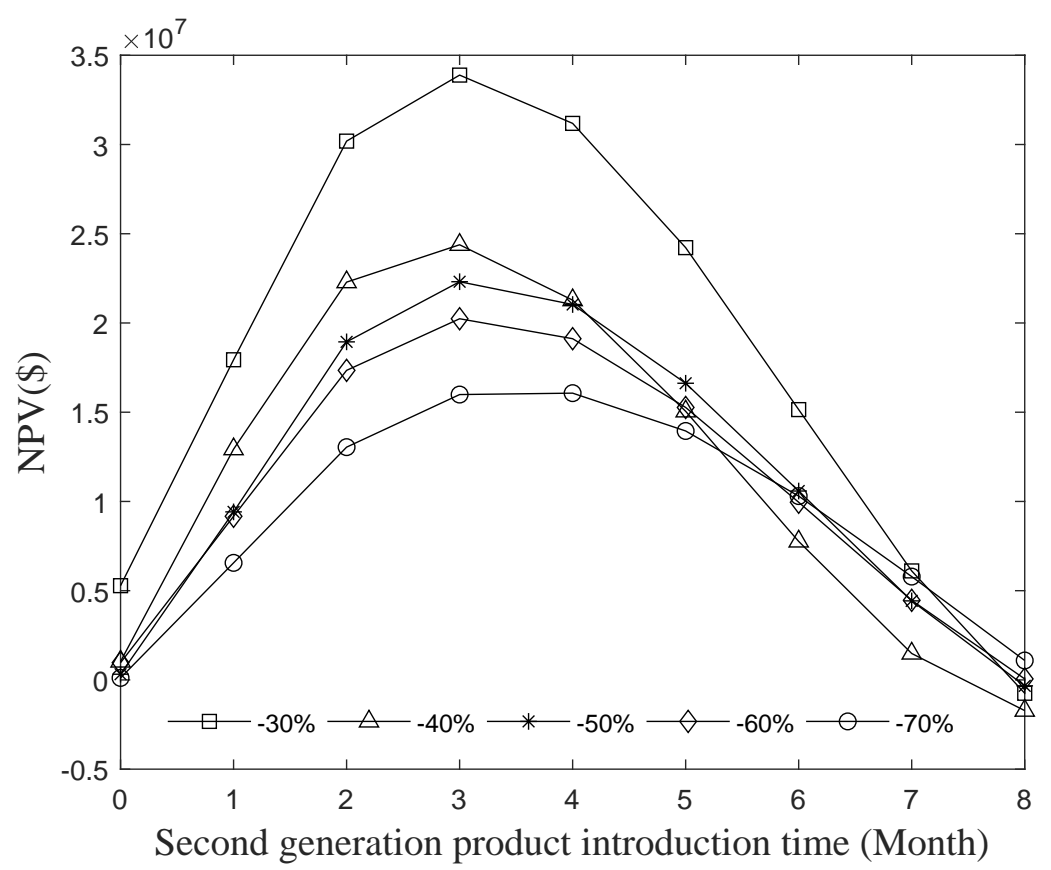

Figure 5.8: Second-generation PIT with Approach (II) (Decreasing pricing policy-phase out transition)

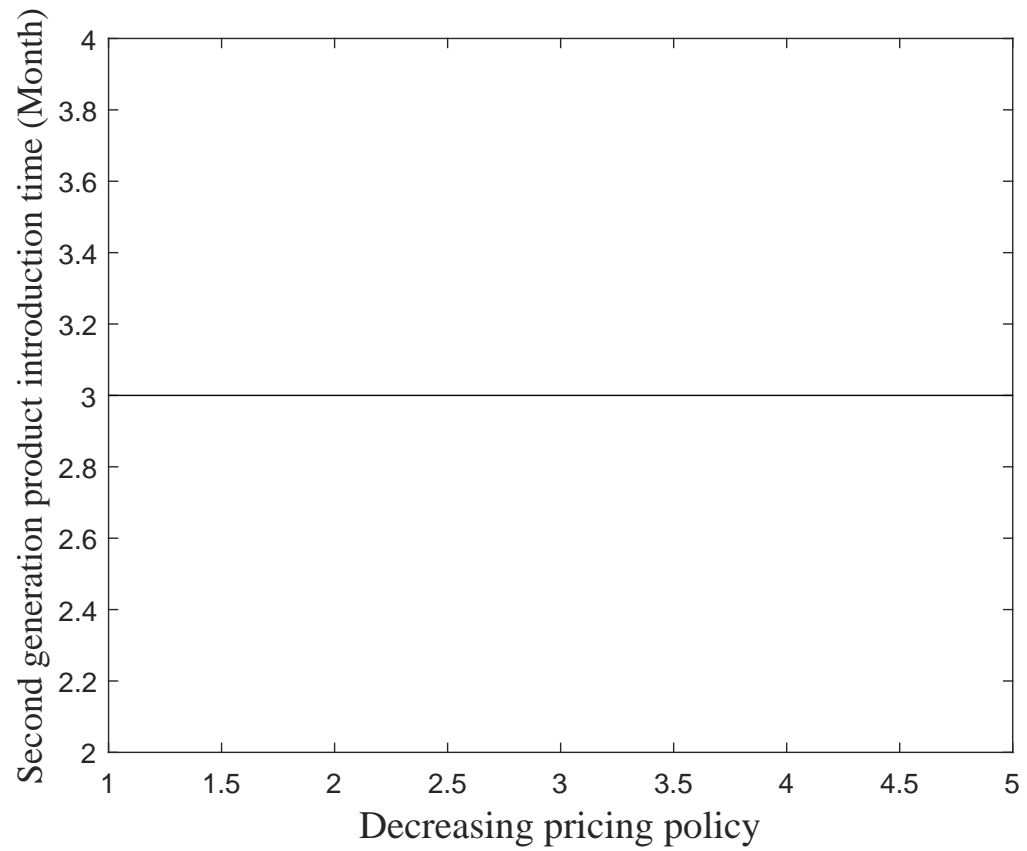

Figure 5.9: Second-generation PIT with Approach (II) (Decreasing pricing policy-phase out transition) 


\subsubsection{Phase out transition in increasing-decreasing pricing policy when decreasing pricing rate is changed}

In Figure 5.10, $t=0$ shows the result of Scenario 3, phase out transition-simultaneous strategy, and $t=1$ to $t=17$ show the results of Scenario 2, phase out transition-sequential strategy. Figure 5.10, shows the increasing-decreasing pricing policy when the price changes only the drift rate. Figure 5.10 shows the results when the increasing rate is 0.05 and the decreasing rate changes from -0.3 to -0.7 . In this scenario, the NPV reaches a peak value of $\$ 400$ million. Figure 5.11 shows that when the decreasing pricing rate increases from -0.7 to -0.3 , the second-generation PIT is delayed from month 7 to month 10 . This is so that the first-generation product lasts longer in the market.

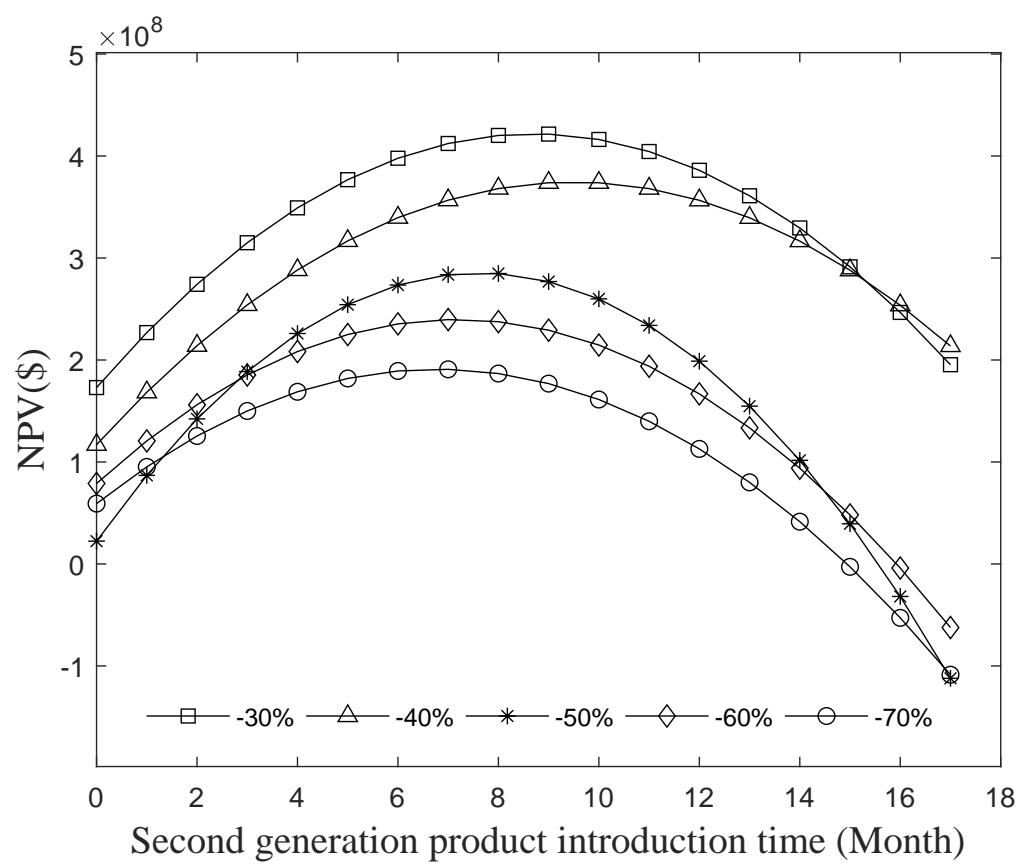

Figure 5.10: Second-generation PIT with Approach (I) (Increasing-Decreasing pricing policy-phase out transition) 


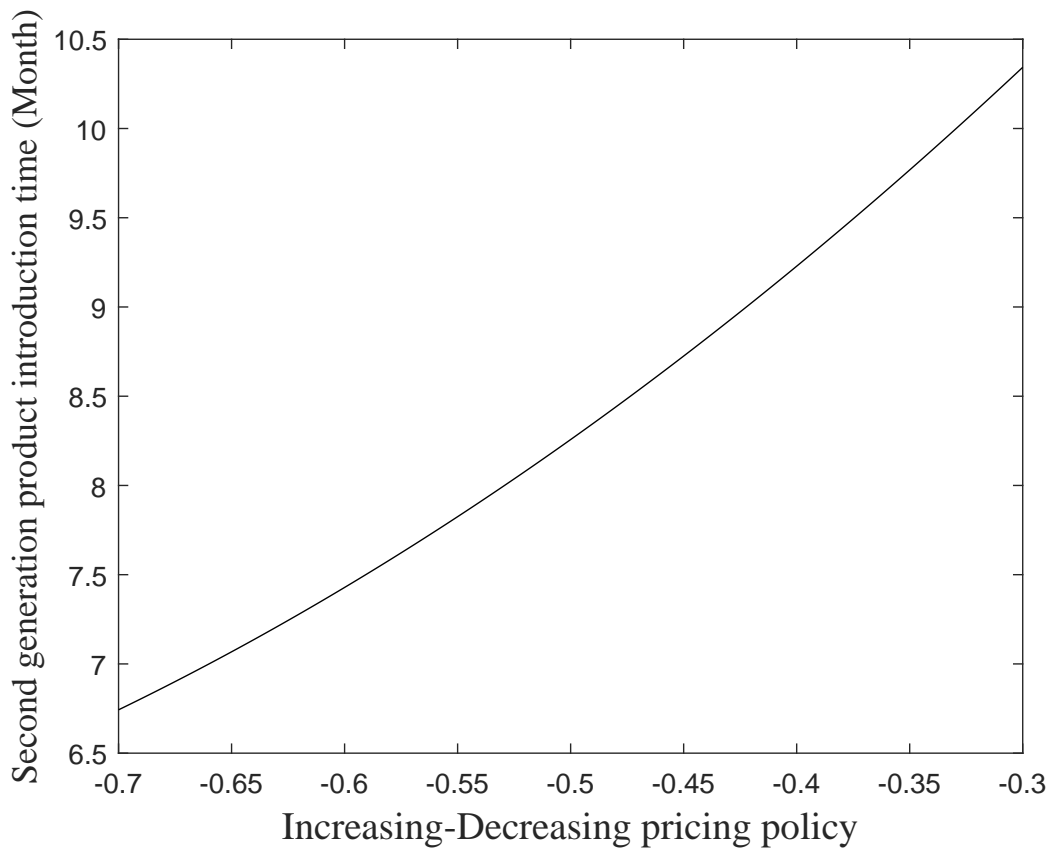

Figure 5.11: Second-generation PIT with Approach (I) (Increasing-Decreasing pricing policy-phase out transition)

In Figure 5.12, $t=0$ shows the result of Scenario 3, phase out transition-simultaneous strategy, and $t=1$ to $t=9$ show the results of Scenario 2, phase out transition-sequential strategy. Figure 5.12 shows the increasing-decreasing pricing policy when the price changes both the drift rate and volatility. Figure 5.12 shows the results when the increasing rate is 0.05 and the decreasing rate changes from -0.3 to -0.7 . In this scenario, the NPV reaches a peak value of $\$ 350$ million. Figure 5.13 shows when the decreasing pricing rate increases from -0.7 to -0.3 , demand for product $B$ decreases and the second-generation PIT is delayed from month 4 to month 5 . In this scenario, first-generation product lasts longer in the market because the decreasing rate of the second-generation product price increases. 


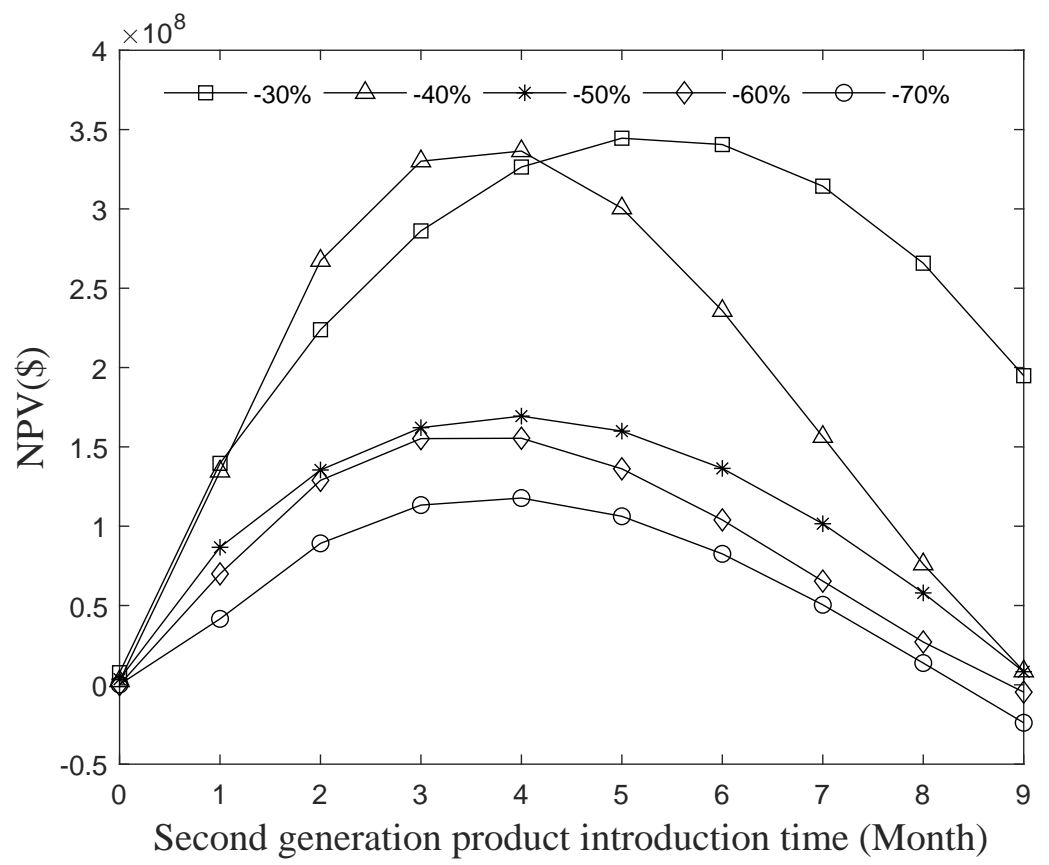

Figure 5.12: Second-generation PIT with Approach (II) (Increasing-Decreasing pricing policy-phase out transition)

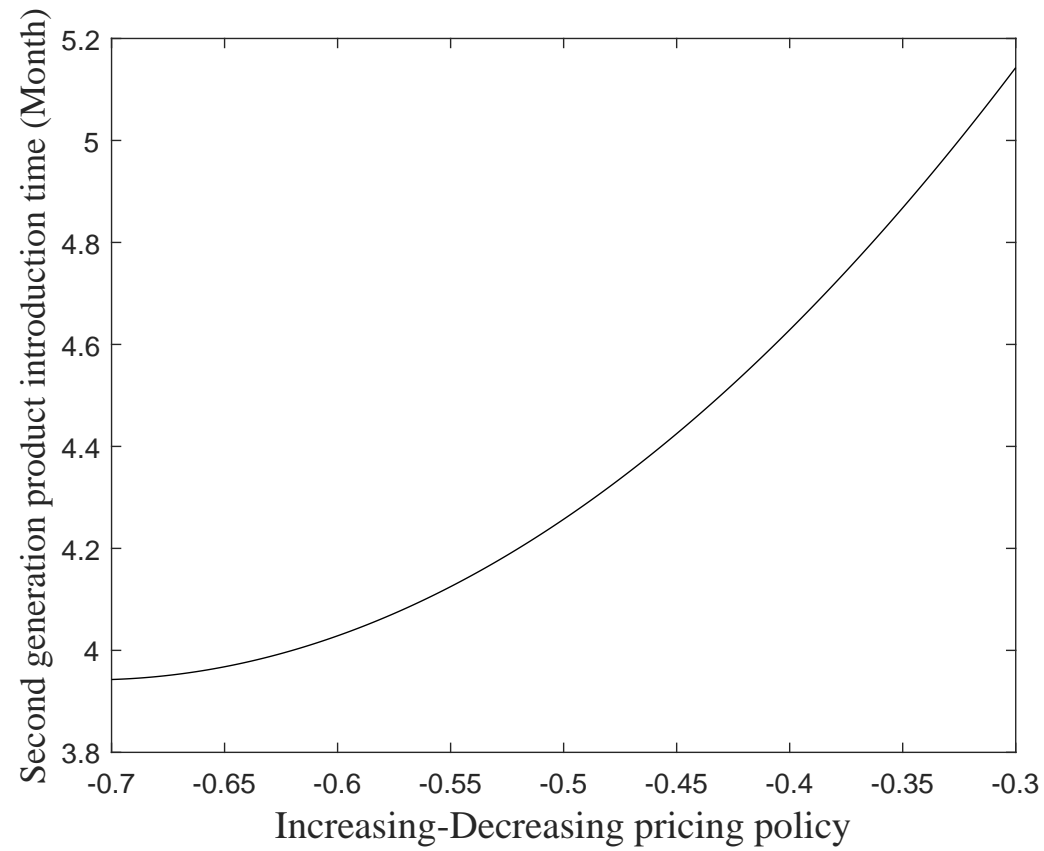

Figure 5.13: Second-generation PIT with Approach (II) (Increasing-Decreasing pricing policy-phase out transition) 


\subsubsection{Phase out transition in increasing-decreasing pricing policy when increasing pricing rate is changed}

The following result shows the increasing-decreasing pricing policy when the increasing rate changes from 0.01 to 0.09 and decreasing rate is -0.5 . In Figure 5.14, $t=0$ shows the result of Scenario 3, phase out transition-simultaneous strategy, and $t=1$ to $t=9$ show the results of Scenario 2, phase out transition-sequential strategy. Figure 5.14 shows the increasing-decreasing pricing policy when the price changes only the drift rate. Figure 5.14 shows the results when the decreasing rate is -0.5 and the increasing rate changes from 0.01 to 0.09. In this scenario, the NPV reaches a peak value of $\$ 790$ million. Figure 5.15 shows when the increasing pricing rate increases from 0.01 to 0.09 , demand for product $B$ decreases and then the second-generation PIT is delayed from month 2 to month 5. In this scenario, the first-generation product lasts longer in the market because the increasing rate of the second-generation product price is increasing.

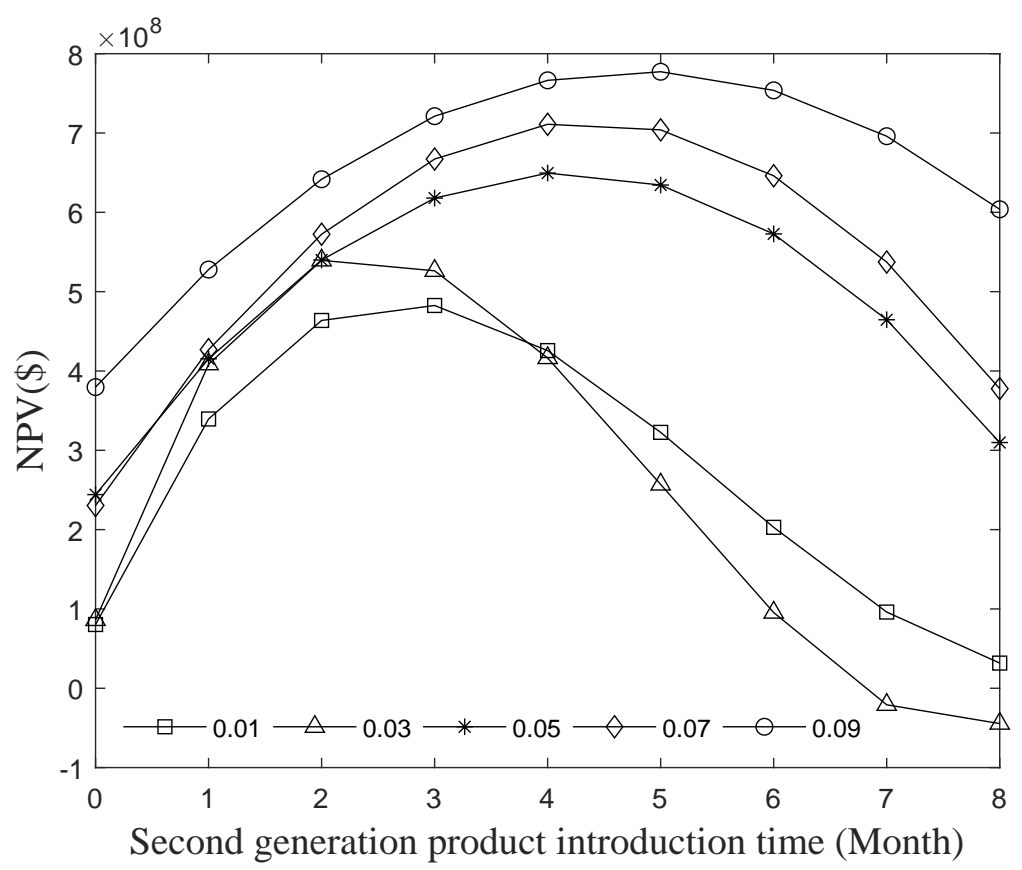

Figure 5.14: Second-generation PIT with Approach (I) (Increasing-Decreasing pricing policy-phase out transition) 


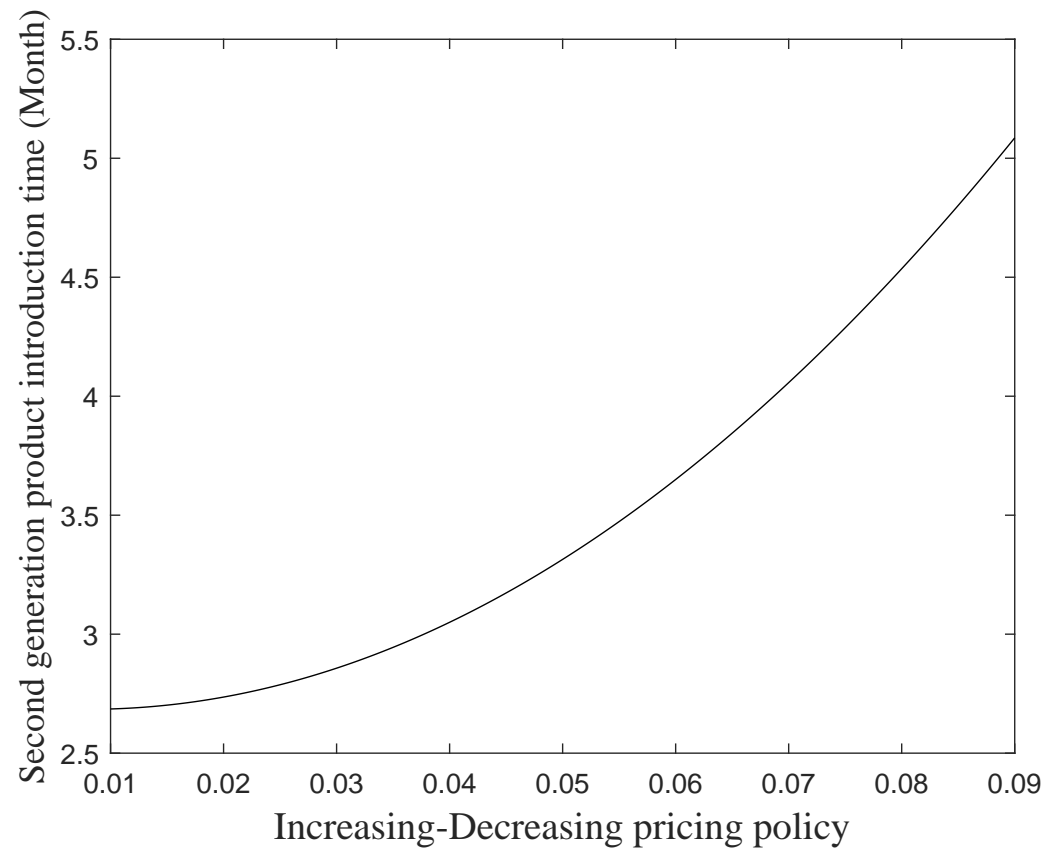

Figure 5.15: Second-generation PIT with Approach (I) (Increasing-Decreasing pricing policy-phase out transition)

In Figure 5.16, $t=0$ shows the result of Scenario 3, phase out transition-simultaneous strategy, and $t=1$ to $t=9$ show the results of Scenario 2, phase out transition-sequential strategy. Figure 5.16 shows the increasing-decreasing pricing policy when the price changes both the drift rate and volatility. Figure 5.16 shows the results when the decreasing rate is -0.5 and the increasing rate changes from 0.01 to 0.09 . In this scenario, the NPV reaches a peak value of $\$ 800$ million. Figure 5.17 shows when the increasing pricing rate increases from 0.01 to 0.09 , the second-generation PIT is delayed from month 4 to month 6 due to decreasing demand for product $B$. In this scenario, as the increasing rate of the secondgeneration product price increases, the first-generation product lasts longer in the market. 


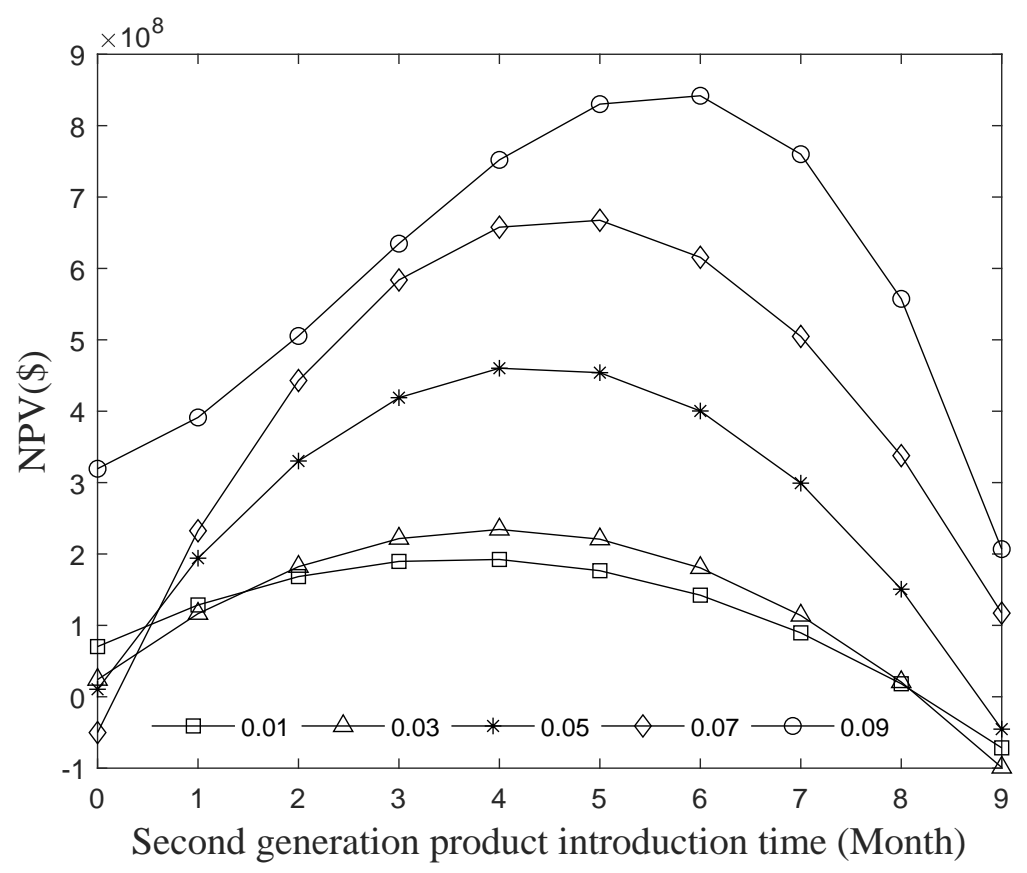

Figure 5.16: Second-generation PIT with Approach (II) (Increasing-Decreasing pricing policy-phase out transition)

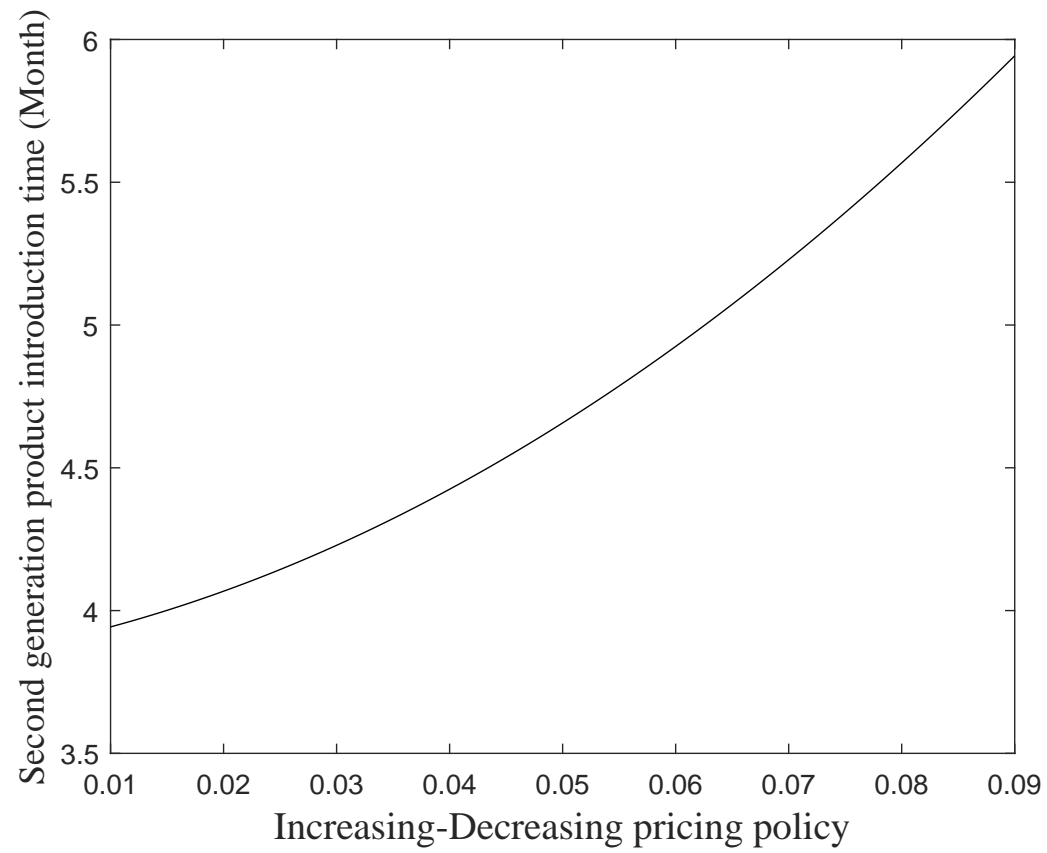

Figure 5.17: Second-generation PIT with Approach (II) (Increasing-Decreasing pricing policy-phase out transition) 


\subsection{Summary}

This chapter has investigated the incorporation of timing and pricing into the process of finding the best second-generation PIT. The motivation is to maximize the NPV over a given period of time. The developed model in this chapter utilizes the GBM to determine the demand parameters including the drift rate, volatility, and correlation of each product. Dynamic programming is used in a lattice approach to maximize the NPV. This chapter examined two different pricing policies. Decreasing and increasing-decreasing pricing policies are two different pricing policies that were considered. These two different pricing policies are investigated in the three different scenarios described in Section 3.2. Moreover, these three different scenarios are analyzed in two different approaches. In the first approach, the price changes only the drift rate, and in the second approach the price changes both drift rate and volatility. Results demonstrate that in decreasing pricing policy, the second-generation product has to be introduced as early as the first-generation product. In increasing-decreasing pricing policy, as the decreasing pricing rate increases from $-70 \%$ to $-30 \%$, the second-generation PIT is delayed. Furthermore, in increasing-decreasing pricing policy, as the increasing pricing rate increases from 0.01 to 0.09 , the second-generation PIT is delayed. Sensitivity analysis is done with respect to different approaches. In the next chapter, the effects of PLC, pricing, and advertising on second-generation PIT are investigated. 


\section{Chapter 6}

\section{The Valuation of Product}

\section{Introduction Strategies Considering}

\section{PLC, Pricing and Advertising}

\subsection{Introduction}

Advertising is one of the marketing mix variables that affect demand diffusion; furthermore, different marketing strategies affect demand. Of the two main types of advertising, generic advertising affects the sales market, while brand advertising affects market share (Niu, 2006). Bass et al. (2005) studied how much budget should be allocated to brand advertising and generic advertising. Generic advertising targets consumer beliefs about the product category, while the brand advertising provides consumers with information about the brand's value. Generic advertising (static case) affects increasing sales and the brand advertising (dynamic case) affects the market share.

In economics, a monopoly is defined as a single producer of a product or service. Even a monopoly firm like Apple company needs to advertise. By advertising, companies can increase the sales and also they can increase awareness about their product and persuade the potential buyers to buy a product (Bass et al., 2005). A monopoly firm uses advertising to 
differentiate its products and build the brand identity. By advertising a product, a monopoly firm can increase the perceptions of the product distinction.

Budgeting of advertising has been studied in papers such as Fairhurst et al. (1996), and Miller and Pazgal (2007). The percentages of sales and objectives are two commonly used approaches for determining advertising expenditures. This research uses the percentage of sales method. A specific percentage of dollar sales is considered under the percentage of sales method, while in the objective method, firms allocate advertising expenses to follow a specific sales level (Miller and Pazgal, 2007). Percentage of sales is one of the most widely used approaches for determining advertising expenditures (Miller and Pazgal, 2007). The percentage of advertising budget varies for each industry. For many industries, 10\% of a product's sales is used for advertising expenses. As the PLC changes over time, advertising strategies are also changing. Furthermore, the demand for each product affects the other one, which complicates the advertising strategies. Thus, finding the best product introduction time is a crucial task for many companies, especially considering the marketing mix variables. An overview of the third phase of the research is shown in Figure 6.1

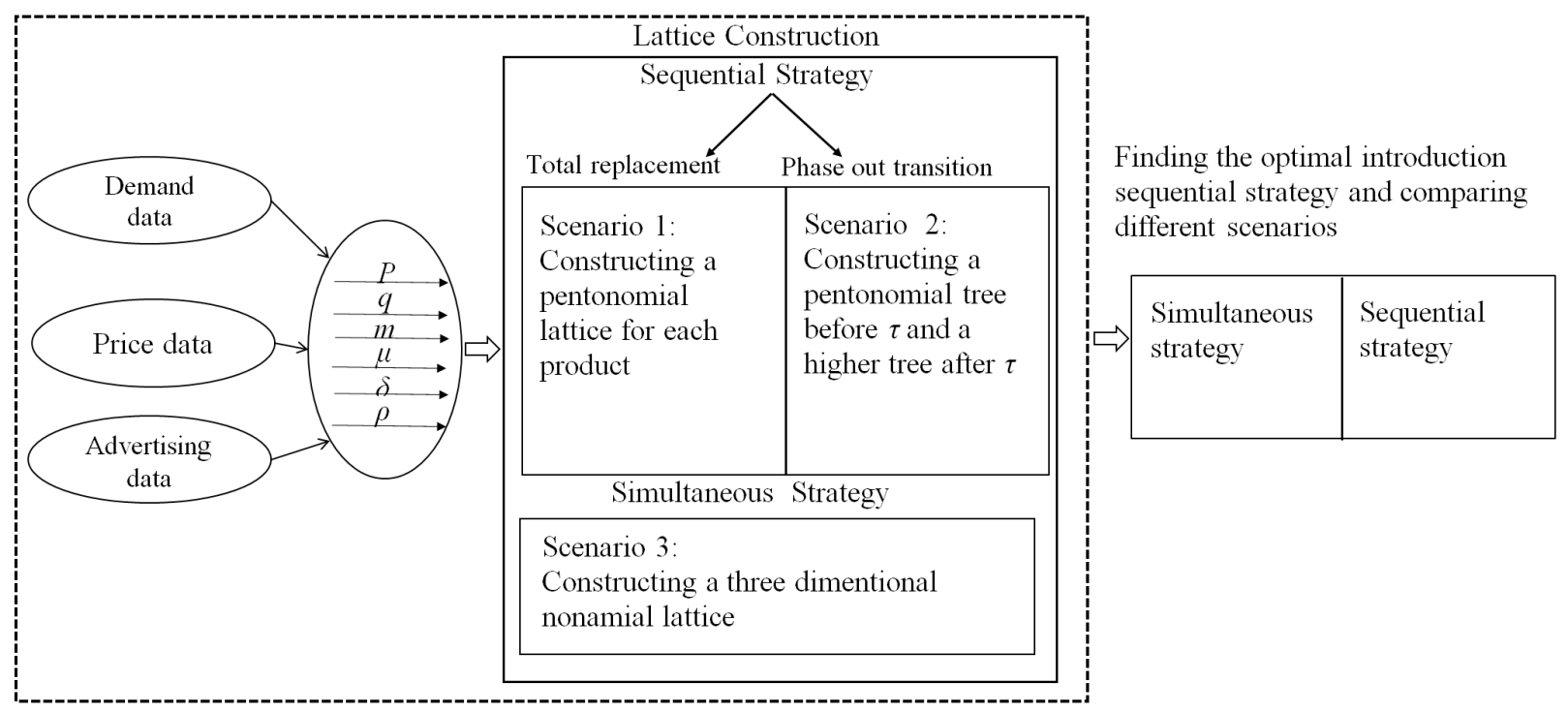

Figure 6.1: Overall framework of methodology 
The main focus of this research is to find the best second-generation product introduction time for generic advertising in high-tech and durable products. By using the developed methodology in this research, a company can determine how much money they could make when demand is uncertain for the specific percentage of advertisement budgeting.

\subsection{Model development}

In this section, the steps to propose the third model are described. In the first step, Bass parameters (innovation coefficient, $p$, imitation coefficient, $q$, and market size, $m$ ) are estimated using the method explained in Section 3.2.1 and Equation (6.3). The stochastic demand parameters (i.e., drift rate, volatility, and correlation) are then calculated using the diffusion adoption function of each product and calculating the demand probability distribution function (Jiang and Jain, 2012a). Details of estimating the stochastic demand parameters are described in Section 6.3. Finally, the lattice approach is used to model product demands; detailed steps are described in Section 4.4. Furthermore, a dynamic programming approach is used to find the maximum NPV over a given period.

\subsection{Estimating the stochastic demand parameters}

The demand probability distribution function for each product is calculated by using the following diffusing adoption function (Jiang and Jain, 2012a) and Bass parameters from the following equations.

$$
F_{i}(t)= \begin{cases}0 & \text { if } t<0 \\ \frac{1-e^{-\left(p_{i}+q_{i}\right) X_{i}(t)}}{\left(q_{i} / p_{i}\right) e^{-\left(p_{i}+q_{i}\right) X_{i}(t)}+1} & \text { if } t \geq 0\end{cases}
$$

then

$$
f_{i}(t)=\frac{\left(p_{i}+q_{i}\right)^{2}}{p_{i}} x_{i}(t) \frac{e^{-\left(p_{i}+q_{i}\right) X_{i}(t)}}{\left[\left(q_{i} / p_{i}\right) e^{-\left(p_{i}+q_{i}\right) X_{i}(t)}+1\right]^{2}},
$$


and sales at $t$

$$
S_{i}(t)=m_{i} \frac{\left(p_{i}+q_{i}\right)^{2}}{p_{i}} x_{i}(t) \frac{e^{-\left(p_{i}+q_{i}\right) X_{i}(t)}}{\left[\left(q_{i} / p_{i}\right) e^{-\left(p_{i}+q_{i}\right) X_{i}(t)}+1\right]^{2}},
$$

where $p_{i}$ and $q_{i}$ are the coefficient of innovation and imitation, respectively for product $\mathrm{i}$ $(i \in\{A, B\})$ and $X_{i}(t)$ is the price function (cumulative marketing effect), where $X_{i}(t)$

$$
\begin{gathered}
X_{i}(t)=t+\beta_{1_{i}} \ln \left[\frac{P r_{i}(t)}{\operatorname{Pr}_{i}(0)}\right]+\beta_{2_{i}} \ln \left[\frac{A d_{i}(t)}{A d_{i}(0)}\right], \\
x_{i}(t)=1+\beta_{1_{i}}\left[\frac{\operatorname{Pr}_{i}^{\prime}(t)}{P r_{i}(t)}\right]+\beta_{2_{i}}\left[\frac{A d_{i}^{\prime}(t)}{A d_{i}(t)}\right] .
\end{gathered}
$$

Where $x_{i}(t)$ is the current marketing effect and $\operatorname{Pr}_{i}^{\prime}(t)$ and $A d_{i}^{\prime}(t)$ are the rate of change in the price and advertising, respectively. $\operatorname{Pr}_{i}(t)$ and $\operatorname{Pr}_{i}(0)$ are the price of each product at time $t$ and zero, respectively. Furthermore, $A d_{i}(t)$ and $A d_{i}(0)$ are the advertising of each product at time $t$ and zero, respectively. $\beta_{1_{i}}$ and $\beta_{2_{i}}$ are the diffusion price and diffusion advertising parameters respectively and represent the effectiveness of price and advertising over time.

The sales price and demand data for Dram $4 \mathrm{~K}$ and $16 \mathrm{~K}$ are used for analysis (Norton and Bass, 1987). Equation (6.3) is used to estimate the Bass parameters for products $A$ and B. Table 6.1 shows the estimated Bass parameters for Products $A$ and $B$.

Table 6.1: Estimated Bass parameters for products $A$ and $B$

\begin{tabular}{|l||l|l|}
\hline Bass Parameters & Product $A$ & Product $B$ \\
\hline$p$ & 0.0123 & 0.0547 \\
$q$ & 0.936 & 0.957 \\
$m$ & 320 & 1306 \\
$\beta_{1}$ & -4.55 & -3.23 \\
$\beta_{2}$ & 4.53 & 3.62 \\
\hline
\end{tabular}

The following steps show the calculation of the stochastic demand parameters. There are two products $A$ and $B$ and each product could be in the growth or the decaying regime. 
Each product regime is defined by a GBM as below:

$$
\theta^{A}(t)=\text { Demand of product } A=\left\{\begin{array}{cc}
\theta_{g}^{A} & \text { if } t \leq t_{A} \\
\theta_{d}^{A} & \text { if } t>t_{A}
\end{array}\right.
$$

similarly, for product $B$

$$
\theta^{B}(t)=\text { Demand of product } B=\left\{\begin{array}{ccc}
\theta_{g}^{B} & \text { if } t \leq t_{B} \\
\theta_{d}^{B} & \text { if } t>t_{B}
\end{array}\right.
$$

The switching times for products $A$ and $B$ from growth to decay regime are denoted by $t_{A}$ and $t_{B}$, respectively. When the price affects only drift rate, we have:

$$
\begin{aligned}
& \frac{\mathrm{d} \theta_{g}^{A}(t)}{\theta_{g}^{A}(t)}=\left(\mu_{g}^{A}+X^{\prime}(t)\right) \mathrm{d} t+\sigma_{g}^{A} \mathrm{~d} W_{t}^{A, g}, \mu_{g}^{A}>0, \\
& \frac{\mathrm{d} \theta_{d}^{A}(t)}{\theta_{d}^{A}(t)}=\left(\mu_{d}^{A}+X^{\prime}(t)\right) \mathrm{d} t+\sigma_{d}^{A} \mathrm{~d} W_{t}^{A, d}, \mu_{d}^{A}<0, \\
& \frac{\mathrm{d} \theta_{g}^{B}(t)}{\theta_{g}^{B}(t)}=\left(\mu_{g}^{B}+X^{\prime}(t)\right) \mathrm{d} t+\sigma_{g}^{B} \mathrm{~d} W_{t}^{B, g}, \mu_{g}^{B}>0, \\
& \frac{\mathrm{d} \theta_{d}^{B}(t)}{\theta_{d}^{B}(t)}=\left(\mu_{d}^{B}+X^{\prime}(t)\right) \mathrm{d} t+\sigma_{d}^{A} \mathrm{~d} W_{t}^{B, d}, \mu_{d}^{B}<0 .
\end{aligned}
$$

When the price affects both drift rate and volatility, we have (see Appendix C for details)

$$
\begin{aligned}
& \frac{\mathrm{d} \theta_{g}^{A}(t)}{\theta_{g}^{A}(t)}=\left[\mu_{g}^{A} X(t)+X^{\prime}(t)+\frac{X^{2}(t)}{2} \sigma_{g}^{2 A}\right] d t+X(t) \sigma_{g}^{A} \mathrm{~d} W_{t}^{A, g}, \mu_{g}^{A}>0, \\
& \frac{\mathrm{d} \theta_{d}^{A}(t)}{\theta_{d}^{A}(t)}=\left[\mu_{d}^{A} X(t)+X^{\prime}(t)+\frac{X^{2}(t)}{2} \sigma_{d}^{2 A}\right] d t+X(t) \sigma_{d}^{A} \mathrm{~d} W_{t}^{A, d}, \mu_{d}^{A}<0, \\
& \frac{\mathrm{d} \theta_{g}^{B}(t)}{\theta_{g}^{B}(t)}=\left[\mu_{g}^{B} X(t)+X^{\prime}(t)+\frac{X^{2}(t)}{2} \sigma_{g}^{2 B}\right] d t+X(t) \sigma_{g}^{B} \mathrm{~d} W_{t}^{B, g}, \mu_{g}^{B}>0, \\
& \frac{\mathrm{d} \theta_{d}^{B}(t)}{\theta_{d}^{B}(t)}=\left[\mu_{d}^{B} X(t)+X^{\prime}(t)+\frac{X^{2}(t)}{2} \sigma_{d}^{2 B}\right] d t+X(t) \sigma_{d}^{B} \mathrm{~d} W_{t}^{B, d}, \mu_{d}^{B}<0 .
\end{aligned}
$$


For identifying the characteristics of the product life cycle, historical data is used for each product; in this way, the product regimes are identified. The following steps show how the demand parameters are estimated. As each product is represented by a GBM, the growth regime of the product $\mathrm{A}$ is defined as

$$
U_{\mathrm{g}}^{A}(t)=\operatorname{Ln}\left[\frac{\theta_{\mathrm{g}}^{A}(t)}{\theta_{\mathrm{g}}^{A}(t-1)}\right],
$$

and average of $U_{\mathrm{g}}^{A}(t)$ is

$$
\bar{U}_{\mathrm{g}}^{A}(t)=(1 / n) \sum_{t=1}^{n} U_{\mathrm{g}}^{A}(t) .
$$

Then, drift rate, $\mu_{g}^{A}$, can be estimated as

$$
\bar{U}_{g}^{A}(t) / h
$$

Similarly, we can define $\mu_{d}^{A}, \mu_{g}^{B}$, and $\mu_{d}^{B}$. $h$ is the time in year between two consecutive observations. Next, $\sigma_{\mathrm{g}}^{A}$, the volatility of product $\mathrm{A}$ in the growth regime, can be found in Ross (1999) and Hull (2009) as

$$
\sigma_{\mathrm{g}}^{A} / \sqrt{h}
$$

where

$$
\sigma_{\mathrm{g}}^{A}=\sqrt{(1 / n-1) \sum_{\mathrm{t}=1}^{n}\left(U_{\mathrm{g}}^{A}(t)-\bar{U}_{\mathrm{g}}^{A}(t)\right)^{2}} .
$$

Estimation of other regime parameters can be calculated as above. Similarly, we can define $\sigma_{d}^{A}, \sigma_{g}^{B}$, and $\sigma_{d}^{B}$. The correlation between demands in a combined regime $\left(A_{g}, B_{g}\right)$ can be calculated as

$$
\rho_{g g}=\left[\sum_{\mathrm{t}=1}^{n}\left(U_{\mathrm{g}}^{A}(t)-\bar{U}_{\mathrm{g}}^{A}(t)\right) \cdot\left(U_{\mathrm{g}}^{B}(t)-\bar{U}_{\mathrm{g}}^{B}(t)\right)\right] /(n-1) \sigma_{\mathrm{g}}^{A} \sigma_{\mathrm{g}}^{B}
$$

similarly, for other combined regimes $\left(A_{d}, B_{g}\right),\left(A_{g}, B_{d}\right)$, and $\left(A_{d}, B_{d}\right)$ we have $\rho_{d g}, \rho_{g d}$, 
and $\rho_{d d}$, respectively.

$$
\begin{aligned}
& \rho_{d g}=\left[\sum_{\mathrm{t}=1}^{n}\left(U_{\mathrm{d}}^{A}(t)-\bar{U}_{\mathrm{d}}^{A}(t)\right) \cdot\left(U_{\mathrm{g}}^{B}(t)-\bar{U}_{\mathrm{g}}^{B}(t)\right)\right] /(n-1) \sigma_{\mathrm{d}}^{A} \sigma_{\mathrm{g}}^{B}, \\
& \rho_{g d}=\left[\sum_{\mathrm{t}=1}^{n}\left(U_{\mathrm{g}}^{A}(t)-\bar{U}_{\mathrm{g}}^{A}(t)\right) \cdot\left(U_{\mathrm{d}}^{B}(t)-\bar{U}_{\mathrm{d}}^{B}(t)\right)\right] /(n-1) \sigma_{\mathrm{g}}^{A} \sigma_{\mathrm{d}}^{B}, \\
& \rho_{d d}=\left[\sum_{\mathrm{t}=1}^{n}\left(U_{\mathrm{d}}^{A}(t)-\bar{U}_{\mathrm{d}}^{A}(t)\right) \cdot\left(U_{\mathrm{d}}^{B}(t)-\bar{U}_{\mathrm{d}}^{B}(t)\right)\right] /(n-1) \sigma_{\mathrm{d}}^{A} \sigma_{\mathrm{d}}^{B} .
\end{aligned}
$$

Demand parameters for products $A$ and $B$ are estimated using estimated Bass parameters from Equation (6.3) and the method is explained above. Sales for the first- and secondgeneration products are calculated by using Equations (6.1 and 6.2) and following equations.

$$
S_{A}(t)= \begin{cases}m_{A} f_{A}(t) & \text { if } t<\tau \\ m_{A} f_{A}(t)\left[1-F_{B}(t-\tau)\right], & \text { if } t \geq \tau\end{cases}
$$

and for the second-generation product we have

$$
S_{B}(t)=\left[m_{B}+m_{A} F_{A}(t)\right] f_{B}(t-\tau)+m_{A} f_{A}(t) F_{B}(t-\tau) \text {, if } t \geq \tau \text {. }
$$

Table 6.2 shows the estimated drift rate and volatility for product $A$.

Table 6.2: Estimated demand parameters for product $A$

\begin{tabular}{|l||l|l|}
\hline Parameters & Growth regime & Decay regime \\
\hline Drift rate & 0.071885 & -0.017823 \\
Volatility & 0.005503 & 0.027263 \\
\hline
\end{tabular}

Table 6.3 shows the estimated drift rate and volatility for product $B$. 
Table 6.3: Estimated demand parameters for product $B$

\begin{tabular}{|l||l|l|}
\hline Parameters & Growth regime & Decay regime \\
\hline Drift rate & 0.053654 & -0.029402 \\
Volatility & 0.005043 & 0.037437 \\
\hline
\end{tabular}

Table 6.4 shows the estimated correlation of combined regimes $\left(A_{g}, B_{g}\right),\left(A_{d}, B_{g}\right),\left(A_{g}, B_{d}\right)$, and $\left(A_{d}, B_{d}\right)$.

Table 6.4: Estimated correlated demand for combined regimes

\begin{tabular}{|l|l|l|l|}
\hline$\rho_{g g}$ & $\rho_{g d}$ & $\rho_{d g}$ & $\rho_{d d}$ \\
\hline 0.331251 & -0.715348 & -0.489944 & 0.606219 \\
\hline
\end{tabular}

\subsection{Analysis and results}

We examined the three scenarios described in the methodology section with two different pricing policies considered in this research: decreasing and increasing-decreasing pricing policies. For the first product, always decreasing pricing policy is considered, while for the second product either decreasing or increasing-decreasing pricing policy is considered. In increasing-decreasing pricing policy, the change rate of the increasing part remains the same in the increasing and decreasing parts. A decreasing pricing policy attracts more price sensitive consumers Bolton (1989). In contrast, an increasing-decreasing pricing policy draws consumers who are not as price sensitive as those of the decreasing pricing policy Shankar and Krishnamurthi (1996).

These three scenarios and two different pricing policies are examined in two different approaches. In the first approach, Approach (I), the price changes only the drift rate, and in the second approach, Approach (II), the price changes both drift rate and volatility. The sales price and demand data for Dram $4 \mathrm{~K}$ and $16 \mathrm{~K}$ are used in analysis (Norton and Bass, 
1987). Based on the literature, the most commonly used method for advertising budget is percentage of sales price, which is around $10 \%$ for many industries. In all the following scenarios, the advertising budget is considered to be $10 \%$ of price sales.

\subsubsection{Total replacement}

In Scenario 1, total replacement, decreasing pricing policy and $10 \%$ advertising budget, Figure 6.2 shows that as the pricing decreasing rate decreases from -0.3 to -0.7 the secondgeneration product introduction time will be delayed. As the pricing decreasing rate decreases, the second-generation product lasts longer in the market. Figure 6.3 shows that as the second-generation product price decreases from -0.3 to -0.7 , the second-generation product introduction time increases from month 4 to month 6 . Accordingly, product $B$ is expected to last longer in the market. This highlights that product $B$ remains in the market for 2 more months.

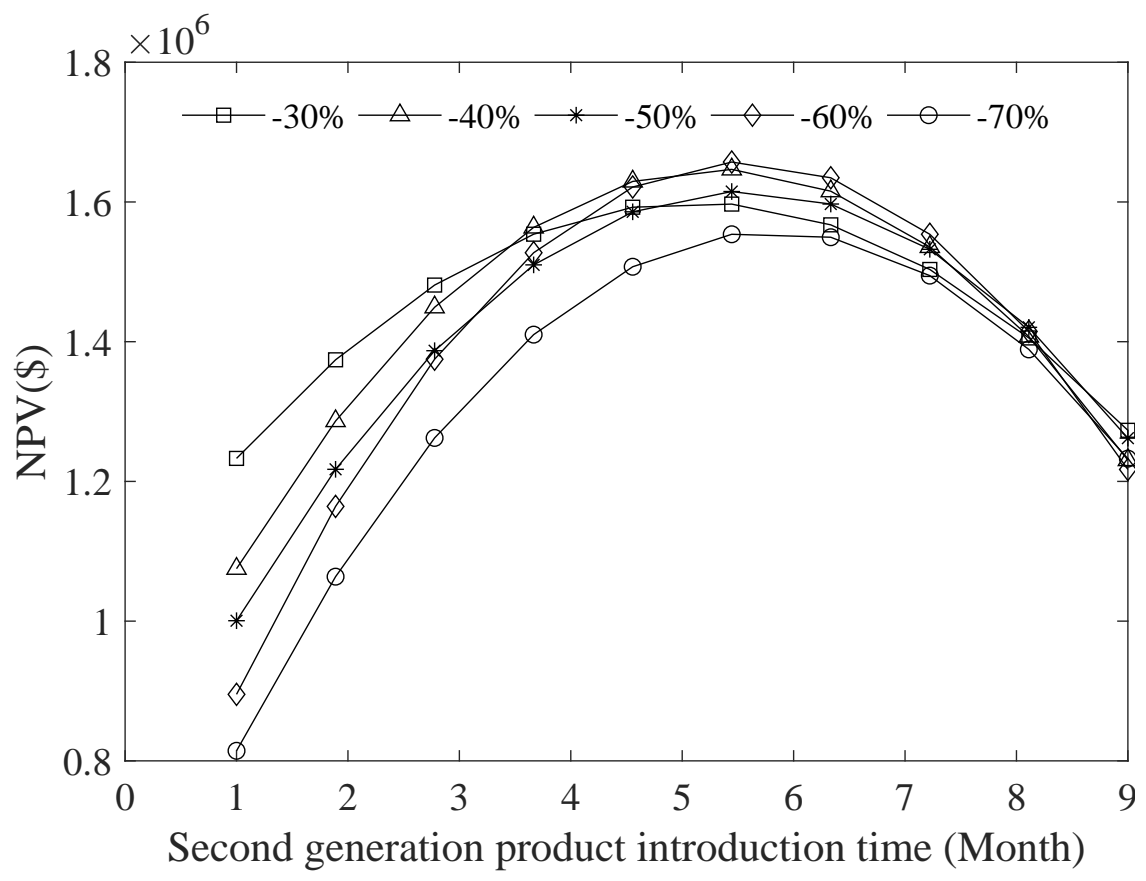

Figure 6.2: Second-generation product introduction time (Decreasing pricing policy-total replacement) 


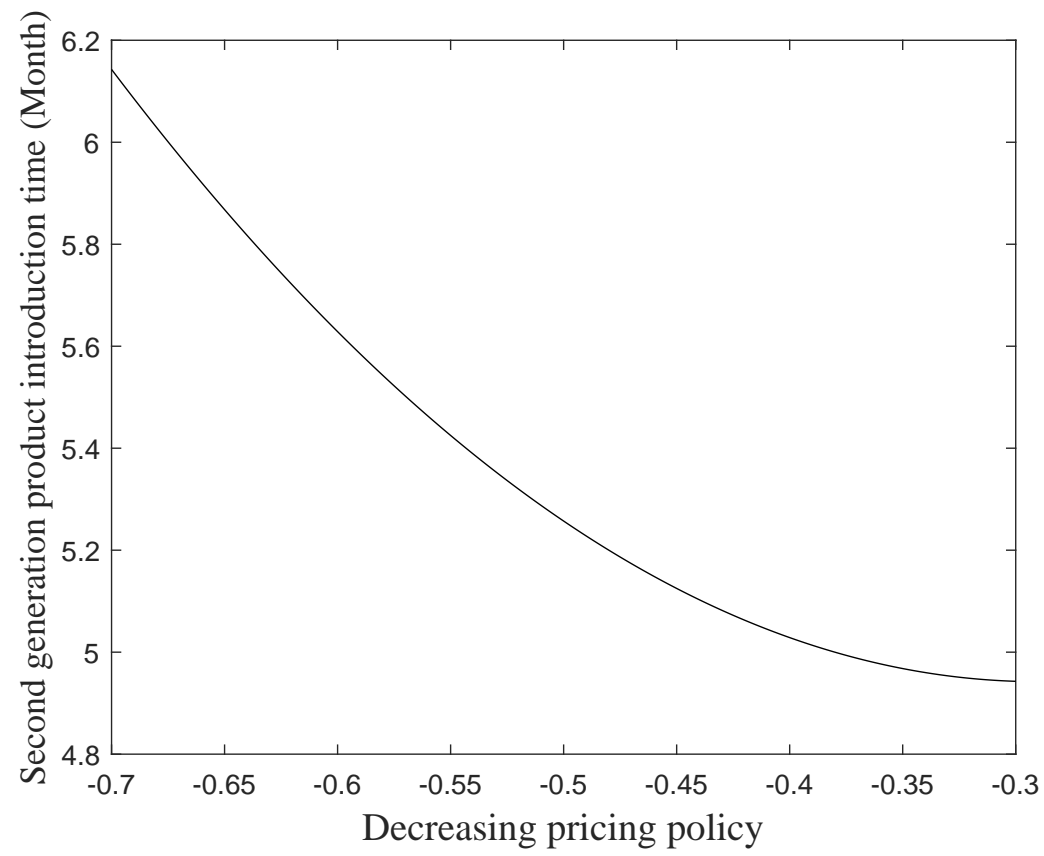

Figure 6.3: Second-generation product introduction time (Decreasing pricing policy-total replacement)

In Scenario 1, total replacement, increasing-decreasing pricing policy and $10 \%$ advertising budget, Figure 6.4 shows the results when the increasing rate is 0.05 and the decreasing rate changes from -0.3 to -0.7 . When the decreasing pricing rate decreases from -0.3 to -0.7 , the second-generation product introduction time is delayed. As the pricing increasing-decreasing rate decreases, the second-generation product lasts longer in the market. Figure 6.5 shows the results when increasing-decreasing pricing policy decreases from -0.3 to -0.7 . As the second-generation product price decreases from -0.3 to -0.7 , the second-generation product introduction time increases from month 4 to month 6 . This highlights that product $B$ remains in the market for 2 more months since product $B$ is expected to last longer in the market. 


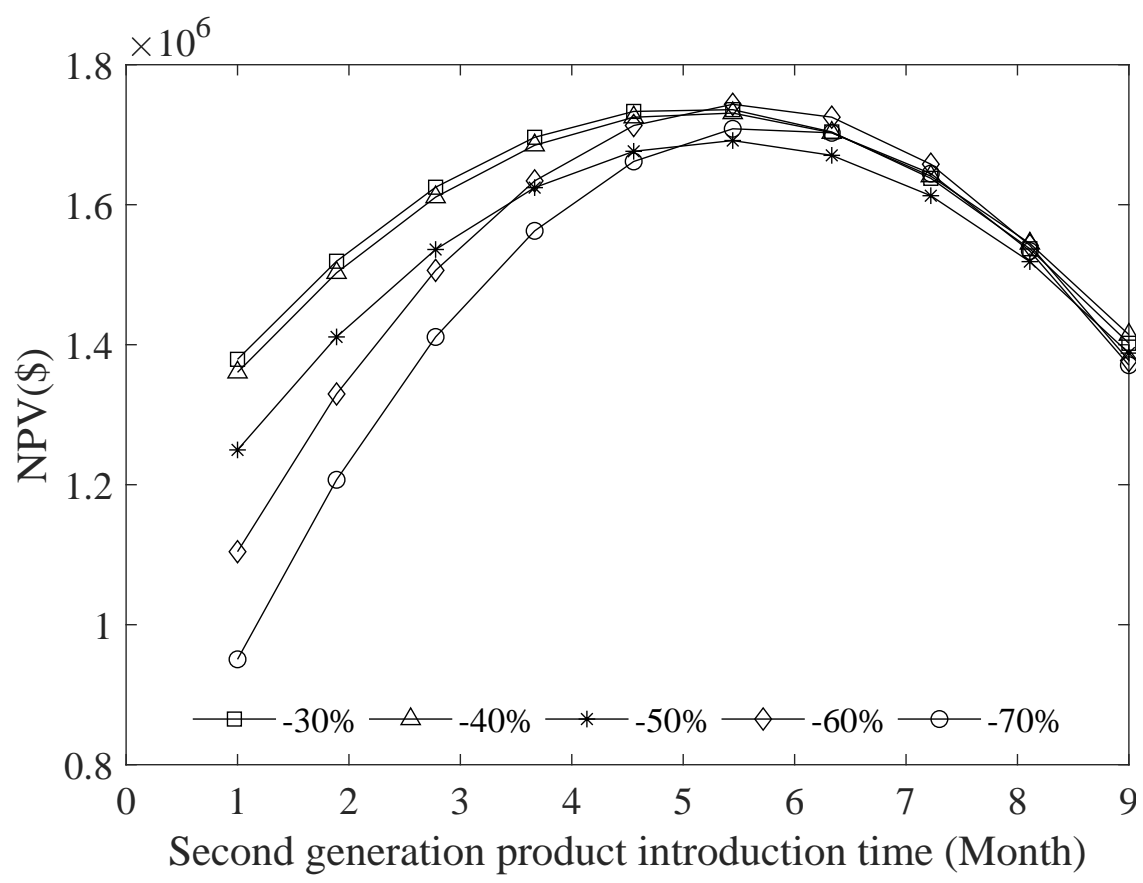

Figure 6.4: Second-generation product introduction time (Increasing-Decreasing pricing policy-total replacement)

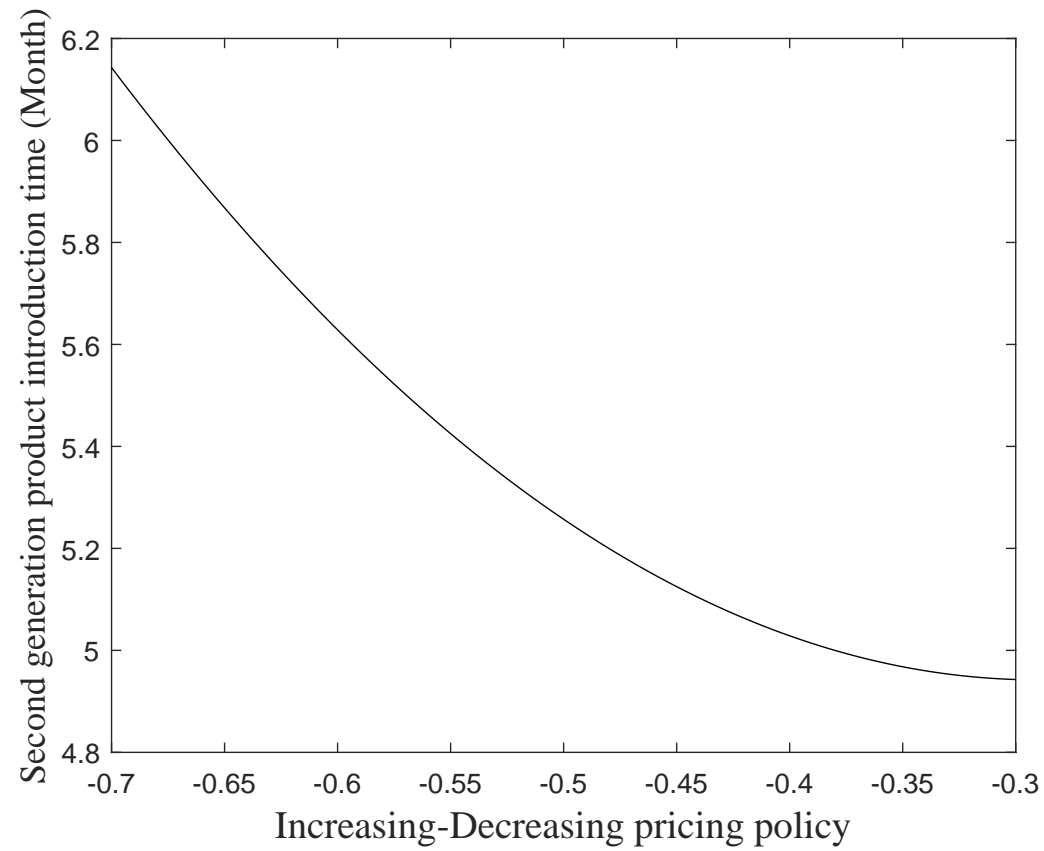

Figure 6.5: Second-generation product introduction time (Increasing-Decreasing pricing policy-total replacement) 


\subsubsection{Phase out transition}

In Figure 6.6, $t=0$ shows the result of Scenario 3, phase out transition-simultaneous strategy, and $t=1$ to $t=5$ show the results of Scenario 2, phase out transition-sequential strategy. Figure 6.6 shows the decreasing pricing policy when the price changes only the drift rate. When the pricing decreasing rate decreases from -0.3 to -0.7 , the second-generation product introduction time remains the same at month 2.5. In this scenario, the NPV reaches a peak value of $\$ 3.6$ million, and the second-generation product has to be introduced as early as the first-generation product introduction time. Figure 6.7 shows the second-generation product introduction time remains at month 2. This is so that the second-generation product demand increases as the second-generation product pricing policy rate decreases.

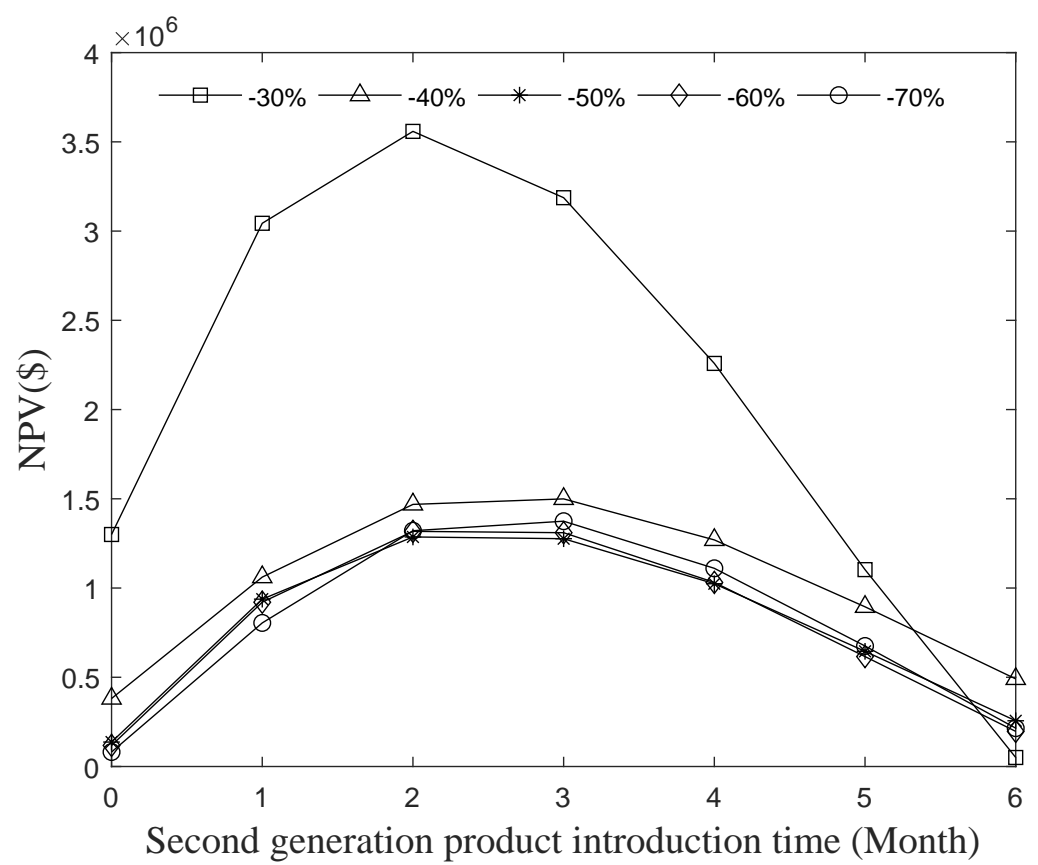

Figure 6.6: Second-generation product introduction time with Approach (I) (Decreasing pricing policy-phase out transition) 


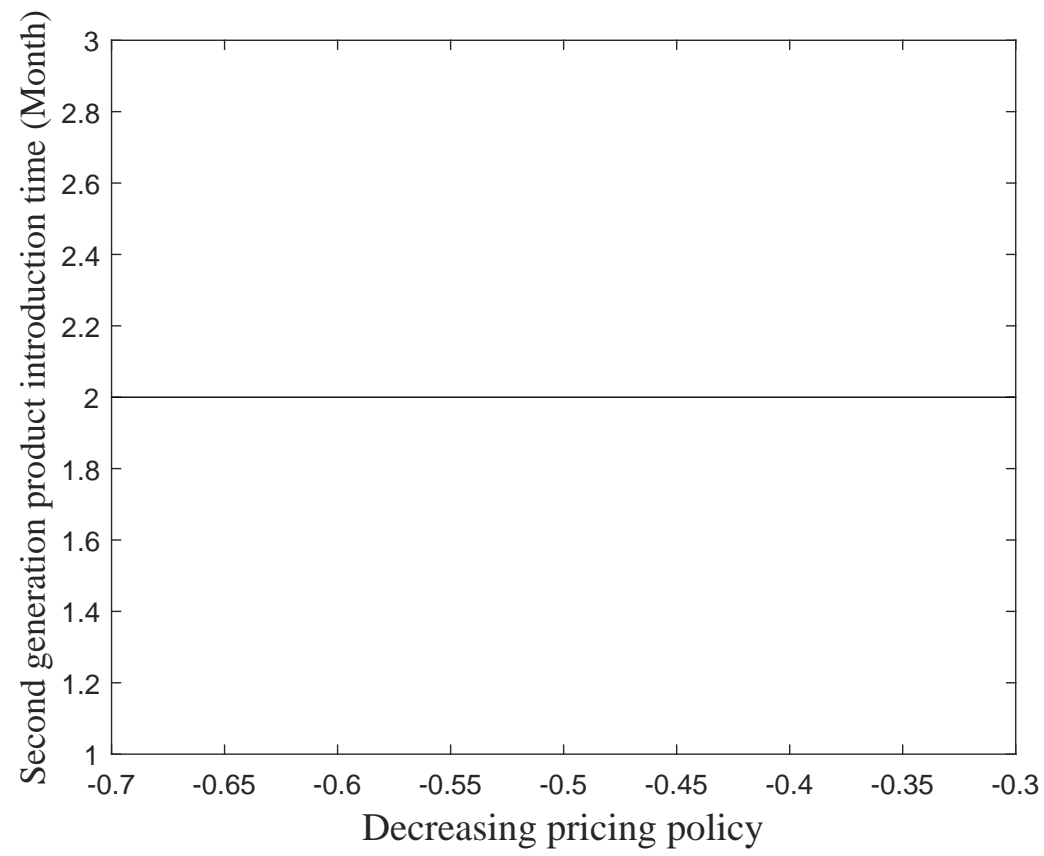

Figure 6.7: Second-generation product introduction time with Approach (I) (Decreasing pricing policy-phase out transition)

In Figure $6.8, t=0$ shows the result of Scenario 3, phase out transition-simultaneous, and $t=1$ to $t=9$ show the results of Scenario 2, phase out transition-sequential strategy. Figure 6.8 shows the decreasing pricing policy when the price changes both drift rate and volatility. When the pricing decreasing rate decreases from -0.3 to -0.7 , the second-generation product introduction time remains the same at month 3. In this scenario, the NPV reaches a peak value of $\$ 12$ million. Figure 6.9 shows the second-generation product introduction time remains at month 3 . It means in this scenario, the second-generation product has to be introduced as early as the first-generation product introduction time. As the secondgeneration product pricing policy decreases, demand for product $B$ increases. 


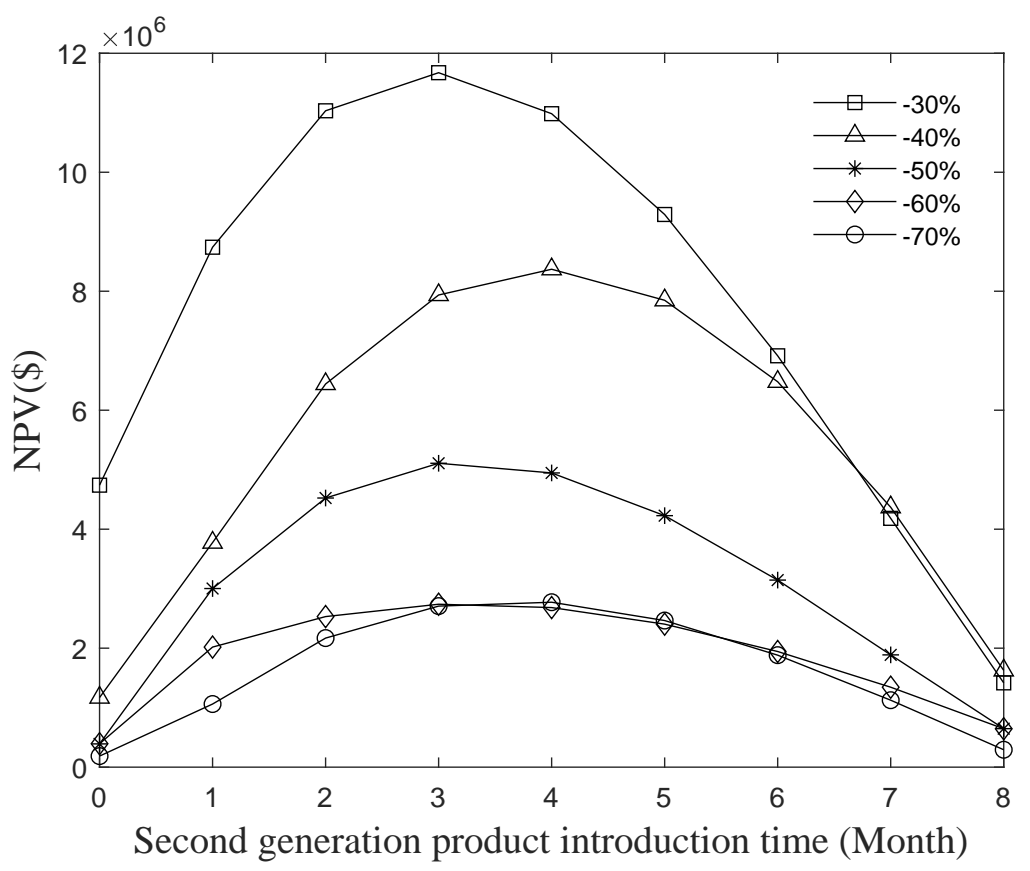

Figure 6.8: Second-generation product introduction time with Approach (II) (Decreasing pricing policy-phase out transition)

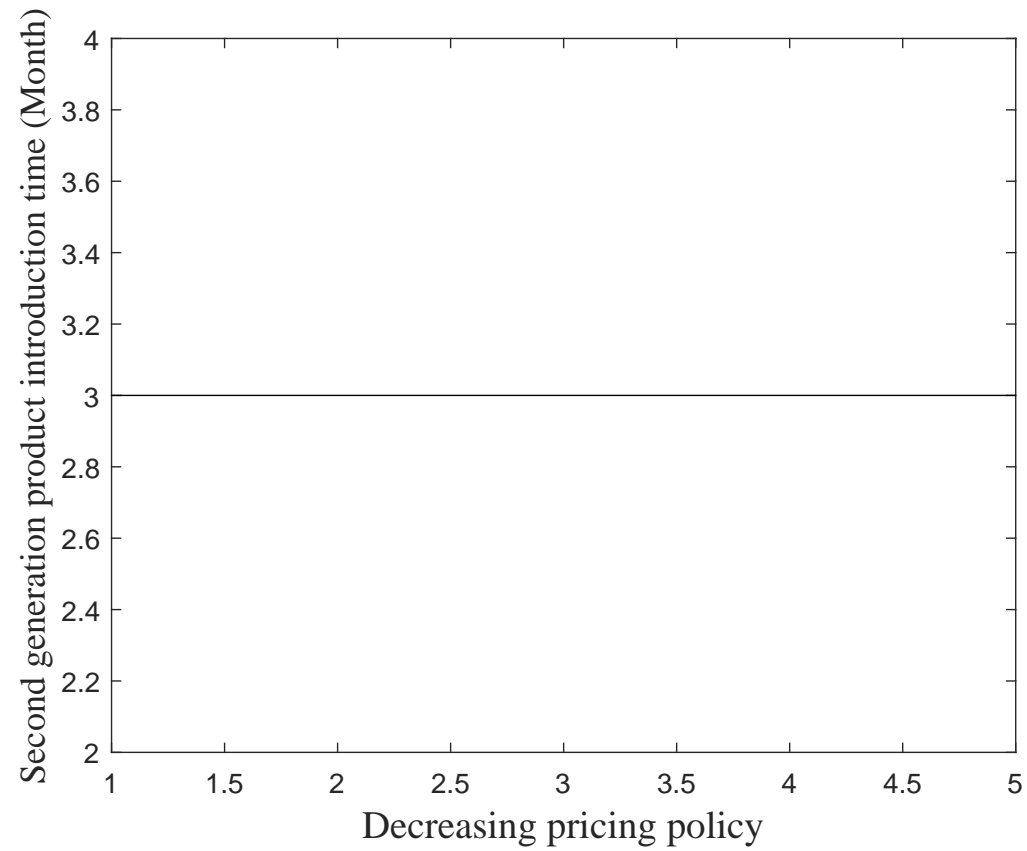

Figure 6.9: Second-generation product introduction time with Approach (II) (Decreasing pricing policy-phase out transition) 


\subsubsection{Phase out transition in increasing-decreasing pricing policy when decreasing pricing rate is changed}

In Figure $6.10, t=0$ shows the result of Scenario 3, phase out transition-simultaneous strategy, and $t=1$ to $t=9$ show the results of Scenario 2, phase out transition-sequential strategy. Figure 6.10 shows the increasing-decreasing pricing policy when the price changes only the drift rate. Figure 6.10 shows the results when the increasing rate is 0.05 and the decreasing rate changes from -0.3 to -0.7 . In this scenario, the NPV reaches a peak value of $\$ 700$ million. Figure 6.11 shows that when the decreasing pricing rate increases from -0.7 to -0.3 , the second-generation product introduction time is delayed from month 4 to month 6 . This is so that the first-generation product lasts longer in the market.

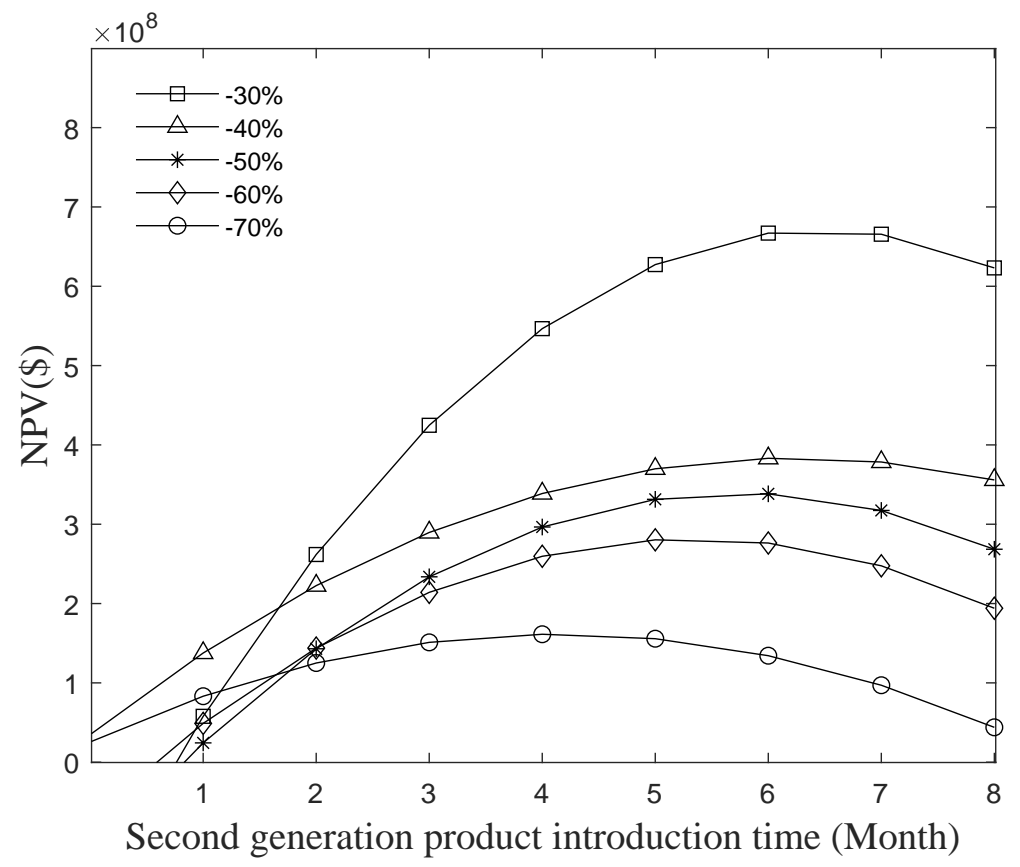

Figure 6.10: Second-generation product introduction time with Approach (I) (IncreasingDecreasing pricing policy-phase out transition) 


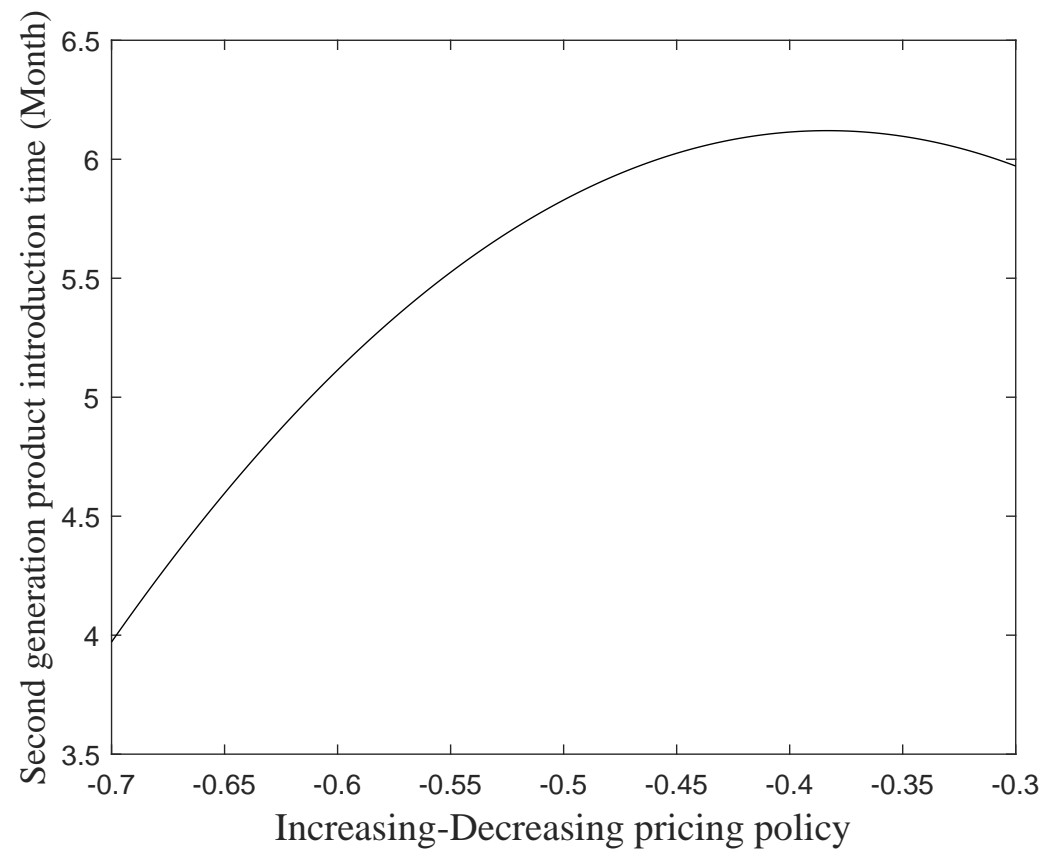

Figure 6.11: Second-generation product introduction time with Approach (I) (IncreasingDecreasing pricing policy-phase out transition)

In Figure 6.12, $t=0$ shows the result of Scenario 3, phase out transition-simultaneous strategy, and $t=1$ to $t=9$ show the results of Scenario 2, phase out transition-sequential strategy. Figure 6.12 shows the increasing-decreasing pricing policy when the price changes both the drift rate and volatility. Figure 6.12 shows the results when the increasing rate is 0.05 and the decreasing rate changes from -0.3 to -0.7 . Figure 6.13 shows when the decreasing pricing rate increases from -0.7 to -0.3 , the second-generation product introduction time is delayed from month 3 to month 8 . In this scenario, first-generation product lasts longer in the market because the decreasing rate of the second-generation product price increases. It means there is more demand for product $A$. 


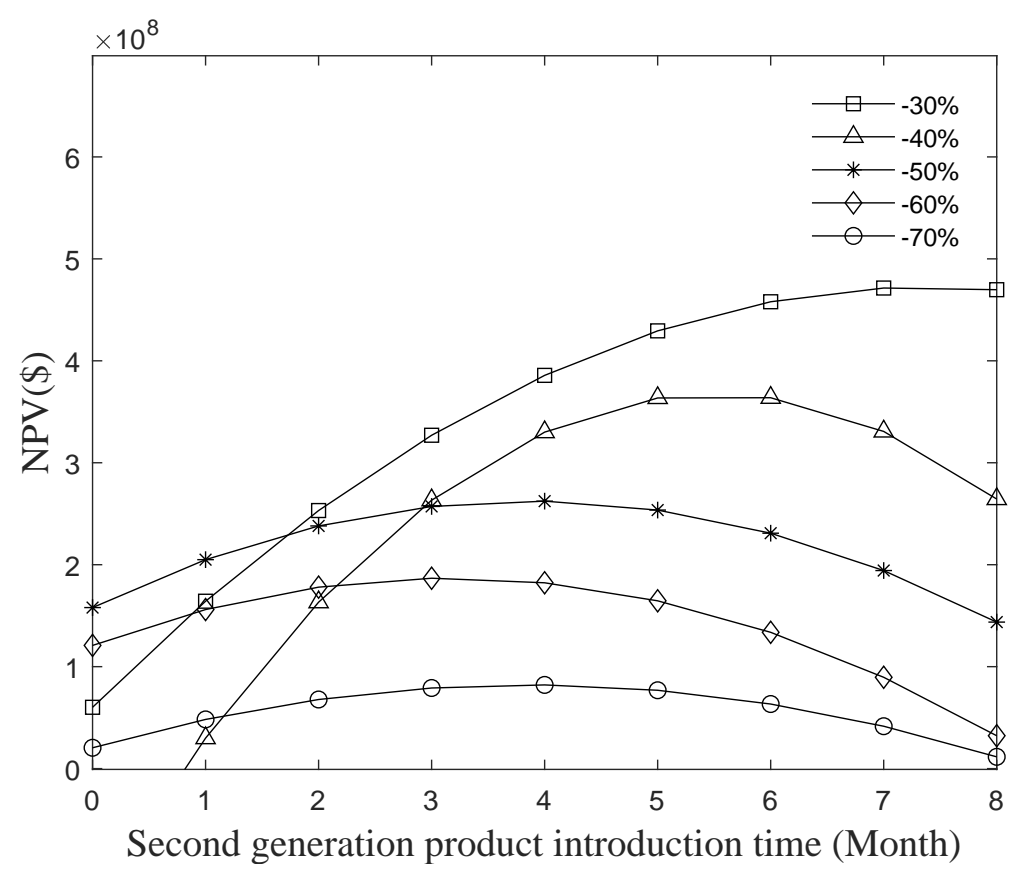

Figure 6.12: Second-generation product introduction time with Approach (II) (IncreasingDecreasing pricing policy-phase out transition)

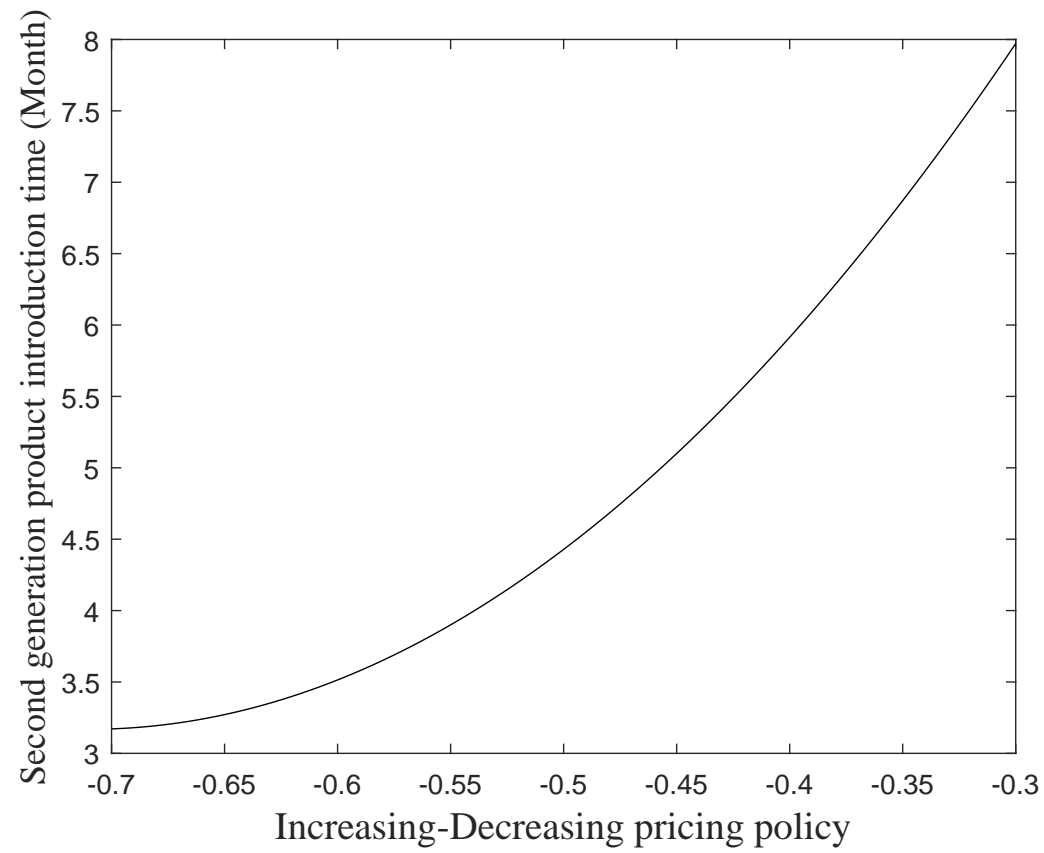

Figure 6.13: Second-generation product introduction time with Approach (II) (IncreasingDecreasing pricing policy-phase out transition) 


\subsubsection{Phase out transition in increasing-decreasing pricing policy when increasing pricing rate is changed}

The following results show the increasing-decreasing pricing policy when the increasing rate is changing from 0.01 to 0.09 and the decreasing rate is -0.5 . In Figure $6.14, t=0$ shows the result of Scenario 3, phase out transition-simultaneous strategy, and $t=1$ to $t=9$ show the results of Scenario 2, phase out transition-sequential strategy. Figure 6.14 shows the increasing-decreasing pricing policy when the price changes only the drift rate. Figure 6.14 shows the results when the decreasing rate is -0.5 and increasing rate changes from 0.01 to 0.09. Figure 6.15 shows when the increasing pricing rate increases from 0.01 to 0.09 , the second-generation product introduction time is delayed from month 3 to month 5 . It means demand for the second-generation product decreases.

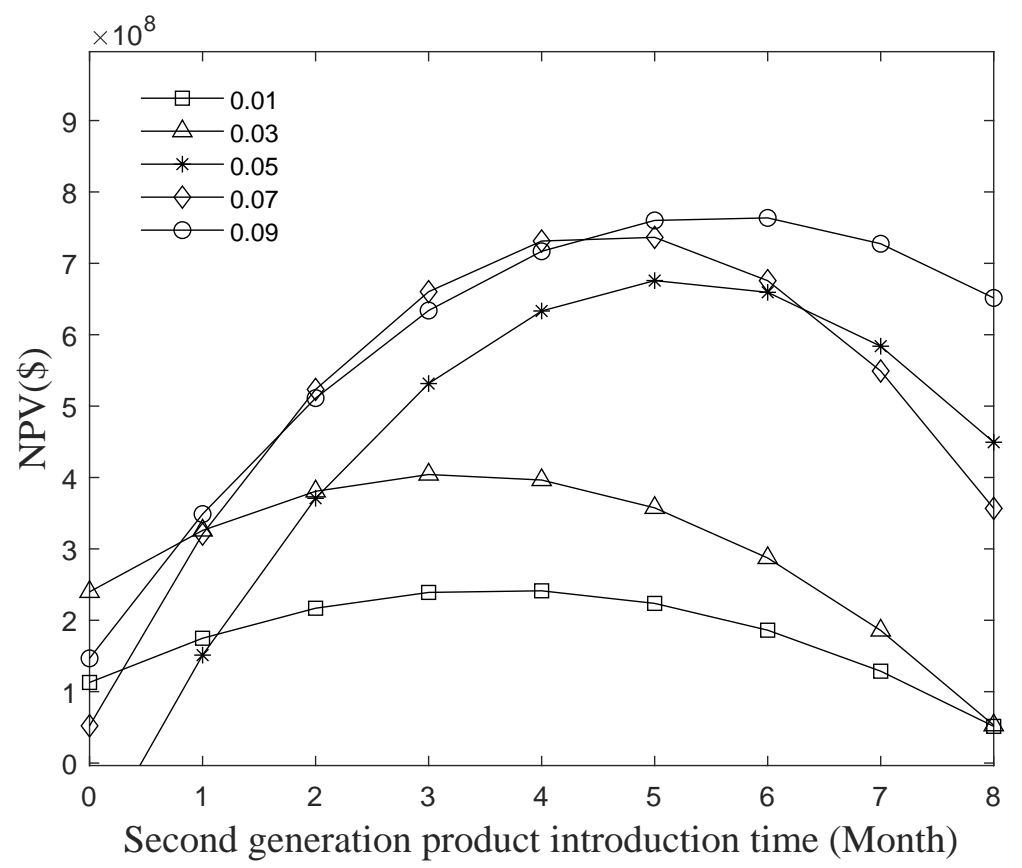

Figure 6.14: Second-generation product introduction time with Approach (I) (IncreasingDecreasing pricing policy-phase out transition) 


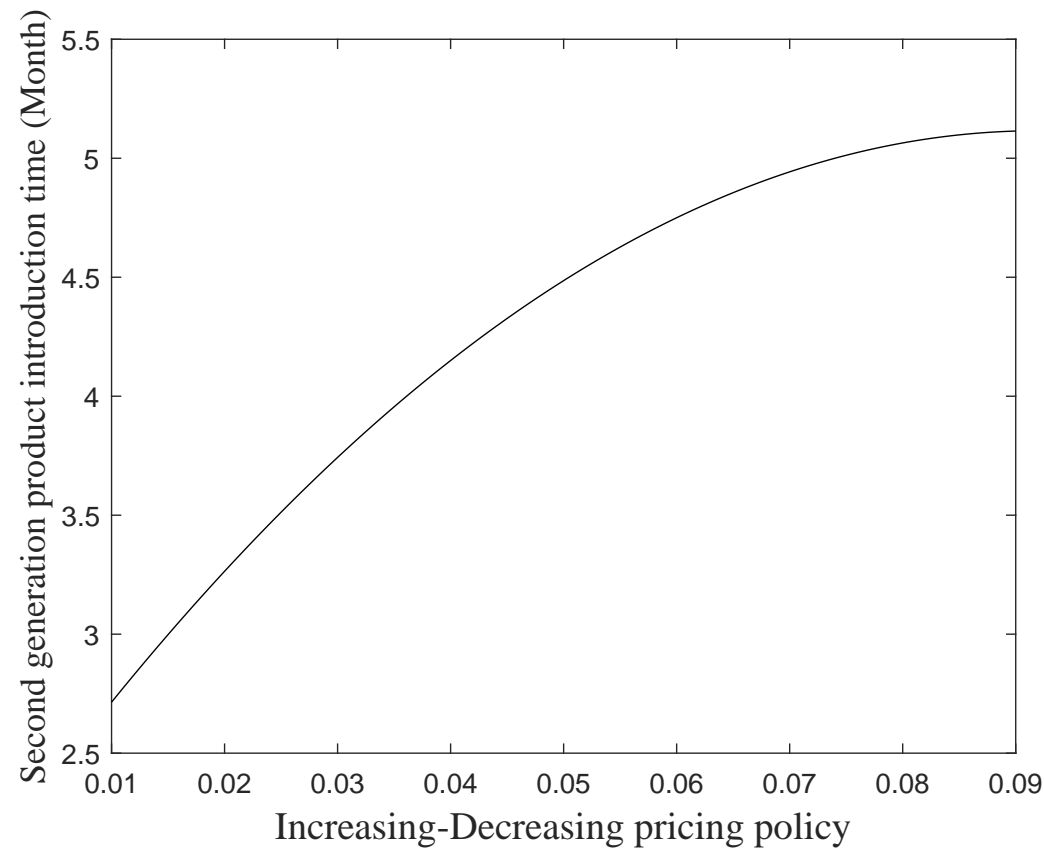

Figure 6.15: Second-generation product introduction time with Approach (I) (IncreasingDecreasing pricing policy-phase out transition)

In Figure 6.16, $t=0$ shows the result of Scenario 3, phase out transition, and $t=1$ to $t=8$ show the results of Scenario 2, phase out transition-sequential strategy. Figure 6.16, shows the increasing-decreasing pricing policy when the price changes both the drift rate and volatility. Figure 6.16 shows the results when the decreasing rate is -0.5 and the increasing rate changes from 0.01 to 0.09 . Figure 6.17 shows when the increasing pricing rate increases from 0.01 to 0.09 , the second-generation product introduction time is delayed from month 3 to month 5. In this scenario, first-generation product lasts longer in the market because the increasing rate of the second-generation product price is increasing. 


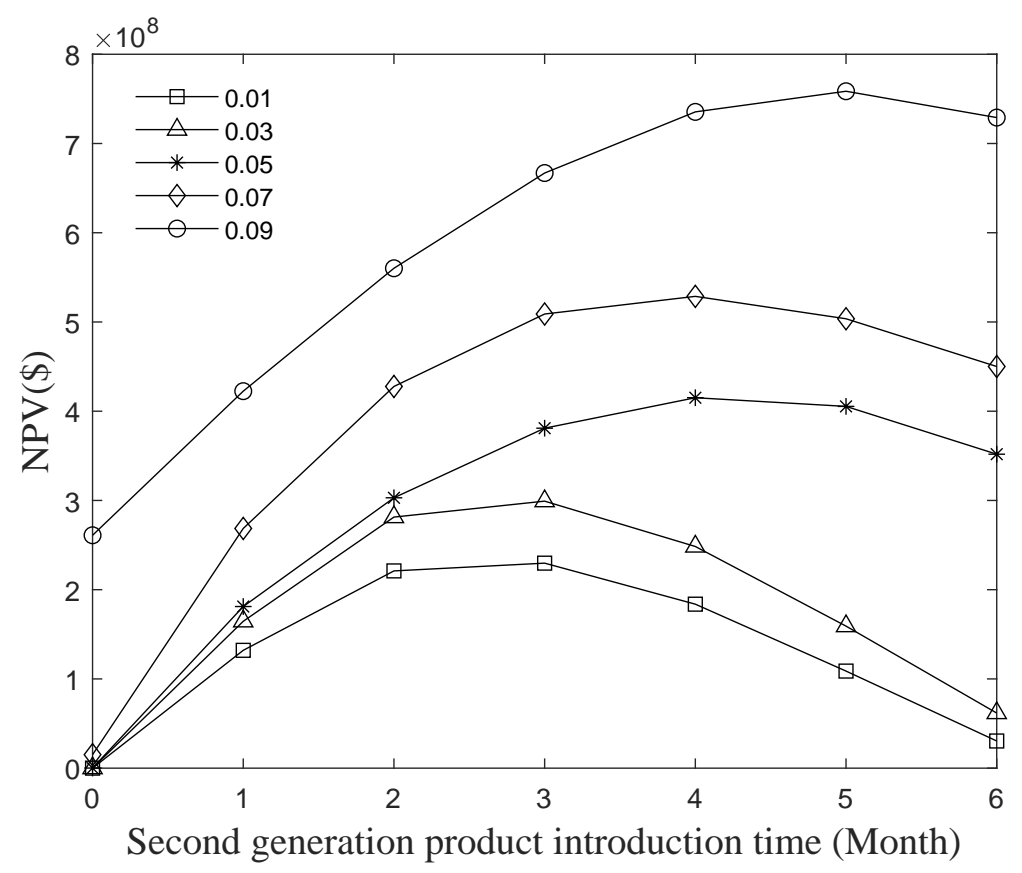

Figure 6.16: Second-generation product introduction time with Approach (II) (IncreasingDecreasing pricing policy-phase out transition)

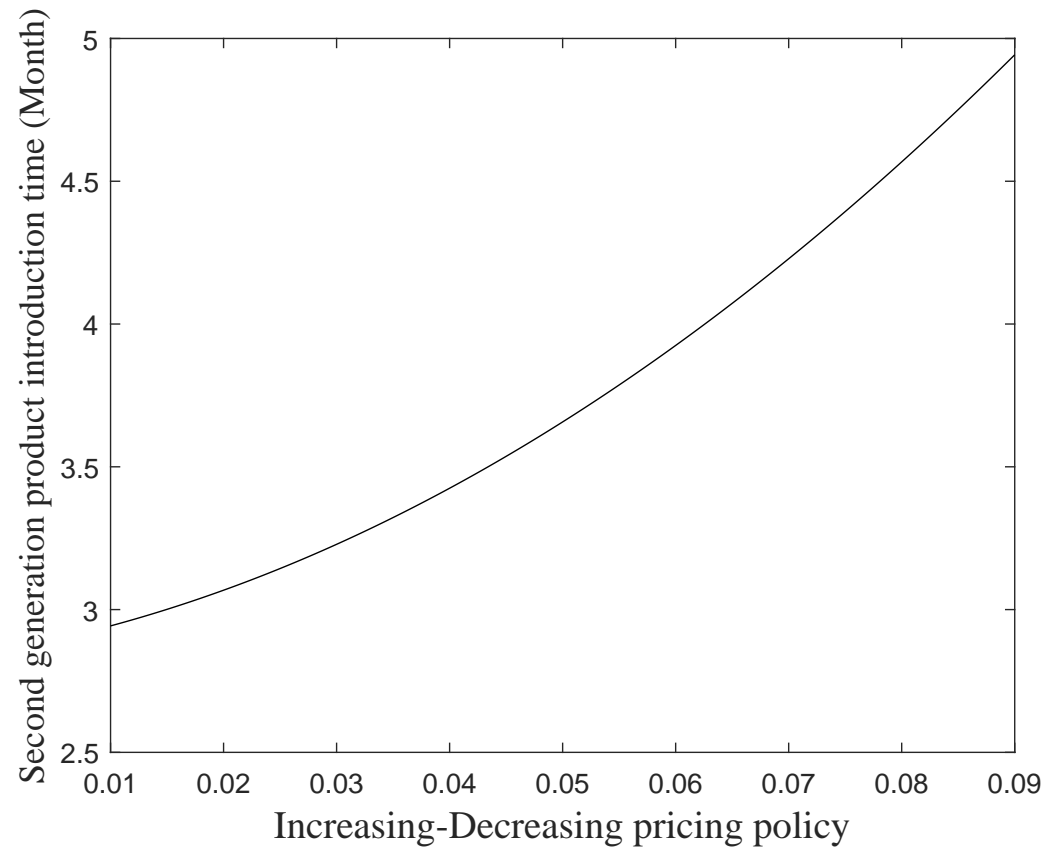

Figure 6.17: Second-generation product introduction time with Approach (II) (IncreasingDecreasing pricing policy-phase out transition) 


\subsection{Cross functional advertising}

All the sensitivity analyses in this chapter assumed $10 \%$ of a product's price sales for advertising expenses. In order to investigate how different advertising budget percentage affect the NPV, I used cross functional advertising analysis. In this section, we investigate decreasing pricing and increasing-decreasing pricing policy when the advertising budget is changing from $0 \%$ to $20 \%$ of price sales. For the first product, always decreasing pricing policy is considered while for the second product either decreasing or increasing-decreasing pricing policy is considered.

In Figure 6.18, $t=0$ shows the result of Scenario 3, phase out transition-simultaneous strategy, and $t=1$ to $t=7$ show the results of Scenario 2, phase out transition-sequential strategy. Figure 6.19 shows the decreasing pricing policy when the price changes only the drift rate. When the advertising budget percentage rate changes from $0 \%$ to $20 \%$, the secondgeneration product introduction time remains the same at month 2.5. In this scenario, the NPV reaches a peak value of $\$ 2.2$ million with $15 \%$ of advertising budget. In this scenario, the second-generation product has to be introduced as early as the first-generation product introduction time due to increasing demand for the second-generation product. Figure 6.22 shows the maximum of advertising budget percentage rates at $0 \%, 5 \%, 10 \%, 15 \%$, and $20 \%$. 


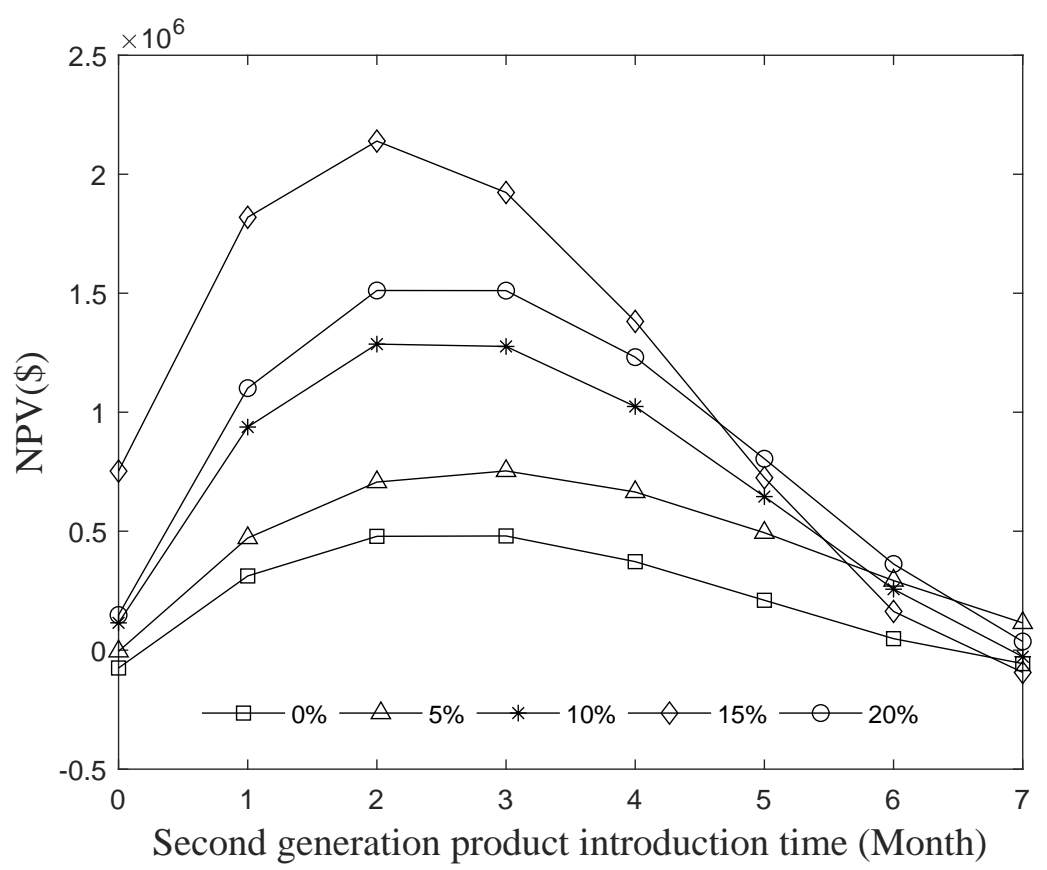

Figure 6.18: Second-generation product introduction time when advertising budget percentage changes (Decreasing pricing policy-phase out transition)

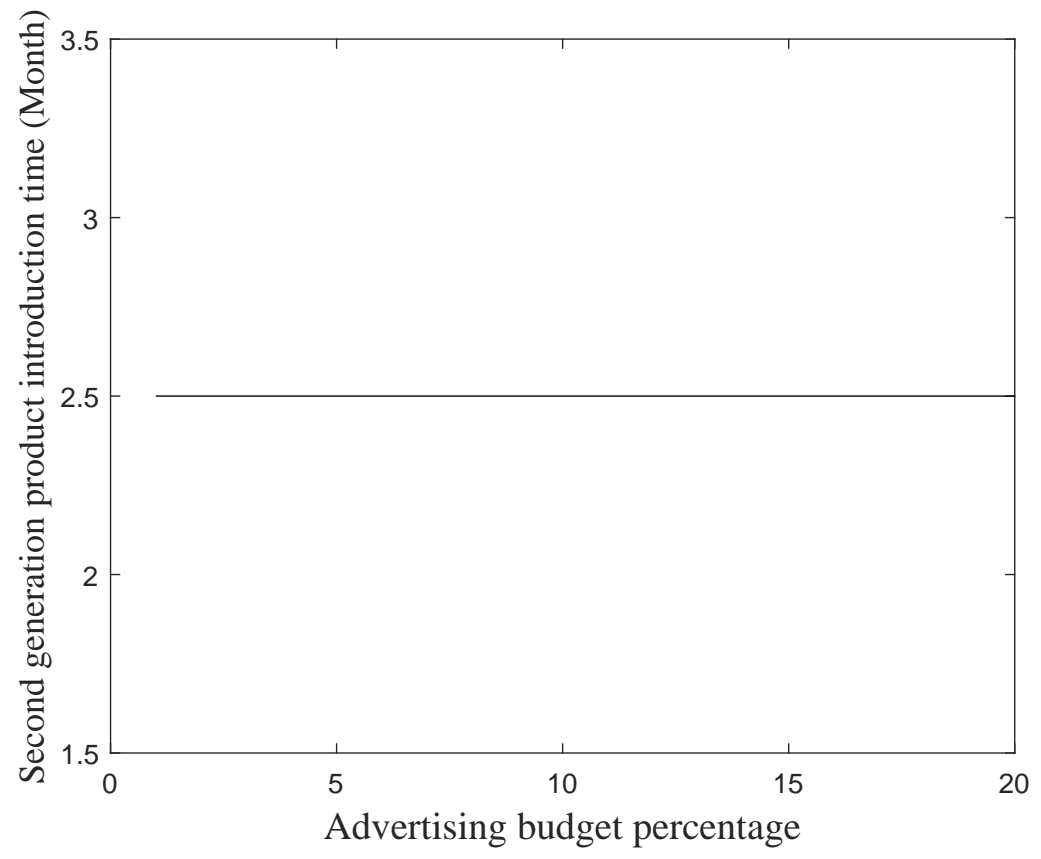

Figure 6.19: Second-generation product introduction time when advertising budget percentage changes (Decreasing pricing policy-phase out transition)

In Figure $6.20, t=0$ shows the result of Scenario 3, phase out transition-simultaneous 
strategy, and $t=1$ to $t=8$ show the results of Scenario 2 , phase out transition-sequential strategy. Figure 6.20 shows the increasing-decreasing pricing policy when the price changes only the drift rate. In this scenario, when the advertisement budget percentage is zero, NPV reaches a peak value at $\$ 300$ million. When the advertisement budget percentage is $15 \%$ NPV reaches a peak value at $\$ 600$ million. Figure 6.21 shows when the advertising percentage budget rate increases from $0 \%$ to $20 \%$, the second-generation product introduction time is shifted to the left. This means the second generation product should be introduced earlier. In this scenario, when the advertisement budget percentage is zero, the second generation product introduction time is at month 6 and when the advertisement budget percentage is $15 \%$, the second-generation product introduction time is at month 5. Figure 6.23 shows the maximum of advertising budget percentage rates at $0 \%, 5 \%, 10 \%, 15 \%$, and $20 \%$.

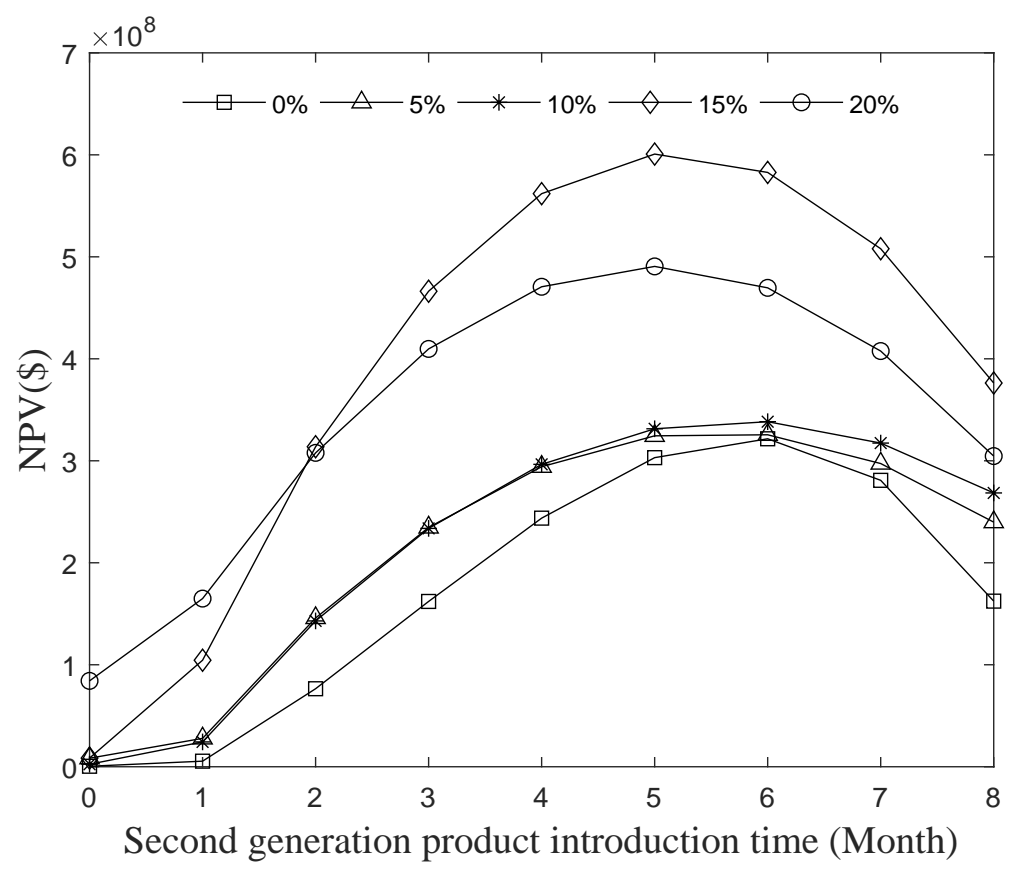

Figure 6.20: Second-generation product introduction time when advertising budget percentage changes (Increasing-Decreasing pricing policy-phase out transition) 


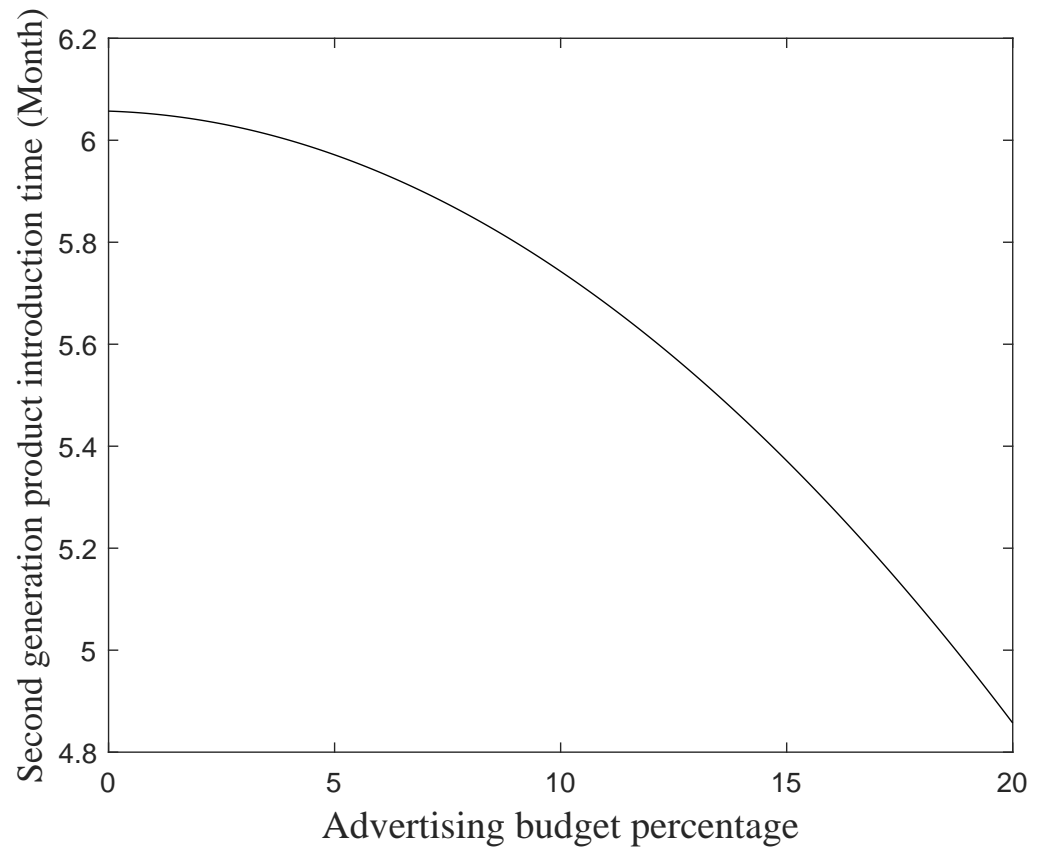

Figure 6.21: Second-generation product introduction time when advertising budget percentage changes (Increasing-Decreasing pricing policy-phase out transition)

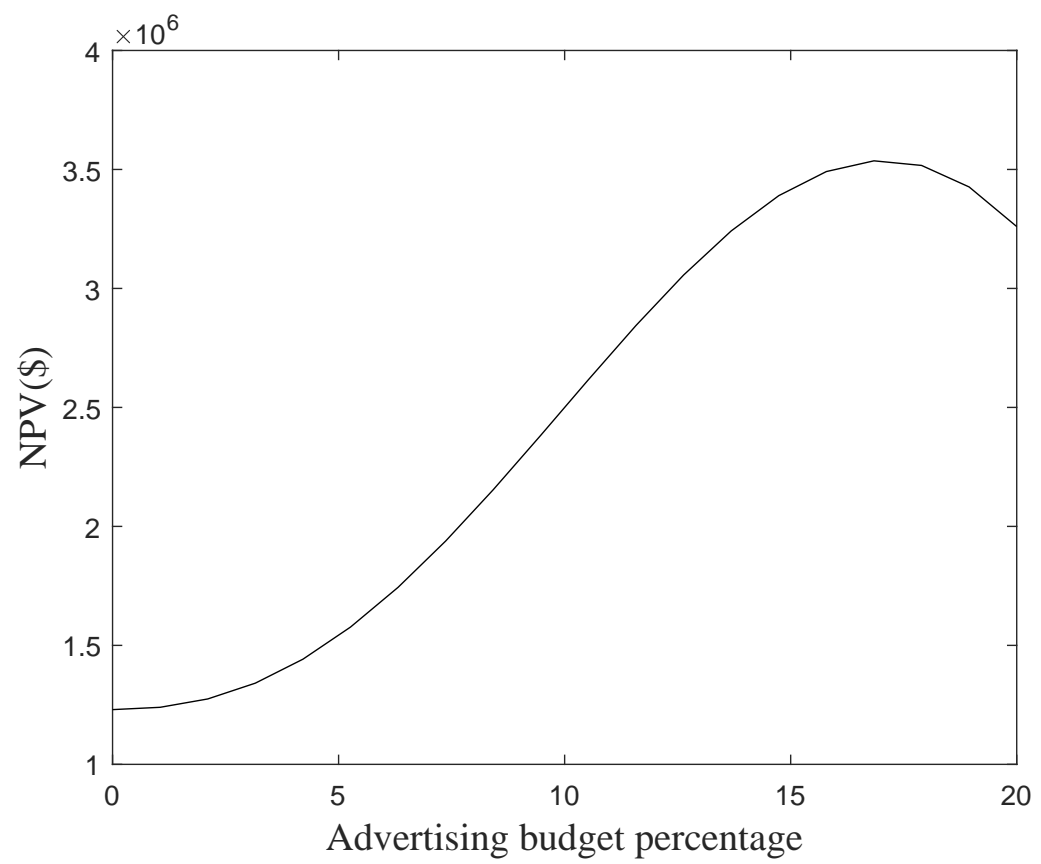

Figure 6.22: Advertising budget percentage changes (Decreasing pricing policy-phase out transition) 


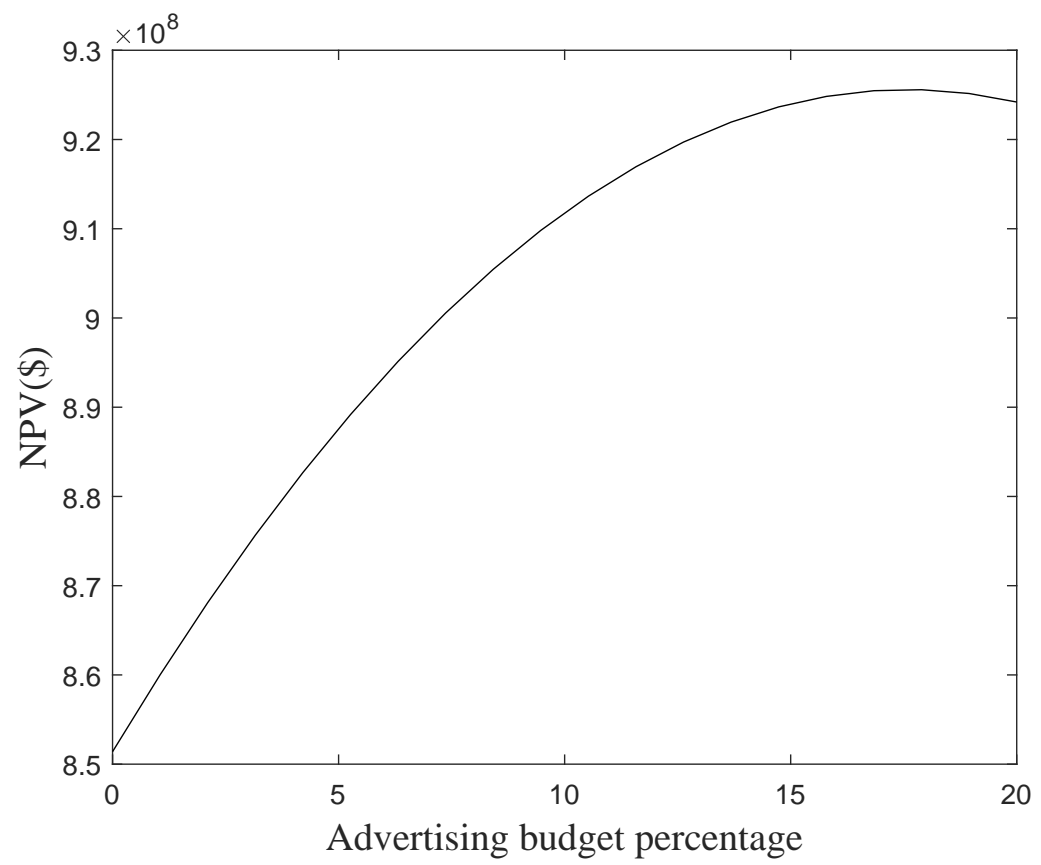

Figure 6.23: Advertising budget percentage changes (Increasing-Decreasing pricing policyphase out transition)

\subsection{Comparing the results of Chapter 5 and Chapter 6}

Section 5.5 investigated three different scenarios and two different pricing policies in two different approaches. Section 6.4 investigated three different scenarios, two different pricing policies, and percentage of budgeting advertisement in two different approaches. Decreasing and increasing-decreasing pricing policies are two different pricing policies that are considered in this research. When price changes only the drift rate and price changes both drift rate and volatility are two approaches that are investigated in this research. Comparing Sections 5.5 and 6.4 in Scenario 1, total replacement, and decreasing pricing policy shows that when invested in advertisement, NPV increases and also second-generation product introduction time will be earlier. Comparing Sections 5.5 and 6.4 in Scenario 2 and Scenario 3, phase out transition-simultaneous and phase out transition-sequential strategy, decreasing pricing 
policy in both approaches shows that when we have an advertisement, the NPV decreases and also second-generation product has to be introduced as early as possible. Comparing the Sections 5.5 and 6.4 in Scenario 2 and Scenario 3, phase out transition-simultaneous and phase out transition-sequential strategy, increasing-decreasing pricing policy in both approaches shows that when we have the advertisement, the NPV increases and also secondgeneration PIT is delayed. 


\subsection{Summary}

The advertising effect on PIT while incorporating timing and pricing effects has been studied in this chapter. Growth and decaying regimes for each product are defined by a GBM. Diffusion adoption function is used to estimate the demand parameters including drift rate, volatility, and correlation. Dynamic programming in the lattice is utilized to optimize NPV in a given period. Two different pricing policies in three different scenarios, as explained in section 3.2 in two different approaches, are used for sensitive analysis. Decreasing and increasing-decreasing pricing policies are two types of pricing policies that are considered in this chapter. Furthermore, there are two different approaches used for changing the price of a product. One approach looks at the drift rate and the second approach looks at the both drift rate and volatility. In all of these sensitive analyses, the advertising budget percentage is considered to be $10 \%$ of price sales. Results show that when decreasing pricing policy is used, the second-generation product has to be introduced as early as the first-generation product. When the increasing-decreasing pricing policy is used, as the decreasing pricing rate increases from $-70 \%$ to $-30 \%$, the second-generation PIT is delayed. Also, in increasingdecreasing pricing policy, as the increasing pricing rate increases from 0.01 to 0.09 , the second-generation PIT is delayed. When the advertising budget percentage increases, the NPV increases up to a certain level and then it will be saturated. 


\section{Chapter 7}

\section{Conclusion and Future work}

Product introduction time is a crucial decision for many companies, specially when the upcoming product is the extension of the first-generation product. This research has investigated different product introduction time strategies such as sequential and simultaneous. Furthermore, total replacement-sequential strategy, phase out transition-sequential strategy, and phase out transition-simultaneous strategy are three different scenarios that have been investigated in this research. In order to investigate such strategies comprehensively, it is important to capture different aspects such as product life cycle (PLC) and different pricing and advertising policies.

In this research, simultaneous and sequential product introduction time strategies were investigated. To this end, two generations of products were considered in this research, while

demand for each product consists of two regimes life cycle. Each regime was defined by a geometric Brownian motion (GBM). Demand parameters including drift rate, volatility, and correlation in each GBM were estimated by the Bass model. Also, there is a correlation between different regimes for different products. A lattice approach was used to discretize the correlated GBMs. The flexibility was used as a competitive strategy to overcome the market uncertainty. Flexibilities, such as expansion, contraction, and switching, were identified between two generations of products in this research. A dynamic programming method was utilized to maximize the NPV over a given period. 
In the current competitive market, it is paramount for manufacturing companies to apply practical models that can help with finding the best possible time for introducing their new products to the market. Accordingly, product introduction time for a new generation product can have a significant effect on the first-generation product. Thus, from a managerial perspective, it is important to find the best second-generation product introduction time such that the net present value for both products can be maximized. Furthermore, from a management point of view, it is also important to understand the optimal interval between successive products that can help the design/development team so that upcoming generation is ready on time. In this light, it will be very important for decision-makers to set a target goal for the existing product and introduce the new generation product in the marketplace when the desired goal is achieved. This will definitely help to maximize the net present value of such investment. From the firm's perspective, knowledge regarding introduction of a new product in the near future and the technological substitution and market expansion effects created by it can be expected to have a significant impact on the strategy pursued by the firm with regard to these products. Thus, knowing the best product introduction time, firms can meaningfully reduce their costs (e.g., inventory cost), which will ultimately help them to get the competitive edge. Drawing on the above, models developed in this research focus on finding the optimal product introduction time that can help the decision-makers in any manufacturing company (in any industry) to maximize the net present value of their investments. For example, when Apple Company decides to introduce a new generation of Apple iPhone, the model developed in this research can be used to help with finding the best new generation product introduction time. Additionally, models developed in this research can also be used to help with optimizing the marketing and developing process involved in planning for a new generation product. The application of models developed in this research can also be extended to many other industries such as pharmaceutical and semiconductors companies.

The first objective of this research aims to find the optimum product introduction time for the second-generation product with consideration of the PLC. The first model investi- 
gated both sequential and simultaneous timing strategies to determine the optimum secondgeneration product introduction time by maximizing the NPV over a given period of time. Results of the first model show that as the drift rate, volatility, and initial demand for a firstgeneration product increase, it is expected that the demand for the first-generation product will increase. As a result, the second-generation product introduction time has to be delayed. While the unit cost of the first-generation product increases, lower NPV is expected, and hence the second-generation product has to be introduced earlier.

The second objective of this research aims to find the optimum product introduction time for the second-generation product for different pricing policies by maximizing the NPV over a given period of time. In the second model, the effects of PLC and pricing on the second-generation product introduction time for simultaneous and sequential strategies are investigated. As the demand changes over time, pricing of a product will be affected. Results demonstrate that in decreasing pricing policy, the second-generation product has to be introduced as early as the first-generation product. In increasing-decreasing pricing policy, as the decreasing pricing rate increases the second-generation product introduction time is delayed. Furthermore, in increasing-decreasing pricing policy, as the increasing pricing rate increases, the second-generation product introduction time is delayed.

The third objective of this research aims to find the optimum product introduction time for the second-generation product for a generic advertising policy within a certain level of advertising budget by maximizing the NPV over a given period of time. This model was developed to capture the effects of PLC, pricing, and advertising. As the demand of the first-generation product affects the demand of the second-generation product or vise-versa, it makes the advertising policy complicated. Hence, by addressing PLC, pricing, and advertising in this model, all of these important factors are considered. For sensitivity analyses, the advertising budget percentage is considered to be $10 \%$ of price sales. Results show that when decreasing pricing policy is used, the second-generation product has to be introduced as early as the first-generation product. When the increasing-decreasing pricing policy is used, the second-generation PIT is delayed as the decreasing pricing rate is increasing. Further- 
more, in the increasing-decreasing pricing policy, as the increasing pricing rate is increasing, the second-generation PIT is delayed. When the advertising budget percentage increases, the NPV increases up to a certain level and then it will be saturated.

\subsection{Research contributions}

The contributions of this research are five-fold as follows. First, developing an integrated methodology that incorporates aspects of both marketing and manufacturing systems is investigated in this research. The existing literature has studied PIT only from marketing points of view. Decision makers in a company are able to consider product sales as well as manufacturing aspects to maximize company profit by integrating these two aspects. Second, the framework proposed in a coherent decision-making methodology for sequential products that will maximize NPV over a given period while PLC, pricing, and advertising are taken into account. Third, the proposed models have the ability of incorporating the flexibility of the system for determining the optimal PIT. By using a flexible system, decision makers in manufacturing may share the production capacity between two products. Having such an integrated system can cover the uncertainty demand over time. Fourth, the production cost is usually overlooked in studies concerned with sequential strategy. However, the developed model in this research considers production cost as a major contributing parameter. This gives the proposed model the ability to handle case studies that have not been dealt with

in the existing literature. Finally, the value of the PIT is expressed in terms of dollar value and this would help managers to make the decision easily. 


\subsection{Challenges, limitations and future work}

Like any research endeavor, this research faces challenges and limitations. In this particular research, I considered 5 years given period, 60 steps, and each step has a decision making process. As the number of steps are increased, the computation time will be dramatically increased. A server consisting of 24 Intel Xeon CPU E5-2650 v4, 2.20 GHz with 256 GB RAM is used to run the code associated with each model. Running such a code is time-consuming and requires higher computational power. It is expected that information technology development in the future will design computer systems with faster processing time which will definitely lower the computation time.

As discussed in Chapter 3, each product could be in a growth regime or a decaying regime where each regime is defined by a GBM. In order to determine drift rate, volatility, and correlation of each product, the Bass model is utilized. The Bass parameters such as $p, q$, and $m$ have an effect on the PLC, which represents the unit sales curve for durable products, from the time it is introduced in the market until it is removed from the market. When a company introduces a similar product, the potential buyers could turn to buy the new product, and hence the demand for the current product could go down. This means that the PLC of the current product may be negatively affected. There are factors that could affect $p$, which is innovation coefficient. Innovators are those whose decision to adopt a product is independent of the decision of the others. For example, potential buyers' income has a significant effect on parameter $p$. Accordingly, a potential buyer with a higher income can afford to buy a product easier than those with lower income. As a result, these early buyers have a positive effect on the PLC. In light of this, it is necessary to emphasize that the models developed in this research did not consider the impact of some important non-marketing factors such as changes in potential buyers' income.

In the competitive market, other competitors could affect the PLC by introducing their own new product. Competitors' pricing policies can also affect PIT. A model that incorporates a duopoly firm with two generations of product and includes the effects of competition 
is required to investigates the changes PLC may have as a result of a new brand entry by another competitor in the market. That would be another line of research for the potential future work.

No research has practically studied the effects of marketing mix variables such as pricing, advertising and non-marketing factor, such as potential buyers' income, simultaneously. Therefore, consideration of these variables is recommended as possible future research.

The period which has been considered to value the PIT strategy is important and depends on the uncertain demands for each product and cost. This research considered only a fixed period. However, considering the random termination of a project by defining a probability distribution function as a function of cumulative demand could enable more flexibility in the system.

This research considered a two-regime life cycle (growth regime and decay regime) for each product, in which each regime is defined by a GBM. A three-regime life cycle (growth regime, maturity regime, and decay regime) or four-regime life cycle could be an extension of the current research. In a three-regime life cycle, the first regime of the PLC is a growth regime then, it switches to the maturity regime and it ends up to a decaying regime. 


\section{Appendix A}

\section{When price changes the drift rate}

$$
F=\theta e^{\mu h+X(t)} .
$$

Where $X(t)$, price function, is a function of time.

Using Ito's Lemma (Hull, 2009):

$$
d F=\left(\frac{\partial F}{\partial \theta} \mu \theta+\frac{\partial F}{\partial t}+\frac{1}{2} \frac{\partial^{2} F}{\partial \theta^{2}} \sigma^{2} \theta^{2}\right) d t+\frac{\partial F}{\partial \theta} \sigma \theta d z
$$

after substitution

$$
d F=\left[\mu+X^{\prime}(t)\right] F d t+\sigma F d z .
$$

Correlated demand for products $A$ and $B$ in combined regime $\left(A_{g}, B_{g}\right)$

$$
\begin{aligned}
& d \theta_{g}^{A}=\left[\mu_{g}^{A}+X^{\prime}(t)\right] \theta_{g}^{A} d t+\sigma_{g}^{A} \theta_{g}^{A} d Z_{g}^{A}, \\
& d \theta_{g}^{B}=\left[\mu_{g}^{B}+X^{\prime}(t)\right] \theta_{g}^{B} d t+\sigma_{g}^{B} \theta_{g}^{B} d Z_{g}^{B} .
\end{aligned}
$$


According Equation (A.3) we have

$$
\begin{gathered}
d F=\left[\mu+X^{\prime}(t)\right] F d t+\sigma F d z, \\
g(t, F)=\ln (F),
\end{gathered}
$$

Differentiate $g(t, F)$

$$
\begin{gathered}
d g=\frac{\partial g}{\partial t} d t+\frac{\partial g}{\partial F} d F+\frac{\partial^{2} g}{\partial F^{2}} d F^{2} . \\
d \ln F=\left[\mu-\frac{1}{2} \sigma^{2}+X^{\prime}(t)\right] d t+\sigma_{g}^{A} d z_{g}^{A} .
\end{gathered}
$$

For products $A$ and $B$ using Appendix D

$$
\begin{aligned}
& d \ln \theta_{g}^{A}=\left[\mu_{g}^{A}-\frac{1}{2} \sigma_{g}^{2 A}+X^{\prime}(t)\right] d t+\sigma_{g}^{A} d z_{g}^{A}, \\
& d \ln \theta_{g}^{B}=\left[\mu_{g}^{B}-\frac{1}{2} \sigma_{g}^{2^{B}}+X^{\prime}(t)\right] d t+\sigma_{g}^{B} d z_{g}^{B} .
\end{aligned}
$$

According two above equations and using method in Appendix D, eight uncorrelated processes are obtained.

$$
\begin{aligned}
d \Psi_{1} & =\left(\left[\mu_{g}^{A}-\frac{1}{2} \sigma_{g}^{2_{g}^{A}}+X^{\prime}(t)\right] \sigma_{g}^{B}+\left[\mu_{g}^{B}-\frac{1}{2}{\sigma^{2}}_{g}^{B}+X^{\prime}(t)\right] \sigma_{g}^{A}\right) d t \\
& +\sigma_{g}^{A} \sigma_{g}^{B} \sqrt{2\left(1+\rho_{g g}\right)} d z \Psi_{1},
\end{aligned}
$$




$$
\begin{aligned}
d \Psi_{2} & =\left(\left[\mu_{g}^{A}-\frac{1}{2} \sigma_{g}^{2 A}+X^{\prime}(t)\right] \sigma_{g}^{B}-\left[\mu_{g}^{B}-\frac{1}{2} \sigma_{g}^{2 B}+X^{\prime}(t)\right] \sigma_{g}^{A}\right) d t \\
& +\sigma_{g}^{A} \sigma_{g}^{B} \sqrt{2\left(1-\rho_{g g}\right)} d z \Psi_{2} .
\end{aligned}
$$

Similarly, for combined regime $\left(A_{d}, B_{g}\right)$

$$
\begin{aligned}
d \Psi_{3} & =\left(\left[\mu_{d}^{A}-\frac{1}{2} \sigma_{d}^{2 A}+X^{\prime}(t)\right] \sigma_{g}^{B}+\left[\mu_{g}^{B}-\frac{1}{2}{\sigma^{2}}_{g}^{B}+X^{\prime}(t)\right] \sigma_{d}^{A}\right) d t \\
& +\sigma_{d}^{A} \sigma_{g}^{B} \sqrt{2\left(1+\rho_{d g}\right)} d z \Psi_{3}, \\
d \Psi_{4} & =\left(\left[\mu_{d}^{A}-\frac{1}{2} \sigma_{d}^{2 A}+X^{\prime}(t)\right] \sigma_{g}^{B}-\left[\mu_{g}^{B}-\frac{1}{2} \sigma_{g}^{2^{B}}+X^{\prime}(t)\right] \sigma_{d}^{A}\right) d t \\
& +\sigma_{d}^{A} \sigma_{g}^{B} \sqrt{2\left(1-\rho_{d g}\right)} d z \Psi_{4} .
\end{aligned}
$$

For combined regime $\left(A_{g}, B_{d}\right)$

$$
\begin{aligned}
d \Psi_{5} & =\left(\left[\mu_{g}^{A}-\frac{1}{2} \sigma_{g}^{2 A}+X^{\prime}(t)\right] \sigma_{d}^{B}+\left[\mu_{d}^{B}-\frac{1}{2}{\sigma^{2}}_{d}^{B}+X^{\prime}(t)\right] \sigma_{g}^{A}\right) d t \\
& +\sigma_{g}^{A} \sigma_{d}^{B} \sqrt{2\left(1+\rho_{g d}\right)} d z \Psi_{5}, \\
d \Psi_{6} & =\left(\left[\mu_{d}^{A}-\frac{1}{2} \sigma_{d}^{2 A}+X^{\prime}(t)\right] \sigma_{d}^{B}-\left[\mu_{g}^{B}-\frac{1}{2} \sigma_{g}^{2^{B}}+X^{\prime}(t)\right] \sigma_{d}^{A}\right) d t \\
& +\sigma_{d}^{A} \sigma_{g}^{B} \sqrt{2\left(1-\rho_{g d}\right)} d z \Psi_{6} .
\end{aligned}
$$

Also, for combined regime $\left(A_{d}, B_{d}\right)$ we have:

$$
\begin{aligned}
d \Psi_{7} & =\left(\left[\mu_{d}^{A}-\frac{1}{2} \sigma_{d}^{2 A}+X^{\prime}(t)\right] \sigma_{d}^{B}+\left[\mu_{d}^{B}-\frac{1}{2} \sigma_{d}^{2}{ }_{d}^{B}+X^{\prime}(t)\right] \sigma_{d}^{A}\right) d t \\
& +\sigma_{d}^{A} \sigma_{d}^{B} \sqrt{2\left(1+\rho_{d d}\right)} d z \Psi_{7}
\end{aligned}
$$




$$
\begin{aligned}
d \Psi_{8} & =\left(\left[\mu_{d}^{A}-\frac{1}{2} \sigma_{d}^{2 A}+X^{\prime}(t)\right] \sigma_{d}^{B}-\left[\mu_{d}^{B}-\frac{1}{2} \sigma_{d}^{2^{B}}+X^{\prime}(t)\right] \sigma_{d}^{A}\right) d t \\
& +\sigma_{d}^{A} \sigma_{d}^{B} \sqrt{2\left(1-\rho_{d d}\right)} d z \Psi_{8} .
\end{aligned}
$$




\section{Appendix B}

\section{When price changes both volatility and drift rate}

$$
F=e^{\mu h+\theta X(t)}
$$

Where $X(t)$, price function, is a function of time.

Using Ito's Lemma:

$$
d F=\left(\frac{\partial F}{\partial \theta} \mu \theta+\frac{\partial F}{\partial t}+\frac{1}{2} \frac{\partial^{2} F}{\partial \theta^{2}} \sigma^{2} \theta^{2}\right) d t+\frac{\partial F}{\partial \theta} \sigma \theta d z
$$

after substitution

$$
d F=\left[\mu X(t)+X^{\prime}(t)+\frac{X^{2}(t)}{2} \sigma^{2}\right] F d t+X(t) \sigma F d z
$$

Correlated demand for products $A$ and $B$ in combined regime $\left(A_{g}, B_{g}\right)$

$$
d \theta_{g}^{A}=\left[\mu_{g}^{A} X(t)+X^{\prime}(t)+\frac{X^{2}(t)}{2} \sigma_{g}^{2 A}\right] \theta_{g}^{A} d t+X(t) \sigma_{g}^{A} \theta_{g}^{A} d Z_{g}^{A}
$$




$$
d \theta_{g}^{B}=\left[\mu_{g}^{B} X(t)+X^{\prime}(t)+\frac{X^{2}(t)}{2} \sigma_{g}^{2 B}\right] \theta_{g}^{B} d t+X(t) \sigma_{g}^{B} \theta_{g}^{B} d Z_{g}^{B}
$$

According Equation (B.3) we have

$$
\begin{gathered}
d F=\left[\mu X(t)+X^{\prime}(t)+\frac{X^{2}(t)}{2} \sigma^{2}\right] F d t+X(t) \sigma F d z \\
g(t, F)=\ln (F),
\end{gathered}
$$

Differentiate $g(t, F)$

$$
\begin{gathered}
d g=\frac{\partial g}{\partial t} d t+\frac{\partial g}{\partial F} d F+\frac{\partial^{2} g}{\partial F^{2}} d F^{2}, \\
d \ln F=\left[\mu X(t)+X^{\prime}(t)\right] d t+\sigma X(t) d z_{g}^{A} .
\end{gathered}
$$

For products $A$ and $B$ using Appendix D

$$
\begin{aligned}
& d \ln \theta_{g}^{A}=\left[\mu_{g}^{A} X(t)+X^{\prime}(t)\right] d t+\sigma_{g}^{A} X(t) d z_{g}^{A}, \\
& d \ln \theta_{g}^{B}=\left[\mu_{g}^{B} X(t)+X^{\prime}(t)\right] d t+\sigma_{g}^{B} X(t) d z_{g}^{B} .
\end{aligned}
$$

According two above equations and using method in Appendix D, eight uncorrelated processes are obtained.

$$
\begin{aligned}
d \Psi_{1} & =\left[\left(X(t)\left[\mu_{g}^{A} X(t)+X^{\prime}(t)\right] \sigma_{g}^{B}\right)\right]+\left[\left(X(t)\left[\mu_{g}^{B} X(t)+X^{\prime}(t)\right] \sigma_{g}^{A}\right)\right] d t \\
& +X^{2}(t) \sigma_{g}^{A} \sigma_{g}^{B} \sqrt{2\left(1+\rho_{g g}\right)} d z \Psi_{1},
\end{aligned}
$$




$$
\begin{aligned}
d \Psi_{2} & =\left[\left(X(t)\left[\mu_{g}^{A} X(t)+X^{\prime}(t)\right] \sigma_{g}^{B}\right)\right]-\left[\left(X(t)\left[\mu_{g}^{B} X(t)+X^{\prime}(t)\right] \sigma_{g}^{A}\right)\right] d t \\
& +X^{2}(t) \sigma_{g}^{A} \sigma_{g}^{B} \sqrt{2\left(1-\rho_{g g}\right)} d z \Psi_{2} .
\end{aligned}
$$

Similarly, for combined regime $\left(A_{d}, B_{g}\right)$

$$
\begin{aligned}
d \Psi_{3} & =\left[\left(X(t)\left[\mu_{d}^{A} X(t)+X^{\prime}(t)\right] \sigma_{g}^{B}\right)\right]+\left[\left(X(t)\left[\mu_{g}^{B} X(t)+X^{\prime}(t)\right] \sigma_{d}^{A}\right)\right] d t \\
& +X^{2}(t) \sigma_{d}^{A} \sigma_{g}^{B} \sqrt{2\left(1+\rho_{g g}\right)} d z \Psi_{3}, \\
d \Psi_{4} & =\left[\left(X(t)\left[\mu_{d}^{A} X(t)+X^{\prime}(t)\right] \sigma_{g}^{B}\right)\right]-\left[\left(X(t)\left[\mu_{g}^{B} X(t)+X^{\prime}(t)\right] \sigma_{d}^{A}\right)\right] d t \\
& +X^{2}(t) \sigma_{d}^{A} \sigma_{g}^{B} \sqrt{2\left(1-\rho_{g g}\right)} d z \Psi_{4} . \\
& \\
d \Psi_{5} & =\left[\left(X(t)\left[\mu_{g}^{A} X(t)+X^{\prime}(t)\right] \sigma_{d}^{B}\right)\right]+\left[\left(X(t)\left[\mu_{d}^{B} X(t)+X^{\prime}(t)\right] \sigma_{g}^{A}\right)\right] d t \\
& +X^{2}(t) \sigma_{g}^{A} \sigma_{d}^{B} \sqrt{2\left(1+\rho_{g g}\right)} d z \Psi_{5}, \\
d \Psi_{6} & =\left[\left(X(t)\left[\mu_{g}^{A} X(t)+X^{\prime}(t)\right] \sigma_{d}^{B}\right)\right]-\left[\left(X(t)\left[\mu_{d}^{B} X(t)+X^{\prime}(t)\right] \sigma_{g}^{A}\right)\right] d t \\
& +X^{2}(t) \sigma_{g}^{A} \sigma_{d}^{B} \sqrt{2\left(1-\rho_{g g}\right)} d z \Psi_{6} .
\end{aligned}
$$

Also, for combined regime $\left(A_{d}, B_{d}\right)$

$$
\begin{aligned}
d \Psi_{7} & =\left[\left(X(t)\left[\mu_{d}^{A} X(t)+X^{\prime}(t)\right] \sigma_{d}^{B}\right)\right]+\left[\left(X(t)\left[\mu_{d}^{B} X(t)+X^{\prime}(t)\right] \sigma_{d}^{A}\right)\right] d t \\
& +X^{2}(t) \sigma_{d}^{A} \sigma_{d}^{B} \sqrt{2\left(1+\rho_{g g}\right)} d z \Psi_{7},
\end{aligned}
$$




$$
\begin{aligned}
d \Psi_{8} & =\left[\left(X(t)\left[\mu_{d}^{A} X(t)+X^{\prime}(t)\right] \sigma_{d}^{B}\right)\right]-\left[\left(X(t)\left[\mu_{d}^{B} X(t)+X^{\prime}(t)\right] \sigma_{d}^{A}\right)\right] d t \\
& +X^{2}(t) \sigma_{d}^{A} \sigma_{d}^{B} \sqrt{2\left(1-\rho_{g g}\right)} d z \Psi_{8} .
\end{aligned}
$$




\section{Appendix $\mathrm{C}$}

\section{Step size and Branch Probabilities by}

\section{Matching the First and Second}

\section{Moments}

Assume that the $d x=\mu d t+\sigma d B$ is a geometric Brownian motion. $(\phi)$ and $(-\phi)$ are the up and down movement step size, respectively.

Equations (C.1) , and (C.2) shows the first and the second moment matching.

$$
\begin{array}{r}
\pi(\phi)+(1-\pi)(-\phi)=\mu h, \\
\pi(\phi)^{2}+(1-\pi)(-\phi)^{2}-\mu^{2}(h)^{2}=\sigma^{2} h .
\end{array}
$$

Where $\pi$ is the probability to move up ,and $(1-\pi)$ is the probability to move down. 
$\pi$ can be found from Equation (C.1)

$$
\begin{aligned}
\pi(\phi)+(1-\pi)(-\phi) & =\mu h, \\
\pi \phi-\phi+\pi \phi & =\mu h, \\
2 \pi \phi & =\phi+\mu h, \\
\pi & =\frac{\phi+\mu h}{2 \phi}, \\
\pi & =\frac{1}{2}+\frac{\mu h}{2 \phi} .
\end{aligned}
$$

After substitution Equation (C.7) into Equation (C.2):

$$
\begin{aligned}
\frac{\phi+\mu h}{2 \phi} \times(\phi)^{2}+\left[1-\frac{\phi+\mu h}{2 \phi}\right] \times(\phi)^{2} & =\mu^{2}(h)^{2}+\sigma^{2} h, \\
\frac{(\phi+\mu h) \phi}{2}+\frac{(2 \phi-\phi-\mu h) \phi}{2} & =\mu^{2}(h)^{2}+\sigma^{2} h, \\
\frac{(\phi+\mu h) \phi}{2}+\frac{2}{2} \phi^{2}-\frac{(\phi+\mu h) \phi}{2} & =\mu^{2}(h)^{2}+\sigma^{2} h, \\
\phi^{2} & =\mu^{2}(h)^{2}+\sigma^{2} h, \\
\phi & =\sqrt{\mu^{2}(h)^{2}+\sigma^{2} h .}
\end{aligned}
$$




\section{Appendix D}

\section{Uncorrelating the correlated processes}

If $c_{1}$ and $c_{2}$ are two correlated processes and given as follow:

$$
\begin{aligned}
& d c_{1}=a_{1} d t+b_{1} d z_{1}, \\
& d c_{2}=a_{2} d t+b_{2} d z_{2} .
\end{aligned}
$$

Where $a_{1}, a_{2}, b_{1}$, and $b_{2}$ are constants and $d z_{1}$ and $d z_{2}$ are the correlated Wiener processes with a correlation $\rho$ factor. The two new uncorrelated variables

$$
\begin{aligned}
& e_{1}=b_{2} c_{1}+b_{1} c_{2}, \\
& e_{2}=b_{2} c_{1}-b_{1} c_{2},
\end{aligned}
$$

can be defines as following equations:

$$
\begin{aligned}
& d e_{1}=\left(a_{1} b_{2}+a_{2} b_{1}\right) d t+b_{1} b_{2} \sqrt{2(1+\rho)} d z_{3}, \\
& d e_{2}=\left(a_{1} b_{2}-a_{2} b_{1}\right) d t+b_{1} b_{2} \sqrt{2(1-\rho)} d z_{4} .
\end{aligned}
$$

$a_{1} b_{2}+a_{2} b_{1}$, and $a_{1} b_{2}-a_{2} b_{1}$ are the instantaneous means of $d e_{1}$, and $d e_{2}$, respectively. $b_{1} b_{2} \sqrt{2(1+\rho)}$ and $b_{1} b_{2} \sqrt{2(1-\rho)}$ are the volatilities of $d e_{1}$ and $d e_{2}$, respectively. $d z_{3}$, and 
$d z_{4}$ are the uncorrelated Wiener processes. 


\section{References}

Adusumilli, N., Davis, S., and Fromme, D. (2016). Economic evaluation of using surge valves in furrow irrigation of row crops in louisiana: A net present value approach. Agricultural Water Management, 17(10), 61-65.

Albey, E., Bilge, Ü., and Uzsoy, R. (2017). Multi-dimensional clearing functions for aggregate capacity modelling in multi-stage production systems. International Journal of Production Research, 55(14), 4164-4179.

Ashuri, B., Kashani, H., Molenaar, K., Lee, S., and Lu, J. (2011). Risk-neutral pricing approach for evaluating bot highway projects with government minimum revenue guarantee options. Journal of Construction Engineering and Management, 138(4), 545-557.

Babazadeh, R., Jolai, F., and Razmi, J. (2015). Developing scenario-based robust optimisation approaches for the reverse logistics network design problem under uncertain environments. International Journal of Services and Operations Management, 20(4), 418-440.

Babich, V. (2006). Vulnerable options in supply chains: Effects of supplier competition. Naval Research Logistics, 53(7), 656-673.

Bass, F. M. (1969). A new product growth model for consumer durables. Management Science, 15(5), 215-227.

Bass, F. M., Krishnan, T. V., and Jain, D. C. (1994). Why the Bass model fits without decision variables. Marketing Science, 13(3), 203-223. 
Bass, F. M., Jain, D., and Krishnan, T. (2000). Modeling the marketing-mix influence in new-product diffusion. Kluwer Academic Publishers: Boston, MA.

Bass, F. M., Krishnamoorthy, A., Prasad, A., and Sethi, S. P. (2005). Generic and brand advertising strategies in a dynamic duopoly. Marketing Science, 24(4), 556-568.

Bayus, B. L. (1992). The dynamic pricing of next generation consumer durables. Marketing Science, 11(3), 251-265.

Bayus, B. L., Jain, S., and Rao, A. G. (1997). Too little, too early: Introduction timing and new product performance in the personal digital assistant industry. Journal of Marketing Research, 34(1), 50-63.

Bertsekas, D. P. (2015). Dynamic programming and optimal control, volume 1. Athena Scientific Belmont, MA.

Bish, E. K. and Wang, Q. (2004). Optimal investment strategies for flexible resources, considering pricing and correlated demands. Operations Research, 52(6), 954-964.

Black, F. and Scholes, M. (1973). The pricing of options and corporate liabilities. The Journal of Political Economy, 81(3), 637 - 654.

Bollen, N. P. (1998). Valuing options in regime-switching models. The Journal of Derivatives, 6(1), 38-49.

Bollen, N. P. (1999). Real options and product life cycle. Management Science, 45(5), 670 - 684 .

Bolton, R. N. (1989). The relationship between market characteristics and promotional price elasticities. Marketing Science, 8(2), 153-169.

Boyle, P. P. (1988). A lattice framework for option pricing with two state variables. Journal of Financial and Quantitative Analysis, 23(1), 1-12. 
Brandao, L. E. and Dyer, J. S. (2005). Decision analysis and real options: A discrete time approach to real option valuation. Annals of Operations Research, 135(1), 21-39.

Bulan, L. T. (2005). Real options, irreversible investment and firm uncertainty: new evidence from us firms. Review of Financial Economics, 14(3-4), 255-279.

Chapra, S. C. and Canale, R. P. (1998). Numerical methods for engineers, volume 2. Mcgrawhill New York.

Cooper, R. and Kleinschmidt, E. (1986). An investigation into the new product process: steps, deficiencies, and impact. Product Innovation Management, 2(2), 71-85.

Cox, J. C., Ross, S. A., Rubinstein, M., et al. (1979). Option pricing: A simplified approach. Journal of financial Economics, 7(3), 229-263.

Danaher, P. J., Hardie, B. G., and Putsis Jr, W. P. (2001). Marketing-mix variables and the diffusion of successive generations of a technological innovation. Journal of Marketing Research, 38(4), 501-514.

Datar, S., Jordan, C. C., Kekre, S., Rajiv, S., and Srinivasan, K. (1997). Advantages of timebased new product development in a fast-cycle industry. Journal of Marketing Research, 34(1), 36-49.

Ding, Q., Dong, L., and Kouvelis, P. (2007). On the integration of production and financial hedging decisions in global markets. Operations Research, 55(3), 470-489.

Dockner, E. and Jorgensen, S. (1988). Optimal advertising policies for diffusion models of new product innovation in monopolistic situations. Management Science, 34(1), 119-130.

Dolan, R. J. and Jeuland, A. P. (1981). Experience curves and dynamic demand models: Implications for optimal pricing strategies. The Journal of Marketing, 45(1), 52-62.

Eliashberg, J. and Jeuland, A. P. (1986). The impact of competitive entry in a developing market upon dynamic pricing strategies. Marketing Science, 5(1), 20-36. 
Elmaghraby, S. E. (2011). Production capacity: Its bases, functions and measurement. In Planning Production and Inventories in the Extended Enterprise, pages 119-166. Springer.

Ernst, H., Hoyer, W. D., and Rübsaamen, C. (2010). Sales, marketing, and research-anddevelopment cooperation across new product development stages: implications for success. Journal of Marketing, 74(5), 80-92.

Ettl, M., Lu, Y., and Yao (2012). A production-inventory model for a push-pull manufacturing system with capacity and service level constraints. Production and Operations Management, 21(4), 668-681.

Fairhurst, A., Gable, M., and Dickinson, R. (1996). Determining advertising budgets for service enterprises. Journal of Services Marketing, 10(6), 18-32.

Fisher, L. (December 24, 1991). Cray loses only order for product supercomputer delay drives off lone buyer. New York Times, pp: D3.

Gao, J. and Jiang, L. (2010). The method of real options to encourage the R \& D team. Journal of Service Science and Management, 3(02), 235.

Garvin, M. J. and Cheah, C. Y. (2004). Valuation techniques for infrastructure investment decisions. Construction Management and Economics, 22(4), 373-383.

Golder, P. N. and Tellis, G. J. (2004). Growing, growing, gone: Cascades, diffusion, and turning points in the product life cycle. Marketing Science, 23(2), 207-218.

Govindan, K., Soleimani, H., and Kannan, D. (2015). Reverse logistics and closed-loop supply chain: A comprehensive review to explore the future. European Journal of Operational Research, 240(3), 603-626.

Hendricks, K. B. and Singhal, V. R. (1997). Delays in new product introductions and the market value of the firm: The consequences of being late to the market. Management Science, 43(4), 422-436. 
Hodder, J. E. and Riggs, H. E. (1985). Pitfalls in evaluating risky projects. Harvard Business Review, 63(1), 128-135.

Horsky, D. (1990). A diffusion model incorporating product benefits, price, income and information. Marketing Science, 9(4), 342-365.

Hull, J. and White, A. (1990). Valuing derivative securities using the explicit finite difference method. Journal of Financial and Quantitative Analysis, 25(01), 87-100.

Hull, J. C. (2009). Options, futures, and other derivatives. Prentice Hall.

Jain, D. C. and Rao, R. C. (1990). Effect of price on the demand for durables: Modeling, estimation, and findings. Journal of Business 85 Economic Statistics, 8(2), 163-170.

Jiang, Z. and Jain, D. C. (2012a). A generalized Norton-Bass model for multigeneration diffusion. Management Sience, 58(10), 1887-1897.

Jiang, Z. and Jain, D. C. (2012b). Optimal marketing entry time for successive product/service generation. Social Science Research Network, 83(5), 1-43.

Jones, J. M. and Ritz, C. J. (1991). Incorporating distribution into new product diffusion models. International Journal of Research in Marketing, 8(2), 91-112.

Jun, D. B. and Park, Y. S. (1999). A choice-based diffusion model for multiple generations of products. Technological Forecasting and Social Change, 61(1), 45-58.

Kalish, S. (1983). Monopolist pricing with dynamic demand and production cost. Marketing Science, 2(2), 135-159.

Kalish, S. (1985). A new product adoption model with price, advertising, and uncertainty. Management Science, 31(12), 1569-1585.

Kalish, S. (1988). Pricing new products from birth to decline: An expository review. Issues in pricing, pages 119-144. 
Kalish, S. and Lilien, G. (1986). A market entry timing model for new technologies. Management Science, 32(2), $194-205$.

Kamakura, W. A. and Balasubramanian, S. K. (1988). Long-term view of the diffusion of durables a study of the role of price and adoption influence processes via tests of nested models. International Journal of Research in Marketing, 5(1), 1-13.

Kamrad, B. and Ord, K. (2006). Market risk and process uncertainty in production operations. Naval Research Logistics, 53(7), 627-640.

Kapur, P., Aggarwal, A. G., Garmabaki, A. H. S., and Singh, G. (2013). Modelling diffusion of successive generations of technology: a general framework. International Journal of Operational Research, 16(4), 465-484.

Kapur, P., Aggarwal, A. G., Garmabaki, A. H., and Tandon, A. (2015). Multi-generational innovation diffusion modelling: a two dimensional approach. International Journal of Applied Management Science, 7(1), 1-18.

Kellogg, D. and Charnes, J. M. (2000). Real-options valuation for a biotechnology company. Financial Analysts Journal, 56(3), 76-84.

Kim, D. Y., Ashuri, B., and Han, S. H. (2012). Financial valuation of investments in international construction markets: Real options approach for market entry decisions. Journal of Management in Engineering, 29(4), 355-368.

Kornish, L. J. (2001). Pricing for a durable-goods monopolist under rapid sequential innovation. Management Science, 47(11), 1552-1561.

Krishnamoorthy, A., Prasad, A., and Sethi, S. P. (2010). Optimal pricing and advertising in a durable-good duopoly. European Journal of Operational Research, 200(2), 486-497.

Krishnan, T. V., Bass, F. M., and Jain, D. C. (1999). Optimal pricing strategy for new products. Management Science, 45(12), 1650-1663. 
Lander, D. M. and Pinches, G. E. (1998). Challenges to the practical implementation of modeling and valuing real options. The Quarterly Review of Economics and Finance, 38(3), 537-567.

Lilien, G. and Yoon, E. (1990). The timing competitive market entry:an exploratory study of new industrial products. Management Science, 36(2), 19-31.

Mahajan, V. and Muller, E. (1979). Innovation diffusion and new product growth models in marketing. The Journal of Marketing, 43(4), 55-68.

Mahajan, V. and Muller, E. (1991). Pricing and diffusion of primary and contingent products. Technological Forecasting and Social Change, 39(3), 291-307.

Mahajan, V. and Muller, E. (1996). Timing, diffusion, and substitution of successive generations of technological innovations: The IBM mainframe case. Technological Forecasting and Social Change, 51(2), 109-132.

Mahajan, V. and Sharma, S. (1986). A simple algebraic estimation procedure for innovation diffusion models of new product acceptance. Technological Forecasting and Social Change, $\mathbf{3 0}(4), 331-345$.

Markoff, J. (September 21, 1991). IBM workstation is delayed until 1992. The New York Times, pp: 35.

Miller, N. and Pazgal, A. (2007). Advertising budgets in competitive environments. Quantitative Marketing and Economics, 5(2), 131-161.

Mirantes, A. G., Población, J., and Serna, G. (2015). Commodity derivative valuation under a factor model with time-varying market prices of risk. Review of Derivatives Research, 18(1), 75-93.

Moorthy, K. S. and Png, I. P. (1992). Market segmentation, cannibalization, and the timing of product introductions. Management Science, 38(3), 345-359. 
Mun, J. (2002). Real options analysis: Tools and techniques for valuing strategic investments and decisions, volume 137. John Wiley \& Sons.

Myers, S. C. (1977). Determinants of corporate borrowing. Journal of Financial Economics, 5(2), 147-175.

Niu, S. (2006). A piecewise-diffusion model of new-product demands. Operations Research, 54(4), 678-695.

Norton, J. and Bass, F. (1987). A diffuion model of adoption and substitution for successive generations of high-technology products. Management Science, 33(9), 1-19.

Norton, L. W. J. (1989). Optimal entry timing for a product line extension. Marketing Science, 8(1), 1-17.

Ohashi, H. (2005). How does cannibalization affect the timing of new product introductions? evidence from US video games. In EARIE Conference, Porto.

O'Rourke, M. (2012). Inside Apple: How America's most admired and secretive company really works. Risk Management, 59(2), 43-44.

Padmanabhan, V. and Bass, F. M. (1993). Optimal pricing of successive generations of product advances. International Journal of Research in Marketing, 10(2), 185-207.

Pindyck, R. S. (1988). Irreversible investment, capacity choice, and the value of the firm. The American Economic Review, 78(5), 969-985.

Prasad, A., Bronnenberg, B., and Mahajan, V. (2004). Product entry timing in dual distribution channels: The case of the movie industry. Review of Marketing Science, 2(1), $1-18$.

Qi, J., Ding, Y., and Chen, L. (2008). Complex dynamics of the generic and brand advertising strategies in duopoly. Chaos, Solitons and Fractals, 36(2), 354-358. 
Qin, R. and Nembhard, D. A. (2012). Demand modeling of stochastic product diffusion over the life cycle. International Journal of Production Economics, 137(2), 201-210.

Rigopoulos, G. (2014). Real options adoption in capital budgeting: A highlight of recent literature. Journal of Economics and Business Research, 20(2), 41-51.

Rigopoulos, G. (2015). Real options valuation frameworks and adoption issues. International Journal of Information, Business and Management, 7(4), 1-13.

Rink, D. R. and Swan, J. E. (1979). Product life cycle research: A literature review. Journal of Business Research, 7(3), 219-242.

Robinson, B. and Lakhani, C. (1975). Dynamic price models for new-product planning. Management Science, 21(10), 1113-1122.

Ross, S. A. (1978). A simple approach to the valuation of risky streams. Journal of Business, $51,453-475$.

Ross, S. A. (1995). Uses, abuses, and alternatives to the net-present-value rule. Financial Management, 24(3), 96-102.

Ross, S. M. (1999). An introduction to mathematical finance. Cambridge University Press.

Schmalensee, R. (1978). A model of advertising and product quality. The Journal of Political Economy, 86(3), 485-503.

Schwartz, E. S. and Moon, M. (2000). Rational pricing of internet companies. Financial Analysts Journal, 56(3), 62-75.

Sethi, S. P. (1977). Dynamic optimal control models in advertising: a survey. SIAM Review, $\mathbf{1 9}(4), 685-725$.

Sethi, S. P., Prasad, A., and He, X. (2008). Optimal advertising and pricing in a new-product adoption model. Journal of Optimization Theory and Applications, 139(2), 351-360. 
Shankar, V. and Krishnamurthi, L. (1996). Relating price sensitivity to retailer promotional variables and pricing policy: an empirical analysis. Journal of Retailing, 72(3), 249-272.

Siu, T. K. and Yang, H. (2009). Option pricing when the regime-switching risk is priced. Acta Mathematicae Applicatae Sinica, English Series, 25(3), 369-388.

Soleimani, A., Kapur, P., Singh Jyotish, N., and Sanger Ragini, K. (2012). The optimal time of new generation product in the market. Communications in Dependability and Quality Management, 15(1), 123-137.

Soleimani, F., Khamseh, A. A., and Naderi, B. (2016). Optimal decisions in a dual-channel supply chain under simultaneous demand and production cost disruptions. Annals of Operations Research, 1(243), 301-321.

Speece, M. W. and Maclachlan, D. L. (1995). Application of a multi-generation diffusion model to milk container technology. Technological Forecasting and Social Change, 49(3), $281-295$.

Tannous, G. F. (1996). Capital budgeting for volume flexible equipment. Decision Sciences, $27(2), 157-184$.

Thompson, G. L. and Teng, J.-T. (1984). Optimal pricing and advertising policies for new product oligopoly models. Marketing Science, 3(2), 148-168.

Tilson, V. and Zheng, X. (2014). Monopoly production and pricing of finitely durable goods with strategic consumers fluctuating willingness to pay. International Journal of Production Economics, 154(2), 217-232.

Triantis, A. J. and Hodder, J. E. (1990). Valuing flexibility as a complex option. The Journal of Finance, 45(2), 549-565.

Trigeorgis, L. (1993). Real options and interactions with financial flexibility. Financial Management, 22(3), 202-224. 
Trigeorgis, L. (2005). Making use of real options simple: An overview and applications in flexible, modular decision making. The Engineering Economist, 50(1), 25-53.

Wahab, M. I. M. (2006). A lattice approach to the valuation of multi-variate contingent claims with regime switching. Ph.D. thesis, The University of Toronto.

Waldman, P. (September 26, 1986). Hewlett-packard, citing software snag, will delay new computer six months. Wall Street Journal, pp: 2.

Weng, Y.-C. (2009). Research on the optimum price and advertising strategy of the monopoly manufacturers. Journal of Information and Optimization Sciences, 30(4), 743-755.

Weng, Y. C. and Cheng, S. H. (2008). A study on union behavior not solid advertising, and business slander- an analysis of optimal price, advertising and R \& D strategies for a monopolistic competition firm. Fair Trade Quarterly, 16(38), 57 - 94.

Woodward, D. G. (1997). Life cycle costing-theory, information acquisition and application. International Journal of Project Management, 15(6), 335-344.

Wren, B. M., Souder, W. E., and Berkowitz, D. (2000). Market orientation and new product development in global industrial firms. Industrial Marketing Management, 29(6), 601-611.

Xiong, W. and Zhang, X. (2016). The real option value of renegotiation in public-private partnerships. Journal of Construction Engineering and Management, 8(200), 91-113.

Xu, X. and Birge, J. R. (2006). Equity valuation, production, and financial planning: A stochastic programming approach. Naval Research Logistics, 53(7), 641-655.

Zachary, G. P. (May 26, 1993). Climbing the peak: Agony and ecstasy of 200 code writers beget windows NT. Wall Street Journal, pp: A1.

Zhao, H. (2000). Raising awareness and signaling quality to uninformed consumers: A priceadvertising model. Marketing Science, 19(4), 390-396.

[title=References] 